Um estudo da teoria das dimensões aplicado a sistemas dinâmicos

\author{
Alex Pereira da Silva
}



SERVIÇO DE PÓS-GRADUAÇÃO DO ICMC-USP

Data de Depósito:

Assinatura:

\title{
Um estudo da teoria das dimensões aplicado a sistemas dinâmicos ${ }^{1}$
}

\begin{abstract}
Alex Pereira da Silva
Orientador: Prof. Dr. Alexandre Nolasco de Carvalho

Dissertação apresentada ao Instituto de Ciências Matemáticas e de Computação - ICMC-USP, como parte dos requisitos para obtenção do título de Mestre em Ciências - Matemática. VERSÃO REVISADA
\end{abstract}

USP - São Carlos

Maio de 2015

\footnotetext{
${ }^{1}$ Este trabalho teve o apoio financeiro da FAPESP.
} 
Ficha catalográfica elaborada pela Biblioteca Prof. Achille Bassi e Seção Técnica de Informática, ICMC/USP, com os dados fornecidos pelo(a) autor(a)

P586e $\begin{aligned} & \text { Pereira da Silva, Alex } \\ & \text { Um estudo da teoria das dimensões aplicado a } \\ & \text { sistemas dinâmicos / Alex Pereira da Silva; } \\ & \text { orientador Alexandre Nolasco de Carvalho. -- São } \\ & \text { Carlos, } 2015 . \\ & 203 \text { p. } \\ & \text { Dissertação (Mestrado - Programa de Pós-Graduação } \\ & \text { em Matemática) -- Instituto de Ciências Matemáticas } \\ & \text { e de Computação, Universidade de São Paulo, 2015. } \\ & \text { 1. semigrupo. 2. projeção do atrator global. 3. } \\ & \text { dimensão de Hausdorff. 4. dimensão fractal. I. } \\ & \text { Nolasco de Carvalho, Alexandre, orient. II. Título. }\end{aligned}$


"Grande coisa é haver recebido do céu uma partícula da sabedoria, o dom de achar as relações das coisas, a faculdade de as comparar e o talento de concluir!"

Memórias Póstumas de Brás Cubas, Machado de Assis 



\section{Agradecimentos}

Agradeço...

... de imediato, a Deus, pela sucessão de dificuldades e de superações que venho tendo e, consequentemente, pelo aprendizado que elas me trazem.

... a meus apoiadores pais, Juceline e Delmiro, e a meu radiante irmão, Eric, que me fazem reconhecer como a simplicidade da vida nos faz feliz.

... a meus orientadores, Kátia Andréia, Alexandre Nolasco e Éder Rítis, por me inspirarem em meio a dúvidas; por se fazerem presentes nas minhas escolhas; por compartilharem comigo o conhecimento que os anos lhes ofereceram.

... a meus amigos, os de perto e os de longe, dos matemáticos e químicos aos biólogos e filósofos, pois somos uma combinação convexa daqueles que passam por nós, mas eles não vão sós nem nos deixam sós: deixam um pouco de si e levam um pouco de nós.

... ao apoio financeiro da FAPESP neste trabalho e no da graduação, o qual foi imprescindível para o caminho que trilhei da graduação para a vida empresarial e então de volta para a vida acadêmica; por isso, também a agradeço. 


\section{Resumo}

Este trabalho se propõe a estudar o comportamento assintótico dos sistemas dinâmicos autônomos respaldado na Teoria das Dimensões. Mais precisamente, vamos compreender de que maneira nos é útil limitar a dimensão fractal do atrator global de um semigrupo a fim de estudar a dinâmica em dimensão finita, sem que se perca informações sobre a dinâmica ao fazê-lo. Para tanto, o Teorema de Mañé tem um papel decisivo junto às propriedades da dimensão de Hausdorff e a da dimensão fractal; nos permitindo encontrar uma projeção cuja restrição ao atrator é injetora sobre um espaço de dimensão finita. Constatamos ainda que esta abordagem por projeções se aplica largamente a semigrupos originados de equações diferenciais em espaços de Banach de dimensão infinita.

Palavras-chave: semigrupo, projeção do atrator global, dimensão de Hausdorff, dimensão fractal. 


\section{Abstract}

In this work, we study the asymptotic behavior of autonomous dynamical systems supported on the Dimension Theory. More precisely, we understand how fractal dimension finiteness of the global attractor of a semigroup can be used to study the dynamics in finite dimension, without losing information on the dynamics in doing so. For this purpose, the Mañés Theorem plays a decisive role considering the Hausdorff dimension properties and the fractal dimension; thanks to which we managed to find a projection whose restriction to the attractor is an injective application over a finite dimensional space. Besides, we also acknowledge that this projections approach is largely applied to semigroups arrising from differential equations in infinite dimensional Banach spaces.

Keywords: semigroup, projection of global attractor, Hausdorff dimension, fractal dimension. 


\section{Sumário}

Prefácio 1

1 Resultados Preliminares 11

1.1 Noções Básicas . . . . . . . . . . . . . . . . . . . . . . . . . 11

1.2 Semigrupos e seus Atratores . . . . . . . . . . . . . . . . . . . . . 21

1.2.1 Semigrupos Gradientes . . . . . . . . . . . . . . . . . . . . . 38

2 Teoria das Dimensões $\quad 47$

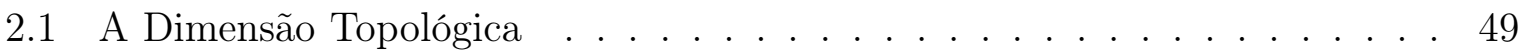

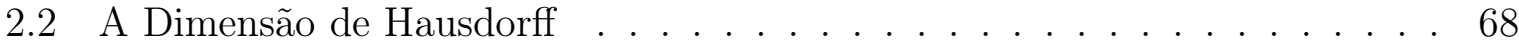

2.3 A Dimensão Fractal . . . . . . . . . . . . . . . . . . . . . . . 86

2.4 Um Comparativo . . . . . . . . . . . . . . . . . . . . . . 94

3 Redução a Dimensão Finita 101

3.1 O Teorema da Imersão . . . . . . . . . . . . . . . . . . . . . . . . . . 102

3.2 Projeções de Compactos e o Teorema de Mañé . . . . . . . . . . . . . . . . 108

4 Aplicacões $\quad 119$

4.1 Atratores de Semigrupos Gradientes . . . . . . . . . . . . . . . . . . . . . . 119

4.2 Atratores Exponenciais . . . . . . . . . . . . . . . . . . . . . . . . . . 128

4.3 Compactos Negativamente Invariantes . . . . . . . . . . . . . . . . . 137

5 Conclusões e Problemas em Aberto 153

A Espaços de Dimensão Topológica Nula 157

B Dimensões Indutivas $\quad 165$ 
C Integração de Funções Não Mensuráveis

D Medidas de Haar $\quad 185$

$\begin{array}{ll}\text { Índice Remissivo } & 199\end{array}$

$\begin{array}{ll}\text { Referências Bibliográficas } & 201\end{array}$ 


\section{Prefácio}

Compreender o mundo que nos cerca é um dos nossos desafios mais antigos e instigantes, senão o mais instigante. Desta busca, surgiu a modelagem matemática, que se faz usar muito frequentemente de equações diferenciais, as quais deram origem a uma importante sub-área da Matemática: a Teoria dos Sistemas Dinâmicos.

A fim de entender o que é um sistema dinâmico, imaginemos que queiramos prever o valor de uma dada variável $x$ em instantes futuros e denotemos o espaço dos seus possíveis valores por $X$, comumente chamado de espaço de fase. A variável $x$ pode descrever uma infinidade quantidades físicas, biológicas, econômicas, etc. E realmente queremos prever o comportamento de muitos acontecimentos e fenômenos observáveis, tais como o movimento de corpos celestes e a dinâmica de fluidos, que são de interesse da ciência já há séculos, são de fácil observação mas detêm complexos padrões de evolução.

Uma classe de tais problemas são de dimensão finita, no sentido de que finitos parâmetros são necessários para descrevê-lo. Podemos citar a posição de um corpo no espaço (um vetor em $\mathbb{R}^{3}$ ) ou a distribuição de temperaturas em um corpo $\Omega$ (uma função definida em $\Omega$ e tomando valores em $\mathbb{R}$ ); a população de uma certa bactéria (valores naturais); e o crescimento do PIB de um país ou a flutuação nos preços de uma dada ação (valores reais).

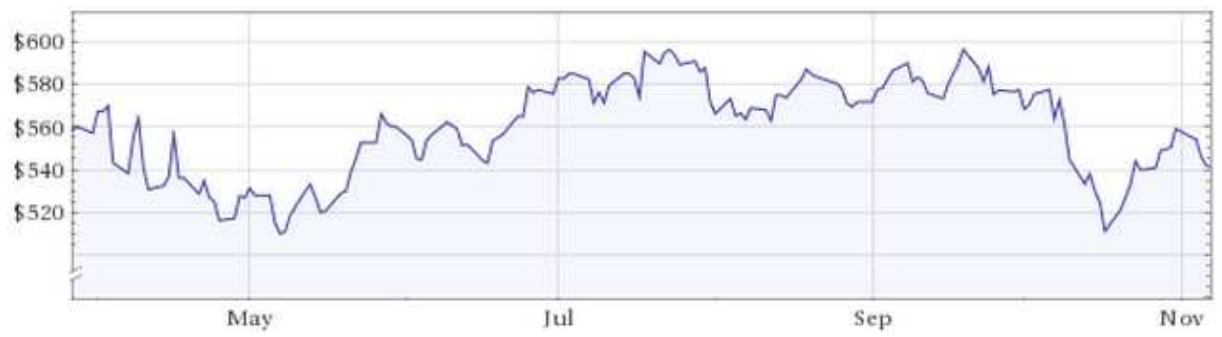

Figura 1: Preço da ação do Google. 
Outra classe são os de dimensão infinita, que surgiram muito mais recentemente devido aos avanços científico-tecnológicos, indo desde a problemas físicos com plasma e lasers até robótica e economia matemática.

Diferente dos sistemas lineares, os sistemas dinâmicos obedecem a leis muito mais complicadas, de modo que, em geral, a evolução de um estado inicial não pode ser prevista por meio de simples intuição ou cálculos elementares. O interesse então recai sobre estudar como os estados assintóticos do sistema se comportam sob perturbação, o que corresponde ao problema prático de determinar se estes estados assintóticos "permanecem" após tal perturbação; digamos como a economia de um país responde a uma pequena crise industrial no longo prazo.

Uma vez que um sistema dinâmico possui atrator global, todo seu comportamento assintótico pode ser descrito por meio de uma análise detalhada da dinâmica dentro deste conjunto. Isto acontece simplesmente porque o atrator global é definido para ser o objeto que detém todas as informações sobre a dinâmica assintótica: é o menor compacto para onde os pontos evoluem pela lei do sistema e de onde não saem.

Este trabalho se propõe a estudar a "complexidade" dos estados assintóticos dos sistemas dinâmicos respaldado na Teoria das Dimensões. Mais precisamente, vamos buscar compreender em que situações podemos estudar o atrator de um semigrupo em dimensão finita, sem que se perca informações sobre a dinâmica ao fazê-lo; e ainda constatar que esta abordagem se aplica largamente a problemas originados de equações diferenciais em espaços de Banach de dimensão infinita.

Em geral, o espaço de fase $X$ é um espaço métrico, denotado por $(X, \mathrm{~d})$, em que $\mathrm{d}(\cdot, \cdot): X \times X \rightarrow[0, \infty)$ indica a métrica de $X$. Quando for necessária a estrutura de espaço vetorial, tomaremos $X$ um espaço de Banach, de onde surge a necessidade de lidar com espaços de dimensão infinita.

Um sistema dinâmico (ou processo de evolução) é uma família de operadores

$$
S(t, s): X \rightarrow X
$$

a dois parâmetros, $s$ e $t$ reais, de forma que, dado o valor $x$ de uma variável no instante de tempo $s$, ao valor $S(t, s) x$ corresponde a evolução de $x$ para o instante futuro $t$. Em outras palavras, a família $\{S(t, s): t \geqslant s\}$ dita como um ponto $x \in X$ evolui ao longo do tempo pelo espaço de fase.

Algumas condições de compatibilidade devem ser impostas sobre tal família a fim de 
que o nome "processo de evolução" faça sentido. É de se esperar que a evolução de um ponto $x$ de um instante $t$ até o mesmo instante $t$ seja o próprio $x$, pois de fato não houve evolução. E também que, evoluir de um instante $t$ até um $\tau$ e então evoluir de $\tau$ até $s$ seja o mesmo que evoluir "diretamente" de $t$ até $s$. Por fim, vamos pedir que tal família seja contínua, visto que, em modelagem, dados e parâmetros são sempre aproximados e modelos que não gozem da propriedade de continuidade não têm muita utilidade.

Com isso, a família de operadores $\{S(t, s): t \geqslant s\}$ é um sistema dinâmico quando satisfaz

i) $S(t, t)=I_{X}$, para todo $t$ real;

ii) $S(t, \tau) S(\tau, s)=S(t, s)$, para todos os números reais $t \geqslant \tau \geqslant s$;

iii) a aplicação

$$
\{(t, s, x) \in \mathbb{R} \times \mathbb{R} \times X: t \geqslant s\} \ni(t, s, x) \longmapsto S(t, s) x \in X
$$

é contínua com respeito à topologia produto de $\mathbb{R} \times \mathbb{R} \times X$ e à topologia de $X$ induzida por d.

Tais sistemas aparecem frequentemente associados a equações diferenciais que podem ser ordinárias (EDO), parciais (EDP) ou funcionais (EDF).

Considere o problema

$$
\left\{\begin{array}{l}
x^{\prime}(t)=f(x) \\
x(0)=x_{0} \in \mathbb{R}^{m}
\end{array}\right.
$$

em que $f: \mathbb{R}^{m} \rightarrow \mathbb{R}^{m}$ é uma função continuamente diferenciável e tal que $f(x) \cdot x<0$, sempre que $|x| \geqslant M$, para algum $M>0$. O semigrupo associado à única solução da EDO (0.0.1) com $X=\mathbb{R}^{m}$ tem um atrator global.

Sejam $\Omega \subset \mathbb{R}^{3}$ é um aberto com fronteira $\Gamma$ e considere o seguinte problema: encontrar uma função $u(x, t) \in \bar{\Omega} \times[0, \infty) \rightarrow \mathbb{R}$ tal que

$$
\left\{\begin{array}{l}
\frac{\partial u}{\partial t}=\Delta u-u^{3} \text { em } \Omega \times(0, \infty) \\
u=0 \text { em } \Gamma \times(0, \infty) \\
u(x, 0)=u_{0}(x) \text { em } \Omega
\end{array}\right.
$$

em que $\Delta=\sum_{j=1}^{3} \frac{\partial^{2}}{\partial x_{i}^{2}}$ denota o operador Laplaciano nas variáveis espaciais $x=\left(x_{1}, x_{2}, x_{3}\right)$, o parâmetro $t$ é a variável tempo e $x \mapsto u_{0}(x)$ é uma função dada. A EDP (0.0.2) é dita 
uma equação do calor não linear e modela a distribuição de calor no domínio $\Omega$ no instante $t$ sujeita ao termo forçante não linear $u \mapsto-u^{3}$. Tal equação dá origem a um sistema dinâmico no espaço de fase $X=H^{1}(\Omega)$ que possui atrator global.

Por fim, considere a equação

$$
\left\{\begin{array}{l}
x^{\prime}(t)=x(t)(1-x(t-1)), t>0 \\
x(t)=\phi(t), \text { com }-1 \leqslant t \leqslant 0
\end{array}\right.
$$

é uma equação diferencial funcional com retardo, a qual origina um sistema dinâmico no espaço de fase $X=C\left([-1,0], \mathbb{R}_{+}\right)$, que também possui atrator global.

Apesar de já estar explícito, vale ressaltar que a evolução de um dado estado $x \in X$ pelo sistema dinâmico depende, a princípio, do instante inicial $s$ e do instante final $t$; ou equivalentemente, depende do instante inicial $s$ e do tempo transcorrido $t-s$. Quando, porém, a evolução não enxergar diferença entre distintos instantes iniciais mas apenas o tempo transcorrido, diremos que $\{S(t, s): t \geqslant s\}$ é um sistema dinâmico autônomo; isto é, quando

$$
S(t, s)=S(t-s, 0)
$$

para todos os tempos $t \geqslant s$. Neste caso, se definirmos $T(t)=S(t, 0)$, temos que

i) $T(0)=I_{X}$;

ii) $T(t) T(s)=T(t+s)$ para todo $t, s \in[0, \infty)$;

iii) $[0, \infty) \times X \ni(t, x) \mapsto T(t) x \in X$ é contínua.

Esta família $\{T(t): t \geqslant 0\}$ a um parâmetro real é dita um semigrupo e é o objeto de estudo deste texto. Note que, a partir de um semigrupo $\{T(t): t \geqslant 0\}$ podemos definir um sistema dinâmico autônomo $\{S(t, s): t \geqslant s\}$ fazendo $S(t, s)=T(t-s)$, para todos os tempos $t \geqslant s$. Assim, existe uma correspondência biunívoca entre semigrupos e sistemas dinâmicos autônomos, de modo que podemos intercambiar tais nomenclaturas.

Os demais sistemas dinâmicos serão chamados sistemas dinâmicos não autônomos, ou apenas, sistemas dinâmicos.

Os parâmetros envolvidos nas definições acima são todos reais, mas poderíamos considerar o caso em que são inteiros. No primeiro caso, dizemos que o sistema dinâmico é contínuo; já no segundo, que é discreto. O conceito de semigrupo vale da mesma maneira, bem como a ideia de evolução de uma variável num espaço de fases. A uma dada aplicação contínua $S: X \rightarrow X$ associamos o semigrupo $\left\{S^{n}: n=0,1,2 \ldots\right\}$ e, recipro- 
camente, a um dado semigrupo $\left\{T(1)^{n}: n=0,1,2 \ldots\right\}$ associamos a aplicação contínua $S=T(1): X \rightarrow X$.

Tendo como norte a compreensão dos atratores globais de semigrupos e sabendo que, para grande parte das equações que fornecem sistemas dinâmicos, trabalha-se com espaços vetoriais de dimensão infinita, investigar as boas propriedades do atrator $\mathcal{A} \subset X$ pode ser (em muitos casos) uma tarefa mais complexa se comparada com o caso $X=\mathbb{R}^{m}$. No entanto, recorrendo à teoria das dimensões, conseguimos projetar o atrator injetivamente em algum espaço euclidiano finito dimensional, espaços com os quais somos mais familiares e cujos objetos dinâmicos são supostamente mais simples. De certa forma, isto nos diz que podemos enxergar este objeto como tendo finitas direções e, ao fazê-lo, nada perdemos de informações topológicas nem da dinâmica do semigrupo.

Em segundo lugar, vemos condições para existência de atratores, procuramos entender como é o sistema dinâmico dentro do atrator, como os conjuntos limitados são atraídos para o atrator e como o atrator se comporta sob perturbação. Basicamente, a noção de atrator engloba noções precisas de atração e invariância pela ação de do semigrupo. Este objeto, de fato, contém todas as informações que buscamos para entender a evolução de um ponto $x$ pela ação do semigrupo.

A leitura deste trabalho pressupõe conhecimentos prévios em Teoria da Medida e Integração, Espaços Métricos e Análise Funcional, com os quais é possível desenvolver quase todo o conteúdo por vir, a menos de alguns tópicos adicionais, que serão postos nos apêndices. O Capítulo 1 estabelece a base sobre a qual vamos trabalhar: os resultados clássicos pressupostos e a teoria de semigrupos sobre a qual aplicaremos as noções de dimensão. Na Seção 1.1, fixamos algumas notações e listamos os resultados clássicos, destacando sua importância para os tópicos seguintes. Num segundo momento, abordamos a teoria de semigrupos, caracterizando os que possuem atrator global. Tratamos enfim dos semigrupos gradientes, um classe de semigrupos especialmente rica em informações sobre a dinâmica e que voltará a ser tratada no Capítulo 4, quando já teremos visto de que maneira a Teoria das Dimensões nos é útil. E isto encerra a Seção 1.2.

No Capítulo 2, tratamos em detalhes a Teoria das Dimensões com foco em três conceitos de dimensão: a topológica, a de Hausdorff e a fractal (largamente conhecida como upper box-counting dimension). O Teorema da Imersão 3.2 é o resultado que carrega a importância da dimensão topológica para os nossos propósitos, pois inspira o decisivo Teorema de Mañé 3.6, que aborda a dimensão de Hausdorff. Para efeitos práticos, porém, 
é com a dimensão fractal que mais trabalhamos pela relativa facilidade na obtenção de estimativas para a mesma. Isto nos ajuda a reconhecer o papel que cada uma das três tem.

Com a dimensão topológica, a qualquer espaço topológico (não vazio) associamos um número inteiro não negativo, o qual estende o que usualmente entendemos por dimensão e nos permite comparar espaços topológicos sob um ponto de vista antes não explorado. Sua definição, porém, traz dificuldades para o cômputo, mesmo quando se busca constatar que $\mathbb{R}^{m}$ tem dimensão topológica precisamente $m$. Simbolicamente, escrevemos $\operatorname{dim}_{\mathrm{T}}\left(\mathbb{R}^{m}\right)=m$. A Seção 2.1 basicamente se dedica a mostrar isto e, para tanto, recorre a uma noção equivalente de dimensão topológica em espaços métricos separáveis e se faz valer de resultados obtidos para espaços de dimensão topológica nula, os quais foram dispostos no Apêndice A para efeito de organização.

Mais restrita que a dimensão topológica, a dimensão de Hausdorff é definida para espaços métricos e é construída a partir de uma medida, a $\alpha$-medida de Hausdorff. É então uma abordagem via teoria da medida em vez de topológica e isto tem importantes implicações em termos de mensurar conjuntos de dimensão baixa em $\mathbb{R}^{m}$. Conjuntos que, do ponto de visto topológico, seriam zero dimensionais agora têm seu valor reconhecido, como por exemplo o conjunto de Cantor tradicional $C$ de $\mathbb{R}$, o qual tem medida de Lebesgue nula e também dimensão topológica nula. Porém, sua dimensão de Hausdorff é igual a $\alpha=\log 2 / \log 3$, de modo que a $\alpha$-medida de Hausdorff, para este valor de $\alpha$, enxerga a diferença entre um conjunto enumerável $E \subset \mathbb{R}$, o conjunto de Cantor $C \subset \mathbb{R}$ e o intervalo $[0,1] \subset \mathbb{R}$ associando a eles os valores 0,1 e $\infty$, respectivamente.

A Seção 2.2 se dedica a tornar precisas estas observações, apontar de que forma a $m$-medida de Hausdorff está relacionada à medida de Lebesgue de $\mathbb{R}^{m}$ e, a partir disso, verificar que $\mathbb{R}^{m}$ e os seus abertos têm dimensão de Hausdorff igual a $m$, com a notação $\operatorname{dim}_{\mathrm{H}}\left(\mathbb{R}^{m}\right)=m$. Além disso, a igualdade

$$
\operatorname{dim}\left(\mathbb{R}^{m}\right)=\operatorname{dim}_{\mathrm{H}}\left(\mathbb{R}^{m}\right)=\operatorname{dim}_{\mathrm{T}}\left(\mathbb{R}^{m}\right)=m
$$

estabelece um paralelo à noção usual de dimensão e nos diz que a dimensão de Hausdorff também preserva a intuição de dimensão dos espaços euclidianos. Entre muitas propriedades exploradas na Seção 2.2, uma de grande valor para o Teorema 3.6 de Mañé é a de que hiperfícies de $\mathbb{R}^{m}$ tem dimensão de Hausdorff $m-1$, o que concorda com a maneira como entendemos hiperfícies. 
Quando nos restringimos a espaços normados, não podemos esperar que, a desigualdade $\operatorname{dim}_{\mathrm{H}}\left(K_{1}+K_{2}\right) \leqslant \operatorname{dim}_{\mathrm{H}}\left(K_{1}\right)+\operatorname{dim}_{\mathrm{H}}\left(K_{2}\right)$ seja válida, com $K_{1}$ e $K_{2}$ compactos. No entanto, a dimensão fractal, $\operatorname{dim}_{\mathrm{F}}$, a satisfaz e felizmente domina a dimensão de Hausdorff. Na Seção 2.3, definimos a dimensão fractal para espaços métricos compactos, exploramos suas boas propriedades e destacamos de que forma a simplicidade da sua definição é vantajosa em relação à dimensão de Hausdorff. Exemplos de que as três noções de dimensão nem sempre coincidem são apresentados (de fato, raramente coincidem); um deles, inclusive, aponta para uma deficiência desta noção que não era visto nas outras duas: a dimensão fractal é sensível a conjuntos enumeráveis.

A Seção 2.4 encerra o Capítulo 2 estudando a maneira como estas três noções se relacionam em espaços métricos compactos $X$, que é

$$
\operatorname{dim}_{\mathrm{T}}(X) \leqslant \operatorname{dim}_{\mathrm{H}}(X) \leqslant \operatorname{dim}_{\mathrm{F}}(X)
$$

Para tanto, se faz valer de resultados profundos da Teoria das Dimensões, em particular do Teorema Fundamental da Teoria das Dimensões, o qual afirma que as noções de dimensão topológica, pequena dimensão indutiva e grande dimensão indutiva (livre tradução de small inductive dimension e large inductive dimension, respectivamente) coincidem em espaços métricos separáveis. Estas novas noções de dimensão são exploradas no Apêndice B, novamente com o intuito de manter o texto organizado e manter o foco da seção. Além disso, recorremos também ao Apêndice C, o qual contempla uma generalização da noção de integração usual de Lebesgue, se aplica a funções não mensuráveis e preserva importantes resultados da Teoria da Medida, tais como o Teorema da Convergência Monótona.

A partir da desigualdade (10.0.4), algumas propriedades de uma dimensão podem ser transferidas para outra; certamente vamos nos aproveitar deste fato no Capítulo 4 , quando tratarmos dos atratores globais dos semigrupos (os quais são, em particular, espaços métricos compactos).

Cabe ao Capítulo 3 lincar a Teoria das Dimensões à de Semigrupos por meio do Teorema de Mañé (Teorema [3.6), o qual garante, sob certas hipóteses sobre a dimensão de Hausdorff, que podemos projetar um subconjunto $\sigma$-compacto de um espaço de Banach injetivamente num subespaço de dimensão finita. A Seção 3.1 contempla o Teorema da Imersão (Teorema 3.2), que é uma aplicação do Teorema da Categoria de Baire (Teorema 1.14) e afirma que todo espaço topológico compacto de dimensão topológica finita pode 
ser imerso em $\mathbb{R}^{2 \operatorname{dim}_{\mathrm{T}}(X)+1}$. Vemos também que este profundo teorema é, de fato, o mais importante acerca da dimensão topológica e sua demonstração detém, com os devidos ajustes, a inspiração para o Teorema de Mañé, que é tratado em detalhes na Seção 3.2 .

Graças às propriedades da dimensão fractal e ao Teorema de Imersão, obtemos que atratores com dimensão fractal finita podem ser projetados injetivamente em algum $\mathbb{R}^{m}$, para $m$ suficientemente grande (Corolário 3.7). Sob a perspectiva prática, as hipóteses impostas são razoáveis, de modo que, para uma grande classe de sistemas dinâmicos autônomos, podemos enxergar o atrator em dimensão finita e ter a garantia de que não se perde informação ao fazê-lo, ainda que o atrator eventualmente seja um subconjunto de um espaço de dimensão infinita.

Construída a ponte entre as teorias dos Capítulos 1 e 2, o Capítulo 4 se encarrega de usá-la. Dividimos em três seções os tópicos de semigrupos gradientes, atratores exponenciais e compactos negativamente invariantes.

A Seção 4.1 aborda os semigrupos gradientes, que formam uma classe de semigrupos estáveis por perturbação e cuja dinâmica já é bem conhecida, no sentido de que a estrutura do atrator pode ser completamente descrita por conjuntos invariantes (limitados) isolados e as conexões entre eles. Estimamos a dimensão fractal do atrator por meio do máximo das dimensões de Hausdorff dos conjuntos instáveis locais de equilíbrios hiperbólicos.

Existem em abundância semigrupos que não são gradientes. Ainda assim, em geral, eles admitem objetos atratores tais que a "taxa de atração" é exponencial - os atratores exponenciais. A Seção 4.2 estuda como obter estes objetos a partir do fato de o atrator global do semigrupo ter dimensão fractal finita. Novamente, as condições impostas sobre os semigrupos são usualmente verificadas na prática, e isto nos diz que, em geral, atratores de semigrupos em espaços de Banach podem ser enxergados em espaços euclidianos finito dimensionais preservando a sua dinâmica original.

A Seção 4.3 trata de estimar a dimensão fractal de compactos negativamente invariantes, resultados de [23]. Para tanto, consideramos semigrupos admitem uma decomposição adequada como soma de um operador compacto de posto finito e de um operador "bem controlado".

O conteúdo se encerra com o Capítulo 5 em que, além de conclusões, fazemos considerações sobre que perguntas a comunidade matemática se propõe responder sobre projeções de atratores globais em espaços euclidianos.

Sob a proposta de fazer o texto ser autossuficiente, o máximo possível, os Apêndices 
A, B, C e D contemplam os importantes e curiosos conteúdos que foram necessários para desenvolver o Capítulo 2 de maneira organizada.

A Teoria das Dimensões detém seus maiores avanços quando trata de espaços métricos separáveis e, neste contexto, a dimensão topológica admite várias definições equivalentes (Teorema 2.17). Uma destas determina a dimensão topológica de maneira indutiva tendo em mente que $\operatorname{dim}_{\mathrm{T}}(\varnothing)=-1$. E mais, acontece que os espaços que têm dimensão topológica nula são os blocos que constroem espaços de dimensão topológica maior: todo espaço (métrico separável) que pode ser decomposto como reunião de até $m+1$ subespaços de dimensão topológica nula tem dimensão topológica no máximo $m$ (Teorema A.6); e reciprocamente (Corolário A.7). Este e outros resultados são encontrados no Apêndice A.

O Apêndice B se dedica a estudar as dimensões topológica, pequena indutiva e grande indutiva. Em espaços métricos separáveis, elas coincidem (Teorema B.11) e isto nos permite trabalhar com a que for mais conveniente. Na Seção 2.4, por exemplo, mostramos que a dimensão de Hausdorff domina a pequena dimensão indutiva, e só então concluímos que a dimensão de Hausdorff domina a dimensão topológica.

Grande parte dos cursos de Integração discutem a integração (de Lebesgue) de funções mensuráveis e alguns dos profundos resultados que esta teoria já alcançou. Pode ser surpreendente, porém, se dar conta de que não existem grandes dificuldades em estender esta teoria para funções não mensuráveis e ainda preservar resultados fundamentais como o Teorema da Convergência Monótona (Teorema C.5), Teorema de Fubini-Tonelli e Teorema de Lebesgue-Radon-Nikodym. Esta nova teoria é introduzida no Apêndice C a fim de justificar o que foi feito na Seção 2.4 .

Como $\mathbb{R}^{m}$ é um grupo topológico localmente compacto e Hausdorff, sabemos que ele admite uma única medida de Haar, no sentido de que, dadas duas medidas de Haar quaisquer em $\mathbb{R}^{m}$, elas são iguais, a menos de um ajuste por uma constante $c_{m}>0$. Acontece que tanto a medida de Lebesgue de $\mathbb{R}^{m}$ quanto a $m$-medida de Hausdorff em $\mathbb{R}^{m}$ são medidas de Haar. Esta relação fornece não só intuição às $\alpha$-medidas de Hausdorff como também nos diz que elas generalizam a medida de Lebesgue de tal forma que subconjuntos de $\mathbb{R}^{m}$ de dimensão mais baixa podem ser apropriadamente medidos, basta usar o valor de $\alpha$ correspondente a sua dimensão. Esses assuntos são de responsabilidade do Apêndice D e complementam o conteúdo da Seção 2.2,

A maior parte do resultados que discutimos ao longo deste texto serão demonstrados em detalhes, na intenção de fixar conceitos e compreender a típica argumentação tanto na Teoria de Semigrupos quanto na Teoria das Dimensões. Outros poucos serão apenas 
enunciados, mas com as devidas referências indicadas, isto porque sua demonstração foge ao intento deste trabalho, ou ainda porque são resultados tradicionais de cursos de Espaços Métricos, Teoria da Medida ou Análise Funcional, os quais são tomados como os prérequisitos para a leitura do presente texto. 


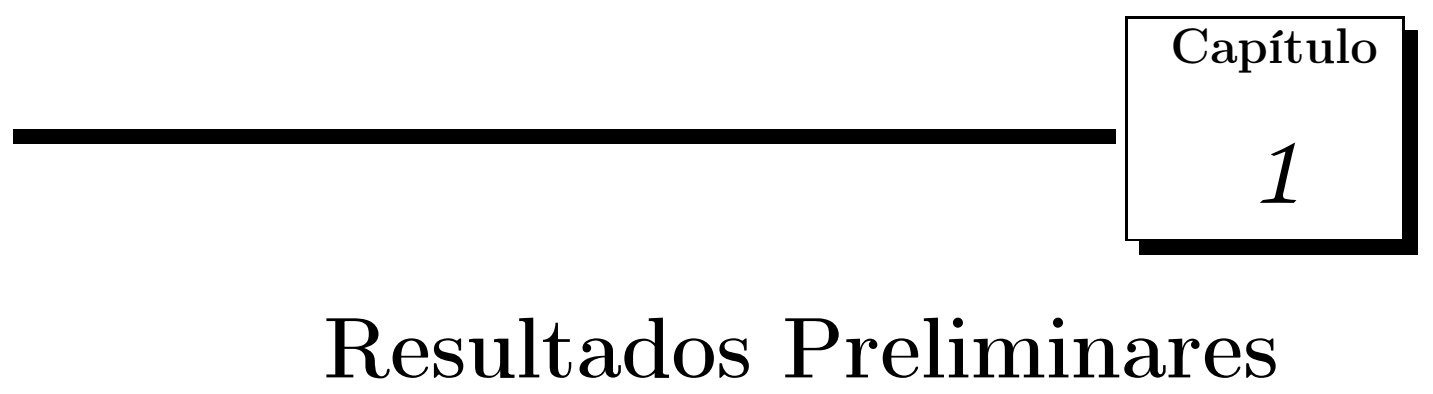

De início, os pré-requisitos da Teoria de Semigrupos não vão muito além de Espaços Métricos e resultados clássicos de Análise Funcional. Para um estudo completo, porém, são necessários alguns profundos tópicos de Análise Funcional, Distribuições, Espaços de Sobolev, Fórmula da Variação das Constantes, entre outros. Consulte [6].

A Seção 1.1lista os tópicos de Espaços Métricos, Teoria da Medida e Análise Funcional, dando referências e um direcionamento para o que se espera desses conteúdos.

Introduzimos na Seção 1.2 a Teoria de Semigrupos de maneira sucinta, buscando caracterizar semigrupos que têm atrator global e ainda apresentamos os semigrupos gradientes - classe de semigrupos de grande apelo prático na modelagem de fenômenos físicos.

A brevidade deste capítulo não faz com que a Teoria de Semigrupos seja um prérequisito, mas pressupõe que seja familiar ao leitor, para que os resultados do Capítulo 4 sejam completamente apreciados.

\subsection{Noções Básicas}

Assume-se familiaridade com os seguintes tópicos: espaços topológicos; continuidade; espaços métricos; separabilidade; compacidade; completude; espaços de Banach; topologias fraca e fraca*; construção de medidas de Borel em espaços métricos; medida de Lebesgue em $\mathbb{R}^{m}$; integração de Lebesgue; Teorema da Convergência Monótona; medidas produto e Teorema de Fubini-Tonelli.

Com isso, esta seção não se propõe a servir como um primeiro curso para tais assuntos, 
mas apenas relembrá-los e apontar as devidas referências para um tratamento completo; são elas: Bartle [2], Brezis [4], Folland [18], Hurewicz \& Wallman [20], Lima [22] e Munkres [25].

Indicaremos por $X=(X, \mathrm{~d})$ um espaço métrico com métrica d : $X \times X \mapsto[0, \infty)$ para todo o texto, a menos que dito contrário.

Um espaço topológico $X$ é dito compacto, quando toda cobertura aberta de $X$ admite uma subcobertura aberta finita.

Muitas soluções de importantes problemas matemáticos são obtidas como mínimos, máximos ou pontos fixos de funções em espaços normados de dimensão infinita, de modo que a compacidade então desempenha um papel de destaque nessas resoluções. Porém, espaços compactos nem sempre estão à disposição, especialmente quando trabalhamos com equações diferenciais. As duas principais alternativas a esse obstáculo são "enfraquecer" a topologia do espaço para que existam mais compactos ou enfraquecer a própria noção de compacidade na intenção de estender resultados a espaços mais gerais.

Em espaços métricos, podemos trabalhar com noções mais familiares de compacidade, conforme abaixo.

\section{Teorema 1.1. (Folland [18], Theorem 0.25)}

Dado $E \subset X$, são equivalentes:

a) E é completo e totalmente limitado;

b) (Propriedade de Bolzano-Weierstrass) Toda sequência em E admite uma subsequência que converge para um ponto de $\mathrm{E}$;

c) (Propriedade de Heine-Borel) Toda cobertura aberta de E admite uma subcobertura aberta finita.

Uma família $\mathcal{U}=\left\{U_{\alpha}\right\}_{\alpha \in \Lambda}$ de subconjuntos de um espaço topológico $X$ se diz localmente finita, quando todo ponto $x \in X$ admite uma vizinhança que intercepta apenas um quantidade finita de conjuntos $U_{\alpha}$.

Teorema 1.2. (Munkres [25], Lemma 39.1) Se $X$ é um espaço topológico e $\left\{F_{\alpha}\right\}_{\alpha \in \Lambda}$ é uma família localmente finita, então

$$
\overline{\bigcup_{\alpha \in \Lambda} F_{\alpha}}=\bigcup_{\alpha \in \Lambda} \overline{F_{\alpha}}
$$


Definição 1.3. Um espaço topológico $X$ é dito paracompacto, quando toda cobertura aberta de $X$ admite um refinamento aberto localmente finito que ainda cobre $X$.

Claro que espaços (topológicos) compactos são paracompactos.

Primordialmente, trabalhamos sobre espaços métricos separáveis, não somente porque as equações diferenciais usualmente se dão em espaços de Banach separáveis, mas também porque é sobre estes espaços que a Teoria das Dimensões mais avançou ao longo das décadas de pesquisa, desde o tradicional livro referência de Hurewicz \& Wallman [20] aos atuais expoentes da área.

\section{Teorema 1.4. (Lima [22], Proposição 9.1.1)}

São equivalentes:

a) X é separável, i.e., contém um subconjunto enumerável denso;

b) X possui uma base enumerável de abertos, i.e., existe uma coleção enumerável de abertos, $\mathcal{B}=\left\{B_{n}\right\}_{n \in \mathbb{N}}$, tais que todo aberto $U \subset X$ é escrito como alguma reunião de conjuntos $B_{n}$;

c) (Propriedade de Lindelöf) Toda cobertura aberta de X admite uma subcobertura aberta enumerável.

\section{Lema 1.5. (Lima [22], Proposição 9.5.9)}

Todo espaço métrico separável é paracompacto.

\section{Proposição 1.6. (Lima [22], Proposição 9.5.7)}

Em um espaço métrico separável, se $\left\{A_{\alpha}\right\}_{\alpha \in \Lambda}$ é uma família localmente finita, então $A_{\alpha}=\varnothing$, exceto para uma quantidade enumerável de indices $\alpha \in \Lambda$.

Sabemos (Munkres [25], Theorem 33.1) que a existência de uma função de Urysohn é garantida para espaços (topológicos) normais, i.e., aqueles em que conjuntos fechados disjuntos podem ser separados por abertos disjuntos. No entanto, já é suficiente considerar o caso particular em que o espaço é métrico.

\section{Lema 1.7. (Lema de Urysohn, Lima [22], Exemplo 6.7)}

Se $F_{1}$ e $F_{2}$ são subconjuntos de $X$ fechados e disjuntos, então existe uma função contínua $f: X \rightarrow[0,1]$ tal que $\left.f\right|_{F_{1}} \equiv 0$ e $\left.f\right|_{F_{2}} \equiv 1$. 


\section{Corolário 1.8. (Munkres [25], Lemma 31.1 e Theorem 32.2)}

Se $F_{1}$ e $F_{2}$ são subconjuntos de $X$ fechados e disjuntos, então

a) existem subconjuntos $U_{1}$ e $U_{2}$ de $X$ abertos e disjuntos tais que

$$
F_{1} \subset U_{1}, F_{2} \subset U_{2} e \overline{U_{1}} \cap \overline{U_{2}}=\varnothing .
$$

b) existe uma função contínua $f: X \rightarrow[0,1]$ tal que $f^{-1}(\{0\})=F_{1}$ e $f^{-1}(\{1\})=F_{2}$.

Vamos nos referir à função $f$ do item $b$ ) acima como uma função de Urysohn para o par de fechados disjuntos $F_{1}$ e $F_{2}$.

Uma das importantes aplicações da paracompacidade é a existência de partições da unidade (Veja Lima [22], Proposição 9.5.11), mas vamos nos concentrar no caso em que $X$ é um espaço métrico compacto, por conta da simplicidade e da aplicabilidade imediata para os nossos propósitos. Lembrando que o suporte de uma aplicação $\phi \in C(X,[0,1])$, denotado por supp $\phi$, é definido por

$$
\operatorname{supp} \phi=\overline{\{x \in X: f(x) \neq 0\}} \text {. }
$$

Definição 1.9. Seja $\left\{U_{1}, \ldots, U_{n}\right\}$ uma cobertura aberta finita de $X$. Dizemos que uma família $\left\{\phi_{1}, \ldots, \phi_{n}\right\} \subset C(X,[0,1])$ é uma partição da unidade subordinada à cobertura $\left\{U_{1}, \ldots, U_{n}\right\}$ se

i) $\operatorname{supp} \phi_{j} \subset U_{j}$, para todo $j=1, \ldots, n$;

ii) $\sum_{j=1}^{n} \phi_{j}(x)=1$, para todo $x \in X$.

O Teorema 36.1 em Munkres [25] garante a existência de uma partição da unidade subordinada à cobertura $\left\{U_{1}, \ldots, U_{n}\right\}$ quando $X$ é um espaço topológico normal. Apesar de mais restritivo, o resultado 1.10 é suficiente para os nossos propósitos e sua demonstração é notavelmente mais simples, usando o Lema 1.7.

\section{Teorema 1.10. (Existência de Partições da Unidade)}

Se $X$ é um espaço métrico compacto e $\left\{U_{1}, \ldots, U_{n}\right\}$ é uma cobertura aberta finita de $X$, então existe uma partição da unidade subordinada à cobertura $\left\{U_{1}, \ldots, U_{n}\right\}$. 


\section{Demonstração:}

Podemos escolher coberturas abertas $\left\{V_{1}, \ldots, V_{n}\right\}$ e $\left\{W_{1}, \ldots, W_{n}\right\}$ de $X$ tais que

$$
W_{i} \subset \overline{W_{i}} \subset V_{i} \subset \overline{V_{i}} \subset U_{i},
$$

para todo $i=1, \ldots, n$.

De fato, basta considerar $V_{1}=\mathcal{O}_{\delta_{1}}\left(A_{1}\right)$, em que

$$
A_{1}=X \backslash \bigcup_{j>1} U_{j}
$$

e $\delta_{1}=(1 / 2) \mathrm{d}\left(A_{1}, X \backslash U_{1}\right)$; daí repetir a construção considerando a cobertura aberta finita $\left\{V_{1}, U_{2}, \ldots, U_{n}\right\}$ e, por indução, obter $\left\{V_{1}, \ldots, V_{n}\right\}$. Procedendo da mesma forma, obtemos $\left\{W_{1}, \ldots, W_{n}\right\}$.

Considere as funções contínuas $\psi_{j}: X \rightarrow[0,1]$ dadas por

$$
\psi_{j}(x)=\frac{\mathrm{d}\left(x, X \backslash V_{j}\right)}{\mathrm{d}\left(x, W_{j}\right)+\mathrm{d}\left(x, X \backslash V_{j}\right)}
$$

e note que $\operatorname{supp} \psi_{j} \subset \bar{V}_{j} \subset U_{j}$ e

$$
\Psi(x)=\sum_{j=1}^{n} \psi_{j}(x) \geqslant 1, \forall x \in X
$$

Sendo assim, $\left\{\phi_{1}, \ldots, \phi_{n}\right\}$ forma uma partição da unidade subordinada à cobertura aberta finita $\left\{U_{1}, \ldots, U_{n}\right\}$, em que

$$
\phi_{j}(x)=\frac{\psi_{j}(x)}{\Psi(x)},
$$

para cada $j=1, \ldots, n$

Um ponto fixo de uma aplicação $T: X \rightarrow X$ é um ponto $x \in X$ invariante por $T$, ou seja, um ponto tal que $T(x)=x$. Muito embora não sejam imprescindíveis, resultados que garantam a existência de pontos fixos são muito convenientes para equações diferenciais, pois nos poupam de repetir argumentos padrão e nos permite manter a concentração nos elementos essenciais de tais problemas. O método das aproximações sucessivas é um bom 
representante dessa conveniência, pois a partir dele podemos determinar a solução de equações diferenciais ordinárias como sendo limite uniforme de funções contínuas.

Surpreendentemente, é na Seção 2.1 que o Teorema 1.11 será invocado, quando formos verificar que a dimensão topológica de $\mathbb{R}^{m}$ é precisamente $m$.

Teorema 1.11. (Teorema do Ponto Fixo de Brouwer, Hurewicz \& Wallman [20], Theorem 4.1.C)

Toda função contínua $f:[0,1]^{m} \rightarrow[0,1]^{m}$ admite um ponto fixo $\bar{x}$ em $[0,1]^{m}$.

Compacidade e continuidade são conceitos que, quando juntos, promovem resultados muito úteis, como o Teorema de Weierstrass.

\section{Proposição 1.12. (Teorema de Weierstrass, Lima [22], Proposição 8.2.4)}

Se $X$ é um espaço métrico compacto e $f: X \rightarrow \mathbb{R}$ é uma função contínua, então existem $x_{1}, x_{2} \in X$ tais que

$$
f\left(x_{1}\right) \leqslant f(x) \leqslant f\left(x_{2}\right)
$$

para todo $x \in X$.

Dentro dos nossos propósitos, os espaços de Baire são imprescindíveis para o Teorema da Imersão (Teorema 3.2) e o Teorema de Mañé (Teorema 3.6) do Capítulo 3, consequentemente, imprescindíveis para este trabalho.

Espaços métricos completos e espaços de Hausdorff compactos são espaços de Baire, os quais, embora sejam definidos de uma maneira longe de intuitiva, são uma ferramenta bem sofisticada e útil para as áreas de Topologia e Análise. Apenas a título de exemplo, considere o Teorema de Cantor: a interseção enumerável de fechados encaixados decrescentes em um espaço métrico completo é não vazia.

Dado um espaço topológico $X$, um subconjunto $A \subset X$ é dito de primeira categoria em $X$ se está contido numa reunião enumerável de subconjuntos fechados de $X$ de interior vazio. Caso contrário, é dito de segunda categoria em $X$.

Definição 1.13. Um espaço espaço topológico $X$ é dito um espaço de Baire quando todo aberto não vazio de $X$ é um conjunto de segunda categoria. 
Claramente, $(X, \mathrm{~d})$ é de segunda categoria em si mesmo se, e somente se, em qualquer representação de $X$ como reunião enumerável de fechados, pelo menos um deles contém uma bola aberta.

\section{Teorema 1.14. (Teorema da Categoria de Baire, Munkres [25], Theorem 48.2)}

Se X é um espaço métrico completo, então:

a) a reunião enumerável de fechados de interior vazio tem interior vazio;

b) a intersecção enumerável de abertos densos é densa;

c) $X$ é de segunda categoria em si mesmo;

Demonstração: Como os ítens $a$ ) e b) são obviamente equivalentes, vamos mostrar apenas $b)$ e $c$ ).

Para o item $b$ ), seja $\left\{A_{n}\right\}_{n} \subset X$ uma coleção de abertos densos em $X$.

Mostrar que $A=\bigcap_{n=1}^{\infty} A_{n}$ é denso em $X$ é mostrar que se $\mathcal{O}$ é um aberto qualquer de $X$, então $\mathcal{O} \cap A \neq \varnothing$.

Dado $x_{0} \in \mathcal{O}$, seja $r_{0}>0$ tal que $B_{X}\left(x_{0}, r_{0}\right) \subset \mathcal{O}$ e escolha $x_{1} \in B_{X}\left(x_{0}, r_{0}\right) \cap A_{1}$ e $0<r_{1}<r_{0} / 2$ tais que $\overline{B_{X}\left(x_{1}, r_{1}\right)} \subset B_{X}\left(x_{0}, r_{0}\right) \cap A_{1}$.

Por indução, tendo escolhido $x_{n}$ e $r_{n}$, tome $x_{n+1}$ e $r_{n+1}$ tais que $0<r_{n+1}<r_{n} / 2$ e

$$
\overline{B_{X}\left(x_{n+1}, r_{n+1}\right)} \subset B_{X}\left(x_{n}, r_{n}\right) \cap A_{n+1} .
$$

Assim, $\left\{x_{n}\right\}_{n}$ é uma sequência de Cauchy em $X$, logo existe $x=\lim _{n \rightarrow \infty} x_{n}$ em $X$. Uma vez que $x_{n+p} \in B_{X}\left(x_{n}, r_{n}\right)$, para todo $p \in \mathbb{N}$, segue que $x \in \frac{n \rightarrow \infty}{B_{X}\left(x_{n}, r_{n}\right)} \subset A_{n} \cap \mathcal{O}$, para todo $n \in \mathbb{N}$. Logo, $x \in A \cap \mathcal{O}$, como queríamos.

Quanto ao item c), vamos proceder por absurdo: suponha que podemos escrever

$$
X=\bigcup_{j=1}^{\infty} F_{j}
$$

em que cada $F_{j} \subset X$ é fechado de interior vazio. Equivalentemente, $\varnothing=\bigcap_{j=1}^{\infty} F_{j}^{C}$, o que contraria o item b). 
A seguir, consideramos um conceito que se prova surpreendentemente útil na Seção 2.2 para mostrar que a 1-medida de Hausdorff em $\mathbb{R}$ coincide com a medida de Lebesgue em $\mathbb{R}$ : o número de Lebesgue.

\section{Lema 1.15. (Número de Lebesgue - Munkres [25], Lemma 27.5)}

Se $X$ é um espaço métrico compacto e $\mathcal{U}$ é uma cobertura aberta de $X$, então existe um número $\delta>0$ de modo que, para cada $B \subset X$ com $\operatorname{diam}(B)<\delta$, tem-se que $B \subset U$, para algum $U \in \mathcal{U}$.

O número $\delta$ é dito um número de Lebesgue associado a $\mathcal{U}$.

O Teorema de Riesz (Brezis [4], Theorem 6.5) estabelece que, em espaços normados, a bola fechada unitária é compacta se, e somente se, o espaço é finito dimensional. Ou seja, em dimensão infinita, nenhum subconjunto com interior não vazio é compacto na topologia induzida pela norma. Isto dificulta a análise dos compactos de um tal espaço e consequentemente dificulta a análise de inúmeros problemas matemáticos. Mas como já citado, uma abordagem que traz bons resultados é dotar o espaço infinito dimensional de uma topologia com uma menor quantidade de conjuntos abertos, de modo que existam mais compactos, os quais podem ser manejados para resolver tais problemas.

Sejam $\left(X,\|\cdot\|_{X}\right)$ um espaço de Banach e $\mathcal{F}$ a família dos funcionais lineares contínuos $f:\left(X,\|\cdot\|_{X}\right) \rightarrow(\mathbb{R},|\cdot|)$. De fato, $\mathcal{F}$ é um espaço de Banach (mesmo que $X$ não o seja), o qual é chamado espaço dual de $X$ e denotado por $\left(X^{*},\|\cdot\|_{X^{*}}\right)$, em que

$$
\|f\|_{X^{*}}=\sup _{\substack{\|x\|<1 \\ x \in X}}|f(x)|
$$

A topologia fraca $\sigma\left(X, X^{*}\right)$ em $X$ é a topologia $\tau$ mais fina posta em $X$ de forma que a aplicação

$$
(X, \tau) \ni x \longmapsto f(x) \in(\mathbb{R},|\cdot|)
$$

é contínua, para todo $f \in \mathcal{F}$.

Noutros contextos, em vez de enfraquecer a topologia de $X$, podemos enfraquecer a topologia de $X^{*}$. A topologia fraca* $\sigma\left(X^{*}, X\right)$ em $X^{*}$ é a topologia $\varsigma$ mais fina posta em $X^{*}$ de forma que a aplicação

$$
\left(X^{*}, \varsigma\right) \ni f \longmapsto f(x) \in(\mathbb{R},|\cdot|)
$$


é contínua, para todo $x \in X$.

Sabidamente, os espaços topológicos $\left(X, \sigma\left(X, X^{*}\right)\right)$ e $\left(X^{*}, \sigma\left(X^{*}, X\right)\right)$ são espaços de Hausdorff (Brezis [4], Proposition 3.3 e Proposition 3.11). Além disso, a convergência nestas topologias é equivalente à convergência em $\mathbb{R}$, no sentido descrito no lema abaixo.

\section{Lema 1.16. (Brezis [4], Proposition 3.13)}

Se $\left(X,\|\cdot\|_{X}\right)$ é um espaço de Banach e $\left(f_{n}\right)_{n \in \mathbb{N}}$ uma sequência em $X^{*}$, então são equivalentes:

a) a sequência $\left(f_{n}\right)_{n}$ converge para $f \in X^{*}$ na topologia fraca* $\sigma\left(X^{*}, X\right)$ e

b) a sequência $\left(f_{n}(x)\right)_{n \in \mathbb{N}}$ converge para $f(x)$ em $\mathbb{R}$, qualquer que seja $x \in X$.

\section{Teorema 1.17. (Teorema de Hahn-Banach, Brezis [4], Corollaries 1.2, 1.4, 1.8)}

Sejam $\left(X,\|\cdot\|_{X}\right)$ um espaço de Banach e $Y$ um subespaço vetorial de $X$, então

a) todo funcional $f \in Y^{*}$ pode ser estendido a um funcional contínuo $F \in X^{*}$ de modo que $\|F\|_{X^{*}}=\|f\|_{Y^{*}}$;

b) se $Y$ não é denso em $X$, então existe um funcional $f \in X^{*}$ não nulo tal que $\left.f\right|_{Y} \equiv 0$;

c) para todo $x \in X$, vale

$$
\|x\|_{X}=\sup _{f \in B_{X^{*}}(0,1)}|f(x)|
$$

O próximo resultado, devido a Banach, Alaoglu e a Bourbaki, exibe o sucesso dessa abordagem, pois conseguimos a compacidade da bola fechada unitária na topologia fraca*.

\section{Teorema 1.18. (Teorema de Banach-Alaoglu, Brezis [4], Theorem 3.16)}

Em um espaço de Banach $\left(X,\|\cdot\|_{X}\right)$, a bola fechada unitária do espaço dual,

$$
B_{X^{*}}[0,1]=\left\{f \in X^{*}:\|f\|_{X^{*}}=1\right\},
$$

é compacta na topologia fraca* $\sigma\left(X^{*}, X\right)$.

Quando se quer impor a um conjunto uma topologia, uma das maneiras mais usuais e práticas é definir a topologia em termos de uma métrica no conjunto. A Análise Funcional 
recorre a esse método cotidianamente e dele obtém resultados de gigantesca aplicabilidade na matemática.

Por outro lado, um dos problemas de fundamental importância em Topologia é determinar condições sob as quais um espaço topológico é metrizável, pois resultados para espaços métricos são muito mais abundantes e facilitam a investigação de continuidade, compacidade, conexidade ...

O Teorema 1.18 nos forneceu um espaço (topológico) compacto com o qual trabalhar. É natural se perguntar se/quando tal espaço é metrizável.

\section{Teorema 1.19. (Brezis [4], Theorem 3.28)}

Seja $\left(X,\|\cdot\|_{X}\right)$ um espaço de Banach.

Então $X$ é separável se, e somente se, $\left(B_{X^{*}}[0,1], \sigma\left(X^{*}, X\right)\right)$ é metrizável.

Os seguintes três resultados e seus pré-requisitos implícitos são basicamente o que usaremos da Teoria da Medida, sem mencionar os tópicos de integração de funções não mensuráveis que vamos expor no Apêndice C.

Para um curso completo, consulte Bartle [2] e Folland [18].

\section{Teorema 1.20. (Teorema de Carathéodory, Folland [18], Theorem 1.11)}

Se $\mu^{*}$ é uma medida exterior, então a coleção $\mathcal{M}$ dos conjuntos $\mu^{*}$-mensuráveis é uma $\sigma$-álgebra e $\left.\mu^{*}\right|_{\mathcal{M}}$ é uma medida completa.

\section{Teorema 1.21. (Teorema da Convergência Monótona, Folland [18], Theorem}

\subsection{4)}

Se $\left(f_{n}\right)_{n}$ é uma sequência de funções mensuráveis de $X$ em $\overline{\mathbb{R}}$ tal que $f_{n} \leqslant f_{n+1}$, então

$$
\int \lim _{n \rightarrow \infty} f_{n}=\lim _{n \rightarrow \infty} \int f_{n}
$$

Corolários envolvendo somas (finitas ou enumeráveis) estão implicitamente assumidos.

$\operatorname{Sejam~}(X, \mathcal{M}, \mu)$ e $(Y, \mathcal{N}, \nu)$ espaços de medida e considere uma função $f: X \times Y \rightarrow \bar{R}$. Definimos a $x$-seção de $f$, denotando por $f_{x}$, e a $y$-seção de $f$, denotando por $f^{y}$, como sendo

$$
f_{x}(y)=f^{y}(x)=f(x, y)
$$


Teorema 1.22. (Teorema de Fubini-Tonelli, Folland [18], Theorem 2.37)

$\operatorname{Sejam}(X, \mathcal{M}, \mu)$ e $(Y, \mathcal{N}, \nu)$ espaços de medida $\sigma$-finitos, e uma função $f: X \times Y \rightarrow \overline{\mathbb{R}}$. Valem as seguintes afirmações:

1) (Tonelli) se $f$ é mensurável não negativa, então as funções

$$
g(x)=\int f_{x} d \nu \quad e h(y)=\int f^{y} d \mu
$$

são mensuráveis não negativas de $X$ e de $Y$, respectivamente, e vale

$$
\int f d(\mu \times \nu)=\int\left(\int f(x, y) d \nu(y)\right) d \mu(x)=\int\left(\int f(x, y) d \mu(x)\right) d \nu(y) .
$$

2) (Fubini) se $f$ é integrável com respeito a $\mu \times \nu$, então

i) $f_{x}$ é integrável com respeito a $\nu$, para quase todo $x \in X$;

ii) $f^{y}$ é integrável com respeito a $\mu$, para quase todo $y \in Y$;

iii) $g(x)$ está definida para quase todo ponto e é integrável com respeito a $\mu$;

iv) $h(y)$ está definida para quase todo ponto e é integrável com respeito a $\nu$;

v) vale (1.1.1).

\subsection{Semigrupos e seus Atratores}

Os atratores globais detêm o papel central no estudo dos semigrupos. Vamos investigar que propriedades um semigrupo demanda para ter um atrator global, caracterizandoos completamente por meio de compacidade assintótica e dissipatividade. Para tanto, conceitos de "atração" e "invariância" precisam ser bem delimitados, assim como o conceito de $\omega$-limite, o qual carrega a essência do comportamento assintótico do semigrupo e pode ser entendido como a região na qual órbitas se acumulam.

Em termos práticos, a caracterização envolve propriedades mais difíceis de serem verificadas. Por isso, apresentamos também resultados que garantam a existência do atrator sob condições mais acessíveis. Com isso, surgem os conceitos de semigrupo eventualmente compacto, eventualmente limitado e ponto dissipativo. Citamos o Teorema 1.39 
como um exemplar de que condições ainda menos restritivas podem ser impostas; sua demonstração, porém, vai além do que nos propomos.

Quando se procura entender a estrutura de um atrator, a classe dos semigrupos gradientes é certamente uma das mais generosas em informações: podemos determinar a dinâmica do semigrupo por meio dos seus invariantes isolados ao expressar o atrator global em termos deles. Além disso, tais semigrupos não admitem estruturas homoclínicas e, de fato, o conceito de par atrator-repulsor pode ser usado para entender que a estrutura globalmente.

As referências [1], [6], [8] e [28] fornecem um tratamento mais completo da teoria que introduzimos aqui.

Sejam $X$ um espaço métrico e d : $X \times X \rightarrow[0, \infty)$ sua métrica. Denote por $\mathcal{C}(X)$ o conjunto das aplicações contínuas de $X$ em $X$.

Definição 1.23. Um semigrupo (ou sistema dinâmico autônomo) em $X$ é uma família a um parâmetro $t$, real não negativo, de aplicações contínuas de $X$ em $X$, denotada por $\{T(t): t \geqslant 0\} \subset \mathcal{C}(X)$, que satisfaz três propriedades:

(i) $T(0)=I_{X}$;

(ii) $T(t+s)=T(t) T(s)$, para todos os $t, s \geqslant 0$; (propriedade de semigrupo)

(iii) a aplicação $[0, \infty) \times X \ni(t, x) \longmapsto T(t) x \in X$ é contínua, pondo em $X$ a topologia induzida por d e em $[0, \infty) \times X$ a topologia produto. (propriedade de continuidade do semigrupo)

Mantendo a correspondência com os processos de evolução, as condições (i) e (ii) são ditas condições de compatibilidade.

Segue de (ii) que evoluir de um instante 0 até um instante $s$ e então evoluir de $s$ até $t$ é o mesmo que evoluir "diretamente" de 0 até $s+t$; além disso, as aplicações $T(\cdot)$ comutam:

$$
T(t) T(s)=T(t+s)=T(s+t)=T(s) T(t)
$$

para todos os instantes $t, s \geqslant 0$.

Note que $(\{T(t): t \geqslant 0\}, \circ)$ é de fato um semigrupo (comutativo) com a operação de composição de aplicações. E não é hipótese, em momento algum, que tais aplicações sejam 
homeomorfismos. Mas se este fosse o caso, obteríamos uma nova família, $\{T(t): t \in \mathbb{R}\}$, definindo $T(t):=T(-t)^{-1}$, para todo $t<0$, a qual é um grupo (comutativo) com a operação de composição.

Vamos usar a notação $T(\cdot)$ para nos referirmos ao semigrupo $\{T(t): t \geqslant 0\}$.

Agora vamos definir o objeto dinâmico que contém todos os estados assintóticos de um semigrupo, o atrator global do semigrupo. Além de compacto, este objeto é para onde todos os pontos evoluem pela ação do semigrupo e de onde não saem. Estas expressões se referem às noções de atração e invariância pela ação do semigrupo $T(\cdot)$.

Definição 1.24. Dizemos que um conjunto $A \subset X$ é invariante, ou mais precisamente, invariante pela ação $T(\cdot)$, quando, para todo $t \geqslant 0$,

$$
T(t) A=A,
$$

que é o mesmo que dizer que todo ponto de A é a evolução por $T(t)$ de algum outro ponto de $A$ e também que todo ponto de $A$, ao evoluir por $T(t)$, permanece em $A$, para cada $t \geqslant 0$.

É comum dizer que um conjunto $A$ invariante tem dinâmica própria, pois, como vamos mostrar, ele é uma reunião de órbitas que não saem de $A$. Essa noção será melhor entendida quando definirmos o que são órbitas e conjunto atrator.

Note que, dada uma família de conjuntos invariantes $\left\{A_{\lambda}\right\}_{\lambda \in \Lambda} \subset X$, sua reunião é também invariante: dado $t \geqslant 0$, temos $T(t) A_{\lambda}=A_{\lambda}$ e $T(t)\left(\bigcup_{\lambda \in \Lambda} A_{\lambda}\right)=\bigcup_{\lambda \in \Lambda} T(t) A_{\lambda}$.

Uma solução global de $T(\cdot)$ é uma aplicação $\xi: \mathbb{R} \rightarrow X$ tal que, para todos os $t \geqslant 0$ e $\tau \in \mathbb{R}$, tem-se

$$
T(t) \xi(\tau)=\xi(t+\tau)
$$

Neste caso, a sua imagem $\{\xi(\tau): \tau \in \mathbb{R}\} \subset X$ é denotada por $\gamma(\xi)$ e é chamada de a órbita de $\xi$, ou mais precisamente, a órbita global da solução $\xi$.

Lema 1.25. Toda solução global é uma aplicação contínua.

Demonstração: Dados $t_{0} \in \mathbb{R}$ e $\varepsilon>0$, seja $\tau<t_{0}-\varepsilon$. 
Para qualquer $t \in\left(t_{0}-\varepsilon, t_{0}+\varepsilon\right)$, podemos escrever

$$
d\left(\xi(t), \xi\left(t_{0}\right)\right)=d\left(T(t-\tau) \xi(\tau), T\left(t_{0}-\tau\right) \xi(\tau)\right)
$$

de modo que a continuidade do semigrupo $T(\cdot)$ implica a continuidade de $\xi$.

Dizemos que uma solução global $\xi$ constante igual a $z^{*}$ é um ponto de equilíbrio ou solução de equilíbrio ou solução estacionária. Neste caso, o conjunto unitário $\left\{z^{*}\right\}$ é invariante: $z^{*}=\xi(t+\tau)=T(t) \xi(\tau)=T(t) z^{*}$, para todo $t \geqslant 0$.

Uma solução periódica é uma solução global $\xi$ para a qual existe $\sigma>0$ tal que $\xi(t+\sigma)=\xi(t)$, para todo $t$ real. Neste caso, $\gamma(\xi)$ é dita uma órbita periódica.

É de se esperar que um atrator global seja composto por tais objetos dinâmicos elementares.

Lema 1.26. Toda órbita global é um conjunto invariante.

Demonstração: Sejam $\xi: \mathbb{R} \longrightarrow X$ uma solução global e $\gamma(\xi)$ a sua órbita.

Dados $z \in \gamma(\xi)$ e $t \geqslant 0$, temos $z=\xi(\tau)$, para algum $\tau \in \mathbb{R}$.

Por um lado, $T(t) z=T(t) \xi(\tau)=\xi(t+\tau) \in \gamma(\xi)$, daí $T(t)(\gamma(\xi)) \subset \gamma(\xi)$. Por outro,

$$
z=\xi(\tau)=\xi(t+(\tau-t))=T(t) \xi(\tau-t)
$$

de modo que $\gamma(\xi) \subset T(t)(\gamma(\xi))$.

Teorema 1.27. Um conjunto $A \subset X$ é invariante se, e somente se, é reunião de órbitas globais.

Demonstração: Suponha que $A$ seja invariante e tome $x_{0} \in A$.

Por definição de invariância, existe $x_{-1} \in A$ tal que $T(1) x_{-1}=x_{0}$. Novamente, existe $x_{-2} \in A$ tal que $T(1) x_{-2}=x_{-1}$, e por indução, obtemos $\left(x_{-n}\right)_{n \in \mathbb{N}} \subset A$ tal que

$$
T(1) x_{-n}=x_{-n+1}, \forall n \in \mathbb{N} \text {. }
$$

Note que $T(2) x_{-2}=T(1) T(1) x_{-2}=T(1) x_{-1}=x_{0}$ e que também por indução tem-se $T(n) x_{-m}=x_{n-m}, \forall n, m \in \mathbb{N} \operatorname{com} n \leqslant m$. 
Definimos então $\xi: \mathbb{R} \longrightarrow X$ por

$$
\xi(\tau)= \begin{cases}T(\tau) x_{0} & \text { se } \tau \geqslant 0 \\ T(\tau+n) x_{-n} & \text { se }-n \leqslant \tau<-n+1\end{cases}
$$

de modo que $\xi$ está bem definida em $\mathbb{R}$ e $\xi(\tau) \in A, \forall \tau \in \mathbb{R}$, pois $A$ é invariante.

Mostremos que $\xi$ é solução global de $T(\cdot)$. Sejam $t \geqslant 0$ e $\tau \in \mathbb{R}$, daí temos alguns casos a considerar:

Se $\tau \geqslant 0$, então

$$
T(t) \xi(\tau)=T(t) T(\tau) x_{0}=T(t+\tau) x_{0}=\xi(t+\tau) .
$$

Agora se $\tau<0$ é tal que $t+\tau \geqslant 0$, tome $n$ tal que $-n \leqslant \tau<-n+1$, daí

$$
T(t) \xi(\tau)=T(t) T(\tau+n) x_{-n}=T(t+\tau) T(n) x_{-n}=T(t+\tau) x_{0},
$$

de modo que $T(t) \xi(\tau)=\xi(t+\tau)$.

E por fim, se $\tau<0$ é tal que $t+\tau<0$, tome $n, m$ tais que $-n \leqslant \tau<-n+1$ e $-m \leqslant t+\tau<-m+1$, daí $m \leqslant n$ e

$$
\begin{aligned}
T(t) \xi(\tau) & =T(t) T(\tau+n) x_{-n} \\
& =T((t+\tau+m)+(n-m)) x_{-n} \\
& =T(t+\tau+m) T(n-m) x_{-n} \\
& =T(t+\tau+m) x_{n-m-n} \\
& =\xi(t+\tau) .
\end{aligned}
$$

Pelo exposto, obtemos que por todo ponto de $A$ passa uma órbita global inteiramente contida em $A$ e portanto $A$ é reunião dessas órbitas.

Reciprocamente, se $A$ é reunião de órbitas globais, as quais são conjuntos invariantes, então como observamos isto implica que $A$ é também invariante.

Ficou implícita na demonstração acima a convenção de que o conjunto dos números naturais, $\mathbb{N}$, é dado por $\{1,2, \ldots\}$. Quando for necessário incluir o elemento 0 , usaremos 
os inteiros não negativos: $\mathbb{Z}_{+}=\{0,1,2, \ldots\}$.

Considere conjuntos $A, B \subset X$ como na figura abaixo:

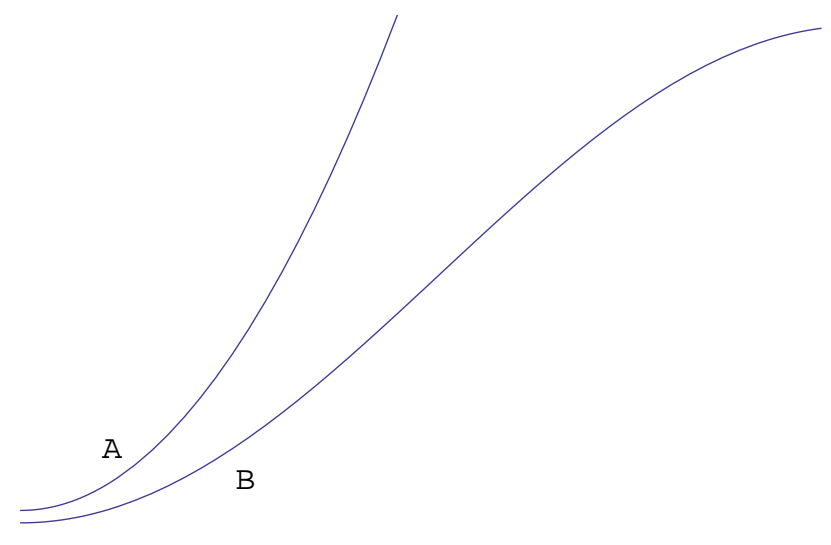

Note que usando a métrica de $X$, obtemos $d(A, B)$ bem "pequeno", mas que esses conjuntos, se vistos por exemplo como as soluções de uma EDO e desta mesma EDO perturbada, são bastante distintos. Para efeito de mensurar quão próximos são objetos relacionados à dinâmica de um sistema, a métrica d não é conveniente. E como o comportamento assintótico dos sistemas dinâmicos é um dos pontos chave desta teoria, precisamos de uma noção de "distância" que considere essas discrepâncias, mais precisamente, uma que indique que $A$ está $\varepsilon$ próximo de $B$ quando $A$ estiver na $\varepsilon$-vizinhança de $B$. Vale lembrar que, dado $\varepsilon>0$, a $\varepsilon$-vizinhança de $B$, indicada por $\mathcal{O}_{\varepsilon}(B)$, é o conjunto definido por

$$
\mathcal{O}_{\varepsilon}(B)=\bigcup_{b \in B} B_{X}(b, \varepsilon)=\{x \in X: \mathrm{d}(x, B)<\varepsilon\} .
$$

Dados $A, B \subset X$ não vazios, definimos a semidistância de Hausdorff de $A$ até $B$, indicada por $\operatorname{dist}_{\mathrm{H}}(A, B)$, como sendo

$$
\operatorname{dist}_{\mathrm{H}}(A, B)=\sup _{a \in A} \inf _{b \in B} \mathrm{~d}(a, b)=\sup _{a \in A} \mathrm{~d}(a, B),
$$

e que naturalmente $\mathrm{d}(a, B)$ indica, para cada $a \in A$, o ínfimo das distâncias $\mathrm{d}(a, b)$ para $b \in B$.

Devemos enfatizar que $\operatorname{dist}_{\mathrm{H}}(A, B)$ é chamada a semidistância de Hausdorff de $A$ até $B$, pois $\operatorname{dist}_{\mathrm{H}}$ não é simétrica, i.e., não é verdade que $\operatorname{dist}_{\mathrm{H}}(A, B)=\operatorname{dist}_{\mathrm{H}}(B, A)$, para todos os $A, B \subset X$. Note, porém, que dist $_{\mathrm{H}}$ respeita a desigualdade triangular: dados 
$a, b, c$ em $A, B, C$, respectivamente,

$$
\begin{aligned}
\mathrm{d}(a, b) \leqslant \mathrm{d}(a, c)+\mathrm{d}(c, b) & \Rightarrow \inf _{b \in B} \mathrm{~d}(a, b) \leqslant \mathrm{d}(a, c)+\inf _{b \in B} \mathrm{~d}(c, b) \\
& \Rightarrow \mathrm{d}(a, B) \leqslant \inf _{c \in C} \mathrm{~d}(a, c)+\sup _{c \in C} \mathrm{~d}(c, B)
\end{aligned}
$$

e então basta passar o supremo com $a \in A$ para obter

$$
\operatorname{dist}_{\mathrm{H}}(A, B) \leqslant \operatorname{dist}_{\mathrm{H}}(A, C)+\operatorname{dist}_{\mathrm{H}}(C, B) .
$$

Não é difícil verificar que $A \subset \bar{B}$ se, e somente se, $\operatorname{dist}_{\mathrm{H}}(A, B)=0$.

De fato, se $A \subset \bar{B}$, então todo ponto de $A$ é limite de pontos de $B$, logo para todo $a \in A$ vale $0=\mathrm{d}(a, B)$. Por outro lado, se $\sup _{a \in A} \mathrm{~d}(a, B)=0$, então $\mathrm{d}(a, B)=0$ para todo $a \in A$, i.e., $a \in \bar{B}, \forall a \in A$.

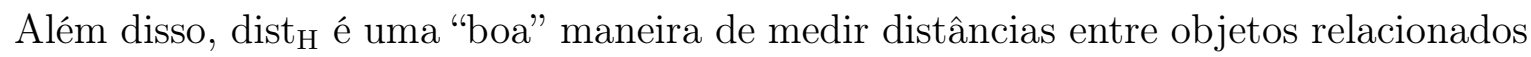
à dinâmica assintótica do semigrupo, pois sob esta noção, $A$ está $\varepsilon$-próximo de $B$ se, e somente se, $A$ está na $\varepsilon$-vizinhança de $B$. De fato, se $\operatorname{dist}_{\mathrm{H}}(A, B)<\varepsilon$, então $\mathrm{d}(a, B)<$ $\varepsilon, \forall a \in A$, que por definição de $\varepsilon$-vizinhança implica $A \subset \mathcal{O}_{\varepsilon}(B)$. Agora, se $A \subset \mathcal{O}_{\varepsilon}(B)$, então $\mathrm{d}(a, B) \leqslant \varepsilon, \forall a \in A$, consequentemente $\operatorname{dist}_{\mathrm{H}}(A, B) \leqslant \varepsilon$.

A distância simétrica de Hausdorff entre $A$ e $B$, indicada por $\mathrm{d}_{H}(A, B)$, é definida por

$$
\mathrm{d}_{\mathrm{H}}(A, B)=\operatorname{dist}_{\mathrm{H}}(A, B)+\operatorname{dist}_{\mathrm{H}}(B, A) .
$$

Com isso, $\mathrm{d}_{\mathrm{H}}: \mathcal{F}_{B} \times \mathcal{F}_{B} \rightarrow \mathbb{R}$ é uma métrica, em que $\mathcal{F}_{B}$ denota a coleção dos fechados limitados não vazios de $X$, pois

$$
\mathrm{d}_{\mathrm{H}}(A, B)=0 \Leftrightarrow\left\{\begin{array} { l } 
{ \operatorname { d i s t } _ { \mathrm { H } } ( A , B ) = 0 } \\
{ \operatorname { d i s t } _ { \mathrm { H } } ( B , A ) = 0 }
\end{array} \Leftrightarrow \left\{\begin{array}{l}
A \subset \bar{B}=B \\
B \subset \bar{A}=A
\end{array} \Leftrightarrow A=B .\right.\right.
$$

Definição 1.28. Dados $A, B \subset X$, dizemos que $A$ atrai $B$ pela ação de $T(\cdot)$, ou que $B$ é atraído por A pela ação de $T(\cdot)$, quando

$$
\lim _{t \rightarrow \infty} \operatorname{dist}_{\mathrm{H}}(T(t) B, A)=0 .
$$

Note que $A$ atrai $B$ se, e somente se, para todo $\varepsilon>0$, existe $\tau=\tau(\varepsilon, A, B)>0$ tal que $T(t) B \subset \mathcal{O}_{\varepsilon}(A)$, para todo $t \geqslant \tau$. 
Agora, sabemos precisamente o que quer dizer "atrair" e "ser invariante" pela ação do semigrupo. Com isso, sabemos o que é um atrator global.

Definição 1.29. Dizemos que $\mathcal{A} \subset X$ é um atrator global para $T(\cdot)$, ou simplesmente um atrator, quando é compacto, invariante e atrai cada um dos conjuntos limitados de $X$ pela ação de $T(\cdot)$.

Não fica claro, a princípio, se um semigrupo $T(\cdot)$ pode ter mais de um atrator global. Isto de fato não ocorre: o atrator é o menor compacto invariante que atrai os limitados de $X$.

Sejam $\mathcal{A}_{1}$ e $\mathcal{A}_{2}$ atratores globais para $T(\cdot)$. Uma vez que $\mathcal{A}_{1}$ é limitado invariante e $\mathcal{A}_{2}$ é atrator, segue que

$$
\operatorname{dist}_{\mathrm{H}}\left(\mathcal{A}_{1}, \mathcal{A}_{2}\right)=\operatorname{dist}_{\mathrm{H}}\left(T(t) \mathcal{A}_{1}, \mathcal{A}_{2}\right) \stackrel{t \rightarrow \infty}{\longrightarrow} 0
$$

de modo que $\mathcal{A}_{1} \subset \overline{\mathcal{A}_{2}}=\mathcal{A}_{2}$; e analogamente $\mathcal{A}_{2} \subset \mathcal{A}_{1}$.

Uma vez verificada a unicidade, a notação $\mathcal{A}$ fica então reservada para o atrator do semigrupo $T(\cdot)$. Denotando por $\mathcal{I B}$ a coleção dos conjuntos limitados invariantes de $X$, ocorre que, se $T(\cdot)$ tem um atrator global $\mathcal{A}$, então

$$
\mathcal{A}=\bigcup_{B \in \mathcal{I} \mathcal{B}} B
$$

De fato, uma das inclusões já é válida, pois $\mathcal{A} \in \mathcal{I} \mathcal{B}$. Agora, dado $B \in \mathcal{I} \mathcal{B}$, então $\operatorname{dist}_{\mathrm{H}}(B, \mathcal{A})=\operatorname{dist}_{\mathrm{H}}(T(t) B, \mathcal{A}) \stackrel{t \rightarrow \infty}{\longrightarrow} 0$, consequentemente $B \subset \mathcal{A}$.

As soluções globais podem então ser usadas para caracterizar um atrator: $\mathcal{A}$ é o conjunto das órbitas globais limitadas de $T(\cdot)$. De fato, sendo $\mathcal{A}$ invariante, sabemos que é uma reunião de todas as órbitas globais contidas em $\mathcal{A}$, consequentemente limitadas, mostrando uma das inclusões. Reciprocamente, $\gamma(\xi)$ é uma órbita limitada, então $\gamma(\xi) \in$ $\mathcal{I B}$ e como sabemos, isto implica que $\gamma(\xi) \in \mathcal{A}$. Ou seja,

$$
\mathcal{A}=\{x \in X \text { : existe uma solução global limitada passando por } x\}
$$

A fim de investigar sob que condições um semigrupo admite um atrator global, vamos precisar de mais algumas definições que envolvem a dinâmica assintótica que conjuntos (primordialmente os limitados de $X$ ) sofrem sob a ação de $T(\cdot)$. 
Dados $x \in X, B \subset X$ e $\tau \geqslant 0$, definimos a semi-órbita positiva de $x$ por

$$
\gamma^{+}(x)=\{T(t) x: t \geqslant 0\}
$$

a semi-órbita positiva de $B$ por

$$
\gamma^{+}(B)=\bigcup_{x \in B} \gamma^{+}(x)=\{T(t) x: t \geqslant 0, x \in B\}
$$

e a semi-órbita positiva de $B$ à direita de $\tau$ por

$$
\gamma_{\tau}^{+}(B)=\gamma^{+}(T(\tau) B)
$$

Estas noções de órbita são os ingredientes necessários para definir o conjunto $\omega$-limite e demonstrar as boas propriedades topológicas que possui. Este é um objeto de grande valor quando se tem a intenção de estudar atratores, uma vez que com ele vamos caracterizar os semigrupos que têm atrator global: os que são assintoticamente compactos e dissipativos (conceitos a serem dados a seu tempo).

Daqui para frente, a notação $t_{n} \nearrow \infty$ indica que $\left(t_{n}\right)_{n}$ é uma sequência de números reais não negativos crescente que diverge para $\infty$. Bem como $t_{n} \searrow-\infty$ indica que $\left(t_{n}\right)_{n}$ é uma sequência de números reais não positivos decrescente que diverge para $-\infty$. Mais geralmente, reservamos a notação $\left(x_{n}\right)_{n \in \mathbb{N}}$, ou simplesmente $\left(x_{n}\right)_{n}$, para nos referirmos a sequências, enquanto que $\left\{x_{n}\right\}_{n \in \mathbb{N}},\left\{A_{\lambda}\right\}_{\lambda \in \Lambda}$ e $\left\{f_{\lambda}\right\}_{\lambda \in \Lambda}$ indicam uma coleção de pontos, conjuntos e funções, respectivamente.

Assim como no estudo qualitativo de equações diferenciais ordinárias, em que o conceito de ciclo limite estável surge, podemos fazer um paralelo e dar alguma intuição ao conceito de $\omega$-limite inspirados nos ciclos limite.

Exemplo 1.30. Considere a equação de van der Pol

$$
u^{\prime \prime}-\lambda\left(1-u^{2}\right) u^{\prime}+u=0, \operatorname{com} \lambda>0,
$$

a qual descreve oscilações autossuficientes em que a energia é alimentada quando há pequenas oscilações e é enfraquecida quando há fortes oscilações. Motivada pelo estudo de circuitos em que os tubos são considerados no vácuo. 
Alternativamente, pondo $\lambda=1 / \varepsilon$,

$$
\left\{\begin{array}{l}
\varepsilon u^{\prime}=v-\frac{u^{3}}{3}+u \\
v^{\prime}=-\varepsilon u
\end{array},\right.
$$

a qual possui um único ciclo limite assintoticamente estável, para cada $\varepsilon>0$.

O seu retrato de fase é dado por

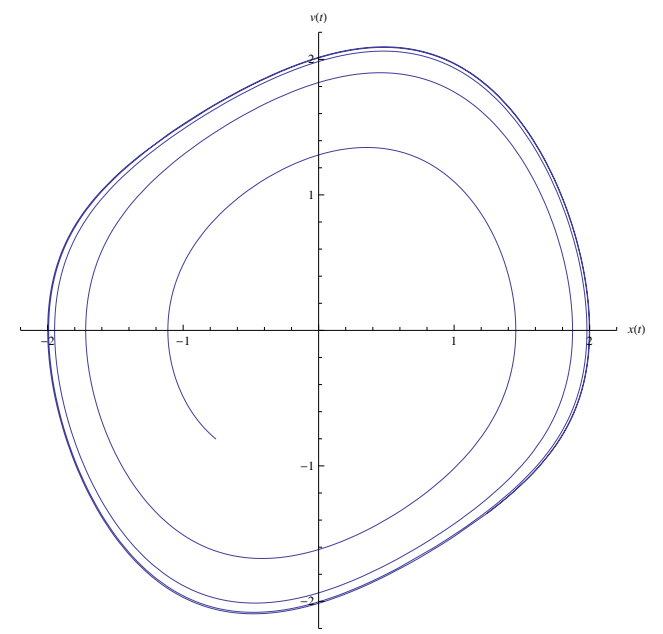

Figura 1.1: Ciclo limite do Oscilador de van der Pol.

O conjunto $\omega$-limite de um dado $B \subset X$, denotado por $\omega(B)$, é definido por

$$
\omega(B):=\bigcap_{t \geqslant 0} \overline{\gamma_{t}^{+}(B)}
$$

ou equivalentemente,

$$
\omega(B)=\left\{x \in X: \text { existem } t_{j} \nearrow \infty \text { e }\left(x_{j}\right)_{j} \subset B \text { tais que } x=\lim _{j \rightarrow \infty} T\left(t_{j}\right) x_{j}\right\} .
$$

De fato, dado $x \in \bigcap_{t \geqslant 0} \overline{\gamma_{t}^{+}(B)}$, temos em particular que $x \in \bigcap_{j \in \mathbb{N}} \overline{\gamma_{j}^{+}(B)}$, daí para cada $j$, existem $z_{j} \in \gamma_{j}^{+}(B)=\bigcup_{t \geqslant j} T(t) B$ e $t_{j} \geqslant j$ tais que $\mathrm{d}\left(x, z_{j}\right)<1 / j$ e $z_{j}=T\left(t_{j}\right) x_{j}$, para $\operatorname{algum} x_{j} \in B$. Comod $\left(x, T\left(t_{j}\right) x_{j}\right)<1 / j$, segue que $x=\lim _{j \rightarrow \infty} T\left(t_{j}\right) x_{j}$. 
Reciprocamente, se para $x \in X$ existem $t_{j} \nearrow \infty \mathrm{e}\left(x_{j}\right)_{j} \subset B$ tais que $x=\lim _{j \rightarrow \infty} T\left(t_{j}\right) x_{j}$, então dado $t \geqslant 0$, existe $j(t)$ natural tal que $t_{j} \geqslant t, \forall j \geqslant j(t)$. Daí, $T\left(t_{j}\right) x_{j}$ está em $\gamma_{t}^{+}(B)$ sempre que $j \geqslant j(t)$. Sendo $x$ o limite destes $T\left(t_{j}\right) x_{j}$, segue que $x \in \overline{\gamma_{t}^{+}(B)}$.

De imediato, vemos que

1) $\omega(B)$ é um conjunto fechado;

2) se $B$ é invariante, então $\omega(B)=\bar{B}$;

3) $\omega(B \cup C) \subset \omega(B) \cup \omega(C)$;

4) $\omega(B) \subset \omega(C)$ sempre que $B \subset C$;

5) $\omega(T(t) B)=\omega(B)$, para todos os instantes $t \in \mathbb{R}$ e subconjuntos $B \subset X$.

Em particular,

$$
\omega(\xi(t))=\omega(\xi(s))
$$

para todo os $t, s \in \mathbb{R}$.

Um semigrupo $T(\cdot)$ é dito assintoticamente compacto quando, dadas $\left(x_{n}\right)_{n} \subset X$ limitada e $t_{n} \nearrow \infty$, tem-se que a sequência $\left(T\left(t_{n}\right) x_{n}\right)_{n} \subset X$ admite uma subsequência convergente.

Lema 1.31. Sejam $B \subset X$ limitado não vazio e $T(\cdot)$ um semigrupo assintoticamente compacto, então

(I) $\omega(B)$ é não vazio, compacto, invariante e atrai B pela ação de $T(\cdot)$;

(II) $\omega(B)$ é o menor fechado de $X$ que atrai $B$.

Além disso, se existe um conexo $C \supset B$ que é atraído por $\omega(B)$, então $\omega(B)$ é conexo. Em particular, se B é conexo, então $\omega(B)$ é conexo.

\section{Demonstração:}

$\omega(B) \neq \varnothing$

Dadas $t_{n} \nearrow \infty$ e $\left(x_{n}\right)_{n} \subset B$, como $T(\cdot)$ é assintoticamente compacto, segue que $\left(T\left(t_{n}\right) x_{n}\right)_{n}$ admite uma subsequência $\left(T\left(t_{n_{j}}\right) x_{n_{j}}\right)_{j}$ convergente, digamos para $x \in X$. Por definição, $x \in \omega(B)$. 
$\omega(B)$ é compacto

Basicamente, o argumento que utilizaremos é o de tomar uma subsequência na diagonal. Dada $\left(z_{n}\right)_{n} \subset \omega(B)$, para cada $n$, existem $t_{j}^{(n)} \nearrow \infty$ e $\left(x_{j}^{(n)}\right)_{j} \subset B$ tais que

$$
z_{n}=\lim _{j \rightarrow \infty} T\left(t_{j}^{(n)}\right) x_{j}^{(n)}
$$

Para cada $n$, tome $j_{n}$ natural tal que $j_{n} \geqslant n$ e $\mathrm{d}\left(z_{n}, T\left(t_{j_{n}}^{(n)}\right) x_{j_{n}}^{(n)}\right)<1 / n$ e como $T(\cdot)$ é assintoticamente compacto, a sequência $\left(T\left(t_{j_{n}}^{(n)}\right) x_{j_{n}}^{(n)}\right)_{n}$ possui uma subsequência convergente, digamos para $z \in X$, a qual indicaremos da mesma maneira:

$$
z=\lim _{n \rightarrow \infty} T\left(t_{j_{n}}^{(n)}\right) x_{j_{n}}^{(n)}
$$

e então

$$
\begin{aligned}
\mathrm{d}\left(z_{n}, z\right) & \leqslant \mathrm{d}\left(z_{n}, T\left(t_{j_{n}}^{(n)}\right) x_{j_{n}}^{(n)}\right)+\mathrm{d}\left(T\left(t_{j_{n}}^{(n)}\right) x_{j_{n}}^{(n)}, z\right) \\
& <1 / n+\mathrm{d}\left(T\left(t_{j_{n}}^{(n)}\right) x_{j_{n}}^{(n)}, z\right) \stackrel{n \rightarrow \infty}{\longrightarrow} 0
\end{aligned}
$$

de modo que $z \in \omega(B)$ é limite de uma subsequência de $\left(z_{n}\right)_{n}, \operatorname{logo} \omega(B)$ é compacto.

$$
\omega(B) \text { é invariante }
$$

Fixados $x \in \omega(B)$ e $t \geqslant 0$, existem $t_{n} \nearrow \infty$ e $\left(x_{n}\right)_{n} \subset B$ tais que

$$
x=\lim _{n \rightarrow \infty} T\left(t_{n}\right) x_{n}
$$

Note que $T(t) x=\lim _{n \rightarrow \infty} T\left(t+t_{n}\right) x_{n}, \log 0 T(t) x \in \omega(B)$.

Para mostrar que $\omega(B) \subset T(t) \omega(B)$, note que podemos escrever $x=\lim T\left(t_{n}\right) x_{n}=$ $\lim T(t)\left(T\left(t_{n}-t\right) x_{n}\right)$ e novamente pela compacidade assintótica, existe $\left(T\left(t_{n_{j}}-t\right) x_{n_{j}}\right)_{j}$ convergente, digamos para $z \in \omega(B)$, daí

$$
x=\lim _{j \rightarrow \infty} T(t)\left(T\left(t_{n_{j}}-t\right) x_{n_{j}}\right)=T(t)\left(\lim _{j \rightarrow \infty} T\left(t_{n_{j}}-t\right) x_{n_{j}}\right)=T(t) z
$$

o que implica que $x \in T(t) \omega(B)$.

$$
\omega(B) \text { atrai } B
$$


Se não fosse assim, existiriam $\varepsilon>0$ e $t_{n} \nearrow \infty$ tais que

$$
\operatorname{dist}_{\mathrm{H}}\left(T\left(t_{n}\right) B, \omega(B)\right) \geqslant \varepsilon, \forall n \in \mathbb{N} \text {. }
$$

Por definição de semidistância de Hausdorff, para cada $n$, existe $x_{n} \in B$ tal que $\mathrm{d}\left(T\left(t_{n}\right) x_{n}, \omega(B)\right) \geqslant \varepsilon$. Novamente passando a uma subsequência de $\left(T\left(t_{n}\right) x_{n}\right)_{n}$ convergente para $x \in \omega(B)$, obtemos $\mathrm{d}(x, \omega(B)) \geqslant \varepsilon$, um absurdo!

$\omega(B)$ é o menor fechado que atrai $B$

Seja $F \subset X$ fechado que atrai $B$ e suponha que $\omega(B) \nsubseteq F$, i.e., existe $x \in \omega(B) \backslash F$, daí $\mathrm{d}(x, F)>\delta>0$.

Como $x=\lim T\left(t_{n}\right) x_{n}, \operatorname{com} t_{n} \nearrow \infty \mathrm{e}\left(x_{n}\right)_{n} \subset B$, existe $t_{0}>0$ tal que $\operatorname{dist}_{\mathrm{H}}(T(t) B, F)<$ $\delta / 2, \forall t \geqslant t_{0}$.

Podemos assumir que $t_{n} \geqslant t_{0}, \forall n$, daí

$$
\delta / 2>\operatorname{dist}_{\mathrm{H}}\left(T\left(t_{n}\right) B, F\right) \geqslant \operatorname{dist}_{\mathrm{H}}\left(T\left(t_{n}\right) x_{n}, F\right)
$$

e passando o limite com $n \rightarrow \infty$, obtemos $\mathrm{d}(x, F) \leqslant \delta / 2$, uma contradição!

Logo, $\omega(B) \subset F$.

Quanto à conexidade, suponha $C \supset B$ conexo que é atraído por $\omega(B)$.

Suponha por absurdo que $\omega(B)$ é desconexo: $\omega(B)=F_{1} \cup F_{2}$, com $F_{1}$ e $F_{2}$ conjuntos fechados disjuntos não vazios.

Uma vez que $F_{1}$ e $F_{2}$ são compactos disjuntos, tem-se $\mathrm{d}\left(F_{1}, F_{2}\right)>\delta>0$. Tome $\tau \geqslant 0$ tal que $T(t) C \subset \mathcal{O}_{\delta / 2}(\omega(B)), \forall t \geqslant \tau$, logo

$$
\gamma_{\tau}^{+}(C) \subset \mathcal{O}_{\delta / 2}(\omega(B))=\mathcal{O}_{\delta / 2}\left(F_{1}\right) \cup \mathcal{O}_{\delta / 2}\left(F_{2}\right),
$$

uma reunião disjunta.

Note que $\gamma_{\tau}^{+}(C)$ é a imagem de $[\tau, \infty) \times C$ pela aplicação contínua $(t, x) \longmapsto T(t) x, \operatorname{logo}$ $\gamma_{\tau}^{+}(C)$ é conexo e então está contido em uma das vizinhanças acima, digamos $\gamma_{\tau}^{+}(C) \subset$ $\mathcal{O}_{\delta / 2}\left(F_{1}\right)$, mas

$$
F_{2} \subset \omega(B) \subset \overline{\gamma_{\tau}^{+}(B)} \subset \overline{\gamma_{\tau}^{+}(C)} \subset \overline{\mathcal{O}_{\delta / 2}\left(F_{1}\right)},
$$

uma contradição, pois $\mathrm{d}\left(F_{1}, F_{2}\right)>\delta$. 
Com isso, se $T(\cdot)$ é assintoticamente compacto e $B \subset X$ é limitado invariante, então $\bar{B}=\omega(B)$ também é.

Neste contexto faz sentido definir os conjuntos $\alpha$-limite e $\omega$-limite da solução $\xi$. Dado $t \in \mathbb{R}$, definimos o $\omega$-limite da solução $\xi: \mathbb{R} \rightarrow X$ por $\omega(\xi(t))$ e denotamos por $\omega(\xi)$. E o $\alpha$-limite da solução $\xi$ é definido por

$$
\alpha(\xi)=\left\{x \in X: \text { existe } t_{j} \searrow-\infty \operatorname{com} x=\lim _{j \rightarrow \infty} \xi\left(t_{j}\right)\right\} .
$$

O lema anterior tem sua formulação análoga para o conjunto $\alpha$-limite. Note que esta noção é mais adequada para grupos $\{T(t): t \in \mathbb{R}\}$, pois faz sentido considerar instantes $t$ arbitrariamente negativos. Além disso, se por um lado há unicidade de solução para $t$ no futuro, não há para $t$ no passado, i.e., $T(t)^{-1} x$ pode não ser unitário de modo que podem existir diferentes soluções $\xi_{j}$ que levam pontos $x_{j}$ a $x$ em tempo $t$.

Para caracterizar semigrupos que possuem atrator, são necessários os conceitos de absorção e dissipatividade.

Definição 1.32. Dados $B, D \subset X$, dizemos que $D$ absorve $B$ pela ação de $T(\cdot)$, quando existe $\tau=\tau(B, D) \geqslant 0$ tal que $T(t) B \subset D$, para todo $t \geqslant \tau$.

Vale observar que, se $D$ absorve $B$, então

a) $\bar{D}$ também absorve $B$, de modo que sempre podemos assumir que $D$ é fechado;

b) $D$ atrai $B$, e consequentemente, $\mathcal{O}_{\delta}(D)$ absorve $B$, qualquer que seja $\delta>0$.

E é da noção de absorção que vem a noção de dissipatividade.

Definição 1.33. Um semigrupo $T(\cdot)$ é dito limitado dissipativo, ou simplesmente um semigrupo dissipativo, quando existe um limitado (fechado) $D \subset X$ que absorve todos os subconjuntos limitados de $X$

Teorema 1.34. Um semigrupo $T(\cdot)$ possui atrator global $\mathcal{A}$ se, e somente se, $T(\cdot)$ é assintoticamente compacto e dissipativo.

Em caso afirmativo, tem-se

$$
\mathcal{A}=\bigcup_{B \in \mathcal{B}} \omega(B)
$$

em que $\mathcal{B}$ denota a coleção dos limitados de $X$. 


\section{Demonstração:}

Seja $\mathcal{A}$ o atrator de $T(\cdot)$. Sabidamente, temos que $T(\cdot)$ é um semigrupo dissipativo. Por outro lado, sejam $t_{n} \nearrow \infty$ e $\left(x_{n}\right)_{n} \subset X$ limitada, e considere $B=\left\{x_{n}: n \in \mathbb{N}\right\}$, daí

$$
\lim _{t \rightarrow \infty} \operatorname{dist}_{\mathrm{H}}(T(t) B, \mathcal{A})=0
$$

e em particular $\lim _{n \rightarrow \infty} \operatorname{dist}_{\mathrm{H}}\left(T\left(t_{n}\right) B, \mathcal{A}\right)=0$.

Assim, para cada $j \in \mathbb{N}$, existe $n_{j} \geqslant j$ tal que $\operatorname{dist}_{\mathrm{H}}\left(T\left(t_{n_{j}}\right) B, \mathcal{A}\right)<1 / j$, de modo que $\mathrm{d}\left(T\left(t_{n_{j}}\right) x_{n_{j}}, \mathcal{A}\right)<1 / j$ e, por definição de ínfimo, temos $\mathrm{d}\left(T\left(t_{n_{j}}\right) x_{n_{j}}, z_{j}\right)<1 / j$, para $\operatorname{algum} z_{j} \in \mathcal{A}$.

Da compacidade de $\mathcal{A}$, podemos assumir que $\left(z_{j}\right)_{j}$ converge para $z \in \mathcal{A}$, logo

$$
\mathrm{d}\left(T\left(t_{n_{j}}\right) x_{n_{j}}, z\right) \leqslant \mathrm{d}\left(T\left(t_{n_{j}}\right) x_{n_{j}}, z_{j}\right)+\mathrm{d}\left(z_{j}, z\right)<1 / j+\mathrm{d}\left(z_{j}, z\right) \stackrel{j \rightarrow \infty}{\longrightarrow} 0 .
$$

Reciprocamente, supondo $T(\cdot)$ um semigrupo assintoticamente compacto e dissipativo, seja $D \subset X$ um fechado limitado que absorve cada um dos subconjuntos limitados de $X$ e defina

$$
\mathcal{A}=\bigcup_{B \in \mathcal{B}} \omega(B)
$$

o qual é invariante por ser uma reunião de invariantes.

Note ainda que $\mathcal{A}$ atrai cada um dos limitados de $X$ : se $B \in \mathcal{B}$, então

$$
\operatorname{dist}_{\mathrm{H}}(T(t) B, \mathcal{A}) \leqslant \operatorname{dist}_{\mathrm{H}}(T(t) B, \omega(B)) \stackrel{t \rightarrow \infty}{\longrightarrow} 0 .
$$

Dado $B \in \mathcal{B}$, como $D$ atrai $B$ e $\omega(B)$ é o menor fechado que atrai $B$, segue que

$$
D \supset \bigcup_{B \in \mathcal{B}} \omega(B)=\mathcal{A} .
$$

Uma vez que $D$ é fechado limitado, temos

$$
\mathcal{A} \subset \overline{\mathcal{A}}=\omega(\overline{\mathcal{A}}) \subset \omega(D) \subset \mathcal{A},
$$

de modo que $\mathcal{A}$ coincide com o compacto $\omega(D)$.

Portanto, $\mathcal{A}$ é um atrator global. 
Note que pela demonstração do Teorema acima, a fim de mostrar que um semigrupo $T(\cdot)$ tem atrator global, basta encontrar um compacto que atrai os limitados de $X$.

Veremos que todo semigrupo eventualmente compacto, eventualmente limitado e ponto dissipativo possui atrator global; e esta é uma condição mais fácil de se verificar na prática.

Definição 1.35. Um semigrupo $T(\cdot)$ é dito

a) limitado quando, para todo conjunto limitado $B$, tem-se que $\gamma^{+}(B) \subset X$ é limitado;

b) eventualmente limitado quando, para todo conjunto limitado $B$, existe $\tau=\tau(B)$ tal que $\gamma_{\tau}^{+}(B) \subset X$ é limitada;

c) ponto dissipativo quando existe um limitado $D \subset X$ que absorve todos os pontos de $X$;

d) eventualmente compacto quando existe $t_{0} \geqslant 0$ tal que $T\left(t_{0}\right): X \longrightarrow X$ é uma aplicação compacta, i.e., leva limitados de $X$ em pré-compactos de $X$.

Nem todo semigrupo ponto dissipativo é dissipativo, simplesmente porque, dado um limitado $B \supset\left\{x_{1}, x_{2}, \ldots\right\}$, pode ser que cada um dos pontos $x_{n}$ "demore" cada vez mais a ser absorvido, de modo que $D$ absorve cada um dos pontos de $x_{n}$ em tempo finito, mas não $\left\{x_{1}, x_{2}, \ldots\right\}$ como um todo.

Exemplo 1.36. Seja $X=l^{2}:=\left\{\left(x_{n}\right)_{n}: x_{n}\right.$ real e $\left.\sum_{n=1}^{\infty} x_{n}^{2}<\infty\right\}$ e considere o semigrupo discreto em $X$ obtido da aplicação $T: l^{2} \rightarrow l^{2}$, dada por

$$
T\left(\left(x_{n}\right)_{n}\right)=\left(x_{2}, x_{3}, x_{4}, \ldots\right) .
$$

Não é difícil ver que, dado $\left(x_{n}\right)_{n} \in l^{2}$, as iteradas de $T$ convergem para a sequência nula, a qual é o atrator deste semigrupo, o que mostra a dissipatividade pontual.

Por outro lado, a coleção das sequências $\left\{e_{j}: j \in \mathbb{N}\right\}$, em que $e_{1}=(1,0,0, \ldots), e_{2}=$ $(0,1,0,0, \ldots), e_{3}=(0,0,1,0,0, \ldots), \ldots$ em $S_{X}[0,1]=\left\{\left(x_{n}\right)_{n} \in l^{2}: \sum_{n=1}^{\infty} x_{n}^{2}=1\right\}$, é invariante por $T$ e não converge para o atrator. Logo, $T(\cdot)$ não é limitado dissipativo.

Observação 1.37. Vale constatar que

a) todo semigrupo $T(\cdot)$ com atrator $\mathcal{A}$ é eventualmente limitado e dissipativo, basta considerar $D=\mathcal{O}_{1}(\mathcal{A})$; 
b) se $T(\cdot)$ é um semigrupo eventualmente compacto, então $T(t): X \longrightarrow X$ é uma aplicação compacta, para todo $t \geqslant t_{0}$, por ser composição de aplicação compacta com aplicação contínua;

c) se $T(\cdot)$ é eventualmente compacto e eventualmente limitado, então é assintoticamente compacto.

De fato, dadas $t_{n} \nearrow \infty e\left(x_{n}\right)_{n} \subset X$ limitada, tem-se que $B_{0}=\left\{x_{n}: n \in \mathbb{N}\right\}$ é limitado em $X$, dai existe $\tau>0$ tal que $\gamma_{\tau}^{+}\left(B_{0}\right)$ é limitada. Sejam $t_{0}>0$ e $n_{0} \in \mathbb{N}$ tais que $T\left(t_{0}\right): X \rightarrow X$ é compacta e $t_{n} \geqslant t_{0}+\tau$, para todo $n \geqslant n_{0}$.

Com isso, $B=\left\{T\left(t_{n}-t_{0}\right) x_{n}: n \geqslant n_{0}\right\}$ é limitado, pois $B \subset \gamma_{\tau}^{+}\left(B_{0}\right)$. Como $T\left(t_{0}\right) B$ é pré-compacto, segue que $\left(T\left(t_{n}\right) x_{n}\right)_{n}$ admite uma subsequência convergente.

Corolário 1.38. Se T(.) é eventualmente compacto, eventualmente limitado e ponto dissipativo, então possui um atrator global.

\section{Demonstração:}

Sabemos que $T(\cdot)$ é um semigrupo assintoticamente compacto. Pelo Teorema 1.34, basta mostrar a dissipatividade do semigrupo.

Seja $D_{0} \subset X$ um limitado que absorve cada ponto de $X$ e considere o limitado aberto $D_{1}=\mathcal{O}_{1}\left(D_{0}\right)$, para o qual existe um instante $\tau>0$ de modo que $D=\gamma_{\tau}^{+}\left(D_{1}\right)$ é limitado, graças à limitação eventual do semigrupo.

Fixado $K \subset X$ compacto, para cada ponto $x \in K$, existe $\tau_{x}>0$ de maneira que $T(t) x \in D_{1}, \forall r \geqslant \tau_{x}$.

Uma vez que $T\left(\tau_{x}\right): X \rightarrow X$ é contínua, existe $\delta_{x}>0$ tal que $T\left(\tau_{x}\right) \mathcal{O}_{\delta_{x}}(x) \subset D_{1}$

$$
\begin{aligned}
& \Rightarrow T(t)\left(T\left(\tau_{x}\right) \mathcal{O}_{\delta_{x}}(x)\right) \subset T(t) D_{1}, \forall t \geqslant 0 \\
& \Rightarrow T(t)\left(T\left(\tau_{x}\right) \mathcal{O}_{\delta_{x}}(x)\right) \subset D, \forall t \geqslant \tau \\
& \Rightarrow T(t) \mathcal{O}_{\delta_{x}}(x) \subset D, \forall t \geqslant \tau+\tau_{x} .
\end{aligned}
$$

Da compacidade de $K$, tome pontos $x_{1}, \ldots, x_{n} \in K$ e respectivos $\delta_{1}, \ldots, \delta_{n}$ tais que $K \subset \bigcup_{j=1}^{n} \mathcal{O}_{\delta_{j}}\left(x_{j}\right)$, e pondo $\tau_{K}=\max _{j=1, \ldots, n} \tau_{x_{j}}$, segue que

$$
T(t) K \subset \bigcup_{j=1}^{n} T(t) \mathcal{O}_{\delta_{j}}\left(x_{j}\right) \subset D
$$


para todo $t \geqslant t+\tau_{K}$.

Por fim, dados $B \subset X$ limitado e $t_{0}$ tal que $T\left(t_{0}\right): X \rightarrow X$ é uma aplicação compacta, temos que $K:=\overline{T\left(t_{0}\right) B}$ é compacto. E agora, como $T(t)\left(T\left(t_{0}\right) B\right) \subset T(t) K \subset D$ para todo $t \geqslant \tau+\tau_{K}$, concluimos que

$$
T(t) B \subset D,
$$

para todo $t \geqslant t_{0}+\tau+\tau_{K}$, o que garante a dissipatividade do semigrupo $T(\cdot)$.

Por fim, apresentamos um resultado de existência de atrator sob as mais fracas condições possível, o que o torna uma ferramenta mais fácil de ser usada na prática.

Teorema 1.39. (Carvalho [8], Teorema 1.1.2) Se $X$ é um espaço de Banach e T(·) é um semigrupo assintoticamente compacto, eventualmente limitado e ponto dissipativo, então $T(\cdot)$ possui um atrator global $\mathcal{A}$.

\subsubsection{Semigrupos Gradientes}

Vamos introduzir uma noção de semigrupos que tem apelo prático para vários problemas físicos e cuja dinâmica é bem conhecida e explorada, os semigrupos gradientes.

Basicamente, um semigrupo gradiente é aquele que admite uma função de Lyapunov $V: X \rightarrow \mathbb{R}$ de tal forma que suas soluções não formem estruturas "recorrentes".

Definição 1.40. Dizemos que um invariante $E \subset X$ é isolado quando é limitado e existe $\delta>0$ tal que E é o invariante maximal de sua $\delta$-vizinhança, i.e., se $F \subset \mathcal{O}_{\delta}(E)$ é um outro invariante, então $F \subset E$.

Importante destacar que um invariante isolado $E$ é, antes de qualquer outra coisa, um invariante limitado, de modo que, se o semigrupo admite um atrator $\mathcal{A}$, então $E \subset \mathcal{A}$. Em particular, $E$ é compacto.

De fato, $E$ maximal em $\mathcal{O}_{\delta}(E)$ implica que $\bar{E} \subset \overline{\mathcal{O}_{\delta / 2}(E)} \subset \mathcal{O}_{\delta}(E)$; uma vez que $\bar{E}$ é invariante, temos $\bar{E} \subset E$, $\log o E \subset \mathcal{A}$ é fechado.

Dados $E_{1}, \ldots, E_{m}$ invariantes isolados disjuntos, dizemos que $\Xi=\left\{E_{1}, \ldots, E_{m}\right\}$ é uma família finita disjunta de invariantes isolados, ou abreviadamente uma F.F.D.I.I., se existe $\delta>0$ tal que $\mathcal{O}_{\delta}\left(E_{i}\right) \cap \mathcal{O}_{\delta}\left(E_{j}\right)=\varnothing$ sempre que $i \neq j$. 
Definição 1.41. Um semigrupo T(.) que possui uma F.F.D.I.I. $\Xi=\left\{E_{1}, \ldots, E_{m}\right\}$ é dito um semigrupo gradiente com respeito $\boldsymbol{a} \Xi$ quando existe uma função contínua $V: X \rightarrow \mathbb{R}$ tal que

i) a aplicação $[0, \infty) \ni t \longmapsto V(T(t) x) \in X$ é não crescente, para todo $x \in X$;

ii) se existe $x \in X$ tal que $V(T(t) x)=V(x)$, para todo $t \geqslant 0$, então $x \in E_{j}$, para algum $j \in\{1, \ldots, m\}$;

iii) $V$ é constante sobre cada $E_{j} \in \Xi$.

Nestas condições, a função $V$ é chamada uma função de Lyapunov para $T(\cdot)$ com respeito a $\Xi$ e usaremos a notação $(T(\cdot), \Xi, V)$ para falar do semigrupo gradiente $T(\cdot)$ com respeito a $\Xi$ com a função de Lyapunov $V$ correspondente.

A propriedade do item $i$ ) nos faz dizer que $V$ é não crescente ao longo de soluções.

Estruturas homoclínicas podem ser entendidas como uma generalização órbitas homoclínicas e acontece que semigrupos gradientes não admitem tais estruturas.

Seja $T(\cdot)$ um semigrupo possuindo uma F.F.D.I.I. $\Xi=\left\{E_{1}, \ldots, E_{m}\right\}$. Uma estrutura homoclínica associada a $\Xi$ consiste de um subconjunto $\Xi^{\prime}=\left\{E_{l_{1}}, \ldots, E_{l_{k}}\right\} \subset \Xi$ e de soluções globais $\xi_{1}, \ldots, \xi_{k}$ de modo que, para todo $j=1, \ldots, k$, valem

i) existe $t_{j} \in \mathbb{R}$ tal que $\xi_{j}\left(t_{j}\right) \notin E_{l_{j}} \cup E_{l_{j+1}}$; (pondo $E_{l_{k+1}}=E_{l_{1}}$ )

ii) $\lim _{t \rightarrow-\infty} \mathrm{d}\left(\xi_{j}(t), E_{l_{j}}\right)=0$ e $\lim _{t \rightarrow \infty} \mathrm{d}\left(\xi_{j}(t), E_{l_{j+1}}\right)=0$.

Note que se $k>1$, então a condição $i i$ ) implica a $i$ ). Porém, para contemplar o caso $k=1$, o item $i$ ) é de fato relevante. O item $i i$ ) diz que a órbita de $\xi_{j}$, a partir de um certo instante, não sai da $\delta$-vizinhança de $E_{l_{j+1}}$; em particular, fica "distante" de outros invariantes isolados, o que de certa forma justifica a denominação.

Costumeiramente, escrevemos $\xi_{j}(t) \stackrel{t \rightarrow-\infty}{\longrightarrow} E_{l_{j}}$ e $\xi_{j}(t) \stackrel{t \rightarrow \infty}{\longrightarrow} E_{l_{j+1}}$ para descrever o item ii); ou ainda mais simplificadamente,

$$
E_{l_{j}} \stackrel{t \rightarrow-\infty}{\longleftarrow} \xi_{j}(t) \stackrel{t \rightarrow \infty}{\longrightarrow} E_{l_{j+1}} .
$$

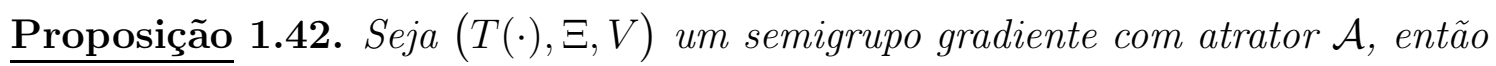


G1) se $\xi: \mathbb{R} \rightarrow X$ é uma solução limitada, então existem $i$ e $j$ em $\{1, \ldots, m\}$ tais que

$$
E_{i} \stackrel{t \rightarrow-\infty}{\longleftarrow} \xi(t) \stackrel{t \rightarrow \infty}{\longrightarrow} E_{j}
$$

G2) não existem estruturas homoclinicas associadas a $\Xi$.

\section{Demonstração:}

Seja $\xi$ uma solução limitada de $T(\cdot)$. Como $\gamma(\xi) \subset \mathcal{A}$ e $\mathbb{R} \ni t \longmapsto V(\xi(t)) \in \mathbb{R}$ é contínua, não crescente e limitada, existem os limites

$$
\lim _{t \rightarrow \infty} V(\xi(t))=l \leqslant L=\lim _{t \rightarrow-\infty} V(\xi(t)) .
$$

Em particular, $\left.V\right|_{\alpha(\xi)} \equiv L$ e $\left.V\right|_{\omega(\xi)} \equiv l$.

Mostremos que $\omega(\xi) \subset E_{j}$, para algum $j \in\{1, \ldots, m\}$. Dado $x \in \omega(\xi)$, uma vez que $T(t) x \in \omega(\xi)$, para todo $t \geqslant 0$, temos

$$
l=V(x)=V(T(t) x), \forall t \geqslant 0,
$$

e então, da definição de semigrupo gradiente, $x \in E_{j}$, para algum $j=j(x)$. Mas como $\omega(\xi)$ é conexo, segue que $\omega(\xi) \subset E_{j}$, o que implica que

$$
\lim _{t \rightarrow \infty} \mathrm{d}\left(\xi(t), E_{j}\right)=0
$$

De maneira análoga, prova-se que $\alpha(\xi) \subset E_{i}$, para algum $i$.

Quanto ao item $i i)$, vamos proceder por absurdo: sejam $\Xi^{\prime}=\left\{E_{l_{1}}, \ldots, E_{l_{k}}\right\}$ e $\xi_{1}, \ldots, \xi_{k}$ uma estrutura homoclínica associada a $\Xi$; e tome constantes $L_{1}, \ldots, L_{k}, L_{k+1}=L_{1}$ tais que $\left.V\right|_{E_{l_{j}}} \equiv L_{j}$, para cada $j \in 1, \ldots, k+1$ (da definição de semigrupo gradiente).

Assim, fixados $j \in\{1, \ldots, k+1\}, s \in \mathbb{R}$ e $t \geqslant 0$, como $L_{k+1}=L_{1}$ e

$$
L_{j} \geqslant V\left(\xi_{j}(s)\right) \geqslant V\left(T(t) \xi_{j}(s)\right) \geqslant L_{j+1}
$$

obtemos $L_{j}=: L$, para todo $j$, e ainda $V\left(\xi_{j}(s)\right)=V\left(T(t) \xi_{j}(s)\right)$. Pela definição de semigrupo gradiente, pela invariância $\operatorname{dos} E_{j}$ e dada a arbitrariedade de $s$, segue que $\gamma\left(\xi_{j}\right) \subset E_{p}$, para algum $p \in\{1, \ldots, k\}$. 
Mas sabendo que $\Xi$ é uma F.F.D.I.I. e

$$
E_{l_{j}} \stackrel{t \rightarrow-\infty}{\longleftarrow} \xi_{j}(t) \stackrel{t \rightarrow \infty}{\longrightarrow} E_{l_{j+1}}
$$

concluímos que $E_{p}=E_{l_{j}}=E_{l_{j+1}}$. Consequentemente, todos os invariantes de $\Xi$ são iguais a $E_{p}$, e todas as órbitas $\gamma\left(\xi_{j}\right)$ estão contidas em $E_{p}$. Porém, isto viola o item $i$ ) da definição de estrutura homoclínica.

Portanto, $(T(\cdot), \Xi, V)$ não admite estruturas homoclínicas.

As propriedades $G 1$ ) e $G 2$ ) detêm a essência de um semigrupo gradiente, independente da função de Lyapunov. De fato, um semigrupo $T(\cdot)$ com atrator $\mathcal{A}$ e uma F.F.D.I.I. $\Xi=\left\{E_{1}, \ldots, E_{m}\right\}$ é um semigrupo gradiente se, e somente se, satisfaz as propriedades G1) e G2). Em outras palavras, as noções de semigrupo gradiente e semigrupo do tipo gradiente são equivalentes. Consulte o artigo [1] para mais detalhes.

Note que se $\Xi$ é formado por pontos de equilíbrio $z_{1}^{*}, \ldots, z_{m}^{*}$, então $\omega(x)$ é um conjunto unitário $\left\{z_{j}^{*}\right\}$, para cada $x \in X$.

Da Proposição 1.42, vemos que a dinâmica de um semigrupo gradiente $(T(\cdot), \Xi, V)$ com invariantes isolados fica inteiramente determinada pelos $E_{1}, \ldots, E_{m}$. Como é esperado, isto se reflete no atrator global. Para entender de que forma isto ocorre, definimos o conjunto instável de um invariante $E \subset X$ por

$$
W^{u}(E)=\left\{x \in X \text { : existe uma solução } \xi \text { tal que } \xi(0)=x \text { e } \lim _{t \rightarrow-\infty} \mathrm{d}(\xi(t), E)=0\right\}
$$

e descrevemos o atrator $\mathcal{A}$ em termos dos conjuntos $W^{u}\left(E_{1}\right), \ldots, W^{u}\left(E_{m}\right)$.

Teorema 1.43. Se $(T(\cdot), \Xi, V)$ é um semigrupo gradiente com atrator $\mathcal{A}$, então

$$
\mathcal{A}=\bigcup_{j=1}^{m} W^{u}\left(E_{j}\right)
$$

\section{Demonstração:}

Se $x \in \mathcal{A}$, então existe uma solução limitada $\xi$ tal que $\xi(0)=x$; da Proposição 1.42 , existe $E_{j} \in \Xi \mathrm{com} \lim _{t \rightarrow-\infty} \mathrm{d}\left(\xi(t), E_{j}\right)=0, \operatorname{logo} x \in W^{u}\left(E_{j}\right)$.

Por outro lado, se $x \in W^{u}\left(E_{j}\right)$, para algum $j$, então existe uma solução $\xi$ tal que 
$\xi(0)=x$ e $\lim _{t \rightarrow-\infty} \mathrm{d}\left(\xi(t), E_{j}\right)=0$, daí

$$
\xi(t) \in \mathcal{O}_{1}\left(E_{j}\right) \text { sempre que } t \leqslant-\tau
$$

e, por $G 1)$

$$
\xi(t) \in \mathcal{O}_{1}\left(E_{i}\right) \text { sempre que } t \geqslant \tau,
$$

para algum $\tau>0$ e algum $i \in\{1, \ldots, m\}$.

Como $E_{j}, E_{i} \subset \mathcal{A}$ e $\xi([-\tau, \tau])$ é compacto, temos que

$$
\gamma(\xi)=\xi((-\infty,-\tau]) \cup \xi([\tau, \infty)) \cup \xi([-\tau, \tau]) \subset \mathcal{O}_{1}\left(E_{j}\right) \cup \mathcal{O}_{1}\left(E_{i}\right) \cup \xi([-\tau, \tau])
$$

de onde segue que $\xi$ é uma solução limitada, ou seja, $\gamma(\xi) \subset \mathcal{A}$; em particular, $x \in \mathcal{A}$, como queríamos.

Outra propriedade de um semigrupo gradiente é que, a fim de garantir a existência de um atrator global, basta pedir compacidade e limitação eventuais.

Teorema 1.44. Se $(T(\cdot), \Xi, V)$ é um semigrupo gradiente eventualmente compacto $e$ eventualmente limitado, então possui um atrator global $\mathcal{A}$.

\section{Demonstração:}

Do Corolário 1.38, basta mostrar que $T(\cdot)$ é ponto dissipativo.

Para tanto, verifiquemos que

$$
D_{0}:=\bigcup_{j=1}^{m} \mathcal{O}_{1}\left(E_{j}\right)
$$

é um conjunto limitado que absorve pontos de $X$ pela ação de $T(\cdot)$.

Seja $x \in X$. Como $T(\cdot)$ eventualmente compacto, a órbita de $x$ à direita de $t_{0}>0$ é pré-compacta, para algum $t_{0}$; sendo $\gamma_{\left[0, t_{0}\right]}^{+}(x)$ compacto, segue que $\overline{\gamma^{+}(x)}$ é compacto, $\operatorname{logo}$ a aplicação $\mathbb{R} \ni t \longmapsto V(\xi(t)) \in \mathbb{R}$ é contínua, não crescente e limitada, de modo que $\left.V\right|_{\omega(x)} \equiv l$ e $\omega(x) \subset E_{j} \subset D_{0}$, para algum $j \in\{1, \ldots, m\}$, basta argumentar como na demonstração da Proposição 1.42 .

Como $\omega(x) \subset E_{j}$ atrai $x$ pela ação de $T(\cdot)$, obtemos que $\gamma_{t}^{+}(x) \subset \mathcal{O}_{1}\left(E_{j}\right)$, para $t$ suficientemente grande. 
Do Teorema 1.39, podemos enfraquecer as hipóteses do teorema acima e pedir compacidade assintótica no lugar de compacidade eventual.

O estudo de semigrupos gradientes vai muito além (consulte [1] e [6]), por exemplo:

- a equivalência com a noção de semigrupos do tipo gradiente (por meio da Decomposição de Morse);

- a sua estabilidade por perturbações, um resultado muito importante quando se tem a intenção de modelar um fenômeno, pois toda modelagem tem suas imprecisões e ter ciência de que elas não desqualificam os resultados obtidos nos diz que a modelagem matemática é possível.

Por esta razão, podemos omitir a função de Lyapunov e nos referir a um semigrupo gradiente usando a notação $(T(\cdot), \Xi)$.

A citada Decomposição de Morse envolve o conceito de par atrator-repulsor, o qual também será utilizado nas aplicações que apresentaremos no Capítulo 4, mais precisamente quando formos estudar condições para que a dimensão fractal do atrator global de semigrupos gradientes seja finita.

Definição 1.45. Um conjunto $A \subset \mathcal{A}$ é dito um atrator local para $T(\cdot)$ quando existe $\delta>0$ tal que

$$
A=\omega\left(\mathcal{O}_{\delta}(A)\right)
$$

Neste caso, o seu repulsor (complementar), denotado por $A^{*}$, é definido por

$$
A^{*}=\{x \in \mathcal{A}: \omega(x) \cap A=\varnothing\} \subset \mathcal{A} \text {. }
$$

O par $\left(A, A^{*}\right)$ é dito um par atrator-repulsor para o semigrupo $(T(\cdot), \Xi)$. E mais geralmente, a definição se aplica a qualquer semigrupo $T(\cdot)$ com atrator global $\mathcal{A}$.

Lema 1.46. Neste contexto, valem

i) um subconjunto $A \subset \mathcal{A}$ é um atrator local se, e somente se, $A$ é compacto, invariante e atrai $\mathcal{O}_{\delta}(A)$, para algum $\delta>0$;

ii) $A^{*}$ é compacto, invariante e disjunto de $A=\omega\left(\mathcal{O}_{\delta}(A)\right)$; 
iii) se $A=\omega\left(\mathcal{O}_{\delta}(A)\right)$ e $x \in \mathcal{A}$ é tal que $\omega(x) \cap A \neq \varnothing$, então $\omega(x) \subset A$.

\section{Demonstração:}

Item $i)$

Uma vez que $A=\omega\left(\mathcal{O}_{\delta}(A)\right)$, para algum $\delta>0$, e $\mathcal{O}_{\delta}(A)$ é limitado, segue do Lema 1.31 que $\omega\left(\mathcal{O}_{\delta}(A)\right)$ é compacto, invariante e atrai $\mathcal{O}_{\delta}(A)$.

Suponha $A \subset \mathcal{A}$ compacto, invariante que atrai $\mathcal{O}_{\delta}(A)$, para algum $\delta>0$.

Como $\mathcal{O}_{\delta}(A)$ é limitado, sabemos que $\omega\left(\mathcal{O}_{\delta}(A)\right)$ é o menor fechado que atrai $\mathcal{O}_{\delta}$, daí

$$
\omega\left(\mathcal{O}_{\delta}(A)\right) \subset A
$$

Por outro lado, como $A \subset \mathcal{O}_{\delta}(A)$ é invariante e fechado, temos

$$
A=\bar{A}=\omega(A) \subset \omega\left(\mathcal{O}_{\delta}(A)\right)
$$

de onde segue a igualdade: $A=\omega\left(\mathcal{O}_{\delta}(A)\right)$.

Item $i i)$

Mostremos que $A^{*} \subset \mathcal{A}$ é fechado, daí teremos que $A^{*}$ é compacto.

Seja $\left(a_{n}\right)_{n} \subset A^{*}$ tal que $a_{n} \stackrel{n \rightarrow \infty}{\longrightarrow} a \in \mathcal{A}$ e suponha que $a \notin A^{*}$, i.e., existe $y \in \omega(\{a\}) \cap A$, de modo que

$$
y=\lim _{n \rightarrow \infty} T\left(t_{n}\right) a \in A,
$$

para alguma sequência $t_{n} \nearrow \infty$.

Por definição, podemos escrever $A=\omega\left(\mathcal{O}_{\delta}(A)\right)$, para algum $\delta>0$.

Tome $k_{0}$ natural tal que $T\left(t_{k_{0}}\right) a \in \mathcal{O}_{\delta}(A)$. Sabendo que $a_{n} \rightarrow a, \mathcal{O}_{\delta}(A)$ é aberto e $T\left(t_{k_{0}}\right): X \rightarrow X$ é contínua, existe $n_{0}$ natural tal que $T\left(t_{k_{0}}\right) a_{n_{0}} \in \mathcal{O}_{\delta}(A)$, daí

$$
\omega\left(a_{n_{0}}\right)=\omega\left(T\left(t_{k_{0}}\right) a_{n_{0}}\right) \subset \omega\left(\mathcal{O}_{\delta}(A)\right)=A
$$

$\operatorname{mas} a_{n_{0}} \in A^{*}=\{y \in \mathcal{A}: \omega(\{y\}) \cap A=\varnothing\}$, uma contradição!

Portanto, $A^{*}$ é compacto.

Como $\omega(x)=\omega(T(t) x)$, para todo $t \geqslant 0$, segue que

$$
\omega(x) \cap A=\varnothing \Leftrightarrow \omega(T(t) x) \cap A=\varnothing,
$$


de modo que $A^{*}$ é invariante.

Que $A$ e $A^{*}$ são disjuntos, é simples: dado $x \in A, T(t) x \in A$, para todo $t \geqslant 0$, logo $\omega(x) \subset \bar{A}=A$ e $x \notin A^{*}$.

\section{Item $i i i)$}

Seja $y=\lim _{n \rightarrow \infty} T\left(t_{n}\right) x \in \omega(\{x\}) \cap A$ e tome $n_{0}$ natural tal que $T\left(t_{n}\right) x \in \mathcal{O}_{\delta}(A)$ para todo $n \geqslant n_{0}$, daí

$$
\omega\left(T\left(t_{n_{0}}\right) x\right) \subset \omega\left(\mathcal{O}_{\delta}(A)\right)=A
$$

e usando que $\omega(\{x\})=\omega\left(T\left(t_{n_{0}}\right) x\right)$, obtemos que $\omega(\{x\}) \subset A$.

Definição 1.47. Dada uma $\delta$-vizinhança de um invariante isolado $E_{j} \in \Xi$, definimos

a) o conjunto instável local de $E_{j}$, denotado por $W_{\mathrm{loc}}^{u}\left(E_{j}\right)$ como sendo o conjunto dos pontos $x \in \mathcal{O}_{\delta}\left(E_{j}\right)$ para os quais existe uma solução $\xi: \mathbb{R} \rightarrow X$ tal que $\xi(0)=x$, $\xi(t) \in \mathcal{O}_{\delta}\left(E_{j}\right)$, para todo $t \leqslant 0, e$

$$
\xi(t) \stackrel{t \rightarrow-\infty}{\longrightarrow} E_{j}
$$

b) o conjunto estável local de $E_{j}$, denotado por $W_{\mathrm{loc}}^{s}\left(E_{j}\right)$ como sendo o conjunto dos pontos $x \in \mathcal{O}_{\delta}\left(E_{j}\right)$ para os quais existe uma solução $\xi: \mathbb{R} \rightarrow X$ tal que $\xi(0)=x$, $\xi(t) \in \mathcal{O}_{\delta}\left(E_{j}\right)$, para todo $t \geqslant 0, e$

$$
\xi(t) \stackrel{t \rightarrow \infty}{\longrightarrow} E_{j}
$$

Podemos usar as notações $W_{\delta}^{u}\left(E_{j}\right)$ e $W_{\delta}^{s}\left(E_{j}\right)$ para sermos mais precisos.

Definição 1.48. Dizemos que um invariante isolado $E_{j}$ é uma fonte se

$$
W_{\mathrm{loc}}^{s}\left(E_{j}\right) \cap \mathcal{A}=E_{j},
$$

um sumidouro se

$$
W^{u}\left(E_{j}\right)=E_{j}
$$

e uma sela, caso contrário. 
As definições acima reúnem os conceitos que precisamos para tratar os semigrupos gradientes por meio da teoria a ser apresentada nos Capítulos 2 e 3.

Lema 1.49. Se $(T(\cdot), \Xi)$ é um semigrupo gradiente, então algum $E_{j} \in \Xi$ é um sumidouro e algum $E_{k} \in \Xi$ é uma fonte.

\section{Demonstração:}

Se isto não ocorresse, então para cada $j=1, \ldots, m$, existiriam $x_{j} \in W^{u}\left(E_{j}\right) \backslash E_{j}$ e uma solução $\xi_{j}$ tais que $\xi_{j}(0)=x_{j}$ e $\xi_{j}(t) \stackrel{t \rightarrow-\infty}{\longrightarrow} E_{j}$.

Como $(T(\cdot), \Xi)$ é gradiente, a solução $\xi_{1}$ deve convergir, quando $t \rightarrow \infty$, para algum $E_{j_{1}} \in \Xi$. Da mesma maneira, a solução $\xi_{2}$ é tal que $\xi_{2}(t) \stackrel{t \rightarrow-\infty}{\longrightarrow} E_{j_{2}}$, com $j_{2} \neq j_{1}$, pois não pode haver estrutura homoclínica.

Procedendo assim, como $\Xi$ é finito, somos conduzidos à construção de uma estrutura homoclínica associada a $\Xi$, o que é um absurdo!

A argumentação acima pode ser repetida para verificar que $(T(\cdot), \Xi)$ admite algum invariante isolado fonte: se este não fosse o caso, então existiria uma solução $\xi_{1}$ tal que $\xi_{1}(0)$ pertence à $\delta$-vizinhança de $E_{1}$ e $\xi_{1}(t) \stackrel{t \rightarrow \infty}{\longrightarrow} E_{j_{1}}$, para algum $j_{1} \neq 1$, pois não existem estruturas homoclínicas associadas a $(T(\cdot), \Xi)$. A $E_{j_{1}}$ se aplica o mesmo princípio, ou seja, existe uma $\xi_{j_{2}}$ tal que $\xi_{j_{2}}(0)$ pertence à $\delta$-vizinhança de $E_{j_{2}}$ e $\xi_{j_{2}}(t) \stackrel{t \rightarrow \infty}{\longrightarrow} E_{j_{3}}$, para algum $j_{3} \neq 1, j_{2}$. Uma vez que $\Xi$ é finito, este procedimento nos conduz à construção de uma estrutura homoclínica, o que é um absurdo!

Na Seção 4.1, voltaremos a tratar dos semigrupos gradientes e estas noções locais de atração e repulsão para os invariantes isolados serão recorrentes. 


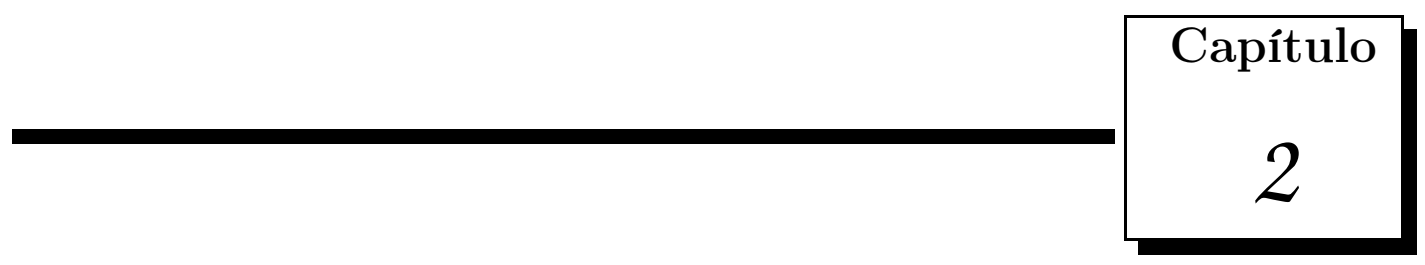

\section{Teoria das Dimensões}

A conjuntos dotados de geometria elementar associamos, de maneira canônica, uma grandeza chamada de dimensão. Pontos têm dimensão 0. Curvas têm dimensão 1. Superfícies têm dimensão 2. Sólidos têm dimensão 3. Porém, quando a geometria elementar é deixada de lado, é fácil encontrar conjuntos que fogem desse seleto grupo e que dificultam essa associação natural; como é o caso dos conjuntos fractais.

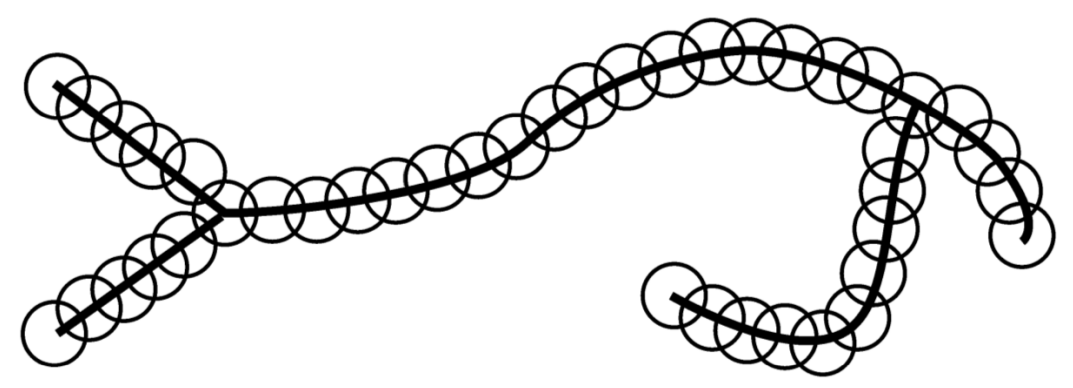

Figura 2.1: Um espaço 1-dimensional e uma cobertura por bolas.

Uma noção geral de dimensão poderia ser fixada para estudar tais conjuntos. Em vez disso, diversas noções foram estabelecidas, cada qual servindo mais apropriadamente a um diferente propósito. Exploramos a dimensão topológica $\left(\operatorname{dim}_{\mathrm{T}}\right)$, pois o principal resultado a ela relacionado, o Teorema da Imersão (Teorema [3.2), serve de inspiração para o resultado que norteia os esforços de unir a Teoria das Dimensões ao estudo de atratores de Sistemas Dinâmicos. Vamos apontar de que forma esta "inspiração" se dá no Teorema de Mañé (Teorema 3.6), no Capítulo 3. Além disso, ela preserva a intuição geométrica de que $\mathbb{R}^{m}$, seus abertos e $m$-variedades têm dimensão $m$. 
Há também as dimensões ditas fractais, por conta de sua forte correlação com os conjuntos fractais. A mais estudada, a mais antiga e provavelmente a mais importante (em termos de aplicabilidade) é a dimensão de Hausdorff, $\operatorname{dim}_{\mathrm{H}}$, definida em espaços métricos. É ainda matematicamente conveniente, uma vez que sua definição está intimamente relacionada a conceitos de medida, diferente da dimensão topológica, cuja essência é de fato topológica. A maior desvantagem, porém, é logo notada: pode ser difícil calculá-la ou mesmo estimá-la por métodos computacionais.

No sentido de favorecer o cômputo e envolver conceitos de manipulação mais simples, uma importante e largamente usada dimensão fractal é a dimensão contagem de caixas superior (livre tradução de (upper) box-counting dimension), a qual é aplicável para espaços métricos compactos. Mas, sua importância é tamanha que a chamaremos simplesmente de dimensão fractal $\left(\operatorname{dim}_{\mathrm{F}}\right)$, sem risco de ambiguidades para o presente texto.

A maneira como estas três dimensões estão relacionadas,

$$
\operatorname{dim}_{\mathrm{T}}(X) \leqslant \operatorname{dim}_{\mathrm{H}}(X) \leqslant \operatorname{dim}_{\mathrm{F}}(X)
$$

faz com que uma herde algumas propriedades da outra e isto certamente será explorado.

Vale apontar algumas variações de nomenclatura e notação:

- dimensão topológica: em inglês, pode ser encontrada sob a denominação de topological dimension, covering dimension, Lebesgue dimension e, às vezes, ČechLebesgue dimension. As notações mais comuns são $\operatorname{dim}_{\mathrm{T}}(X)$ e $\operatorname{cov}(X)$; às vezes, quando não há confusão com o sentido usual de dimensão de espaço vetorial, pode ser denotada por $\operatorname{dim} X$;

- dimensão de Hausdorff: às vezes conhecida como Hausdorff-Besicovitch dimension e com notação variada. Citamos algumas delas: $\operatorname{dim}_{\mathrm{H}}(X), \mathrm{H}-\operatorname{dim}(X)$ e, em alguns textos, $\mathrm{d}_{\mathrm{H}}(X)$ e $\operatorname{HD}(X)$.

- dimensão fractal: encontrada sob os nomes de (upper) box-counting dimension, upper Minkowski-Bouligand dimension, capacidade limite, densidade logarítmica ou entropia de Kolmogorov. Denotada por $\operatorname{dim}_{\mathrm{F}}(X), \overline{\operatorname{dim}_{\mathrm{B}}}(X)$ ou ainda $\overline{\operatorname{dim}_{\mathrm{MB}}}(X)$. 


\subsection{A Dimensão Topológica}

Muito conhecida como covering dimension não à toa, a Figura 2.1 sugere que se um conjunto que merece ser considerado 1-dimensional, então pode ser coberto por pequenas bolas que se interceptam de modo que cada ponto pertença a no máximo 2 destas bolas. Um conjunto é 0-dimensional se pode ser coberto por pequenas bolas disjuntas.

Um conjunto é 2-dimensional se pode ser coberto por pequenas bolas de modo que cada ponto pertença a no máximo 3 destas bolas. Por meio de "blocos" dispostos lado a lado, podemos cobrir o plano de tal forma que cada ponto só pertence a no máximo 3 blocos por vez; e mesmo que expandíssemos tais cubos ligeiramente, ainda poderíamos reposicioná-los de forma que no máximo 3 se interceptam.

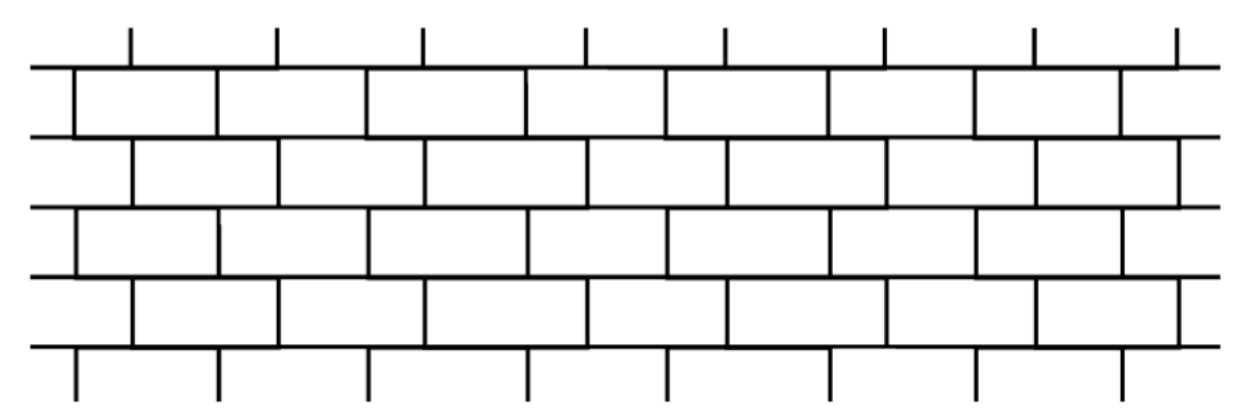

Figura 2.2: Uma cobertura do plano.

A ideia é bastante razoável para espaços métricos compactos, mas requer uma formalização mais precisa se quisermos aplicá-la para espaços métricos não compactos, ou ainda a espaços topológicos quaisquer.

Definição 2.1. Um espaço topológico $X$ tem dimensão topológica finita se existir $m \in \mathbb{Z}_{+}$ tal que toda cobertura aberta $\mathcal{U}$ de $X$ possui um refinamento aberto $\mathcal{U}^{\prime}$ com a propriedade de que cada ponto de $X$ pertence a no máximo $m+1$ abertos de $\mathcal{U}^{\prime}$, i.e., $\mathcal{U}^{\prime}$ é um coleção que tem ordem $m+1$.

Neste caso, a dimensão topológica de X é o menor de tais inteiros não negativos $m$, de modo que é um invariante topológico, o que assina sua essência topológica.

De início, esta definição nos desencoraja a tomá-la como noção padrão de dimensão, uma vez que, dada uma cobertura aberta $\mathcal{U}$ de $X$, investigar todos os possíveis refinamentos abertos de $\mathcal{U}$ à procura de um com ordem $m+1$ é uma tarefa intimidadora e 
certamente não programável, em geral. É mais fácil e também usual encontrar limitantes superiores em vez de valores exatos. E o fato de somente assumir valores naturais nos diz que, em um certo sentido, as particularidades locais de $X$ não são levadas em conta.

Sabidamente, a teoria das dimensões detém seus maiores avanços quando tratada em espaços métricos separáveis. Neste contexto a dimensão topológica admite várias definições alternativas; apresentamos nesta seção 7 delas e outras 2 no Apêndice B. Trabalhar com estas equivalências é como tomar atalhos no que diz respeito a obter resultados para a dimensão topológica.

Quando conhecemos uma nova noção de dimensão, esperamos que ela ao menos coincida com a dimensão usual de espaço vetorial e com os seus objetos "básicos" (pontos em $\mathbb{R}$, retas e figuras planas em $\mathbb{R}^{2}$, retas, superfícies e sólidos em $\mathbb{R}^{3}$, e assim por diante), se for possível calculá-la para tais casos, claro. A dimensão topológica preserva esta intuição, ou seja, $\mathbb{R}^{m}$ tem dimensão topológica $m$, e consequentemente também toda bola aberta $B_{\mathbb{R}^{m}}\left(x_{0}, r\right)$ de $\mathbb{R}^{m}$. É sobre a igualdade $\operatorname{dim}_{\mathrm{T}}\left(\mathbb{R}^{m}\right)=m$ que recai todo o esforço por vir nesta seção. No entanto, verificá-la está longe de ser uma tarefa trivial. Em Munkres [25], o autor se restringe a mostrar que $\mathbb{R}^{m}$ tem dimensão topológica no máximo $m$, reconhecendo a dificuldade de mostrar a igualdade.

A demonstração contida em Engelking [15], talvez uma das mais tradicionais, também recorre ao Teorema do Ponto Fixo de Brouwer e às noções de pequena dimensão indutiva e grande dimensão indutiva, explora a relação entre as três (de fato, coincidem quando $X$ é espaço métrico separável, consulte o Apêndice B ) e obtém a igualdade. O caminho porém se distingue do proposto por Jan Van Mill [21] ao definir a dimensão topológica por meio de coberturas abertas finitas, mesmo sem pedir expressamente que o espaço seja compacto. O Teorema de Compactificação (Engelking [15], Theorem 1.7.2) é a chave para que esta abordagem seja bem sucedida. Segundo ele, todo espaço métrico separável $X$ admite uma compactificação que preserva a dimensão topológica, i.e., existe um espaço métrico compacto $\tilde{X}$ que contém um subespaço denso homeomorfo a $X$ e que satisfaz $\operatorname{dim}_{\mathrm{T}}(\tilde{X})=\operatorname{dim}_{\mathrm{T}}(X)$.

Mais organizada que a abordagem de Engelking [15], seguimos a de Jan Van Mill [21], o qual segue um caminho mais direto e não recorre ao Teorema da Compactificação visto em Engelking [15]. Vamos proceder da seguinte maneira: dar uma definição alternativa para a dimensão topológica em espaços métricos separáveis; mostrar que $\operatorname{dim}_{\mathrm{T}}\left([0,1]^{m}\right)=m$; estabelecer a equivalência entre as definições de dimensão topológica dadas; e finalmente obter que $\operatorname{dim}_{\mathrm{T}}\left(\mathbb{R}^{m}\right)=m$. 
Para esta seção, assume-se que $X$ é um espaço métrico separável. A fim de estabelecer uma noção de dimensão topológica para $X$, vamos precisar de algumas definições.

Definição 2.2. Dados $F, G \subset X$ subconjuntos fechados disjuntos, dizemos que $C \subset X$ é uma partição de $F$ e $G$, ou simplificadamente, que $C$ é uma $(F, G)$-partição, se existem abertos disjuntos $U, V \subset X$ tais que

$$
F \subset U, G \subset V \text { e } X \backslash C=U \cup V
$$

Definição 2.3. Fixado $p \in \mathbb{N}$, dizemos que uma família (finita) de pares de subconjuntos de $X$ fechados disjuntos $\left(F_{j}, G_{j}\right)$, com $j=1, \ldots, p$, é essencial se, para toda familia $\left\{C_{j}: j=1, \ldots, p\right\}$ de $\left(F_{j}, G_{j}\right)$-partições, tem-se

$$
\bigcap_{j=1}^{p} C_{j} \neq \varnothing .
$$

Caso contrário, é dita não essencial.

Os cubos de $\mathbb{R}^{m}$ desempenham papel crucial para mostrar que a dimensão topológica de $\mathbb{R}^{m}$ é exatamente $m$.

Para cada $j=1, \ldots, m$, denotemos por $L_{j}^{(-1)}$ e $L_{j}^{(1)}$ os lados do cubo $[-1,1]^{m}$, ou seja,

$$
L_{j}^{(-1)}=\left\{x \in[-1,1]^{m}: x_{j}=-1\right\} \text { e } L_{j}^{(1)}=\left\{x \in[-1,1]^{m}: x_{j}=1\right\}
$$

de modo que $\left\{\left(L_{j}^{(-1)}, L_{j}^{(1)}\right): j=1, \ldots, m\right\}$ forma uma família de $m$ pares de fechados disjuntos de $[-1,1]^{m}$.

Teorema 2.4. Se $\left\{C_{1}, \ldots, C_{m}\right\}$ é uma família de $m\left(L_{j}^{(-1)}, L_{j}^{(1)}\right)$-partições do cubo $[-1,1]^{m}$, então

$$
\bigcap_{j=1}^{m} C_{j} \neq \varnothing .
$$

Em outras palavras, a família $\left\{\left(L_{j}^{(-1)}, L_{j}^{(1)}\right): j=1, \ldots, m\right\}$ é essencial.

\section{Demonstração:}


Suponha que não, i.e., suponha que

$$
\bigcap_{j=1}^{m} C_{j}=\varnothing
$$

Vamos construir uma função contínua $\xi_{1}:[-1,1]^{m} \rightarrow[-1,1]$ com "boas" propriedades. Como $C_{1}$ é uma $\left(L_{1}^{(-1)}, L_{1}^{(1)}\right)$-partição, existem abertos disjuntos $U_{1}$ e $V_{1}$ tais que

$$
L_{1}^{(-1)} \subset U_{1}, L_{1}^{(1)} \subset V_{1} \text { e }[-1,1]^{m} \backslash C_{1}=U_{1} \cup V_{1},
$$

ou equivalentemente, existem fechados $F_{1}, G_{1} \subset[-1,1]^{m}$ tais que

$$
L_{1}^{(-1)} \subset F_{1}, L_{1}^{(1)} \subset G_{1}, F_{1} \cup G_{1}=[-1,1]^{m} \text { e } F_{1} \cap G_{1}=C_{1},
$$

em que tanto $L_{1}^{(-1)}$ quanto $L_{1}^{(1)}$ são disjuntos de $C_{1}$.

Sendo assim, considere as funções de Urysohn $\alpha, \beta:[-1,1]^{m} \rightarrow[0,1]$ tais que

$$
\left\{\begin{array} { l } 
{ \alpha ^ { - 1 } ( \{ 0 \} ) = C _ { 1 } } \\
{ \alpha ^ { - 1 } ( \{ 1 \} ) = L _ { 1 } ^ { ( - 1 ) } }
\end{array} \text { e } \left\{\begin{array}{l}
\beta^{-1}(\{0\})=C_{1} \\
\beta^{-1}(\{1\})=L_{1}^{(1)}
\end{array}\right.\right.
$$

Defina $\xi_{1}:[-1,1]^{m} \rightarrow[-1,1]$ por

$$
\xi_{1}(x)=\left\{\begin{aligned}
\alpha(x), & \text { se } x \in F_{1} \\
-\beta(x), & \text { se } x \in G_{1}
\end{aligned}\right.
$$

de modo que $\xi_{1}$ é contínua e satisfaz $\xi_{1}\left(L_{1}^{(-1)}\right)=\{1\}, \xi_{1}\left(L_{1}^{(1)}\right)=\{-1\}$ e $\xi_{1}^{-1}(\{0\})=C_{1}$.

Procedendo assim, obtemos funções contínuas $\xi_{j}:[-1,1]^{m} \rightarrow[-1,1]$ tais que

$$
\xi_{j}\left(L_{j}^{(-1)}\right)=\{1\}, \xi_{j}\left(L_{j}^{(1)}\right)=\{-1\} \text { e } \xi_{j}^{-1}(\{0\})=C_{j},
$$

para cada $j=1, \ldots, m$.

Defina então $f:[-1,1]^{m} \rightarrow[-1,1]^{m}$ por

$$
f(x)=\left(\xi_{1}(x), \ldots, \xi_{m}(x)\right)
$$

daí $f$ é contínua e não atinge o ponto $(0,0, \ldots, 0)$, pois $\bigcap_{j=1}^{m} C_{j}=\varnothing$. 
Seja $B$ a fronteira de $[-1,1]^{m}$. Para cada $x \in[-1,1]^{m} \backslash\{0\}$, considere a parametrização da semirreta que parte de $x$ e passa pela origem; e denote por $r(x)$ o ponto no qual tal semirreta atinge $B$.

Uma vez que

$$
[-1,1]^{m} \backslash\{0\} \ni x \longmapsto r(x) \in B
$$

é contínua, a aplicação $g:=r \circ f:[-1,1]^{m} \rightarrow B$ é também contínua e satisfaz

i) $g\left((-1,1)^{m}\right) \cap(-1,1)^{m}=\varnothing$,

ii) $g\left(L_{j}^{(-1)}\right) \subset L_{j}^{(1)}$, para cada $j=1, \ldots, m$, e

iii) $g\left(L_{j}^{(1)}\right) \subset L_{j}^{(-1)}$, para cada $j=1, \ldots, m$.

Portanto, $g$ não admite ponto fixo, o que contradiz o Teorema 1.11 do Ponto Fixo de Brouwer!

Teorema 2.5. Toda família com ao menos $m+1$ pares de subconjuntos fechados disjuntos do cubo unitário $[0,1]^{m} \subset \mathbb{R}^{m}$ é não essencial.

\section{Demonstração:}

Note que toda subfamília de uma família essencial é também essencial, ou equivalentemente, toda família que possui uma subfamília não essencial é também não essencial. Com isso, basta considerarmos o caso em que há $m+1$ pares de fechados disjuntos.

Sejam $F$ e $G$ dois subconjuntos fechados disjuntos de $[0,1]^{m}$, e $D \subset[0,1]$ um subconjunto denso. Primeiramente, verifiquemos que existe uma $(F, G)$-partição $C$ tal que

$$
C \subset\left\{x \in[0,1]^{m}: x_{n} \in D, \text { para algum } n=1, \ldots, m\right\}
$$

De fato, cada ponto $x \in F$ possui uma vizinhança da forma $V_{x}=\left(a_{1}, b_{1}\right) \times \cdots \times$ $\left(a_{m}, b_{m}\right)$, em que $a_{n}, b_{n} \in D$, para todo $n$, tal que $\overline{V_{x}} \cap G=\varnothing$. Da compacidade de $F$, extraímos $p$ vizinhanças $V_{1}, \ldots, V_{p}$ deste tipo de modo que

$$
F \subset \bigcup_{j=1}^{p} V_{j} .
$$


A fronteira $C$ da reunião $\bigcup_{j=1}^{p} V_{j}$ está contida na reunião das fronteiras de $V_{1}, \ldots, V_{p}$, daí $C$ não intercepta $F$ nem $G$ e então é a $(F, G)$-partição procurada.

Agora, dada uma família $\left\{\left(F_{j}, G_{j}\right): j=1, \ldots, m+1\right\}$ de $m+1$ pares de subconjuntos de $[0,1]^{m}$ fechados disjuntos, tome $m+1$ subconjuntos densos de $[0,1]$ dois a dois disjuntos, por exemplo,

$$
D_{1}=(\sqrt{2}+\mathbb{Q}) \cap[0,1], D_{2}=(\sqrt{3}+\mathbb{Q}) \cap[0,1], D_{3}=(\sqrt{5}+\mathbb{Q}) \cap[0,1], \ldots
$$

Pela afirmação acima, existem $\left(F_{j}, G_{j}\right)$-partições $C_{j}$ tais que

$$
C_{j} \subset\left\{x \in[0,1]^{m}: x_{n} \in D_{j} \text {, para algum } n=1, \ldots, m\right\},
$$

para todo $j=1, \ldots, m+1$.

Uma vez que um ponto $x=\left(x_{1}, \ldots, x_{m}\right)$ em $[0,1]^{m}$ tem $m$ coordenadas e $D_{1}, \ldots, D_{m+1}$ são dois a dois disjuntos, não é possível que $x$ pertença a todos os $C_{j}$, ou seja,

$$
\bigcap_{j=1}^{m+1} C_{j}=\varnothing
$$

O Corolário 1.8 diz que $\left\{\left(L_{j}^{(-1)}, L_{j}^{(1)}\right): j=1, \ldots, m\right\}$ admite uma família de partições e o Teorema 2.4 diz ela tem que ser essencial. E por outro lado, o Teorema 2.5 nos diz que toda família de $m+1$ pares de subconjuntos fechados disjuntos do cubo unitário $[0,1]^{m} \subset \mathbb{R}^{m}$ é não essencial. A menos do homeomorfismo entre $[0,1]^{m}$ e $[-1,1]^{m}$, estes resultados nos dizem que estes cubos "ocupam" o mesmo espaço em $\mathbb{R}^{m}$, e esta mensuração está associada ao número $m$.

Com isso, do Teorema 2.5 extraímos uma propriedade topológica de $[0,1]^{m}$ compartilhada com nenhum outro $[0,1]^{n}$ para $n \neq m$. Vale dizer que esta propriedade foi, no início do século XX, uma das grandes responsáveis por instigar matemáticos (especialmente Brouwer, Lebesgue e Poincaré) a pensar em estender apropriadamente as noções usuais de dimensão, dando início ao desenvolvimento da área nas décadas seguintes.

Corolário 2.6. Se $n \neq m$, então $[0,1]^{m}$ não é homeomorfo a $[0,1]^{n}$. 
Estes resultados e observações sugerem uma definição de dimensão, a qual, de fato, é a topológica, no entanto sob outro ponto de vista.

Definição 2.7. Definimos a dimensão topológica de $X$, com a notação $\operatorname{dim}_{\mathrm{T}}(X)$, como sendo

1) $\operatorname{dim}_{\mathrm{T}}(X)=-1$, se $X$ é vazio;

2) $X$ não vazio tem dimensão topológica finita menor ou igual a $m \in \mathbb{Z}_{+}$, indicando por $\operatorname{dim}_{\mathrm{T}}(X) \leqslant m$, quando toda família de $m+1$ pares de subconjuntos fechados disjuntos de $X$ for não essencial;

3) $X$ tem dimensão topológica $m$, indicando por $\operatorname{dim}_{\mathrm{T}}(X)=m$, se $\operatorname{dim}_{\mathrm{T}}(X) \leqslant m$ e $m$ é o menor inteiro positivo que cumpre tal condição;

4) $X$ tem dimensão topológica infinita, e escrevemos $\operatorname{dim}_{\mathrm{T}}(X)=\infty$, se não existe $m$ tal que $\operatorname{dim}_{\mathrm{T}}(X) \leqslant m$.

Seguindo a notação, $\operatorname{dim}_{\mathrm{T}}(X) \geqslant m$ se, e somente se, existe uma família essencial de $m$ pares de fechados disjuntos de $X$. Note que $\operatorname{dim}_{\mathrm{T}}(X) \in\{-1,0,1,2, \ldots\} \cup\{\infty\}$.

Pelos Teoremas 2.4 e 2.5, obtemos

$$
\operatorname{dim}_{\mathrm{T}}\left([0,1]^{m}\right)=m .
$$

E mais geralmente, todos os cubos (fechados) de $\mathbb{R}^{m}$ têm dimensão topológica $m$.

Corolário 2.8. Se $Q \subset \mathbb{R}^{m}$ é um cubo fechado, então $\operatorname{dim}_{\mathrm{T}}(Q)=m$.

Enfatizamos que, a fim de verificar que $\operatorname{dim}_{\mathrm{T}}\left(\mathbb{R}^{m}\right)=m$, a igualdade (2.1.1) é fundamental, bem como o próximo resultado, o qual vai nos custar as próximas páginas para demonstrá-lo.

Teorema 2.9. As definições 2.1 e 2.7 são equivalentes, sempre que $X$ é um espaço métrico separável.

Em primeiro lugar, para facilitar a notação ao longo de toda a seção, associamos a cada coleção de abertos $\mathcal{U}$ de $X$ alguma coleção de índices $\Lambda$, o que é sempre possível, sendo $\mathcal{U}$ enumerável ou não. E assim, escrevemos $\mathcal{U}=\left\{U_{\alpha}\right\}_{\alpha \in \Lambda}$. 
Dizemos que uma tal coleção $\mathcal{U}$ é aberta, se é formada por abertos; que é enumerável, se $\Lambda$ é enumerável; que é finita, se $\Lambda$ é finito; e assim por diante.

Definição 2.10. Sejam $\mathcal{U}=\left\{U_{\alpha}\right\}_{\alpha \in \Lambda}$ e $\mathcal{V}=\left\{V_{\beta}\right\}_{\beta \in \Xi}$ duas coberturas de $X$, dizemos que $\mathcal{V}$ é um refinamento de $\mathcal{U}$ se, para cada $\beta \in \Xi$, existe $\alpha \in \Lambda$ tal que $V_{\beta} \subset U_{\alpha}$.

Define-se um refinamento aberto, inspirado no comentário anterior; e analogamente para refinamento fechado, enumerável ou finito.

Alguns conceitos de Topologia são menos comuns de serem aplicados e, por conta disso, eles carecem de tradução para a Língua Portuguesa, sendo então usados, por mera comodidade, em sua escrita original, na Língua Inglesa.

No entanto, vamos traduzir os termos swelling e shrinking como engrossamento e afinamento, respectivamente. Favor não confundir afinamento com refinamento.

Definição 2.11. Um engrossamento de uma família $\mathcal{U}=\left\{U_{\alpha}\right\}_{\alpha \in \Lambda}$ de subconjuntos de $X$ é uma familia $\mathcal{V}=\left\{V_{\alpha}\right\}_{\alpha \in \Lambda}$ tal que

i) $U_{\alpha} \subset V_{\alpha}$, para cada $\alpha \in \Lambda$; e

ii) para qualquer subcoleção finita de indices $\Lambda^{\prime} \subset \Lambda$, vale

$$
\bigcap_{\alpha \in \Lambda^{\prime}} U_{\alpha}=\varnothing \Leftrightarrow \bigcap_{\alpha \in \Lambda^{\prime}} V_{\alpha}=\varnothing
$$

De certa forma, podemos dizer que um engrossamento de uma família é uma maneira de aumentar os conjuntos um a um, tomando o cuidado de manter conjuntos disjuntos ainda disjuntos.

Proposição 2.12. Toda coleção $\mathcal{F}$ localmente finita de fechados de $X$ admite um engrossamento formado por abertos de $X$.

\section{Demonstração:}

Pela Proposição 1.6, considere $\left\{F_{n}: n \in \mathbb{N}\right\}$ uma enumeração de $\mathcal{F}$, pondo $F_{1}=\varnothing$. Por indução em $n$, vamos construir vizinhanças $U_{n}$ de $F_{n}$ de tal forma que

$$
\mathcal{F}_{n}=\left\{\overline{U_{1}}, \ldots, \overline{U_{n}}, F_{n+1}, F_{n+2}, \ldots\right\}
$$


forma um engrossamento de $\mathcal{F}$.

Seja $U_{1}=\varnothing$ e assuma que, para algum $n$, os conjuntos $U_{1}, \ldots, U_{n}$ foram construídos. Defina $\mathcal{B}$ como sendo a coleção dos conjuntos da forma

$$
E=\bigcap_{j \in J} E_{j}
$$

em que $\left\{E_{j}\right\}_{j \in J}$ é uma subcoleção finita de $\mathcal{F}_{n}$ e tal que $E \cap F_{n+1}=\varnothing$.

Com isso, $\mathcal{B}$ é uma coleção localmente finita de fechados de $X$, de modo que $\mathbf{B}=\bigcup_{E \in \mathcal{B}} E$ é um conjunto fechado de $X$ (Teorema 1.2) tal que $\mathbf{B} \cap F_{n+1}=\varnothing$.

Pelo Corolário 1.8, existe uma vizinhança $U_{n+1}$ de $F_{n+1}$ tal que $\overline{U_{n+1}} \cap \mathbf{B}=\varnothing$. Assim, não é difícil verificar que

$$
\mathcal{F}_{n+1}=\left\{\overline{U_{1}}, \ldots, \overline{U_{n}}, \overline{U_{n+1}}, F_{n+2}, \ldots\right\}
$$

é um engrossamento de $\mathcal{F}$.

Afirmo que $\mathcal{U}=\left\{U_{n}: n \in \mathbb{N}\right\}$ é um engrossamento de $\mathcal{F}$. Claro que $\mathcal{U}$ satisfaz a condição $i$ ) da Definição 2.11. Sejam $\mathbb{N}^{\prime}$ um subconjunto finito de índices em $\mathbb{N}$ tais que

$$
\bigcap_{n \in \mathbb{N}^{\prime}} F_{n}=\varnothing
$$

e $p=\max _{n \in \mathbb{N}^{\prime}} n$. Note que, por construção, $\left\{U_{1}, \ldots, U_{p}\right\}$ é um engrossamento de $\left\{F_{1}, \ldots, F_{p}\right\}$, $\log \mathrm{O}$

$$
\bigcap_{n \in \mathbb{N}^{\prime}} U_{n}=\varnothing
$$

Corolário 2.13. Sejam $\mathcal{F}=\left\{F_{n}: n \in \mathbb{N}\right\}$ uma família localmente finita de fechados de $X$ e $V_{n}$ um aberto tal que $V_{n} \supset F_{n}$, para cada $n$.

Então, $\mathcal{F}$ admite um engrossamento aberto $\mathcal{U}=\left\{U_{n}: n \in \mathbb{N}\right\}$ tal que

$$
\overline{U_{n}} \subset V_{n},
$$

para cada $n \in \mathbb{N}$.

\section{Demonstração:}


Pela Preposição 2.12, tome $\mathcal{W}=\left\{W_{n}: n \in \mathbb{N}\right\}$ um engrossamento aberto de $\mathcal{F}$. Fixado $n$, aplicando o Corolário 1.8 para os fechados disjuntos $F_{n}$ e $X \backslash V_{n}$, obtemos abertos $U_{n}^{(1)}$ e $U_{n}^{(2)}$ tais que

i) $F_{n} \subset U_{n}^{(1)}$,

ii) $X \backslash V_{n} \subset U_{n}^{(2)} \mathrm{e}$

iii) $\overline{U_{n}^{(1)}} \cap \overline{U_{n}^{(2)}}=\varnothing$,

de modo que $\overline{U_{n}^{(1)}} \subset V_{n}$. E procedendo analogamente para os fechados disjuntos $F_{n}$ e $X \backslash W_{n}$, obtemos um aberto $U_{n}^{(3)}$ tal que $F_{n} \subset U_{n}^{(3)} \subset \overline{U_{n}^{(3)}} \subset W_{n}$.

Com isso, defina $U_{n}:=U_{n}^{(1)} \cap U_{n}^{(3)}$ e observe que $U_{n}$ ainda é uma vizinhança de $F_{n}$ e satisfaz

$$
\overline{U_{n}} \subset V_{n} \cap W_{n}
$$

Afirmo que a coleção $\mathcal{U}$ formada por tais abertos $U_{n}$ é o engrossamento procurado.

De fato, se $\mathbb{N}^{\prime}$ é um subconjunto finito de $\mathbb{N}$ tal que

$$
\bigcap_{n \in \mathbb{N}^{\prime}} F_{n}=\varnothing
$$

então

$$
\bigcap_{n \in \mathbb{N}^{\prime}} W_{n}=\varnothing
$$

pois $\mathcal{W}$ é um engrossamento de $\mathcal{F}$; e consequentemente,

$$
\bigcap_{n \in \mathbb{N}^{\prime}} U_{n} \subset \bigcap_{n \in \mathbb{N}^{\prime}}\left(V_{n} \cap W_{n}\right)=\left(\bigcap_{n \in \mathbb{N}^{\prime}} V_{n}\right) \cap\left(\bigcap_{n \in \mathbb{N}^{\prime}} W_{n}\right)=\varnothing .
$$

Definição 2.14. Dadas coberturas quaisquer $\mathcal{U}=\left\{U_{\alpha}\right\}_{\alpha \in \Lambda}$ e $\mathcal{V}=\left\{V_{\alpha}\right\}_{\alpha \in \Lambda}$ de $X$, dizemos que $\mathcal{V}$ é um afinamento de $\mathcal{U}$, quando $V_{\alpha} \subset U_{\alpha}$, para todo $\alpha \in \Lambda$.

Vale notar que todo afinamento é, em particular, um refinamento e que herda a ordem da cobertura original.

Proposição 2.15. Toda cobertura aberta de $X$ admite um afinamento fechado. 


\section{Demonstração:}

Seja $\mathcal{U}$ uma cobertura aberta de $X$, a qual podemos assumir ser enumerável, digamos $\mathcal{U}=\left\{U_{n}: n \in \mathbb{N}\right\}$. Do Corolário [1.8, obtemos, para cada $n$, uma função contínua $f_{n}: X \rightarrow[0,1]$ tal que $f_{n}^{-1}((0,1])=U_{n}$. Defina a função contínua $f: X \rightarrow[0,1]$ por

$$
f(x)=\sum_{n=1}^{\infty} \frac{1}{2^{n}} f_{n}(x)
$$

Dado $x \in X$, como $\mathcal{U}$ cobre $X$, temos $x \in U_{n}$, para algum $n$, daí $f_{n}(x)>0$; com isso, $f(x)>0$, para todo $x \in X$.

Afirmo que os conjuntos fechados

$$
F_{n}=\left\{x \in X: f_{n}(x) \geqslant f(x) / 2\right\} \subset U_{n}
$$

formam uma cobertura de $X$.

Suponha que exista $x \in X$ tal que $x \notin F_{n}$, para todo $n$, daí

$$
f(x)=\sum_{n=1}^{\infty} \frac{1}{2^{n}} f_{n}(x) \leqslant \sum_{n=1}^{\infty} \frac{1}{2^{n}} \frac{f(x)}{2}=\frac{f(x)}{2},
$$

o que contaria o fato de $f(x)>0$.

Corolário 2.16. Se $\operatorname{dim}_{\mathrm{T}}(X) \leqslant m$ e $\mathbb{N}^{\prime}$ é uma subcoleção de $m+2$ indices de $\mathbb{N}$, então toda cobertura aberta enumerável de $X, \mathcal{U}=\left\{U_{n}\right\}_{n \in \mathbb{N}}$, admite um afinamento aberto $\mathcal{V}=\left\{V_{n}\right\}_{n \in \mathbb{N}}$ com as seguintes propriedades:

i) para todo $n \in \mathbb{N}$, tem-se $V_{n} \subset \overline{V_{n}} \subset U_{n} e$

ii) $\bigcap_{n \in \mathbb{N}^{\prime}} V_{n}=\varnothing$

\section{Demonstração:}

Seja $\left\{F_{n}: n \in \mathbb{N}\right\}$ um afinamento fechado de $\mathcal{U}$, conforme a Proposição 2.15, e assuma sem perda de generalidade que $\mathbb{N}^{\prime}=\{1,2, \ldots, m+2\}$. Aplicando o Corolário 1.8 aos fechados disjuntos $F_{n}$ e $X \backslash U_{n}$, para cada $n$ natural, obtemos um aberto $A_{n}$ tal que

$$
F_{n} \subset A_{n} \subset \overline{A_{n}} \subset U_{n}
$$


Como $\operatorname{dim}_{\mathrm{T}}(X) \leqslant m$ e $\left\{\left(X \backslash A_{n}, F_{n}\right): n=1, \ldots, m+1\right\}$ é uma família de $m+1$ pares de conjuntos fechados disjuntos, segue que existem abertos $V_{n}$ e $W_{n}$ tais que $X \backslash A_{n} \subset$ $V_{n}, F_{n} \subset W_{n}, V_{n} \cap W_{n}=\varnothing \mathrm{e}$

$$
\bigcap_{n=1}^{m+1} X \backslash\left(V_{n} \cup W_{n}\right)=\varnothing
$$

ou equivalentemente,

$$
\bigcup_{n=1}^{m+1}\left(V_{n} \cup W_{n}\right)=X
$$

Para cada $n \geqslant m+2$, defina $W_{n}=A_{n} \cap \bigcup_{k=1}^{m+1} V_{k}$, de modo que $\overline{W_{n}} \subset U_{n}$, para tais $n$. Assim,

$$
\begin{aligned}
\bigcup_{n=1}^{\infty} W_{n} & =\left(\bigcup_{n=1}^{m+1} W_{n}\right) \cup\left\{\bigcup_{n=m+2}^{\infty}\left(A_{n} \cap \bigcup_{k=1}^{m+1} V_{k}\right)\right\} \\
& =\left(\bigcup_{n=1}^{m+1} W_{n}\right) \cup\left\{\left(\bigcup_{n=m+2}^{\infty} A_{n}\right) \cap\left(\bigcup_{k=1}^{m+1} V_{k}\right)\right\} \\
& =\left\{\left(\bigcup_{n=1}^{m+1} W_{n}\right) \cup\left(\bigcup_{n=m+2}^{\infty} A_{n}\right)\right\} \cap\left\{\left(\bigcup_{n=1}^{m+1} W_{n}\right) \cup\left(\bigcup_{k=1}^{m+1} V_{k}\right)\right\} \\
& =\left\{\left(\bigcup_{n=1}^{m+1} W_{n}\right) \cup\left(\bigcup_{n=m+2}^{\infty} A_{n}\right)\right\} \cap\left\{\bigcup_{n=1}^{m+1}\left(V_{n} \cup W_{n}\right)\right\} \\
& =\left(\bigcup_{n=1}^{m+1} W_{n}\right) \cup\left(\bigcup_{n=m+2}^{\infty} A_{n}\right) \\
& \supset\left(\bigcup_{n=1}^{m+1} F_{n}\right) \cup\left(\bigcup_{n=m+2}^{\infty} F_{n}\right) \\
& =\bigcup_{n=1}^{\infty} F_{n}=X .
\end{aligned}
$$

Logo, $\mathcal{W}=\left\{W_{n}: n \in \mathbb{N}\right\}$ é um afinamento aberto de $\mathcal{U}$ tal que $\overline{W_{n}} \subset U_{n}$, para todo $n$. 
Além disso,

$$
\begin{aligned}
\bigcap_{n=1}^{m+2} W_{n} & =\left(\bigcap_{n=1}^{m+1} W_{n}\right) \cap\left\{A_{m+2} \cap\left(\bigcup_{k=1}^{m+1} V_{k}\right)\right\} \\
& \subset\left(\bigcap_{n=1}^{m+1} W_{n}\right) \cap\left(\bigcup_{k=1}^{m+1} V_{k}\right) \\
& =\bigcap_{n=1}^{m+1}\left\{W_{n} \cap\left(\bigcup_{k=1}^{m+1} V_{k}\right)\right\} \\
& =\bigcap_{n=1}^{m+1} \bigcup_{k=1}^{m+1}\left(V_{k} \cap W_{n}\right) \\
& =\varnothing
\end{aligned}
$$

pois, caso contrário, encontraríamos $x \in \bigcap_{n=1}^{m+1} \bigcup_{k=1}^{m+1}\left(V_{k} \cap W_{n}\right)$, de modo que, para $n=1$, temos que $x \in V_{k_{1}} \cap W_{1}$, para algum $k_{1} \in\{1, \ldots, m+1\} \backslash\{1\}$.

Para $n=2, x \in V_{k_{2}} \cap W_{2}$, para algum índice $k_{2} \in\{1, \ldots, m+1\} \backslash\{2\}$ e também $k_{2} \neq 1$, novamente porque $V_{1} \cap W_{1}=\varnothing$. Sendo assim, para $n=m$, temos que $x \in V_{k_{m}} \cap W_{m}$, para algum índice $k_{m} \in\{1, \ldots, m+1\} \backslash\{1,2, \ldots, m\}$, i.e., $k_{m}=m+1$. Finalmente, para $n=m+1, x \in V_{k_{m+1}} \cap W_{m+1}$, para algum índice $k_{m+1} \in\{1, \ldots, m+1\} \backslash\{1, \ldots, m+1\}$, o que não é possível!

O resultado abaixo estabelece diversas definições equivalentes à da Definição $2.7 \mathrm{e}, \mathrm{com}$ isso, podemos trabalhar com a noção mais vantajosa nos diferentes resultados relativos à dimensão topológica.

Teorema 2.17. Sejam $X$ um espaço métrico separável não vazio e $m \in \mathbb{Z}_{+}$.

Então, são equivalentes:

a) $\operatorname{dim}_{\mathrm{T}}(X) \leqslant m$;

b) toda cobertura aberta $\mathcal{U}$ de $X$ admite um refinamento fechado localmente finito com ordem $m+1$

c) toda cobertura aberta $\mathcal{U}$ de $X$ admite um refinamento aberto com ordem $m+1$;

d) toda cobertura aberta $\mathcal{U}$ de $X$ admite um afinamento fechado com ordem $m+1$; 
e) toda cobertura aberta $\mathcal{U}$ de $X$ admite um afinamento aberto com ordem $m+1$;

f) toda cobertura aberta finita $\mathcal{U}$ de $X$ admite um afinamento fechado com ordem $m+1$;

g) toda cobertura aberta finita $\mathcal{U}$ de $X$ admite um afinamento aberto com ordem $m+1$.

\section{Demonstração:}

$a) \Rightarrow b)$

Uma vez que $X$ é separável, o Lema 1.5 nos permite assumir que $\mathcal{U}$ é localmente finito; em particular, enumerável:

$$
\mathcal{U}=\left\{U_{0, i}: i \in \mathbb{N}\right\}
$$

A coleção de todos os conjuntos de índices naturais com precisamente $m+2$ índices é enumerável, seja ela $\{I(n): n \in \mathbb{N}\}$. Usando o Corolário 2.16 recursivamente, para cada $n \in \mathbb{N}$, existe um afinamento aberto $\mathcal{V}_{n}=\left\{U_{n, i}: i \in \mathbb{N}\right\}$ de $\mathcal{U}$ tal que

$$
U_{n, i} \subset \bar{U}_{n, i} \subset U_{n-1, i}
$$

para cada natural $i$, e

$$
\bigcap_{i \in I(n)} U_{n, i}=\varnothing
$$

Com isso, afirmo que a coleção $\mathcal{V}=\left\{S_{i}: i \in \mathbb{N}\right\}$, definida por

$$
S_{i}=\bigcap_{n=1}^{\infty} \bar{U}_{n, i}
$$

é uma cobertura de $X$.

Seja $x \in X$. Uma vez que $\mathcal{U}$ é localmente finito, temos que $x$ pertence a uma quantidade finita de abertos de $\mathcal{U}$, de modo que existe $p$ natural tal que $x \notin \bigcup_{i>p} U_{0, i}$. Isto implica que, para cada $n$ natural, existe um índice $k(n) \leqslant p$ tal que $x \in U_{n, k(n)}$, consequentemente, existe $k \leqslant p$ tal que $x$ pertence a uma quantidade infinita de abertos $U_{n, k}$. Pela condição (2.1.2), vemos que $x \in S_{k}$, como queríamos.

Sendo assim, $\mathcal{V}$ é um afinamento fechado de $\mathcal{U}$, consequentemente é também localmente finito; em particular, é um refinamento fechado localmente finito. Além disso, vemos que $\mathcal{V}$ tem ordem $m+1$, por (2.1.2) e (2.1.3). 
$b) \Rightarrow c)$

Por b), existe um refinamento fechado localmente finito $\mathcal{S}$ de $\mathcal{U}$ com ordem $m+1$. Para cada $S \in \mathcal{S}$, tome $U_{S} \in \mathcal{U}$ que contém $S$. Pelo Corolário 2.13, existe um engrossamento aberto $\mathcal{V}=\left\{V_{S}: S \in \mathcal{S}\right\}$ de $\mathcal{S}$ tal que, para cada $S \in \mathcal{S}$, tem-se $V_{S} \subset \overline{V_{S}} \subset U_{S}$.

Assim, como $\mathcal{V}$ herda a ordem de $\mathcal{S}$, segue que $\mathcal{V}$ é um refinamento aberto de $\mathcal{U}$ com ordem $m+1$.

c) $\Rightarrow e)$

Por c), existe um refinamento aberto $\mathcal{W}$ de $\mathcal{U}$ com ordem $m+1$.

Para cada $U \in \mathcal{U}$, defina $V_{U}$ como sendo a reunião dos abertos $W \in \mathcal{W}$ tais que $W \subset U$, de modo que $\mathcal{V}=\left\{V_{U}: U \in \mathcal{U}\right\}$ é um afinamento aberto de $\mathcal{U}$; basta então mostrar que $\mathcal{V}$ tem ordem $m+1$.

Suponha que existam $x \in X$ e abertos $V_{U_{1}}, \ldots, V_{U_{m+2}}$ em $\mathcal{V}$ dois a dois distintos tais que

$$
x \in \bigcap_{j=1}^{m+2} V_{U_{j}}
$$

de modo que, para cada $j=1, \ldots, m+2$, existe $W_{j} \in \mathcal{W}$ tal que $x \in W_{j} \subset U_{j}$, pela construção de $V_{U_{j}}$.

Note que, sendo $V_{U_{1}}, \ldots, V_{U_{m+2}}$ dois a dois distintos, tem-se $U_{1}, \ldots, U_{m+2}$ dois a dois distintos e consequentemente, $W_{1}, \ldots, W_{m+2}$ são dois a dois distintos.

Como $\mathcal{W}$ tem ordem $m+1$, temos que $x \in \bigcap_{j=1}^{m+2} W_{j}=\varnothing$, o que é um absurdo. Sendo assim, $\mathcal{V}$ é um afinamento aberto de $\mathcal{U}$ com ordem $m+1$.

$e) \Rightarrow d)$

Como o afinamento de uma cobertura de $X$ herda a ordem desta cobertura, o item $d$ ) segue de $e$ ) e da Proposição 2.15.

$d) \Rightarrow f)$

Imediato!

$f) \Rightarrow g)$

Segue como consequência do Corolário 2.13 ,

$g) \Rightarrow a)$ 
Seja $\left\{\left(A_{j}, B_{j}\right): j=1, \ldots, m+1\right\}$ uma família de $m+1$ pares de fechados disjuntos de $X$, de modo que

$$
\left\{X \backslash A_{1}, X \backslash A_{2}, \ldots, X \backslash A_{m+1}, X \backslash \bigcup_{j=1}^{m+1} B_{j}\right\}
$$

é uma cobertura de $X$, pois se $x$ não pertence a $X \backslash \bigcup B_{j}$, então $x \in B_{j}$, para algum $j$, daí $x \in X \backslash A_{j}$. Por $g$ ), tal cobertura admite um afinamento aberto $\mathcal{V}=\left\{V_{j}: j=1, \ldots, m+2\right\}$ de ordem $m+1$, em que

$$
V_{j} \subset\left(X \backslash A_{j}\right)
$$

para cada $j=1, \ldots, m+1$, e

$$
V_{m+2} \subset X \backslash \bigcup_{j=1}^{m+1} B_{j}
$$

Pela Proposição 2.15, $\mathcal{V}$ admite um afinamento fechado $\left\{F_{j}: j=1, \ldots, m+2\right\}$. Agora, para cada $j=1, \ldots, m+2$, defina

$$
\mathbf{A}_{j}=\left(F_{m+2} \backslash V_{j}\right) \cup A_{j}
$$

e

$$
\mathbf{B}_{j}=F_{j} \cup B_{j},
$$

de modo que $\mathbf{A}_{j} \cap \mathbf{B}_{j}=\varnothing$, pois $F_{j} \subset V_{j} \subset\left(X \backslash A_{j}\right)$ e $F_{m+2} \cap B_{j}=\varnothing$.

Claro que

$$
\bigcup_{j=1}^{m+1} F_{j} \subset \bigcup_{j=1}^{m+1} \mathbf{B}_{j}
$$

e por outro lado, afirmo que

$$
F_{m+2} \subset \bigcup_{j=1}^{m+1} \mathbf{A}_{j}
$$

De fato, sabemos que $F_{m+2} \subset V_{m+2}$ e que $V_{m+2} \cap \bigcap_{j=1}^{m+1} V_{j}=\varnothing$, pois $\mathcal{V}$ ter ordem $m+1$. Com isso,

$$
F_{m+2}=F_{m+2} \backslash\left(\bigcap_{j=1}^{m+1} V_{j}\right) \subset \bigcup_{j=1}^{m+1} \mathbf{A}_{j} .
$$

Pelo Corolário 1.8, para cada $j=1, \ldots, m+1$, existe uma $\left(\mathbf{A}_{j}, \mathbf{B}_{j}\right)$-partição $L_{j}$, a 
qual é uma $\left(A_{j}, B_{j}\right)$-partição, pois $A_{j} \subset \mathbf{A}_{j}$ e $B_{j} \subset \mathbf{B}_{j}$. Logo,

$$
\bigcap_{j=1}^{m+1} L_{j} \subset X \backslash\left(\bigcup_{j=1}^{m+1}\left(\mathbf{A}_{j} \cup \mathbf{B}_{j}\right)\right) \subset X \backslash\left(F_{m+2} \cup \bigcup_{j=1}^{m+1} F_{j}\right)=X \backslash X=\varnothing
$$

Ou seja, a família $\left\{\left(A_{j}, B_{j}\right): j=1, \ldots, m+1\right\}$ de pares de fechados disjuntos de $X$ admite uma família de $\left(A_{j}, B_{j}\right)$-partições $\left\{L_{j}: j=1, \ldots, m+1\right\}$ tal que

$$
\bigcap_{j=1}^{m+1} L_{j}=\varnothing
$$

o que mostra que $\operatorname{dim}_{\mathrm{T}}(X) \leqslant m$, por definição.

O Teorema 2.17 é de grande importância, pois nos permite trabalhar com a definição de dimensão topológica que mais for conveniente, assumindo que é possível saber qual é mais conveniente. Dele também segue o Teorema 2.9, segundo o qual as Definições 2.1 e 2.7 de dimensão topológica são equivalentes, quando $X$ é um espaço métrico separável.

Vale apontar que o nosso interesse frequentemente recai sobre espaços métricos compactos - o atrator global $\mathcal{A}$ de um semigrupo -, de modo que, para os propósitos deste texto, a dimensão topológica pode sempre ser entendida como na Definição 2.7.

A seguir, temos um importante resultado que relaciona de que forma subconjuntos fechados de $X$ podem determinar a dimensão topológica do espaço. É conhecido como o Teorema da Soma Enumerável de Fechados.

Teorema 2.18. Seja $\mathcal{F}=\left\{F_{j}: j \in \mathbb{N}\right\}$ uma cobertura enumerável fechada de $X$ tal que $\operatorname{dim}_{\mathrm{T}}\left(F_{j}\right) \leqslant m$, para todo $j \in \mathbb{N}$.

Então, $\operatorname{dim}_{\mathrm{T}}(X) \leqslant m$.

\section{Demonstração:}

Assuma $m<\infty$ e adicione a $\mathcal{F}$ o conjunto $F_{0}:=\varnothing$.

Vamos usar o item $f$ ) do Teorema 2.17 para mostrar que $\operatorname{dim}_{\mathrm{T}}(X) \leqslant m$.

Seja $\mathcal{U}$ uma cobertura aberta finita de $X$. Por indução em $j \geqslant 0$, podemos construir uma cobertura aberta $\mathcal{U}(j)$ de $X$ de tal forma que

i) $\mathcal{U}(0)=\mathcal{U}$ 
ii) se $0 \leqslant i<j$, então $\overline{\mathcal{U}(j)}$ é um afinamento de $\mathcal{U}(i)$,

iii) a ordem de $\overline{\mathcal{U}(j)} \cap F_{j}$ é $m+1$.

Para isso, em cada passo $j$, basta usar a Proposição 2.15 para obter um afinamento fechado de $\mathcal{U}(j-1)$, então o Corolário 2.13 para obter um engrossamento aberto (que satisfaça ii) ) e, como $\operatorname{dim}_{\mathrm{T}}\left(F_{j}\right) \leqslant m$, usar o item $g$ ) do Teorema 2.17 para obter um afinamento aberto de ordem $m+1$.

Seja $k$ a cardinalidade de $\mathcal{U}$. Claro que, para cada $j \in \mathbb{N}$, podemos enumerar $\mathcal{U}(j)$ como $\left\{U_{n, j}: n=1, \ldots, k\right\}$ tal que $0 \leqslant j<i$ e $n \leqslant k$ implicam $\bar{U}_{n, i} \subset U_{n, j}$. Agora para cada $x \in X$ existe $n(x) \leqslant k$ tal que $x$ pertence a infinitos conjuntos $U_{n(x), j}$. Então, por construção, temos que $x \in \bigcap_{j=1}^{\infty} U_{n(x), j}$, de modo que a coleção dos conjuntos $\bigcap_{j=1}^{\infty} U_{n, j}$, com $n=1, \ldots, k$, forma uma cobertura para $X$.

Por ii) e iii), concluímos que

$$
\left\{\bigcap_{j=1}^{\infty} U_{n, j}: n=1, \ldots, k\right\}=\left\{\bigcap_{j=1}^{\infty} \bar{U}_{n, j}: n=1, \ldots, k\right\}
$$

é um afinamento fechado de $\mathcal{U}$ e tem ordem $m+1$.

Consequentemente, $\operatorname{dim}_{\mathrm{T}}(X) \leqslant m$.

Basta reunir resultados e observações já feitas para concluir que

$$
\operatorname{dim}_{\mathrm{T}}\left(\mathbb{R}^{m}\right) \leqslant m
$$

De fato, como podemos escrever

$$
(0,1)^{m}=\bigcup_{n=1}^{\infty}[1 / n, 1-1 / n]^{m}
$$

e cada cubo $[1 / n, 1-1 / n]^{m}$ tem dimensão topológica igual a $m$, segue do Teorema 2.18 que

$$
\operatorname{dim}_{\mathrm{T}}\left(\mathbb{R}^{m}\right)=\operatorname{dim}_{\mathrm{T}}\left((0,1)^{m}\right) \leqslant m .
$$

Ou alternativamente, o Teorema do Subespaço (Teorema 2.19 abaixo) nos diz que a dimensão topológica do espaço domina a dimensão topológica de seus subespaços. Uma 
vez que $(0,1)^{m}$ é subespaço de $[0,1]^{m}$, segue que $\operatorname{dim}_{\mathrm{T}}\left((0,1)^{m}\right) \leqslant m$, o que novamente implica a desigualdade (2.1.4).

Teorema 2.19. Se $Y$ é um subespaço de $X$, então $\operatorname{dim}_{\mathrm{T}}(Y) \leqslant \operatorname{dim}_{\mathrm{T}}(X)$.

\section{Demonstração:}

Como não há o que provar se $\operatorname{dim}_{\mathrm{T}}(X)=\infty$, suponha que seja finita e igual a $m$.

De início, assuma que $Y$ é fechado. Uma vez que toda família essencial em $Y$ é também uma família essencial em $X$, pois a definição envolve conjuntos fechados em $Y$, segue que $\operatorname{dim}_{\mathrm{T}}(Y) \leqslant m$

Agora, suponha que $Y$ é aberto, e note que podemos escrever

$$
Y=\bigcup_{n=1}^{\infty} Y_{n},
$$

em que cada $Y_{n}=\{x \in Y: d(x, X \backslash Y) \geqslant 1 / n\}$ é um fechado de $X, \operatorname{logo} \operatorname{dim}_{\mathrm{T}}\left(Y_{n}\right) \leqslant m$ e então pelo Teorema 2.18, segue que $\operatorname{dim}_{\mathrm{T}}(Y) \leqslant m$.

Finalmente, sejam $Y$ um subespaço qualquer de $X$ e $\mathcal{U}=\left\{U_{\alpha} \cap Y\right\}_{\alpha \in \Lambda}$ uma cobertura de $Y$ por abertos em $Y$, em que cada $U_{\alpha}$ é um aberto de $X$.

Seja

$$
U=\bigcup_{\alpha \in \Lambda} U_{\alpha}
$$

de modo que $U$ é um aberto de $X$; consequentemente, $\operatorname{dim}_{\mathrm{T}}(U) \leqslant m$.

Claro que a família $\left\{U_{\alpha}: \alpha \in \Lambda\right\}$ é uma cobertura aberta de $U$, daí pelo Teorema 2.17 aplicado a $U$, tal cobertura admite um refinamento aberto $\mathcal{V}$ que tem ordem $m+1$. A partir daí, vemos que $\mathcal{W}=\{V \cap Y: V \in \mathcal{V}\}$ é um refinamento aberto de $\mathcal{U}$ que tem ordem $m+1$. Novamente, pelo Teorema 2.17, concluímos que $\operatorname{dim}_{\mathrm{T}}(Y) \leqslant m$, como queríamos.

Com o Teorema 2.19 a nossa disposição, é possível encontrar vários espaços zero dimensionais e apresentar uma terceira demonstração de que $\operatorname{dim}_{\mathrm{T}}\left(\mathbb{R}^{m}\right) \leqslant m$.

Para cada $0 \leqslant k \leqslant m$, defina

$$
\mathbb{R}_{k}^{m}=\left\{x \in \mathbb{R}^{m}: x \text { possui exatamente } k \text { coordenadas racionais }\right\}
$$

e note que

$$
\mathbb{R}^{m}=\mathbb{R}_{0}^{m} \cup \mathbb{R}_{1}^{m} \cup \cdots \cup \mathbb{R}_{m}^{m}
$$


É possível mostrar que $\operatorname{dim}_{\mathrm{T}}\left(\mathbb{R}_{k}^{m}\right)=0$ sem grande esforço (consulte Jan van Mill [21]). Sabemos ainda que se um espaço métrico separável $X$ pode ser escrito como reunião de até $m+1$ subespaços de dimensão topológica zero, então $\operatorname{dim}_{\mathrm{T}}(X) \leqslant m$ (Apêndice $\mathrm{A}$, Teorema A.6, item 6). Com isso,

$$
\operatorname{dim}_{\mathrm{T}}\left(\mathbb{R}^{m}\right) \leqslant m
$$

Finalmente, chegamos ao resultado que norteou esta seção; às vezes, conhecido como o Teorema Fundamental da Teoria da Dimensão.

Teorema 2.20. Para todo $m$, vale $\operatorname{dim}_{\mathrm{T}}\left(\mathbb{R}^{m}\right)=m$.

\section{Demonstração:}

Sabemos que $\operatorname{dim}_{\mathrm{T}}\left(\mathbb{R}^{m}\right) \leqslant m$.

E pelos Teoremas 2.8 e 2.19, temos

$$
m=\operatorname{dim}_{\mathrm{T}}\left([0,1]^{m}\right) \leqslant \operatorname{dim}_{\mathrm{T}}\left(\mathbb{R}^{m}\right)
$$

Duas consequências imediatas do Teorema 2.20 são que

$$
\operatorname{dim}_{\mathrm{T}}(U)=m,
$$

para todo $U \subset \mathbb{R}^{m}$ aberto, e que $\mathbb{R}^{m}$ não é homeomorfo a $\mathbb{R}^{n}$, se $n \neq m$.

\subsection{A Dimensão de Hausdorff}

Em diversos problemas geométricos, é importante ter um método de medir o tamanho de conjuntos de menor dimensão em $\mathbb{R}^{m}$, tais como curvas e surperfícies. Questões sobre o quanto medem conjuntos enumeráveis, intervalos e superfícies $m$-dimensionais serão levantadas e respondidas. No entanto, o intuito é fornecer familiaridade aos conceitos e alguns resultados relativos à medida e à dimensão de Hausdorff, para aplicá-los ao contexto de semigrupos. Para mais informações, consulte Edgar [12], Falconer [16], Falconer [17], Folland [18] e Pesin [26]. 
A dimensão de Hausdorff é uma noção de dimensão para espaços métricos $(X, \mathrm{~d})$ que, diferente das usuais, está intimamente relacionada à métrica d e a uma medida no espaço, a medida de Hausdorff. Enquanto a dimensão topológica assume valores em $\{-1,0,1,2, \ldots\} \cup\{\infty\}$, a de Hausdorff toma valores em $[0, \infty]$, muito mais abrangente. Esta diferença será sentida quando vermos que a dimensão de Hausdorff é capaz de mensurar conjuntos que, sob a perspectiva da dimensão topológica, seriam zero dimensionais, como é o caso do conjunto de Cantor em $\mathbb{R}$.

Ainda que não seja o foco deste trabalho, vale apontar que as dimensões topológica e de Hausdorff têm relação muito íntima com os conjuntos fractais. De fato, para muitos pesquisadores, um conjunto fractal $E \subset X$ é aquele tal que $\operatorname{dim}_{\mathrm{T}}(E)<\operatorname{dim}_{\mathrm{H}}(E)$, mas não existe consenso quanto a esta definição, até porque importantes conjuntos fractais não satisfazem essa relação. Tome como exemplo o conjunto de Smith-Volterra-Cantor $C \subset \mathbb{R}$, a fronteira do conjunto de Mandelbrot, $M \subset \mathbb{R}^{2}$, e a curva de Hilbert $3 \mathrm{D}, H \subset \mathbb{R}^{3}$, os quais têm dimensão de Hausdorff, respectivamente, 1,2 e 3.

Dizemos que $\left\{A_{j}\right\}_{j}$ é uma $\delta$-cobertura de $A$ se for uma coleção enumerável de subconjuntos de $X$ tal que $A \subset \bigcup_{j=1}^{\infty} A_{j}$ e $\operatorname{diam}\left(A_{j}\right)<\delta$ para todo $j \in \mathbb{N}$. As coberturas que vamos considerar serão todas enumeráveis e a razão está implícita na definição de $\mu_{\delta}^{(\alpha)}(A)$ por vir: para medir $A$, associamos a cada $A_{j}$ um certo peso (positivo), $\operatorname{diam}\left(A_{j}\right)^{\alpha}$, e então somamos em $j$, o índice da cobertura. E sabidamente, coleções não enumeráveis de números positivos não podem ter soma finita. Vale ainda apontar que um tal conjunto $A$, munido da métrica induzida de $X$, admite uma $\delta$-cobertura, para qualquer $\delta$ dado, se e somente se, $A$ é separável.

Queremos usar o número

$$
\sum_{j=1}^{\infty}\left(\operatorname{diam}\left(A_{j}\right)\right)^{\alpha}
$$

às vezes dito $\alpha$-potencial associado à $\delta$-cobertura $\left\{A_{j}\right\}_{j}$, para medir quão "grande" o conjunto $A$ é, em algum sentido.

Dados $\alpha \geqslant 0$ e $\delta>0$, definimos para $A \subset X$,

$$
\mu_{\delta}^{(\alpha)}(A)=\inf \left\{\sum_{j=1}^{\infty}\left(\operatorname{diam}\left(A_{j}\right)\right)^{\alpha}: A \subset \bigcup_{j=1}^{\infty} A_{j}, \operatorname{diam}\left(A_{j}\right)<\delta\right\}
$$

com a convenção de que inf $\varnothing=\infty$. A semelhança com a definição de medida exterior 
de Lebesgue em $\mathbb{R}^{m}$, denotada por $\mathcal{L}^{m}$, não é ao acaso, como veremos. Lembrando que, dado $A \subset \mathbb{R}^{m}$,

$$
\mathcal{L}^{m}(A)=\inf \left\{\sum_{j=1}^{\infty} V\left(Q_{j}\right): A \subset \bigcup_{j=1}^{\infty} Q_{j}\right\}
$$

em que $Q_{j}$ são cubos semiabertos e $V\left(Q_{j}\right)$ denota seu volume usual.

Vamos adotar como convenção que

$$
(\operatorname{diam}(E))^{0}=\left\{\begin{array}{ll}
0, & \text { se } E=\emptyset \\
1, & \text { caso contrário }
\end{array},\right.
$$

para um subconjunto $E \subset X$ qualquer, e disso obter que

- $\mu^{(0)}(E)=0$, se $E=\emptyset$;

- $\mu^{(0)}(E)=n$, se $E$ tem $n$ elementos;

- $\mu^{(0)}(E)=\infty$, se $E$ tem infinitos elementos.

Sejam $\delta_{1}>\delta_{2}>0$. Uma vez que toda $\delta_{2}$-cobertura é também uma $\delta_{1}$-cobertura, segue que $\mu_{\delta_{1}}^{(\alpha)}(A) \leqslant \mu_{\delta_{2}}^{(\alpha)}(A)$, de modo que $\delta \mapsto \mu_{\delta}^{(\alpha)}(A)$ é monótona não crescente. Assim, definimos a $\alpha$-medida de Hausdorff de $A$ por

$$
\mu^{(\alpha)}(A)=\lim _{\delta \rightarrow 0^{+}} \mu_{\delta}^{(\alpha)}(A)
$$

a qual pode valer $\infty$; de fato isto ocorre com frequência.

Acontece que $\mu^{(\alpha)}: \mathcal{P}(X) \rightarrow[0, \infty]$ é uma medida exterior métrica e também o ingrediente principal para definirmos a dimensão de Hausdorff e provar algumas de suas propriedades.

Das definições de limite e ínfimo, segue que se $\mu^{(\alpha)}(A)<\infty$ então, dados $\varepsilon, \delta>0$, existe uma $\delta$-cobertura $\left\{A_{j}\right\}_{j}$ de $A$ tal que

$$
\left|\mu^{(\alpha)}(A)-\sum_{j=1}^{\infty}\left(\operatorname{diam}\left(A_{j}\right)\right)^{\alpha}\right| \leqslant \varepsilon .
$$

Se considerássemos $\delta$-coberturas fechadas em vez de $\delta$-coberturas quaisquer, obteríamos uma definição equivalente de $\alpha$-medida de Hausdorff, pois para todo $A \subset X$, tem-se $\operatorname{diam}(A)=\operatorname{diam}(\bar{A})$. Similarmente, pode ser conveniente nos restringirmos a $\delta$-coberturas 
abertas, observando que estas, para cada $\delta$, podem fornecer diferentes valores de $\mu_{\delta}^{(\alpha)}(A)$ mas o limite $\mu^{(\alpha)}(A)$ é o mesmo. Consulte Falconer [17].

Denotemos por $\nu_{\delta}^{(\alpha)}(A)$ o ínfimo dos números $\sum_{j=1}^{\infty}\left(\operatorname{diam}\left(V_{j}\right)\right)^{\alpha}$ tomado sobre todas as $\delta$-coberturas abertas $\left\{V_{j}\right\}_{j}$ de $A$; e seja $\nu^{(\alpha)}(A)=\lim _{\delta \rightarrow 0^{+}} \nu_{\delta}^{(\alpha)}(A)$.

Claro que $\mu_{\delta}^{(\alpha)}(A) \leqslant \nu_{\delta}^{(\alpha)}(A)$. Por outro lado, suponha $\mu_{\delta}^{(\alpha)}(A)<\infty$. Dado $\varepsilon>0$, para cada $\delta>0$, existe uma $\delta$-cobertura $\left\{A_{j}\right\}_{j}$ de $A$ tal que $\left|\mu^{(\alpha)}(A)-\sum\left(\operatorname{diam}\left(A_{j}\right)\right)^{\alpha}\right| \leqslant \varepsilon$.

Para cada $j$, seja $V_{j}=\mathcal{O}_{r_{j}}\left(A_{j}\right)$, em que $r_{j}>0$ é suficientemente pequeno de tal forma que $\operatorname{diam}\left(V_{j}\right)<\delta$ e $\left(\operatorname{diam}\left(V_{j}\right)\right)^{\alpha} \leqslant\left(\operatorname{diam}\left(A_{j}\right)\right)^{\alpha}+\varepsilon / 2^{j}$, daí

$$
\nu_{\delta}^{(\alpha)}(A) \leqslant \sum_{j=1}^{\infty}\left(\operatorname{diam}\left(V_{j}\right)\right)^{\alpha} \leqslant \sum_{j=1}^{\infty}\left(\operatorname{diam}\left(A_{j}\right)\right)^{\alpha}+\varepsilon \leqslant \mu^{(\alpha)}(A)+2 \varepsilon
$$

$\log 0 \nu_{\delta}^{(\alpha)}(A) \leqslant \mu^{(\alpha)}(A)+2 \varepsilon$, para todo $\varepsilon>0$ e todo $\delta>0$, de onde obtemos $\nu_{\delta}^{(\alpha)}(A) \leqslant$ $\mu_{\delta}^{(\alpha)}(A)$.

Até agora, o parâmetro $\alpha$ tem aparecido mas mantido constante. Mas é sobre ele que recai o estágio final da definição de dimensão de Hausdorff.

Há três possibilidades para $\mu^{(\alpha)}(A)$ : ser $0, \infty$ ou finito maior que 0 . Esta última é a de maior interesse e traz drásticas consequências, como o resultado a seguir, enquanto que as duas primeiras possibilidades pouco se relacionam com as noções de cardinalidade, comprimento e área de que falamos.

Tentemos esboçar o gráfico da função $\alpha \mapsto \mu^{(\alpha)}(A)$ :

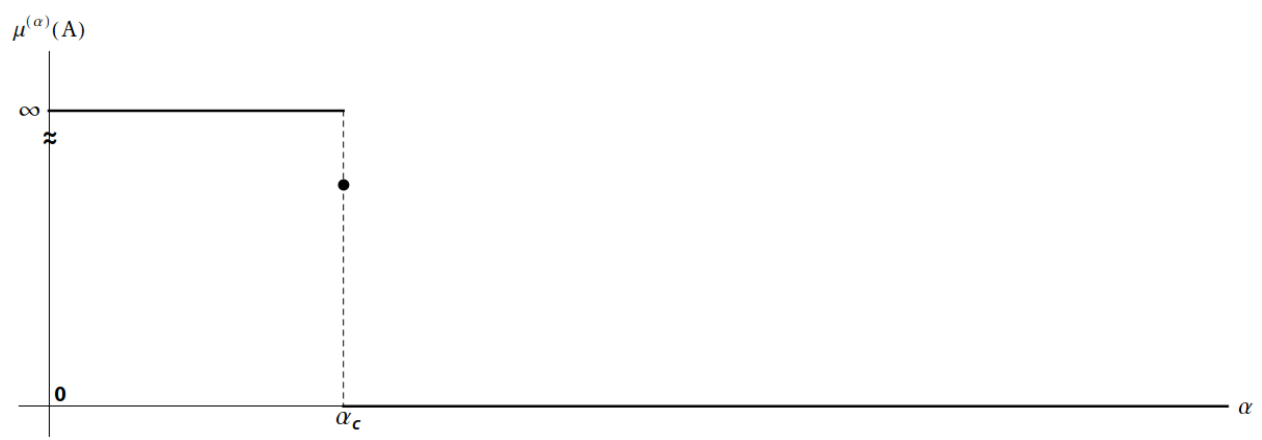

Figura 2.3: O valor crítico $\alpha_{c}$ é a dimensão de Hausdorff. 
Proposição 2.21. Se $\alpha \geqslant 0$ é tal que $\mu^{(\alpha)}(A)<\infty$, então

$$
\mu^{(\alpha+\varepsilon)}(A)=0
$$

para todo $\varepsilon>0$, ou equivalentemente, se $\mu^{(\alpha+\varepsilon)}(A)>0$, então $\mu^{(\alpha)}(A)=\infty$.

Demonstração: Se $\mu^{(\alpha)}(A)<\infty$, então, para cada $\delta>0$, obtemos uma $\delta$-cobertura $\left\{A_{j}\right\}_{j}$ tal que

$$
\sum_{j=1}^{\infty}\left(\operatorname{diam}\left(A_{j}\right)\right)^{\alpha} \leqslant \mu^{(\alpha)}(A)+1 .
$$

Dado $\varepsilon>0$, temos

$$
\begin{aligned}
& \sum_{j=1}^{\infty}\left(\operatorname{diam}\left(A_{j}\right)\right)^{\alpha+\varepsilon}<\delta^{\varepsilon} \sum_{j=1}^{\infty}\left(\operatorname{diam}\left(A_{j}\right)\right)^{\alpha} \leqslant \delta^{\varepsilon}\left(\mu^{(\alpha)}(A)+1\right) \\
\Rightarrow & \mu_{\delta}^{(\alpha+\varepsilon)}(A) \leqslant \delta^{\varepsilon}\left(\mu^{(\alpha)}(A)+1\right) \stackrel{\delta \rightarrow 0^{+}}{\longrightarrow} 0 \\
\Rightarrow & \mu^{(\alpha+\varepsilon)}(A)=0 .
\end{aligned}
$$

Com isso, justificamos a Figura 2.3 e concluímos que $\alpha \mapsto \mu^{(\alpha)}(A)$ fica inteiramente determinada pelo valor crítico $\alpha_{c}$ e por $\mu^{\left(\alpha_{c}\right)}(A) \in[0, \infty]$, sendo $\alpha_{c}$ o ponto no qual a função "passa" de $\infty$ para 0 . Esta quantidade $\mu^{\left(\alpha_{c}\right)}(A)$ obviamente depende de $A$ e este é o ponto!

A dimensão de Hausdorff de $A$, indicada por $\operatorname{dim}_{\mathrm{H}}(A)$ ou $\operatorname{simplesmente} \operatorname{dim}_{\mathrm{H}} A$, é então o valor crítico $\alpha_{c}$ associado a $A$, ou seja,

$$
\operatorname{dim}_{\mathrm{H}}(A):=\inf \left\{\alpha \geqslant 0: \mu^{(\alpha)}(A)=0\right\}
$$

ou alternativamente,

$$
\operatorname{dim}_{\mathrm{H}}(A)=\sup \left\{\alpha \geqslant 0: \mu^{(\alpha)}(A)=\infty\right\}
$$

Quando $\mu^{(\alpha)}(A) \neq 0$, para todo $\alpha \geqslant 0$, definimos que $\operatorname{dim}_{\mathrm{H}}(A)=\infty$.

Vale observar que se $\alpha_{0} \geqslant 0$ é tal $0<\mu^{\left(\alpha_{0}\right)}(A)<\infty$, então $\operatorname{dim}_{\mathrm{H}}(A)=\alpha_{0}$. 
Agora que temos a definição, vale perguntar em que sentido isto é uma noção de "dimensão" e se concorda com o que entendemos intuitivamente por dimensão. E mais, devemos investigar que propriedades satisfaz e que relação pode ter com a usual medida de Lebesgue, especialmente em espaços euclidianos finito dimensionais.

Nesta direção, primeiramente observemos que um ponto de $X$ tem $\alpha$-medida de Hausdorff zero, qualquer que seja $\alpha \geqslant 0$ : para um dado $x \in X$, considere a cobertura enumerável $\{x\}$, a qual é uma $\delta$-cobertura de $\{x\}$, para todo $\delta>0$, pois diam $(\{x\})=0$, e então $\mu^{(\alpha)}(x)=0$.

Logo veremos que a $\alpha$-medida de Hausdorff é uma medida exterior métrica e, consequentemente, os conjuntos enumeráveis têm $\alpha$-medida de Hausdorff zero.

Ganharemos mais intuição quando provarmos que a dimensão de Hausdorff de um intervalo $I$ real é igual a 1 . Mais precisamente, que a 1-medida de Hausdorff de $I$ é igual ao seu comprimento.

Antes disso, consideremos a medida de Lebesgue de $\mathbb{R}, m$, e um tradicional boreliano, o conjunto de Cantor dos terços médios, $C \subset[0,1]$. Logo percebemos que $C$ é muito mais que um ponto ou um conjunto enumerável, os quais têm medida de Lebesgue nula, mas muito menos que o intervalo $[0,1]$, o qual tem medida de Lebesgue unitária. De fato, $C$ é um caso intermediário mas cujo "tamanho" não é bem percebido pela medida de Lebesgue, já que $m(C)=0$. No entanto, $\operatorname{dim}_{H}(C)=\log 2 / \log 3$; mais precisamente, pondo $\alpha=\log 2 / \log 3$, temos que $\mu^{(\alpha)}(C)=1$, ou seja, esta $\alpha$-medida de Hausdorff enxerga a diferença entre conjuntos enumeráveis $E$, o conjunto de Cantor $C$ e o intervalo $[0,1]$ :

$$
\mu^{(\alpha)}(E)=0 \leqslant \mu^{(\alpha)}(C)=1<\mu^{(\alpha)}([0,1])=\infty
$$

Para mostrar que $\mu^{(\alpha)}(C) \leqslant 1$, basta considerar, para cada $k$, os $2^{k}$ intervalos disjuntos de comprimento $1 / 3^{k}$ que surgem na construção de $C$. Agora, dada uma cobertura enumerável $\left\{A_{j}\right\}_{j}$ aberta de $C$, a outra desigualdade segue da compacidade de $C$ e de uma decomposição apropriada de cada aberto $A_{j}$. Consulte Falconer [17]. Verificar que $\mu^{(\alpha)}(C) \geqslant 1 / 2$ é uma tarefa surpreendentemente mais simples que verificar que $\mu^{(\alpha)}(C) \geqslant 1$. Esta observação aponta para um problema prático ao lidar com a dimensão de Hausdorff: é consideravelmente mais fácil encontrar que uma "boa" cota superior que uma inferior.

Observação 2.22. Em termos de construção de um conjunto de Cantor, não há nada de especial no valor $1 / 3$. De fato, dado $0<\nu<1$, se a cada passo tirarmos a $\nu$ - 
ésima parte central dos intervalos em questão, e tomarmos os pontos que restam no limite dessa construção, obtemos também um conjunto de Cantor, $C_{\nu}$, o qual tem dimensão de Hausdorff igual a $\frac{\log 2}{\log \left(\frac{1}{1-2 \nu}\right)} \in(0,1)$. Veja Folland [18].

Mais geral ainda, dada uma sequência $\left(a_{k}\right)_{k} \subset(0,1)$ tal que $a_{k} \rightarrow 0^{+}$, poderíamos considerar generalizações do conjunto de Cantor em que, na k-ésima etapa, removemos dos intervalos restantes suas porções médias de tamanho $a_{k}$. Este procedimento pode fazer com que o conjunto de Cantor resultante tenha dimensão de Hausdorff nula, como quando $\left(a_{k}\right)$ tem decaimento pelo menos geométrico. Consulte [5].

Dizemos que uma função $\mu^{*}: \mathcal{P}(X) \rightarrow[0, \infty]$ é uma medida exterior métrica em $X$ se satisfaz as seguintes propriedades:

(i) $\mu^{*}(\varnothing)=0$;

(ii) $\mu^{*}(A) \leqslant \mu^{*}(B)$, sempre que $A \subset B$;

(iii) dada uma coleção $\left\{A_{j}\right\}_{j=1}^{\infty}$ em $\mathcal{P}(X)$, tem-se

$$
\mu^{*}\left(\bigcup_{j=1}^{\infty} A_{j}\right) \leqslant \sum_{j=1}^{\infty} \mu^{*}\left(A_{j}\right)
$$

(iv) $\mu^{*}(A \cup B)=\mu^{*}(A)+\mu^{*}(B)$, sempre que $\mathrm{d}(A, B)>0$.

Dizemos ainda que um subconjunto $E \subset X$ é $\mu^{*}$-mensurável se para cada $A \subset X$ vale

$$
\mu^{*}(A)=\mu^{*}(A \cap E)+\mu^{*}\left(A \cap E^{c}\right) .
$$

Acontece que uma medida exterior métrica em $X$ é "capaz" mensurar qualquer conjunto boleriano de $X$.

Proposição 2.23. Se $\mu^{*}$ é uma medida exterior métrica em $X$, então todo subconjunto de Borel de X é $\mu^{*}$-mensurável.

Demonstração: Uma vez que os conjuntos fechados de $X$ geram a $\sigma$-álgebra de Borel de $X$, é suficiente mostrar que todo fechado de $X$ é $\mu^{*}$-mensurável. 
Seja $F \subset X$ fechado.

Dado $A \subset X$, com $\mu^{*}(A)<\infty$, queremos mostrar que

$$
\mu^{*}(A) \geqslant \mu^{*}(A \cap F)+\mu^{*}\left(A \cap F^{C}\right) .
$$

Para cada $n \in \mathbb{N}$, seja $B_{n}=A \cap\left(\mathcal{O}_{1 / n}(F)\right)^{C}$, de modo que $\left(B_{n}\right)_{n}$ é uma sequência crescente de conjuntos e, como $F$ é fechado, $A \cap F^{C}=\bigcup_{n \in \mathbb{N}} B_{n}$.

Como $\mu^{*}$ é uma medida exterior métrica e $\mathrm{d}\left(B_{n}, F\right) \geqslant 1 / n$, temos que

$$
\begin{aligned}
\mu^{*}(A) & =\mu^{*}\left((A \cap F) \cup\left(A \cap F^{C}\right)\right) \\
& \geqslant \mu^{*}\left((A \cap F) \cup B_{n}\right) \\
& =\mu^{*}(A \cap F)+\mu^{*}\left(B_{n}\right) .
\end{aligned}
$$

Basta então mostrarmos que vale a

Afirmação: $\mu^{*}\left(B_{n}\right) \rightarrow \mu^{*}\left(A \cap F^{C}\right)$.

Note que, pondo $C_{n}=B_{n+1} \cap B_{n}^{C}$, tem-se que

$$
\mathrm{d}\left(C_{n}, B_{n-1}\right) \geqslant \frac{1}{n-1}-\frac{1}{n}
$$

pois caso contrário, existiriam $x \in C_{n}$ e $y \in B_{n-1}$ tais que $\mathrm{d}(x, y)<\frac{1}{n-1}-\frac{1}{n}$. Como $\mathrm{d}\left(B_{n}, F\right) \geqslant 1 / n$, segue que $\mathrm{d}(y, F) \leqslant \mathrm{d}(y, x)+\mathrm{d}(x, F)<\left(\frac{1}{n-1}-\frac{1}{n}\right)+\frac{1}{n}$, de modo que $y \notin B_{n-1}$, uma contradição!

Consequentemente,

$$
\mu^{*}\left(B_{2 k+1}\right) \geqslant \sum_{j=1}^{k} \mu^{*}\left(C_{2 j}\right) \text { e } \mu^{*}\left(B_{2 k}\right) \geqslant \sum_{j=1}^{k} \mu^{*}\left(C_{2 j-1}\right)
$$

isto porque

$$
\begin{aligned}
\mu^{*}\left(B_{2 k+1}\right) & =\mu^{*}\left(\left(B_{2 k+1} \cap B_{2 k}^{C}\right) \cup\left(B_{2 k+1} \cap B_{2 k}\right)\right) \\
& \geqslant \mu^{*}\left(C_{2 k} \cup B_{2 k-1}\right) \\
& =\mu^{*}\left(C_{2 k}\right)+\mu^{*}\left(B_{2 k-1}\right)
\end{aligned}
$$


e procendendo por indução, obtemos a primeira desigualdade; e analogamente, a segunda.

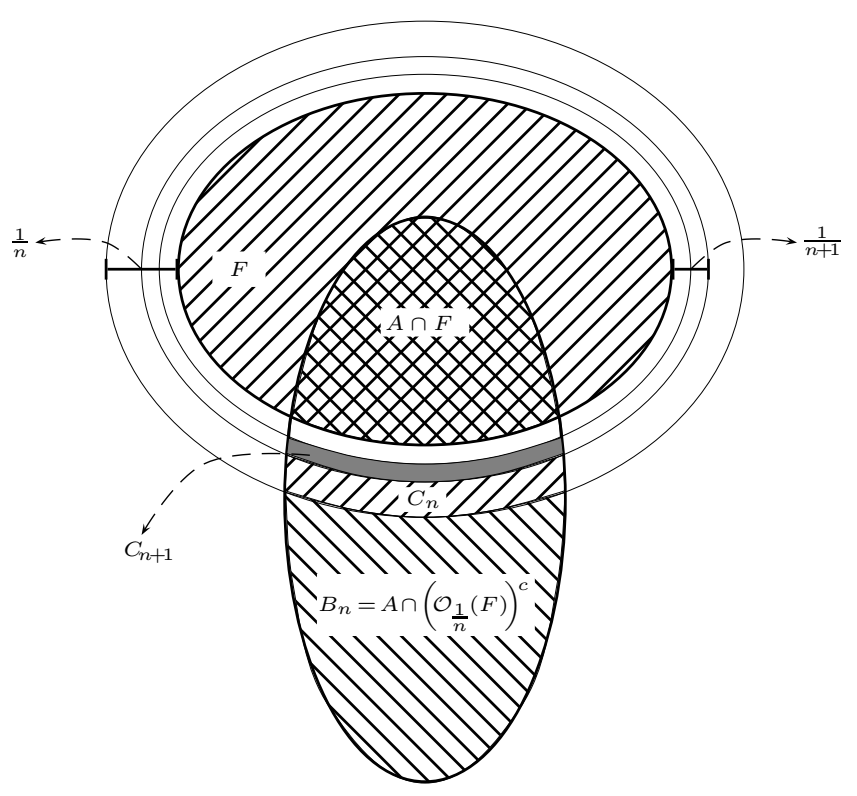

Como $\mu^{*}\left(B_{n}\right) \leqslant \mu^{*}(A)<\infty$, segue que as séries $\sum_{j=1}^{\infty} \mu^{*}\left(C_{2 j}\right)$ e $\sum_{j=1}^{\infty} \mu^{*}\left(C_{2 j-1}\right)$ são convergentes, e por subaditividade, temos

$$
\begin{aligned}
\mu^{*}\left(A \cap F^{C}\right) & =\mu^{*}\left(\left(B_{1} \cup \ldots \cup B_{n}\right) \cup\left(B_{n+1} \backslash B_{n}\right) \cup\left(B_{n+2} \backslash B_{n+1}\right) \cup \ldots\right) \\
& \leqslant \mu^{*}\left(B_{n}\right)+\sum_{j=n}^{\infty} \mu^{*}\left(C_{j}\right)
\end{aligned}
$$

de modo que

$$
\mu^{*}\left(A \cap F^{C}\right) \leqslant \liminf _{n \rightarrow \infty} \mu^{*}\left(B_{n}\right) \leqslant \limsup _{n \rightarrow \infty} \mu^{*}\left(B_{n}\right) \leqslant \mu^{*}\left(A \cap F^{C}\right)
$$

e isto encerra a demonstração.

Teorema 2.24. Fixados $\alpha \geqslant 0$ e $\delta>0$, valem:

i) $\mu_{\delta}^{(\alpha)}: \mathcal{P}(X) \mapsto[0, \infty]$ é uma medida exterior;

ii) $\mu^{(\alpha)}: \mathcal{P}(X) \mapsto[0, \infty]$ é uma medida exterior métrica invariante por isometrias de $X$; 
Consequentemente, dada uma coleção enumerável $\left\{A_{j}\right\}_{j}$ de subconjuntos de $X$, tem-se

$$
\operatorname{dim}_{\mathrm{H}}\left(\bigcup_{j=1}^{\infty} A_{j}\right)=\sup _{j \in \mathbb{N}} \operatorname{dim}_{\mathrm{H}}\left(A_{j}\right)
$$

Demonstração: Dados $\varepsilon \in(0, \delta / 2)$ e $a \in X$, a bola $B_{X}(a, \varepsilon)$ é uma cobertura enumerável de $\varnothing$, daí $\mu_{\delta}^{(\alpha)}(\varnothing) \leqslant\left(\operatorname{diam}\left(B_{X}(a, \varepsilon)\right)\right)^{\alpha}=(2 \varepsilon)^{\alpha} \stackrel{\varepsilon \rightarrow 0^{+}}{\longrightarrow} 0$, logo $\mu_{\delta}^{(\alpha)}(\varnothing)=0$. E também $\mu^{(\alpha)}(\varnothing)=0$.

Claro que $\mu_{\delta}^{(\alpha)}(A) \leqslant \mu_{\delta}^{(\alpha)}(B)$, sempre que $A \subset B$, uma vez que toda $\delta$-cobertura de $B$ é uma $\delta$-cobertura de $A$. Disso segue também que $\mu^{(\alpha)}(A) \leqslant \mu^{(\alpha)}(B)$, sempre que $A \subset B$.

Tome $\varepsilon>0$ e $\left\{A_{j}\right\}_{j}$ uma família enumerável de subconjuntos de $X$, e já podemos assumir que $\sum_{j=1}^{\infty} \mu_{\delta}^{(\alpha)}\left(A_{j}\right)<\infty$. Para cada $j$, seja $\left\{B_{i}^{j}\right\}_{i}$ uma $\delta$-cobertura de $A_{j}$ tal que

$$
\sum_{i=1}^{\infty}\left(\operatorname{diam}\left(B_{i}^{j}\right)\right)^{\alpha} \leqslant \mu_{\delta}^{(\alpha)}\left(A_{j}\right)+\frac{\varepsilon}{2^{j}}
$$

Com isso, $\left\{B_{i}^{j}\right\}_{i, j}$ forma uma $\delta$-cobertura para $\bigcup_{j=1}^{\infty} A_{j}$ e então

$$
\mu_{\delta}^{(\alpha)}\left(\bigcup_{j=1}^{\infty} A_{j}\right) \leqslant \sum_{i, j=1}^{\infty}\left(\operatorname{diam}\left(B_{i}^{j}\right)\right)^{\alpha} \leqslant \sum_{j=1}^{\infty} \mu_{\delta}^{(\alpha)}\left(A_{j}\right)+\varepsilon
$$

o que faz de $\mu_{\delta}^{(\alpha)}$ uma medida exterior.

E também, uma vez que $\mu_{\delta}^{(\alpha)}\left(A_{j}\right) \leqslant \mu^{(\alpha)}\left(A_{j}\right)$, para todo $\delta>0$ e todo $j$ natural, obtém-se

$$
\sum_{j=1}^{\infty} \mu^{(\alpha)}\left(A_{j}\right) \geqslant \sum_{j=1}^{\infty} \mu_{\delta}^{(\alpha)}\left(A_{j}\right) \geqslant \mu_{\delta}^{(\alpha)}\left(\bigcup_{j=1}^{\infty} A_{j}\right),
$$

para todo $\delta>0$; consequentemente,

$$
\mu^{(\alpha)}\left(\bigcup_{j=1}^{\infty} A_{j}\right)=\sup _{\delta>0} \mu_{\delta}^{(\alpha)}\left(\bigcup_{j=1}^{\infty} A_{j}\right) \leqslant \sum_{j=1}^{\infty} \mu^{(\alpha)}\left(A_{j}\right) .
$$

É claro que $\mu^{(\alpha)}$ é invariante por isometrias. Assim, para o item ii), resta mostrar que, 
dados $A, B \subset X$ e $\delta>0$ tais que $\mathrm{d}(A, B)>2 \delta$, vale

$$
\mu^{(\alpha)}(A \cup B) \geqslant \mu^{(\alpha)}(A)+\mu^{(\alpha)}(B)
$$

uma vez que a desigualdade oposta segue do fato de $\mu^{(\alpha)}$ ser uma medida exterior.

Na definição de $\mu_{\delta}^{(\alpha)}(A \cup B)$, podemos nos restringir às coberturas que de fato interceptam $A \cup B$, de modo que $\left(C_{j}\right)_{j}$ é uma $\delta$-cobertura de $A \cup B$ se, e somente se, $\left(A \cap C_{j}\right)_{j}$ e $\left(B \cap C_{j}\right)_{j}$ são $\delta$-coberturas de $A$ e $B$, respectivamente; e neste caso, estas $\delta$-coberturas são disjuntas, pela escolha de $\delta$.

Assim, pondo $A_{j}=A \cap C_{j}$ e $B_{j}=B \cap C_{j}$,

$$
\mu_{\delta}^{(\alpha)}(A \cup B)=\inf \left\{\sum_{j \in \mathbb{N}}\left(\operatorname{diam}\left(A_{j}\right)\right)^{\alpha}+\sum_{j \in \mathbb{N}}\left(\operatorname{diam}\left(B_{j}\right)\right)^{\alpha}\right\}
$$

obtemos, para cada $\eta>0, \delta$-coberturas $\left\{A_{j}\right\}_{j}$ e $\left\{B_{j}\right\}_{j}$ de $A$ e $B$, respectivamente, tais que

$$
\mu_{\delta}^{(\alpha)}(A \cup B)+\eta \geqslant \sum_{j \in \mathbb{N}}\left(\operatorname{diam}\left(A_{j}\right)\right)^{\alpha}+\sum_{j \in \mathbb{N}}\left(\operatorname{diam}\left(B_{j}\right)\right)^{\alpha} \geqslant \mu_{\delta}^{(\alpha)}(A)+\mu_{\delta}^{(\alpha)}(B) .
$$

Agora, basta passar o limite $\operatorname{com} \delta \rightarrow 0^{+}$para concluir, dada a arbitrariedade de $\eta>0$, que $\mu^{(\alpha)}(A \cup B) \geqslant \mu^{(\alpha)}(A)+\mu^{(\alpha)}(B)$.

Finalmente, seja $\left\{A_{j}\right\}_{j} \subset X$ uma família enumerável de subconjuntos de $X$. Por monotonicidade segue que

$$
\sup _{j \in \mathbb{N}} \operatorname{dim}_{\mathrm{H}}\left(A_{j}\right) \leqslant \operatorname{dim}_{\mathrm{H}}\left(\bigcup_{j=1}^{\infty} A_{j}\right)
$$

e usando que $\operatorname{dim}_{\mathrm{H}}\left(A_{j}\right)$ é o ínfimo $\operatorname{dos} \alpha>0$ tais que $\mu^{(\alpha)}\left(A_{j}\right)=0$, da Proposição 2.21, segue que, dado $\alpha>\sup \operatorname{dim}_{\mathrm{H}}\left(A_{j}\right)$, temos $\mu^{(\alpha)}\left(\bigcup A_{j}\right) \leqslant \sum \mu^{(\alpha)}\left(A_{j}\right)=0$, consequentemente $\operatorname{dim}_{\mathrm{H}}\left(\bigcup A_{j}\right) \leqslant \alpha$.

Uma vez que $\alpha>\sup _{j \in \mathbb{N}} \operatorname{dim}_{\mathrm{H}}\left(A_{j}\right)$ é arbitrário, segue a outra desigualdade

$$
\sup _{j \in \mathbb{N}} \operatorname{dim}_{\mathrm{H}}\left(A_{j}\right) \geqslant \operatorname{dim}_{\mathrm{H}}\left(\bigcup_{j=1}^{\infty} A_{j}\right)
$$


Este resultado tem importantes consequências, a serem verificadas cada uma a seu tempo, tais como:

1) se $E \subset X$ é enumerável, então $\operatorname{dim}_{\mathrm{H}}(E)=0$;

2) se $I$ é um intervalo de extremos $a<b$, então $\mu^{(1)}(I)=b-a$ e consequentemente $\operatorname{dim}_{\mathrm{H}}(I)=1$;

3) se $A \subset \mathbb{R}^{m}$, então $0 \leqslant \operatorname{dim}_{\mathrm{H}}(A) \leqslant m$;

4) todo aberto de $\mathbb{R}^{m}$ tem dimensão de Hausdorff $m$;

5) $\operatorname{dim}_{\mathrm{H}}\left(\mathbb{R}^{m}\right)=m$;

6) toda $m$-variedade $M \subset \mathbb{R}^{m+n}$ tem dimensão de Hausdorff $m$;

7) a $\alpha$-medida de Hausdorff restrita à $\sigma$-álgebra de Borel de $X$ fornece uma medida, pelo Teorema de Carathéodory (Teorema 1.201).

Sabendo que $\mu^{(\alpha)}$ é uma medida exterior e que pontos têm $\alpha$-medida de Hausdorff nula, concluímos que $\operatorname{dim}_{\mathrm{H}}(E)=0$, sempre que $E$ é enumerável; de onde segue o item 1 ) da lista acima.

Proposição 2.25. Se $E \subset X$ é enumerável, então $\operatorname{dim}_{\mathrm{H}}(E)=0$.

Teorema 2.26. Se I é um intervalo de extremos $a<b$, então $\mu^{(1)}(I)=b-a$ e consequentemente

$$
\operatorname{dim}_{\mathrm{H}}(I)=1
$$

Em particular, $\operatorname{dim}_{H}(\mathbb{R})=1$.

\section{Demonstração:}

Seja $I$ um intervalo de extremos $a<b$. Inicialmente, tome $I=[a, b]$ e mostremos que $\mu^{(1)}([a, b]) \leqslant b-a$.

Dado $\delta \in(0, b-a)$, tome $N \in \mathbb{N}$ tal que $h=\frac{b-a}{N}<\frac{\delta}{2}$ e considere a coleção $\left\{I_{1}, I_{2}, \ldots, I_{N}\right\}$ em que $I_{1}=\left[a, a+h+\frac{\delta}{N}\right)$

$$
I_{n}=\left(a+(n-1) h-\frac{\delta}{2 N}, a+n h+\frac{\delta}{2 N}\right)
$$


para $n=2, \ldots, N-1$, e $I_{N}=\left(a+(N-1) h-\frac{\delta}{N}, b\right]$. E uma vez que, para cada $n=1,2, \ldots, N, \operatorname{diam}\left(I_{n}\right)=h+\frac{\delta}{N}<h+\frac{b-a}{N}<\delta$, segue que $\left\{I_{1}, I_{2}, \ldots, I_{N}\right\}$ é uma $\delta$-cobertura de $[a, b]$, de modo que

$$
\sum_{n=1}^{N} \operatorname{diam}\left(I_{n}\right)=N\left(h+\frac{\delta}{N}\right)=N h+\delta=b-a+\delta
$$

daí $\mu_{\delta}^{(1)}([a, b]) \leqslant b-a+\delta, \forall \delta \in(0, b-a), \log 0 \mu^{(1)}([a, b]) \leqslant b-a$.

Por outro lado, pelo Lema 1.15, sejam $\left\{I_{j}\right\}_{j}$ uma cobertura aberta enumerável de $[a, b]$ e $\varepsilon$ um número de Lebesgue associado a ela, i.e., $\varepsilon>0$ tem a propriedade de

$$
x, y \in[a, b] \operatorname{com}|x-y|<\varepsilon \Rightarrow x, y \in I_{j}, \text { para algum } j,
$$

Tome $N \in \mathbb{N}$ tal que $h=\frac{b-a}{N}<\varepsilon$, daí para cada $n \in\{1,2, \ldots, N\}$, seja $j_{n} \in \mathbb{N}$ tal que $a+(n-1) h, a+n h \in I_{j_{n}} ;$ em particular, $h<\operatorname{diam}\left(I_{j_{n}}\right)$. Assim,

$$
\begin{aligned}
b-a=N h & =\sum_{n=1}^{N}((a+n h)-(a+(n-1) h)) \\
& <\sum_{n=1}^{N} \operatorname{diam}\left(I_{j_{n}}\right) \\
& \leqslant \sum_{j \in \mathbb{N}} \operatorname{diam}\left(I_{j}\right),
\end{aligned}
$$

qualquer que seja $\left\{I_{j}\right\}_{j}$ cobertura aberta enumerável de $[a, b]$.

Logo, $b-a \leqslant \mu^{(1)}([a, b])$, e consequentemente

$$
\mu^{(1)}([a, b])=b-a .
$$

Para outros intervalos, basta observar que

$$
\begin{aligned}
& (a, b]=\bigcup_{k \in \mathbb{N}}[a+1 / k, b], \\
& {[a, b)=\bigcup_{k \in \mathbb{N}}[a, b-1 / k]}
\end{aligned}
$$


$\mathrm{e}$

$$
(a, b)=\bigcup_{k \in \mathbb{N}}[a+1 / k, b-1 / k]
$$

e então aplicar o Teorema 2.24.

Além disso,

$$
\operatorname{dim}_{\mathrm{H}}(\mathbb{R})=\operatorname{dim}_{\mathrm{H}}\left(\bigcup_{k \in \mathbb{Z}}[k, k+1]\right)=\sup _{k \in \mathbb{Z}} \operatorname{dim}_{\mathrm{H}}([k, k+1])=1 .
$$

O próximo lema fornece condições para que $\mu^{(\alpha)}\left(\bigcup_{j \in \mathbb{N}} A_{j}\right)=\lim _{j \rightarrow \infty} \mu^{(\alpha)}\left(A_{j}\right)$.

Lema 2.27. Sejam $(X, \mathrm{~d})$ um espaço métrico e $\left\{A_{j}\right\}_{j=1}^{\infty}$ uma sequência crescente de subconjuntos de $X$.

Se $A=\bigcup_{j \in \mathbb{N}} A_{j}, \mu^{\alpha}(A)<\infty$ e $\mathrm{d}\left(A_{j}, A \backslash A_{j+1}\right)>0$ para cada $j \in \mathbb{N}$, então

$$
\mu^{(\alpha)}(A)=\lim _{j \rightarrow \infty} \mu^{(\alpha)}\left(A_{j}\right)
$$

Demonstração: Da monotonicidade de $\left\{A_{j}\right\}_{j}$, segue que $\lim \mu^{(\alpha)}\left(A_{j}\right)$ existe e

$$
\lim _{j \rightarrow \infty} \mu^{(\alpha)}\left(A_{j}\right) \leqslant \mu^{(\alpha)}(A)
$$

Sejam $B_{1}=A_{1}$ e $B_{j}=A_{j} \backslash A_{j-1}$, para $j=2,3, \ldots$

Note que $\left\{B_{2 j}\right\}_{j}$ é dois a dois positivamente separada, pois $B_{2 j} \subset A_{2 j}$ e $B_{2 j+2 k} \subset$ $A \backslash A_{2 j+1}$ implicam que $\mathrm{d}\left(B_{2 j}, B_{2 j+2 k}\right) \geqslant \mathrm{d}\left(A_{2 j}, A \backslash A_{2 j+1}\right)>0$. O mesmo vale para $\left\{B_{2 j-1}\right\}_{j}$.

Com isso,

$$
\mu^{(\alpha)}(A) \geqslant \mu^{(\alpha)}\left(\bigcup_{j=1}^{n} B_{2 j-1}\right)=\sum_{j=1}^{n} \mu^{(\alpha)}\left(B_{2 j-1}\right)
$$

e

$$
\mu^{(\alpha)}(A) \geqslant \mu^{(\alpha)}\left(\bigcup_{j=1}^{n} B_{2 j}\right)=\sum_{j=1}^{n} \mu^{(\alpha)}\left(B_{2 j}\right)
$$


daí para cada $n \in \mathbb{N}$,

$$
2 \mu^{(\alpha)}(A) \geqslant \sum_{j=1}^{n} \mu^{(\alpha)}\left(B_{j}\right)
$$

o que implica que a série $\sum_{j=1}^{\infty} \mu^{(\alpha)}\left(B_{j}\right)$ é convergente.

Finalmente, tomando o limite com $n \rightarrow \infty$ em

$$
\mu^{(\alpha)}(A)=\mu^{(\alpha)}\left(\bigcup_{j \in \mathbb{N}} A_{j}\right) \leqslant \mu^{(\alpha)}\left(A_{n}\right)+\sum_{j=n+1}^{\infty} \mu^{(\alpha)}\left(B_{j}\right),
$$

segue o resultado.

Este próximo resultado será lembrado até o fim deste texto, pois fornece condições sobre uma função $f: X \rightarrow X$ para que possamos relacionar $\operatorname{dim}_{\mathrm{H}}(f(A))$ e $\operatorname{dim}_{\mathrm{H}}(A)$, o que é crucial na demonstração do Teorema de Mañé (Teorema 3.6).

Dados espaços métricos $(X, \mathrm{~d})$ e $\left(Y, \mathrm{~d}^{\prime}\right)$ e uma função $g: X \rightarrow Y$, dizemos que $g$ é uma função $C$-Lipschitz se é uma função Lipschitz com constante de Lipschitz igual a $C$, i.e.,

$$
\mathrm{d}^{\prime}(g(x), g(y)) \leqslant C \mathrm{~d}(x, y)
$$

para todo $x, y \in X$.

Quando $g$ é uma bijeção e tanto $g$ quanto $g^{-1}$ são funções Lipschitz, diremos que $g$ é uma função bi-Lipschitz. Enquanto homeomorfismos dizem quando dois conjuntos são "iguais" do ponto de vista topológico, as aplicações bi-Lipschitz dizem quando dois conjuntos são "iguais" do ponto de vista da geometria fractal. Veremos que a dimensão de Hausdorff e a dimensão fractal são invariantes por aplicações bi-Lipschitz.

Teorema 2.28. Sejam $(X, \mathrm{~d})$ um espaço métrico e $A \subset X$. Se $f: X \rightarrow X$ é uma função C-Lipschitz, então

a) $\mu^{(\alpha)}(f(A)) \leqslant C^{\alpha} \mu^{(\alpha)}(A)$

b) $\operatorname{dim}_{\mathrm{H}}(f(A)) \leqslant \operatorname{dim}_{\mathrm{H}}(A)$

c) $\operatorname{dim}_{\mathrm{H}}\left(\operatorname{Graf}\left(\left.f\right|_{A}\right)\right)=\operatorname{dim}_{\mathrm{H}}(A)$

Demonstração: O item a) decorre da seguinte afirmação, substituindo $g$ pela aplicação identidade e pondo $Y=X$ : 
Afirmação: suponha $f, g: Y \subset X \rightarrow X$ tais que $\mathrm{d}(f(x), f(y)) \leqslant C \mathrm{~d}(g(x), g(y)), \forall x, y \in$ $Y$, então para todo $A \subset Y$ vale

$$
\mu^{(\alpha)}(f(A)) \leqslant C^{\alpha} \mu^{(\alpha)}(g(A))
$$

Suponha $\mu^{(\alpha)}(g(A))<\infty$.

Dado $\varepsilon>0$, para cada $\delta>0$, existe uma $C^{-1} \delta$-cobertura de $g(A)$ por conjuntos $B_{j} \subset X$ tal que

$$
\sum_{j=1}^{\infty}\left(\operatorname{diam}\left(B_{j}\right)\right)^{\alpha} \leqslant \mu^{(\alpha)}(g(A))+\varepsilon .
$$

Note que $\tilde{B}_{j}=f\left(g^{-1}\left(B_{j}\right)\right)$ cobrem $f(A)$ : como $g(A) \subset \bigcup B_{j}$, segue que $A \subset$ $g^{-1}(g(A)) \subset \bigcup g^{-1}\left(B_{j}\right)$, daí $f(A) \subset \bigcup f\left(g^{-1}\left(B_{j}\right)\right)$.

E mais, $\operatorname{diam}\left(\tilde{B}_{j}\right) \leqslant C \operatorname{diam}\left(B_{j}\right)<\delta$, consequentemente

$$
\begin{aligned}
\mu_{\delta}^{(\alpha)}(f(A)) & \leqslant \sum_{j=1}^{\infty}\left(\operatorname{diam}\left(\tilde{B}_{j}\right)\right)^{\alpha} \\
& \leqslant C^{\alpha} \sum_{j=1}^{\infty}\left(\operatorname{diam}\left(B_{j}\right)\right)^{\alpha} \\
& \leqslant C^{\alpha} \mu^{(\alpha)}(g(A))+\varepsilon C^{\alpha}
\end{aligned}
$$

e agora fazendo $\delta \rightarrow 0^{+}$e $\varepsilon \rightarrow 0^{+}$, obtemos

$$
\mu^{(\alpha)}(f(A)) \leqslant C^{\alpha} \mu^{(\alpha)}(g(A))
$$

Para o item b), como $\mu^{(\alpha)}(A)=0$ implica $\mu^{(\alpha)}(f(A))=0$, do item anterior, obtemos

$$
\left\{\alpha>0: \mu^{(\alpha)}(A)=0\right\} \subset\left\{\alpha>0: \mu^{(\alpha)}(f(A))=0\right\}
$$

e então $\operatorname{dim}_{\mathrm{H}}(f(A)) \leqslant \operatorname{dim}_{\mathrm{H}}(A)$.

Quanto ao item c), como as aplicações

$$
A \ni x \longmapsto(x, f(x)) \in \operatorname{Graf}\left(\left.f\right|_{A}\right)
$$


$\mathrm{e}$

$$
\operatorname{Graf}\left(\left.f\right|_{A}\right) \ni(x, f(x)) \longmapsto x \in A
$$

são Lipschitz, segue que

$$
\operatorname{dim}_{\mathrm{H}}\left(\operatorname{Graf}\left(\left.f\right|_{A}\right)\right) \leqslant \operatorname{dim}_{\mathrm{H}}(A) \leqslant \operatorname{dim}_{\mathrm{H}}\left(\operatorname{Graf}\left(\left.f\right|_{A}\right)\right)
$$

\section{Proposição 2.29. (Desigualdade Isodiamétrica, Diestel [10], Theorem 1.8)}

Se $E \subset \mathbb{R}^{m}$ é um boreliano, então

$$
\mathcal{L}^{m}(E) \leqslant\left(\frac{\sqrt{\pi^{m}}}{2^{m} \Gamma\left(\frac{m}{2}+1\right)}\right) \operatorname{diam}(E)^{m}
$$

Novamente, é a limitação inferior que mais demanda esforços: o resultado acima é necessário para mostrar que a $m$-medida de Hausdorff de um cubo é estritamente positiva.

Teorema 2.30. Se $Q \subset \mathbb{R}^{m}$ é um cubo, então $0<\mu^{(m)}(Q)<\infty$.

Em particular,

$$
\operatorname{dim}_{\mathrm{H}}(Q)=m
$$

\section{Demonstração:}

Sem perda de generalidade, assuma que $Q$ tem lado unitário.

Dividindo $Q$ em $k^{m}$ subcubos de lado $1 / k$ da maneira canônica, de modo que cada um deles tem diâmetro $\delta_{k}=\sqrt{m} / k$, e então

$$
\mu_{\delta_{k}}^{(m)}(Q) \leqslant k^{m}(\sqrt{m} / k)^{m}=\sqrt{m^{m}}
$$

de modo que $\mu^{(m)}(Q)<\infty$.

Dada uma cobertura aberta enumerável $\left\{A_{j}\right\}_{j}$ de $Q$, a Proposição 2.29 fornece uma constante $b_{m}>0$ tal que $\mathcal{L}^{m}\left(A_{j}\right) \leqslant b_{m} \operatorname{diam}\left(A_{j}\right)^{m}$, daí

$$
1=\mathcal{L}^{m}(Q) \leqslant \sum_{j=1}^{\infty} \mathcal{L}^{m}\left(A_{j}\right) \leqslant \sum_{j=1}^{\infty} b_{m} \operatorname{diam}\left(A_{j}\right)^{m}
$$


$\log 0$

$$
\sum_{j=1}^{\infty} \operatorname{diam}\left(A_{j}\right)^{m} \geqslant 1 / b_{m} .
$$

Sendo assim, $\mu_{\delta}^{(m)}(Q) \geqslant 1 / b_{m}$, para todo $\delta>0$; consequentemente $\mu^{(m)}(Q)>0$.

Corolário 2.31. Para todo $m$ natural, $\mathbb{R}^{m}$ tem dimensão de Hausdorff $m$.

Consequentemente,

$$
0 \leqslant \operatorname{dim}_{\mathrm{H}}(A) \leqslant m
$$

para todo $A \subset \mathbb{R}^{m}$.

\section{Demonstração:}

Uma vez que podemos escrever $\mathbb{R}^{m}$ como uma reunião enumerável de cubos $Q$ de lado unitário, segue do Teorema 2.24 e do Teorema 2.30 que $\operatorname{dim}_{H}\left(\mathbb{R}^{m}\right)=m$.

Mais geralmente, o Corolário 2.31 vale para qualquer espaço de Banach $X$ com dimensão $m$, pois este é isometricamente isomorfo a $\mathbb{R}^{m}$.

Corolário 2.32. Se $A \subset \mathbb{R}^{m}$ tem interior não vazio, então $\operatorname{dim}_{\mathrm{H}}(A)=m$.

\section{Demonstração:}

Dado $x_{0} \in A$, existe um cubo $Q$ tal que $x_{0} \in Q \subset A$. Pelo Teorema 2.30 e pelo Corolário 2.31,

$$
m=\operatorname{dim}_{\mathrm{H}}(Q) \leqslant \operatorname{dim}_{\mathrm{H}}(A) \leqslant \operatorname{dim}_{\mathrm{H}}\left(\mathbb{R}^{m}\right)=m .
$$

A íntima relação entre a medida de Lebesgue em $\mathbb{R}^{m}, \mathcal{L}^{m}$, e a $m$-medida de Hausdorff, a qual foi sugerida no Teorema 2.30 advém do fato de que medidas de Haar em $\mathbb{R}^{m}$ são únicas, a menos de um ajuste por uma constante conveniente. Em outras palavras, existe $c_{m}>0$ tal que

$$
\mathcal{L}^{m}(A)=c_{m} \mu^{(m)}(A), \forall A \subset \mathbb{R}^{m}
$$

Quando $m=1$, as definições coincidem, o que já havia sido visto quando provamos que a 1-medida de Hausdorff de um intervalo real é o seu comprimento. A constante $c_{m}$ 
é de fato o volume de uma bola de diâmetro unitário: $c_{m}=\frac{\sqrt{\pi^{m}}}{2^{m} \Gamma\left(\frac{m}{2}+1\right)}$. Há autores inclusive que definem a $\alpha$-medida de Hausdorff como sendo $c_{\alpha} \mu^{(\alpha)}$, em que

$$
c_{\alpha}=\frac{\sqrt{\pi^{\alpha}}}{2^{\alpha} \Gamma\left(\frac{\alpha}{2}+1\right)} .
$$

Consulte o Apêndice D para um tratamento mais detalhado.

Foi importante constatar que $\operatorname{dim}_{\mathrm{H}}\left(\mathbb{R}^{m}\right)=m$, pois com isso a dimensão de Hausdorff concorda com a dimensão topológica e com a dimensão de espaço vetorial com as quais somos mais familiares. E isto nos dá o direito de chamar a dimensão de Hausdorff de "dimensão"; e não apenas de uma função estranha de $\mathcal{P}(X)$ em $[0, \infty]$.

Agora fica fácil verificar que toda $m$-variedade $M \subset \mathbb{R}^{m+n}$ tem dimensão de Hausdorff $m$ : sabendo que $M$ é, em particular, subconjunto de um espaço métrico separável, $M$ é reunião enumerável de abertos relativos $U \subset M$ homeomorfos a abertos de $\mathbb{R}^{m}$, ou seja, $M$ é reunião enumerável de conjuntos com dimensão de Hausdorff $m$.

Em particular, a esfera $S^{m} \subset \mathbb{R}^{m+1}$ tem dimensão de Hausdorff $m$.

\subsection{A Dimensão Fractal}

Um ponto em comum entre as dimensões fractais é a análise local dos conjuntos, em busca de irregularidades ou de autossimilaridade, uma propriedade típica de conjuntos fractais. A dimensão de Hausdorff conseguia enxergar os conjuntos a esse nível "microscópico", pois se fazia valer de $\delta$-coberturas com $\delta$ arbitrariamente pequeno. Enxergar a tal nível é a chave para detectar propriedades fractais, algo que a dimensão topológica é incapaz de fazer.

Seja $X$ um espaço métrico compacto. Fixemos $N(r, X)$ o número mínimo de bolas de $X$ de raio $r>0$ centradas em pontos de $X$ necessárias para cobrir $X$.

Podemos nos perguntar quão rápido $N(r, X)$ cresce em resposta a $r \rightarrow 0^{+}$; o que em um certo sentido é investigar como $X$ adquire alguma regularidade ou não, a medida que se enxerga o conjunto numa escala maior ( $r$ menor). Dadas duas grandezas $a r^{\alpha}$ e $b r^{\beta}$, note que

$$
\lim _{r \rightarrow 0^{+}} \frac{\log a r^{\alpha}}{\log b r^{\beta}}=\frac{\alpha}{\beta}
$$

de modo que, na escala log-log, conseguimos mensurar de que forma estas grandezas se 
relacionam quando $r \rightarrow 0^{+}$. A dimensão fractal fará este serviço: dirá, quando for possível dizer, como as grandezas $N(r, X)$ e $1 / r$ se compensam na escala log-log, quando $r \rightarrow 0^{+}$. Importante apontar que o limite acima pode não existir dependendo das irregularidades locais de $X$ (consulte o Exercício 3.8 em Falconer [16]). Isso justifica usarmos limite superior em vez de limite, o que dá origem à (upper) box-counting dimension, que chamaremos simplesmente de dimensão fractal.

A dimensão fractal de $X$, denotada por $\operatorname{dim}_{\mathrm{F}}(X)$, é dada por

$$
\operatorname{dim}_{\mathrm{F}}(X):=\limsup _{r \rightarrow 0^{+}} \frac{\log N(r, X)}{\log 1 / r}
$$

Para evitar problemas com " $\log 0 "$ e " $\log \infty "$, assumimos que $X$ é não vazio.

Note que, na verdade, a definição de dimensão fractal apenas exigiu que $X$ fosse totalmente limitado, de modo que podemos estendê-la a tais espaços métricos.

Uma vez que a dimensão fractal é determinada por coberturas formadas por finitos conjuntos de mesmo diâmetro e natureza, ela tende a ser mais fácil de ser calculada que a dimensão de Hausdorff, sem contar o fato de recorrer ao conceito de limsup, o que também é reconfortante, em muitas ocasiões.

Alternativamente, $\operatorname{dim}_{\mathrm{F}}(X)$ é o menor número para o qual, dado $\varepsilon>0$, existe $\delta>0$ tal que

$$
N(r, X) \leqslant\left(\frac{1}{r}\right)^{\operatorname{dim}_{\mathrm{F}}(X)+\varepsilon}, 0<r<\delta .
$$

De fato, como $\operatorname{dim}_{\mathrm{F}}(X)=\inf _{\delta>0} \sup _{0<r<\delta} \frac{\log N(r, X)}{\log 1 / r}$, dado $\varepsilon>0$, existe $\delta>0$ tal que

$$
\begin{aligned}
0 \leqslant \sup _{0<r<\delta} \frac{\log N(r, X)}{\log 1 / r} \leqslant \operatorname{dim}_{\mathrm{F}}(X)+\varepsilon & \Leftrightarrow \frac{\log N(r, X)}{\log 1 / r} \leqslant \operatorname{dim}_{\mathrm{F}}(X)+\varepsilon, \quad \forall r \in(0, \delta) \\
& \Leftrightarrow \log _{1 / r} N(r, X) \leqslant \operatorname{dim}_{\mathrm{F}}(X)+\varepsilon, \forall r \in(0, \delta) \\
& \Leftrightarrow N(r, X) \leqslant(1 / r)^{\operatorname{dim}_{\mathrm{F}}(X)+\varepsilon}, \forall r \in(0, \delta) .
\end{aligned}
$$

A dimensão fractal pode ainda ser calculada tomando o lim sup sobre uma sequência apropriada. Este modo particular de $r$ se aproximar de 0 é de grande ajuda, como veremos repetidas vezes.

Lema 2.33. Se $\left(a_{n}\right)_{n} \subset(0, \infty)$ é uma sequência decrescente convergente para 0 tal que 
$a_{n+1} \geqslant \alpha a_{n}$, para algum $0<\alpha<1$, então

$$
\operatorname{dim}_{\mathrm{F}}(X)=\limsup _{n \rightarrow \infty} \frac{\log N\left(a_{n}, X\right)}{-\log a_{n}}
$$

\section{Demonstração:}

Seja $c_{0}=\limsup _{n \rightarrow \infty} \frac{\log N\left(a_{n}, X\right)}{-\log a_{n}}$. Claro que $\operatorname{dim}_{\mathrm{F}}(X) \geqslant c_{0}$.

Dado $0<r<a_{1}$, tome $n$ tal que $a_{n+1} \leqslant r<a_{n}$, daí $N\left(a_{n+1}, X\right) \geqslant N(r, X)$ e $\log r<\log a_{n}$. Com isso,

$$
\frac{\log N(r, X)}{-\log r} \leqslant \frac{\log N\left(a_{n+1}, X\right)}{-\log a_{n}}=\frac{\log N\left(a_{n+1}, X\right)}{-\log a_{n+1}+\log \left(a_{n+1} / a_{n}\right)} \leqslant \frac{\log N\left(a_{n+1}, X\right)}{-\log a_{n+1}+\log \alpha}
$$

de onde obtemos

$$
\operatorname{dim}_{\mathrm{F}}(X)=\limsup _{r \rightarrow 0^{+}} \frac{\log N(r, X)}{-\log r} \leqslant \limsup _{n \rightarrow \infty} \frac{\log N\left(a_{n+1}, X\right)}{-\log a_{n+1}}=c_{0} .
$$

Em particular, vamos frequentemente considerar sequências $\left(a_{n}\right)_{n}$ dos tipos

$$
a_{n}=\nu^{n} r_{0}
$$

sendo $r_{0}>0$ e $\nu \in(0,1)$, e também

$$
a_{n}=\frac{r_{0}}{n}
$$

sendo $r_{0}>0$. Novamente, enfatizamos que esta é uma propriedade muito conveniente e que haverá muitas oportunidades de reconhecer isso; em especial para o cômputo da dimensão fractal de atratores exponenciais e ao longo de todo o Capítulo 4.

A esta altura, somos capazes de provar que $\operatorname{dim}_{\mathrm{H}}(X) \leqslant \operatorname{dim}_{\mathrm{F}}(X)$, para todo espaço métrico compacto $X$. No entanto, esta desigualdade é considerada apenas no Teorema 2.41 da Seção 2.4 para efeito de organização. Ainda assim, usaremo-na desde já para provar o seguinte lema.

Lema 2.34. Todo compacto $K \subset \mathbb{R}^{m}$ tem dimensão fractal no máximo $m$. 
Além disso, se $K$ contém alguma bola aberta, então

$$
\operatorname{dim}_{\mathrm{F}}(K)=m
$$

\section{Demonstração:}

Sejam $a<b$ tais que $K \subset[a, b]^{m}$. Note que podemos cobrir tal cubo $[a, b]^{m}$ por $(2 n)^{m}$ bolas de raio $\frac{b-a}{n}$, para cada $n$ natural, ou seja, $N\left(\frac{b-a}{n},[a, b]^{m}\right) \leqslant(2 n)^{m}$. Com isso,

$$
\operatorname{dim}_{\mathrm{F}}(K) \leqslant \operatorname{dim}_{\mathrm{F}}\left([a, b]^{m}\right)=\limsup _{n \rightarrow \infty} \frac{\log N\left(\frac{b-a}{n},[a, b]^{m}\right)}{\log \left(\frac{n}{b-a}\right)} \leqslant \limsup _{n \rightarrow \infty} \frac{m \log 2 n}{\log \left(\frac{n}{b-a}\right)}=m
$$

Agora, se $B_{\mathbb{R}^{m}}\left(x_{0}, r_{0}\right) \subset K$, para algum $x_{0} \in K$ e algum $r_{0}>0$, então

$$
m=\operatorname{dim}_{\mathrm{H}}\left(B_{\mathbb{R}^{m}}\left(x_{0}, r_{0}\right)\right) \leqslant \operatorname{dim}_{\mathrm{F}}(K) \leqslant m .
$$

Note que a dimensão fractal é monótona: se $K_{1} \subset K_{2}$, então $\operatorname{dim}_{\mathrm{F}}\left(K_{1}\right) \leqslant \operatorname{dim}_{\mathrm{F}}\left(K_{2}\right)$, simplesmente porque $N\left(r, K_{1}\right) \leqslant N\left(r, K_{2}\right)$, para cada $r>0$. Dito isto, é fácil verificar que é finitamente estável:

$$
\operatorname{dim}_{\mathrm{F}}\left(K_{1} \cup \cdots \cup K_{n}\right)=\max _{j=1, \ldots, n} \operatorname{dim}_{\mathrm{F}}\left(K_{j}\right)
$$

e, mais geralmente, vale

$$
\operatorname{dim}_{\mathrm{F}}\left(\bigcup_{j \in \mathbb{N}} K_{j}\right) \geqslant \sup _{j \in \mathbb{N}} \operatorname{dim}_{\mathrm{F}}\left(K_{j}\right)
$$

Lema 2.35. A dimensão fractal é invariante por aplicações bi-Lipschitz.

\section{Demonstração:}

Seja $f: X \rightarrow X$ uma bijeção $c$-Lipshitz com inversa $f^{-1} \tilde{c}$-Lipschitz.

Como $\mathrm{d}(f(x), f(y)) \leqslant c \mathrm{~d}(x, y)$, se $X$ pode ser coberto por $N(r, X)$ bolas de raio $r$, então as imagens dessas bolas por $f$ formam uma cobertura por $N(r, X)$ bolas de raio $c r$, 
o qual é maior ou igual a $N(c r, f(X))$, de onde obtemos que

$$
\operatorname{dim}_{\mathrm{F}}(f(X)) \leqslant \operatorname{dim}_{\mathrm{F}}(X)
$$

A outra desigualdade é obtida analogamente, usando a inversa $f^{-1}$.

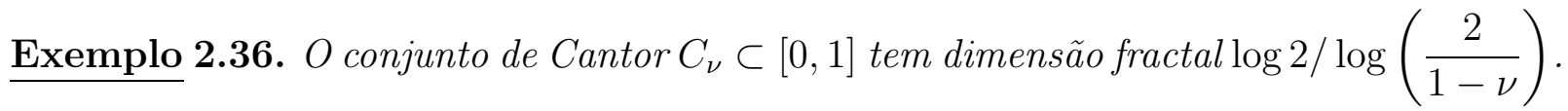

A construção do conjunto $C_{\nu}$, $\operatorname{com} \nu \in(0,1)$, se dá como segue: no primeiro passo, removemos de $[0,1]$ o intervalo concêntrico de comprimento $\nu$, restando então dois intervalos de tamanho $(1-\nu) / 2$; no segundo, destes removemos os dois respectivos intervalos concêntricos de tamanho $\nu(1-\nu) / 2$, restando agora quatro intervalos de tamanho $(1-\nu)^{2} / 2^{2}$. No $k$-ésimo passo, restam $2^{k}$ intervalos de comprimento $(1-\nu)^{k} / 2^{k}$. Definimos $C_{\nu}$ como sendo o conjunto dos pontos que permanecem após este procedimento ser feito indefinidamente.

Para o cômputo da sua dimensão fractal, os intervalos envolvidos na sua construção já são suficientes. Note que no $k$-ésimo passo, os extremos dos $2^{k}$ intervalos restantes não serão removidos nos próximos passos, de modo que tais intervalos formam a cobertura minimal por intervalos de comprimento $(1-\nu)^{k} / 2^{k}$, ou seja, $N\left((1-\nu)^{k} / 2^{k+1}, C_{\nu}\right)=2^{k}$. Assim,

$$
\operatorname{dim}_{\mathrm{F}}\left(C_{\nu}\right)=\limsup _{k \rightarrow \infty} \frac{\log N\left((1-\nu)^{k} / 2^{k+1}, C_{\nu}\right)}{\log \left(\frac{2^{k+1}}{(1-\nu)^{k}}\right)}=\limsup _{k \rightarrow \infty} \frac{k \log 2}{k \log \left(\frac{2}{1-\nu}\right)+\log 2}
$$

Em particular, para $\nu=1 / 3$, obtemos o conjunto de Cantor tradicional e

$$
0=\operatorname{dim}_{\mathrm{T}}\left(C_{1 / 3}\right)<\operatorname{dim}_{\mathrm{H}}\left(C_{1 / 3}\right)=\operatorname{dim}_{\mathrm{F}}\left(C_{1 / 3}\right)=\frac{\log 2}{\log 3}<1
$$

Note que, dado um ponto $x \in X$, temos $\operatorname{dim}_{\mathrm{F}}(\{x\})=0$, pois $N(r,\{x\})=1$, para todo $r>0$. No entanto, diferente da dimensão de Hausdorff, os conjuntos enumeráveis não necessariamente herdam essa propriedade. De fato, a dimensão fractal é sensível a conjuntos enumeráveis, o que pode ser entendido como uma certa instabilidade desta dimensão. 
Exemplo 2.37. Os compactos associados às sequências $(1 / n)_{n}$ e $\left(1 / 2^{n}\right)_{n}$ possuem dimensão fractal $1 / 2$ e 0 , respectivamente.

Sejam $A=\{1 / n: n \in \mathbb{N}\} \cup\{0\}$ e $B=\left\{1 / 2^{n}: n \in \mathbb{N}\right\} \cup\{0\}$.

Dado $0<r<1$, tome $n=n(r) \geqslant 2$ tal que $\frac{1}{n(n+1)} \leqslant r<\frac{1}{(n-1) n}$. Os $n$ pontos de $\{1,1 / 2, \ldots, 1 / n\}$ estão separados uns dos outros pela distância mínima de $\frac{1}{(n-1) n}$, de modo que demandam pelo menos $n$ intervalos de tamanho $r$ para ser coberto, ou seja, $n \leqslant N(r,\{1,1 / 2, \ldots, 1 / n\}) \leqslant N(r, A)$.

Por outro lado, o restante de $A$, i.e., o conjunto $\left\{\frac{1}{n+1}, \frac{1}{n+2}, \ldots\right\} \cup\{0\}$, pode ser coberto pelos $n$ intervalos $[j r,(j+1) r]$ de comprimento $r$, com $j=0,1, \ldots, n-1$. Com isso, $n \leqslant N(r, A) \leqslant 2 n$ e $\frac{1}{(n+1)^{2}} \leqslant r<\frac{1}{(n-1)^{2}}$ implicam que

$$
1 / 2=\liminf _{n \rightarrow \infty} \frac{\log n}{\log (n+1)^{2}} \leqslant \operatorname{dim}_{\mathrm{F}}(A)=\limsup _{r \rightarrow 0^{+}} \frac{\log N(r, A)}{\log 1 / r} \leqslant \limsup _{n \rightarrow \infty} \frac{\log 2 n}{\log (n-1)^{2}}=1 / 2
$$

Quanto a $B$, basta proceder analogamente para obter

$$
\operatorname{dim}_{\mathrm{F}}(B)=\limsup _{r \rightarrow 0^{+}} \frac{\log N(r, B)}{\log 1 / r} \leqslant \limsup _{n \rightarrow \infty} \frac{\log 2 n}{\log 2^{n}}=0 .
$$

Exemplo 2.38. Vamos construir um conjunto $K \subset l^{2}$ compacto enumerável de dimensão fractal infinita, o qual, em particular, tem dimensão de Hausdorff zero, uma vez que é enumerável. (Mañé [24])

PASSO 1: para cada $m \in \mathbb{N}$, existe $K_{m} \subset \mathbb{R}^{m}$ compacto enumerável com $\operatorname{dim}_{\mathrm{F}}\left(K_{m}\right)=m$.

Fixado $m \in \mathbb{N}$, seja $\left(Q_{n}\right)_{n}$ uma sequência de cubos em $\mathbb{R}^{m}$ cujos lados medem $1 / 2^{n} \mathrm{e}$ tal que

$$
\mathrm{d}\left(Q_{n}, Q_{n+j}\right) \geqslant 1 / 2^{n}
$$

para todos os naturais $n$ e $j$; e

$$
\lim _{n \rightarrow \infty} \sup _{y \in Q_{n}}\|y\|_{\mathbb{R}^{m}}=0
$$

Note que por (2.3.2), podemos assumir que todos os cubos $Q_{n}$ caem na bola $B_{\mathbb{R}^{m}}(0,1 / m)$. 
Para cada $n$, tome pontos $x_{i}^{(n)} \in Q_{n}$ vértices da malha de comprimento $\left(1 / 2^{n}\right)^{n}$ contida em $Q_{n}$, de modo que $i$ varia de 1 a $\left(\left(1 / 2^{n}\right) /\left(1 / 2^{n}\right)^{n}\right)^{m}=2^{n(n-1) m}$.

Sendo assim, defina

$$
K_{m}=\{0\} \cup\left(\bigcup_{n=1}^{\infty}\left\{x_{i}^{(n)}: 1 \leqslant i \leqslant 2^{n(n-1) m}\right\}\right)
$$

de modo que $K_{m} \subset B_{\mathbb{R}^{m}}(0,1 / m)$ é um compacto enumerável.

De fato, seja $\left(x_{k}\right)_{k} \subset K_{m}$ tal que $x_{k} \rightarrow x \in \mathbb{R}^{m}$, com $k \rightarrow \infty$. A menos de passar a uma subsequência, podemos assumir que cada $x_{k}$ pertence a um cubo $Q_{n_{k}}$ diferente, então

$$
\|x\|_{\mathbb{R}^{m}}=\lim _{k \rightarrow \infty}\left\|x_{k}\right\|_{\mathbb{R}^{m}} \leqslant \lim _{k \rightarrow \infty} \sup _{y \in Q_{n_{k}}}\|y\|_{\mathbb{R}^{m}}=0
$$

daí $x=0 \in K_{m}$, o que implica que $K_{m}$ é fechado.

Note que, para cada $n$, tem-se $N\left(\left(1 / 2^{n}\right)^{n} / 2, K_{m}\right) \geqslant 2^{n(n-1) m}$, pois existe uma quantidade $2^{n(n-1) m}$ de pontos $x_{i}^{(n)} \in K_{m}$ e cada um deles demanda uma bola de raio $\left(1 / 2^{n}\right)^{n} / 2$. Assim,

$$
\operatorname{dim}_{\mathrm{F}}\left(K_{m}\right)=\limsup _{n \rightarrow \infty} \frac{\log N\left(\left(1 / 2^{n}\right)^{n} / 2, K_{m}\right)}{-\log \left(\left(1 / 2^{n}\right)^{n} / 2\right)} \geqslant \limsup _{n \rightarrow \infty} \frac{m \log \left(2^{n(n-1)}\right)}{-\log \left(\left(1 / 2^{n}\right)^{n} / 2\right)}=m
$$

consequentemente, $\operatorname{dim}_{\mathrm{F}}\left(K_{m}\right)=m$.

PASSO 2: Construção dos conjuntos em $l^{2}$.

Como $\mathbb{R}^{m}$ pode ser isometricamente imerso em $l^{2}$, considere os conjuntos $K_{m}$ construídos acima como subconjuntos de $l^{2}$ e seja

$$
K=\{0\} \cup\left(\bigcup_{m=1}^{\infty} K_{m}\right) \subset l^{2}
$$

de modo que $K$ é compacto enumerável e

$$
\operatorname{dim}_{\mathrm{F}}(K) \geqslant \sup _{m \in \mathbb{N}} \operatorname{dim}_{\mathrm{F}}\left(K_{m}\right)=\infty
$$

Para verificar que $K$ é compacto, tome $\left(u_{k}\right)_{k} \subset K \backslash\{0\}$. Se $\left(u_{k}\right)_{k}$ está numa reunião finita dos $K_{m}$, então, pela compacidade desta reunião finita, existe $u \in K$ limite de alguma subsequência de $\left(u_{k}\right)_{k}$. Caso contrário, podemos passar a uma subsequência de tal forma 
que cada $u_{k_{j}}$ pertença a um $K_{m_{j}}$ diferente $\left(\operatorname{com}\left(m_{j}\right)_{j}\right.$ divergente), e então $\left(u_{k_{j}}\right)_{j}$ converge para $u=0 \in K$, pois por construção tem-se $\left\|u_{k_{j}}\right\|_{l^{2}}<1 / m_{j}$.

A seguinte propriedade é um dos motivos pelos quais a dimensão fractal é preferida à de Hausdorff.

Lema 2.39. Sejam $K_{1}$ e $K_{2}$ compactos de um espaço vetorial, então

$$
\operatorname{dim}_{\mathrm{F}}\left(K_{1}+K_{2}\right) \leqslant \operatorname{dim}_{\mathrm{F}}\left(K_{1}\right)+\operatorname{dim}_{\mathrm{F}}\left(K_{2}\right)
$$

Demonstração: Pondo $N_{i}=N\left(r, K_{i}\right)$, existem $x_{1}^{i}, \ldots, x_{N_{i}}^{i} \in K_{i}$ de modo que $K_{i} \subset$ $\bigcup_{j=1}^{N_{i}} B_{X}\left(x_{j}^{i}, r\right)$, para $i=1$ e $i=2$, e então

$$
K_{1}+K_{2} \subset \bigcup_{i=1}^{N_{2}} \bigcup_{j=1}^{N_{1}}\left(B_{X}\left(x_{j}^{1}, r\right)+B_{X}\left(x_{i}^{2}, r\right)\right)
$$

Como $B_{X}\left(x_{j}^{1}, r\right)+B_{X}\left(x_{i}^{2}, r\right)=B_{X}\left(x_{j}^{1}+x_{i}^{2}, 2 r\right)$, segue que $N\left(2 r, K_{1}+K_{2}\right) \leqslant N_{1} N_{2}=$ $N\left(r, K_{1}\right) N\left(r, K_{2}\right)$, e assim,

$$
\begin{aligned}
\operatorname{dim}_{\mathrm{F}}\left(K_{1}+K_{2}\right) & =\limsup _{r \rightarrow 0^{+}} \frac{\log N\left(2 r, K_{1}+K_{2}\right)}{\log 1 / 2 r} \\
& \leqslant \limsup _{r \rightarrow 0^{+}} \frac{\log N\left(r, K_{1}\right) N\left(r, K_{2}\right)}{\log 1 / 2 r} \\
& =\limsup _{r \rightarrow 0^{+}} \frac{\log N\left(r, K_{1}\right)}{\log 1 / 2 r}+\limsup _{r \rightarrow 0^{+}} \frac{\log N\left(r, K_{2}\right)}{\log 1 / 2 r} \\
& =\limsup _{r \rightarrow 0^{+}} \frac{\log N\left(r, K_{1}\right)}{\log 1 / 2+\log 1 / r}+\limsup _{r \rightarrow 0^{+}} \frac{\log N\left(r, K_{2}\right)}{\log 1 / 2+\log 1 / r} \\
& =\limsup _{r \rightarrow 0^{+}} \frac{\log N\left(r, K_{1}\right)}{\log 1 / r} \frac{1}{\log N\left(r, K_{2}\right)} \frac{1}{\log 1 / 2}+\limsup _{r \rightarrow 0^{+}} \frac{1 / r}{\log 1 / r} \\
& =\operatorname{dim}_{\mathrm{F}}\left(K_{1}\right)+\operatorname{dim}_{\mathrm{F}}\left(K_{2}\right) .
\end{aligned}
$$

Corolário 2.40. Sejam $X$ um espaço vetorial normado e $K \subset X$ um compacto tal que $\operatorname{dim}_{\mathrm{F}}(K)<\infty$, então $\operatorname{dim}_{\mathrm{F}}(K-K) \leqslant 2 \operatorname{dim}_{\mathrm{F}}(K)$.

O conteúdo que apresentamos pode ser encontrado em Carvalho [8], Edgar [12], Falconer [16], Falconer [17], Mañé [24], Pesin [26] e Robinson [27]. 


\subsection{Um Comparativo}

Quando trabalhamos com espaços métricos compactos $X$, faz sentido se perguntar que possível relação existe, se existe, entre estas três noções de dimensão.

Esta seção se dedica a mostrar que

$$
\operatorname{dim}_{\mathrm{T}}(X) \leqslant \operatorname{dim}_{\mathrm{H}}(X) \leqslant \operatorname{dim}_{\mathrm{F}}(X)
$$

para espaços métrico compactos $X$. Esta cadeia de desigualdade faz com que uma dimensão eventualmente herde propriedades de outra. Em especial, se a dimensão fractal for finita, então a topológica também e isto será de grande importância, dados os Teoremas de Imersão e de Mañé.

Teorema 2.41. Se $X$ é um espaço métrico compacto, então

$$
\operatorname{dim}_{\mathrm{H}}(X) \leqslant \operatorname{dim}_{\mathrm{F}}(X)
$$

\section{Demonstração:}

Podemos assumir que $\operatorname{dim}_{\mathrm{F}}(X)$ é finita, pois a desigualdade é trivialmente verdadeira se $\operatorname{dim}_{\mathrm{F}}(X)=\infty$.

Fixado $r>0$, sabemos que $X$ pode ser coberto por bolas abertas $B_{1}, \ldots, B_{N(r, X)}$ de raio $r$, de modo que, para cada $\alpha>0$, vale

$$
\mu_{2 r}^{\alpha}(X) \leqslant \sum_{j=1}^{N(r, X)}\left(\operatorname{diam}\left(B_{j}\right)\right)^{\alpha}=N(r, X)(2 r)^{\alpha}
$$

Agora, dado $\eta>0$, sejam $\alpha=\operatorname{dim}_{\mathrm{F}}(X)+\eta$ e $\varepsilon \in(0, \eta)$. Para algum $\delta>0$ e todo $0<r<\delta$ vale

$$
\begin{aligned}
\mu_{2 r}^{\left(\operatorname{dim}_{\mathrm{F}}(X)+\eta\right)}(X) & \leqslant N(r, X)(2 r)^{\operatorname{dim}_{\mathrm{F}}(X)+\eta} \\
& \leqslant\left(\frac{1}{r}\right)^{\operatorname{dim}_{\mathrm{F}}(X)+\varepsilon}(2 r)^{\operatorname{dim}_{\mathrm{F}}(X)+\eta} \\
& =2^{\operatorname{dim}_{\mathrm{F}}(X)+\eta} r^{\eta-\varepsilon}
\end{aligned}
$$

e como $\eta-\varepsilon>0$, ao passar o limite com $r \rightarrow 0^{+}$, obtemos $\mu^{\left(\operatorname{dim}_{\mathrm{F}}(X)+\eta\right)}(X)=0$. 
Da definição de $\operatorname{dim}_{H}(X)$, resulta que $\operatorname{dim}_{\mathrm{H}}(X) \leqslant \operatorname{dim}_{\mathrm{F}}(X)+\eta$, em que $\eta>0$ é arbitrário.

Não vale a igualdade, em geral. Para muitos conjuntos "razoavelmente regulares" vale, como para bolas fechadas de $\mathbb{R}^{m}$, mas há vários exemplos de desigualdade estrita, como vimos na Seção 2.3. Em Pesin [26], Exemplo 6.1, vemos que, dado $0<\eta<1$, existe um compacto enumerável $K \subset[0,1]$ tal que $\operatorname{dim}_{F}(K)=\eta$; enquanto que, sabidamente, $\operatorname{dim}_{H}(K)=0$.

Provar que a dimensão de Hausdorff domina a dimensão topólogica, porém, é uma tarefa mais trabalhosa e vai demandar o conceito intermediário de dimensão indutiva e algumas generalizações da Teoria de Medida, os quais foram postos nos Apêndices B e C. respectivamente, para efeito de organização.

Seguindo a abordagem de Hurewicz \& Wallman [20], Edgar [11] e Edgar [12], e usando fortemente o fato de que ind $(X)=\operatorname{dim}_{\mathrm{T}}(X)$, tendo como referência Jan Van Mill [21], seguimos o roteiro abaixo:

1) definir a (pequena) dimensão indutiva, ind $(X)$, para espaços métricos separáveis;

2) $\operatorname{mostrar}$ que ind $(X) \leqslant \operatorname{dim}_{H}(X)$.

Em Robinson [27] (Teorema 2.11), prova-se de maneira sucinta que

$$
\operatorname{dim}_{\mathrm{T}}(X) \leqslant \operatorname{dim}_{\mathrm{H}}(X)
$$

quando $X$ é compacto. Esta abordagem evita recorrer aos tópicos de medida que vamos apresentar e difere completamente da argumentação proposta no clássico livro de Hurewicz \& Wallman [20], apesar de, com ajustes, se aplicar a espaços métricos separáveis; é, de fato, uma demonstração a parte. Estas são as duas principais razões para não adotá-la.

A cada espaço métrico separável $X=(X, \mathrm{~d})$, associamos uma quantidade, $\operatorname{ind}(X)$, que assume valor em $\{-1,0,1,2, \ldots\} \cup\{\infty\}$, a qual é chamada dimensão indutiva e, assim como sugere o nome, é dada recursivamente segundo a definição abaixo.

Definição 2.42. Definimos a dimensão indutiva de $X$, com a notação ind $(X)$, como sendo

1) $\operatorname{ind}(X)=-1$, se $X$ é vazio; 
2) $X$ não vazio tem dimensão indutiva finita menor ou igual a $m \in \mathbb{Z}_{+}$, indicando por $\operatorname{ind}(X) \leqslant m$, quando, quando todo ponto $x \in X$ admite vizinhanças $V$ arbitrariamente pequenas de modo que ind $(\partial V) \leqslant m-1$;

3) X tem pequena dimensão indutiva igual a $m$, indicando por $\operatorname{ind}(X)=m$, se $m$ é o menor inteiro positivo tal que ind $(X) \leqslant m$;

4) $X$ tem pequena dimensão indutiva infinita, e escrevemos ind $(X)=\infty$, se não existe $m$ tal que $\operatorname{ind}(X) \leqslant m$.

Tal denominação é um tanto quanto rara, pois é largamente conhecida como pequena dimensão indutiva (livre tradução de small inductive dimension), uma vez que também existe a grande dimensão indutiva (consulte o Apêndice B). No entanto, para os propósitos deste texto, a importância da pequena é maior que a da grande dimensão indutiva. Pode ser ainda encontrada sob a denominação de weak inductive dimension ou Urysohn-Menger dimension.

Os dois lemas que apresentamos estabelecem a maneira pela qual ind $(X)$ e $\operatorname{dim}_{\mathrm{H}}(X)$ serão usadas. Dados $x_{0} \in X$ e $r>0$, denotamos a bola aberta de $X$ centrada em $x_{0}$ de raio $r$ por

$$
B_{X}\left(x_{0}, r\right)=\left\{x \in X: \mathrm{d}\left(x, x_{0}\right)<r\right\}
$$

e a esfera de $X$ centrada em $x_{0}$ de raio $r$ por

$$
S_{X}\left(x_{0}, r\right)=\left\{x \in X: \mathrm{d}\left(x, x_{0}\right)=r\right\} .
$$

Lema 2.43. Se $X$ é um espaço métrico separável tal que ind $(X)>n$, então existem $x_{0} \in X$ e $r>0$ de modo que

$$
\operatorname{ind}\left(S_{X}\left(x_{0}, \varepsilon\right)\right)>n-1
$$

para todo $0<\varepsilon<r$.

\section{Demonstração:}

Por hipótese, não é verdade que ind $(X) \leqslant n$, daí existe $x_{0} \in X$ que não admite vizinhanças arbitrariamente pequenas com a propriedade de que ind $(\partial V) \leqslant n-1$. Em 
particular, a coleção das bolas abertas centradas em $x_{0}, B_{X}\left(x_{0}, r\right)$, não pode satisfazer ind $\left(\partial B_{X}\left(x_{0}, r\right)\right) \leqslant n-1$ para $r>0$ arbitrariamente pequeno, de modo que existe $r>0$ tal que ind $\left(\partial B_{X}\left(x_{0}, \varepsilon\right)\right)>n-1$, para todo $0<\varepsilon<r$.

Como $\partial B_{X}\left(x_{0}, \varepsilon\right) \subset S_{X}\left(x_{0}, \varepsilon\right)$ e ind $(\cdot)$ é monótona, segue que

$$
\operatorname{ind}\left(S_{X}\left(x_{0}, \varepsilon\right)\right) \geq \operatorname{ind}\left(\partial B_{X}\left(x_{0}, \varepsilon\right)\right)>n-1,
$$

para tais valores de $\varepsilon$.

Lema 2.44. Seja $X$ um espaço métrico separável tal que $\mu^{(\alpha+1)}(X)=0$.

Para cada $x_{0} \in X$, vale

$$
\mu^{(\alpha)}\left(S_{X}\left(x_{0}, r\right)\right)=0 \text { q.s. }
$$

Em particular, o conjunto de tais $r>0$ é denso em $(0, \infty)$.

\section{Demonstração:}

Dado $A \subset X$, se $r_{1}=\inf _{x \in A} \mathrm{~d}\left(x, x_{0}\right)$ e $r_{2}=\sup _{x \in A} \mathrm{~d}\left(x, x_{0}\right)$, então $r_{2}-r_{1} \leqslant \operatorname{diam}(A)$. Assim, tomando a integral superior para a função

$$
[0, \infty) \ni r \longmapsto \operatorname{diam}\left(A \cap S_{X}\left(x_{0}, r\right)\right) \in[0, \infty),
$$

a qual não é necessariamente mensurável, temos

$$
\begin{aligned}
\bar{\int}_{[0, \infty)}\left(\operatorname{diam}\left(A \cap S_{X}\left(x_{0}, r\right)\right)\right)^{\alpha} d r & =\bar{\int}_{\left(r_{1}, r_{2}\right)}\left(\operatorname{diam}\left(A \cap S_{X}\left(x_{0}, r\right)\right)\right)^{\alpha} d r \\
& \leqslant(\operatorname{diam}(A))^{\alpha} \int_{\left(r_{1}, r_{2}\right)} d r \\
& \leqslant(\operatorname{diam}(A))^{\alpha+1}
\end{aligned}
$$

Uma vez que $0=\mu^{(\alpha+1)}(X)=\sup _{\delta>0} \mu_{\delta}^{(\alpha+1)}(X)$, obtemos, para cada $n \in \mathbb{N}$, uma cobertura $\left\{A_{j}^{n}\right\}_{j \in \mathbb{N}}$ de $X$ de modo que $\operatorname{diam}\left(A_{j}^{n}\right) \leqslant 1 / 2^{n} \mathrm{e}$

$$
\sum_{j=1}^{\infty}\left(\operatorname{diam}\left(A_{j}^{n}\right)\right)^{\alpha+1} \leqslant 1 / 2^{n} .
$$


Por (2.4.1) acima, obtemos

$$
\sum_{j=1}^{\infty} \bar{\int}_{[0, \infty)}\left(\operatorname{diam}\left(A_{j}^{n} \cap S_{X}\left(x_{0}, r\right)\right)\right)^{\alpha} d r \leqslant 1 / 2^{n}
$$

e então pelo Teorema C.6

$$
\bar{\int}_{[0, \infty)} f_{n}(r) d r \leqslant 1 / 2^{n}
$$

em que $f_{n}(r)=\sum_{j=1}^{\infty}\left(\operatorname{diam}\left(A_{j}^{n} \cap S_{X}\left(x_{0}, r\right)\right)\right)^{\alpha}$ e isto implica que $f_{n}(r) \rightarrow 0$ q.s.

De fato, seja $E_{n}=\left\{r \in[0, \infty): f_{n}(r) \geqslant 1 / n\right\}$, daí

$$
1 / 2^{n} \geqslant \int_{E_{n}} f_{n}(r) d r \geqslant \frac{1}{n} \bar{m}\left(E_{n}\right)
$$

de modo que

$$
\bar{m}\left(\bigcup_{n=N}^{\infty} E_{n}\right) \leqslant \sum_{n=N}^{\infty} \bar{m}\left(E_{n}\right) \leqslant \sum_{n=N}^{\infty} \frac{n}{2^{n}} \stackrel{N \rightarrow \infty}{\longrightarrow} 0 .
$$

Se $r$ é tal que $f_{n}(r) \not \rightarrow 0$, então existe uma infinidade de índices $n$ tais que $f_{n}(r)>1 / n$. E, uma vez que $E_{1} \subset E_{2} \subset E_{3} \ldots$, segue que

$$
\left\{r \in[0, \infty): f_{n}(r) \not \neg 0\right\} \subset \bigcup_{n=N}^{\infty} E_{n}
$$

para todo $N$; consequentemente

$$
\lim _{n \rightarrow \infty} \sum_{j=1}^{\infty}\left(\operatorname{diam}\left(A_{j}^{n} \cap S_{X}\left(x_{0}, r\right)\right)\right)^{\alpha}=\lim _{n \rightarrow \infty} f_{n}(r)=0
$$

para quase todo $r \geqslant 0$, no sentido de Lebesgue.

Como as coleções $\left\{A_{j}^{n} \cap S_{X}\left(x_{0}, r\right)\right\}_{j \in \mathbb{N}}$ são $\left(1 / 2^{n}\right)$-coberturas de $S_{X}\left(x_{0}, r\right)$, concluímos que $\mu^{(\alpha)}\left(S_{X}\left(x_{0}, r\right)\right)=0$ q.s., por definição de $\alpha$-medida de Hausdorff.

Basta observar que, para quase todo $r$,

$$
\liminf _{n} \mu_{1 / 2^{n}}^{(\alpha)}\left(S_{X}\left(x_{0}, r\right)\right) \leqslant \limsup _{n} \mu_{1 / 2^{n}}^{(\alpha)}\left(S_{X}\left(x_{0}, r\right)\right) \leqslant \limsup _{n} 1 / 2^{n}=0
$$


Comentamos não ser verdade, em geral, que

$$
[0, \infty) \ni r \longmapsto \operatorname{diam}\left(A \cap S_{X}\left(x_{0}, r\right)\right) \in[0, \infty)
$$

é uma função mensurável. De fato, mesmo que $A \subset X$ fosse aberto limitado, ainda não seria possível garantir a mensurabilidade da aplicação.

Sejam $E \subset(0,1]$ um conjunto não mensurável e $X=[-1,0] \cup E$, o qual é um espaço métrico com a métrica usual de $\mathbb{R}$. Pondo $x_{0}=0$ e $A=X$ (aberto e limitado), temos que $A \cap S_{X}(0, r)=S_{X}(0, r)$ consiste dos pontos $-r$ e $r$, quando $r \in E$, e é unitário $\{-r\}$, caso contrário. Com isso,

$$
\operatorname{diam}\left(A \cap S_{X}(0, r)\right)= \begin{cases}2 r, & \text { se } r \in E \\ 0, & \text { se } r \notin E\end{cases}
$$

ou seja, $\operatorname{diam}\left(A \cap S_{X}(0, r)\right)=2 r \chi_{E}(r)$, logo uma função não mensurável.

Em resumo, como a fronteira de $B_{X}\left(x_{0}, r\right)$ está contida em $S_{X}\left(x_{0}, r\right)$, o Lema 2.44 nos diz que se um espaço métrico separável $X$ tem $(\alpha+1)$-medida de Hausdorff igual a zero, então um ponto qualquer deste espaço possui vizinhanças de diâmetro $2 r$ arbitrariamente pequeno cujas fronteiras tem $\alpha$-medida de Hausdorff igual a zero. Esta propriedade será invocada de maneira indutiva para mostrar que ind $(X) \leqslant \operatorname{dim}_{\mathrm{H}}(X)$.

Teorema 2.45. Se $X$ é um espaço métrico separável tal que $\mu^{(n+1)}(X)=0$, para algum inteiro $n+1 \geqslant 0$, então $\operatorname{ind}(X) \leqslant n$.

Consequentemente,

$$
\operatorname{dim}_{\mathrm{T}}(X)=\operatorname{ind}(X) \leqslant \operatorname{dim}_{\mathrm{H}}(X) .
$$

\section{Demonstração:}

Basta mostrar que $X$ tem dimensão indutiva menor ou igual a $n$ em $x_{0} \in X$ arbitrário. Vamos proceder por indução. Para $n=-1$,

$$
0=\mu^{(0)}(X)=\sup _{\delta>0}\left(\inf \left\{\sum_{j=1}^{\infty}\left(\operatorname{diam}\left(A_{j}\right)\right)^{0}: X \subset \bigcup_{j=1}^{\infty} A_{j}, \operatorname{diam}\left(A_{j}\right)<\delta\right\}\right),
$$

implica que $X=\varnothing$ e $\operatorname{assim} \operatorname{ind}(X)=-1$.

Suponha por indução que o resultado vale para $n$, i.e., suponha que todo espaço métrico com $n$-medida de Hausdorff nula tem dimensão indutiva menor ou igual a $n-1$. Assuma 
que $\mu^{(k+1)}(X)=0$.

Seja $x_{0} \in X$. Pelo Lema 2.44 ,

$$
\mu^{(k)}\left(S_{X}\left(x_{0}, r\right)\right)=0
$$

para $r \geqslant 0$ arbitrariamente pequeno.

Claro que $S_{X}\left(x_{0}, r\right)=\left(S_{X}\left(x_{0}, r\right), \mathrm{d}\right)$ é um espaço métrico e lhe aplicando a hipótese de indução, temos que ind $\left(S_{X}\left(x_{0}, r\right)\right) \leqslant k-1$, para tais $r$.

Em outras palavras,

$$
\operatorname{ind}\left(S_{X}\left(x_{0}, r\right)\right) \leqslant k-1
$$

para valores de $r$ arbitrariamente pequenos; a contrapositiva do Lema 2.43 então implica que ind $(X) \leqslant k$.

Se $\operatorname{dim}_{H}(X)=\infty$, não há o que provar. Por outro lado, seja $n_{0}$ inteiro tal que $\operatorname{dim}_{\mathrm{H}}(X) \in\left[n_{0}, n_{0}+1\right)$. Como $\mu^{\left(n_{0}+1\right)}(X)=0$, segue que

$$
\operatorname{ind}(X) \leqslant n_{0} \leqslant \operatorname{dim}_{\mathrm{H}}(X)
$$

Por fim, o Teorema B.9 (que será demonstrado no Apêndice B) afirma que a dimensão topológica e a dimensão indutiva coincidem, de onde concluímos que

$$
\operatorname{dim}_{\mathrm{T}}(X)=\operatorname{ind}(X) \leqslant \operatorname{dim}_{\mathrm{H}}(X)
$$

como queríamos.

Corolário 2.46. Se X é um espaço métrico compacto, então

$$
\operatorname{dim}_{\mathrm{T}}(X) \leqslant \operatorname{dim}_{\mathrm{H}}(X) \leqslant \operatorname{dim}_{\mathrm{F}}(X)
$$

\section{Demonstração:}

É imediato dos Teoremas 2.41 e 2.45. 


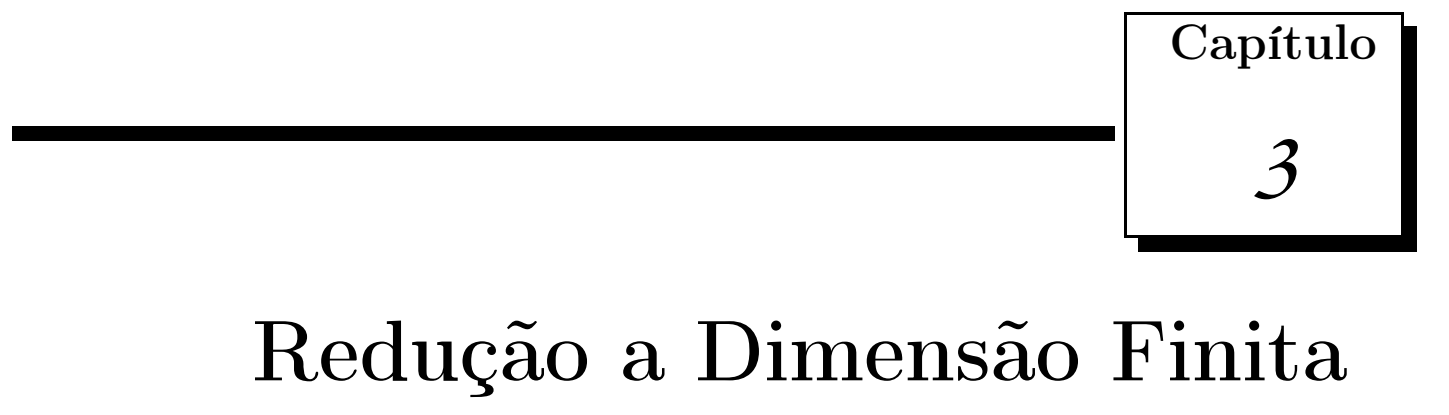

O atrator global de um semigrupo em $X$, como vimos, é a reunião de todas as órbitas limitadas, ou equivalentemente, é a reunião de todos os $\omega$-limite de conjuntos limitados de $X$, o que dita de que forma os pontos do espaço evoluem pela ação do semigrupo. Ele é o objeto que, de fato, concentra toda a informação sobre a dinâmica assintótica.

Podemos descrever com precisão ímpar a dinâmica dos semigrupos gradientes. No entanto, não é de se esperar que isso sempre ocorra, o que nos traz à pergunta: como lidar com esses objetos, usualmente de espaços de Banach, e extrair as informações dinâmicas que contém?

A resposta não é imediata, mas existe um meio que facilita a busca por respostas: se pudermos "enxergar" o atrator global sob a perspectiva de algum espaço euclidiano finito dimensional, então conquistamos todos os benefícios de se trabalhar em dimensão finita, entre eles a análise topológica e a intuição geométrica de órbitas que vemos em $\mathbb{R}^{m}$. Claro que devemos ter a certeza de que informações não são perdidas quando reduzimos o problema a dimensão finita. Esta abordagem tem movimentado a área nos últimos anos.

Este capítulo considera os dois resultados que nos permitem efetuar a redução a dimensão finita: o Teorema da Imersão e o Teorema de Mañé. O primeiro garante a imersão de espaços métricos compactos de dimensão topológica $m$ em $\mathbb{R}^{2 m+1}$ e detém a essência e a inspiração para o Teorema de Mañé, segundo o qual subconjuntos $K \sigma$-compactos de espaços de Banach tais que $\operatorname{dim}_{H}(K-K)$ é finita podem ser projetados injetivamente em algum subespaço de dimensão finita. Ambos são aplicações do Teorema da Categoria de Baire por meio de apropriados abertos densos no espaço das aplicações contínuas. 


\subsection{O Teorema da Imersão}

O principal resultado desta seção, atribuído a K. Menger e G. Nöbeling, afirma que todo espaço métrico compacto de dimensão topológica $m$ pode ser imerso em $\mathbb{R}^{2 m+1}$. Sua demonstração é uma aplicação do Teorema da Categoria de Baire e generaliza o Teorema de Whitney, segundo o qual toda $m$-variedade compacta pode ser imersa em $\mathbb{R}^{N}$, para algum $N$. Além disso, sua essência é o que norteia a demonstração do Teorema de Mañé, o qual nos permitirá, em contextos ainda bem gerais, projetar injetivamente um atrator global em $\mathbb{R}^{N}$, para algum $N$, mesmo que o espaço de Banach no qual o semigrupo está definido seja infinito dimensional.

Em Hurewicz \& Wallman [20] e Jan van Mill [21], o Teorema da Imersão é abordado para espaços métricos separáveis; no entanto, vamos proceder com a sua versão para espaços métricos compactos, que são os de aplicabilidade imediata. Com isso, seguimos Munkres 25]

Está longe de óbvio o porquê de $2 m+1$ ser a dimensão crucial para se obter a imersão procurada. Mas é a geometria de $\mathbb{R}^{N}$ que se encarrega de explicar isso, como veremos.

Um conjunto $\left\{z_{0}, \ldots, z_{k}\right\} \subset \mathbb{R}^{m}$ é dito geometricamente independente, ou abreviadamente G.I., quando

$$
\sum_{j=0}^{k} a_{j} z_{j}=0 \text { e } \sum_{j=0}^{k} a_{j}=0
$$

implicarem $a_{0}=\ldots=a_{k}=0$.

Nestas condições, como $a_{0}=-\sum_{j=1}^{k} a_{j}$, dizer que $\left\{z_{0}, \ldots, z_{k}\right\}$ é G.I. é dizer que

$$
\sum_{j=1}^{k} a_{j}\left(z_{j}-z_{0}\right)=0
$$

implica $a_{1}=\ldots=a_{k}=0$ e consequentemente $a_{0}=0$. Ou seja, $\left\{z_{0}, \ldots, z_{k}\right\}$ é G.I. se, e somente se, $z_{1}-z_{0}, \ldots, z_{k}-z_{0}$ são linearmente independentes em $\mathbb{R}^{m}$.

Há uma lição a ser tirada dessa equivalência: dois pontos distintos sempre formam um conjunto G.I.; quando não colineares, três pontos o fazem; quando não coplanares, quatro pontos de $\mathbb{R}^{3}$ também; e assim por diante. E ainda, qualquer conjunto G.I. de pontos de $\mathbb{R}^{m}$ contém no máximo $m+1$ pontos.

Um conjunto de $\mathbb{R}^{m}$ está em posição geral em $\mathbb{R}^{m}$ quando todo seu subconjunto 
contendo no máximo $m+1$ pontos é G.I..

O próximo lema nos diz que, dado um conjunto $\left\{z_{1}, \ldots, z_{k}\right\} \subset \mathbb{R}^{m}$, não necessariamente em posição geral em $\mathbb{R}^{m}$, podemos "mover" estes pontos ligeiramente de modo que o conjunto resultante sim esteja em posição geral em $\mathbb{R}^{m}$.

Lema 3.1. Dados $\varepsilon>0$ e $\left\{z_{1}, \ldots, z_{k}\right\} \subset \mathbb{R}^{m}$, existe um conjunto $\left\{y_{1}, \ldots, y_{k}\right\} \subset \mathbb{R}^{m}$ que está em posição geral em $\mathbb{R}^{m}$ de modo que

$$
\left\|z_{j}-y_{j}\right\|<\varepsilon, \quad j=1, \ldots, k .
$$

Demonstração: Vamos proceder por indução.

Seja $y_{1}:=z_{1}$. Suponha $y_{1}, \ldots, y_{p}$ em posição geral em $\mathbb{R}^{m}$ tais que

$$
\left\|z_{j}-y_{j}\right\|<\varepsilon, j=1, \ldots, p \text {. }
$$

Cada subconjunto de $\left\{y_{1}, \ldots, y_{p}\right\}$ com no máximo $m$ pontos é G.I. e determina um $l$-plano em $\mathbb{R}^{m}$, com $l \leqslant m-1, \operatorname{logo}$ de interior vazio em $\mathbb{R}^{m}$. Uma vez que existe uma quantidade finita destes planos, sua união também tem interior vazio, pelo Teorema da Categoria de Baire. Seja então $y_{p+1} \in B_{\mathbb{R}^{m}}\left(z_{p+1}, \varepsilon\right)$ de modo que não esteja na união destes planos.

Afirmo que $C=\left\{y_{1}, \ldots, y_{p}, y_{p+1}\right\}$ está em posição geral em $\mathbb{R}^{m}$.

Sabemos que $y_{p}-y_{1}, \ldots, y_{2}-y_{1}$ são linearmente independente. Se $C$ não estivesse em posição geral em $\mathbb{R}^{m}$, então $y_{p+1}-y_{1}, y_{p}-y_{1}, \ldots, y_{2}-y_{1}$ seriam linearmente dependente, o que contraria a escolha de $y_{p+1}$.

E isto encerra a demonstração.

Para clarear as ideias, vamos dar um esboço da demonstração do Teorema da Imersão antes de fazê-la.

Considere os espaços de Banach:

- $\mathbb{R}_{\infty}^{N}$, o espaço $\mathbb{R}^{N}$ munido da norma $\|x\|_{\infty}=\max _{1 \leqslant j \leqslant N}\left|x_{j}\right|$

- $C\left(X, \mathbb{R}_{\infty}^{N}\right)$, o espaço das funções contínuas munido da norma $\|f\|=\max _{x \in X}\|f(x)\|_{\infty}$. 
Dada $f \in C\left(X, \mathbb{R}_{\infty}^{N}\right)$, defina

$$
\Delta(f):=\sup _{z \in f(X)} \operatorname{diam}\left(f^{-1}(\{z\})\right)
$$

de modo que

$$
\Delta(f)=0 \Leftrightarrow f \text { é injetora, }
$$

i.e., $\Delta(f)$ é uma medida do quanto a função $f$ deixa de ser injetora.

Como é sabido, estamos à procura de uma função $f \in C\left(X, \mathbb{R}_{\infty}^{N}\right)$ que seja de fato um homeomorfismo sobre sua imagem. Claro, $f$ é injetora. Isto nos sugere usar a medida $\Delta$ acima a nosso favor. É basicamente o que é feito.

Vamos proceder da seguinte maneira:

1) vamos definir $\mathcal{U}_{\delta}$, o conjunto das funções $f$ tais que seu "grau" de não injetividade é menor que $\delta$;

2) a partir da compacidade de $X$, mostramos que $\mathcal{U}_{\delta}$ é um aberto de $C\left(X, \mathbb{R}_{\infty}^{N}\right)$;

3) para mostrar que $\mathcal{U}_{\delta}$ é também denso, dada $f \in C\left(X, \mathbb{R}_{\infty}^{N}\right)$, definimos uma função $g \in \mathcal{U}_{\delta}$ tal que $\|g-f\|<\varepsilon$; sua construção é graças a uma partição da unidade associada a uma conveniente cobertura aberta de $X$ com ordem $m+1$. Esta é a etapa mais longa e que invoca as propriedades geométricas de $\mathbb{R}^{N}$, o fato de $\operatorname{dim}_{\mathrm{T}}(X)=m$ e novamente a compacidade de $X$;

4) do Teorema da Categoria de Baire, conseguimos que $\bigcap_{n=1}^{\infty} \mathcal{U}_{1 / n} \operatorname{denso} \operatorname{em} C\left(X, \mathbb{R}_{\infty}^{N}\right)$;

5) a existência de $f: X \rightarrow \mathbb{R}_{\infty}^{N}$, aplicação contínua injetora e fechada, era exatamente o que se buscava.

Estabelecido o roteiro, vamos à demonstração!

\section{Teorema 3.2. (Teorema da Imersão)}

Todo espaço métrico compacto $X$ de dimensão topológica m pode ser imerso em $\mathbb{R}^{2 m+1}$.

\section{Demonstração:}

Defina $\Delta(f):=\sup _{z \in f(X)} \operatorname{diam}\left(f^{-1}(\{z\})\right)$, para cada $f \in C\left(X, \mathbb{R}_{\infty}^{N}\right)$, e

$$
\mathcal{U}_{\delta}=\left\{f \in C\left(X, \mathbb{R}_{\infty}^{N}\right): \Delta(f)<\delta\right\}
$$

para cada $\delta>0$. Não é imposto que $N=2 m+1$; esta relação será conquistada. 
Mostremos que $\mathcal{U}_{\delta}$ é aberto denso em $C\left(X, \mathbb{R}_{\infty}^{N}\right)$, para todo $\delta>0$. Do Teorema da Categoria de Baire, teremos que $\bigcap_{n=1}^{\infty} \mathcal{U}_{1 / n}$ é denso em $C\left(X, \mathbb{R}_{\infty}^{N}\right)$. Em outras palavras, a coleção das funções $f \in C\left(X, \mathbb{R}_{\infty}^{N}\right)$ injetoras é um conjunto denso neste espaço; em particular, existe $f: X \rightarrow \mathbb{R}_{\infty}^{N}$ contínua injetora definida no compacto $X$. Logo, $f$ é uma imersão de $X$ em $\mathbb{R}_{\infty}^{N}$, e isto encerrará a demonstração.

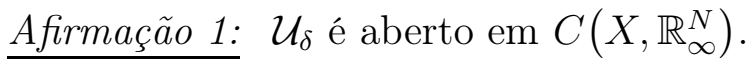

Dada $f \in \mathcal{U}_{\delta}$, queremos $\eta>0$ de modo que $B(f, \eta) \subset \mathcal{U}_{\delta}$ com a norma de $C\left(X, \mathbb{R}_{\infty}^{N}\right)$.

Tome $b$ tal que $\Delta(f)<b<\delta$ e note que $\mathrm{d}(x, y)<b$, sempre que $f(x)=z=f(y)$. Como a aplicação

$$
X \times X \ni(x, y) \longmapsto\|f(x)-f(y)\|_{\infty} \in \mathbb{R}^{+}
$$

é positiva no compacto $F=\{(x, y) \in X \times X: \mathrm{d}(x, y) \geqslant b\}$, segue que ela admite um mínimo positivo em $F$, digamos $2 \eta>0$.

Seja $g \in B(f, \eta)$. Assim, para todo $(x, y) \in F$, tem-se

$$
\begin{aligned}
\|g(x)-g(y)\|_{\infty} & \geqslant\|f(x)-f(y)\|_{\infty}-\|g(y)-f(y)\|_{\infty}-\|f(x)-g(x)\|_{\infty} \\
& >2 \eta-\eta-\eta=0
\end{aligned}
$$

ou equivalentemente, se $g(x)=g(y)$, então $(x, y) \notin F$, i.e., $\mathrm{d}(x, y)<b$, e consequentemente $\Delta(g) \leqslant b<\delta$, i.e., $g \in \mathcal{U}_{\delta}$.

Logo, $B(f, \eta) \subset \mathcal{U}_{\delta}$, como queríamos.

Afirmação 2: cada $\mathcal{U}_{\delta}$ é denso em $C\left(X, \mathbb{R}_{\infty}^{N}\right)$.

Esta é a parte mais custosa e na qual vamos precisar dos tópicos de geometria analítica de $\mathbb{R}_{\infty}^{N}$ discutidos.

Sejam $\delta>0$ e $f \in C\left(X, \mathbb{R}_{\infty}^{N}\right)$. Dado $\varepsilon>0$, queremos encontrar $g \in \mathcal{U}_{\delta}$ tal que

$$
\sup _{x \in X}\|f(x)-g(x)\|_{\infty}<\varepsilon .
$$

Considere $\left\{U_{1}, \ldots, U_{n}\right\}$ uma cobertura aberta de $X$ com ordem $m+1$ de modo que

$$
\operatorname{diam} U_{j}<\delta / 2 \text { e } \operatorname{diam} f\left(U_{j}\right)<\varepsilon / 2
$$


o que é possível pois $X$ é compacto de dimensão $m$ e $f$ é contínua de $X$ em $\mathbb{R}_{\infty}^{N}$. De fato, da continuidade de $f$, para cada $x \in X$, tome $U_{x} \subset X$ uma vizinhança de $x$ tal que diam $U_{x}<\delta / 2$ e $f\left(U_{x}\right) \subset B_{\mathbb{R}_{\infty}^{N}}(f(x), \varepsilon / 2)$. De $\left\{U_{x}\right\}_{x \in X}$ extraia um refinamento com ordem $m+1$ e agora usando a compacidade de $X$, obtemos $\left\{U_{1}, \ldots, U_{n}\right\}$.

Seja $\left\{\phi_{1}, \ldots, \phi_{n}\right\}$ uma partição da unidade subordinada à cobertura $\left\{U_{1}, \ldots, U_{n}\right\}$. Para cada $j=1, \ldots, n$, escolha $x_{j} \in U_{j}$ e então $z_{j} \in B_{\mathbb{R}_{\infty}^{N}}\left(f\left(x_{j}\right), \varepsilon / 2\right)$ de modo que $\left\{z_{1}, \ldots, z_{n}\right\}$ está em posição geral em $\mathbb{R}_{\infty}^{N}$.

Mostremos que

$$
\begin{aligned}
g: X & \longrightarrow \mathbb{R}_{\infty}^{N} \\
x & \longmapsto g(x)=\sum_{j=1}^{n} \phi_{j}(x) z_{j}
\end{aligned}
$$

está em $\mathcal{U}_{\delta}$ e que $\sup _{x \in X}\|f(x)-g(x)\|_{\infty}<\varepsilon$.

$\underline{\text { Afirmação 2.1 }} \sup _{x \in X}\|f(x)-g(x)\|_{\infty}<\varepsilon$.

Como $\sum_{j=1}^{n} \phi_{j}(x)=1$, segue que

$$
g(x)-f(x)=\sum_{j=1}^{n} \phi_{j}(x)\left(z_{j}-f\left(x_{j}\right)\right)+\sum_{j=1}^{n} \phi_{j}(x)\left(f\left(x_{j}\right)-f(x)\right) .
$$

Por um lado, sabemos que $\left\|z_{j}-f\left(x_{j}\right)\right\|_{\infty}<\varepsilon / 2$, pela escolha de $z_{j}$. Por outro, como $\sum_{j=1}^{n} \phi_{j}(x)=1$, existe $j_{0} \in\{1, \ldots, n\}$ tal que $\phi_{j_{0}}(x) \neq 0$, daí $x \in \operatorname{supp} \phi_{j_{0}} \subset U_{j_{0}}$ e então

$$
\left\|f\left(x_{j_{0}}\right)-f(x)\right\|_{\infty}<\varepsilon / 2
$$

pois $x, x_{j_{0}} \in U_{j_{0}}$ e $\operatorname{diam} f\left(U_{j_{0}}\right)<\varepsilon / 2$.

Por (3.1.1), segue que $\|g(x)-f(x)\|_{\infty}<\varepsilon$, para todo $x \in X$, consequentemente

$$
\sup _{x \in X}\|g(x)-f(x)\|_{\infty}<\varepsilon .
$$

Afirmação 2.2 $g \in \mathcal{U}_{\delta}$.

Basta mostrar que se $x, y \in X$ são tais que $g(x)=g(y)$, então $x, y \in U_{j_{0}}$, para algum 
$j_{0} \in\{1, \ldots, n\}$, de modo que $\mathrm{d}(x, y)<\delta / 2$ e assim

$$
\Delta(g) \leqslant \delta / 2<\delta
$$

Suponha $x, y \in X$ tais que $g(x)=g(y)$, daí

$$
\sum_{j=1}^{n}\left(\phi_{j}(x)-\phi_{j}(y)\right) z_{j}=0 .
$$

Como a cobertura $\left\{U_{1}, \ldots, U_{n}\right\}$ tem ordem $m+1$, segue que no máximo $m+1$ dos números $\phi_{1}(x), \ldots, \phi_{n}(x)$ são não nulos, pois, caso contrário, teríamos

$$
x \in \bigcap_{k=1}^{m+2} \operatorname{supp} \phi_{j_{k}} \subset \bigcap_{k=1}^{m+2} U_{j_{k}}
$$

para alguma escolha de índices $j_{1}, \ldots, j_{m+2}$ em $\{1, \ldots, n\}$; o que viola o fato de $\left\{U_{1}, \ldots, U_{n}\right\}$ ter ordem $m+1$; e analogamente, no máximo $m+1$ dos números $\phi_{1}(y), \ldots, \phi_{n}(y)$ são não nulos. Assim, a soma $\sum\left(\phi_{j}(x)-\phi_{j}(y)\right) z_{j}$ tem no máximo $2 m+2$ parcelas não nulas.

Por um lado,

$$
\sum_{j=1}^{n}\left(\phi_{j}(x)-\phi_{j}(y)\right)=0
$$

e por outro, como $\left\{z_{1}, \ldots, z_{n}\right\}$ está em posição geral em $\mathbb{R}_{\infty}^{N}$, segue que qualquer subconjunto dele tendo no máximo $N+1$ elementos é G.I..

Sugestivamente, se pusermos $N+1=2 m+2$, então $\phi_{j}(x)-\phi_{j}(y)=0$, para todo $j=1, \ldots, n$. Como antes, existe $j_{0} \in\{1, \ldots, n\}$ tal que $x \in \operatorname{supp} \phi_{j_{0}} \subset U_{j_{0}}$, e uma vez que $\phi_{j_{0}}(y)=\phi_{j_{0}}(x)>0$, concluímos que $y \in \operatorname{supp} \phi_{j_{0}} \subset U_{j_{0}}$, i.e., $x, y \in U_{j_{0}}$, como queríamos.

Que $m$-variedades compactas têm dimensão topológica $m$ é um fato. Mostrar isto, porém, envolve tópicos de topologia algébrica, os quais fogem do intento deste texto.

Naturalmente, pode-se perguntar se $N=2 m+1$ é o menor natural $N$ tal que todo espaço topológico metrizável compacto de dimensão topológica $m$ pode ser imerso em $\mathbb{R}^{N}$. A resposta é afirmativa, mas não é fácil de ser verificada, mesmo quando $m=1$.

Além disso, o Teorema da Imersão admite generalizações que não exigem compacidade. Para mais detalhes, consulte Munkres [25].

Quando se trabalha com espaços métricos separáveis, o Teorema da Imersão faz uso 
das propriedades discutidas na Seção 2.1 e no Apêndice A e ganha a seguinte formulação:

Teorema 3.3. (Teorema da Imersão para Espaços Métricos Separáveis, Jan van Mill [21], Theorem 4.4.4)

Sejam $X$ um espaço métrico separável com $\operatorname{dim}_{\mathrm{T}}(X) \leqslant m e$

$$
\mathcal{R}_{m}=\left\{x \in \mathbb{R}^{2 m+1}: x \text { possui no máximo } m \text { coordenadas racionais }\right\}
$$

Então existe uma imersão $f: X \rightarrow \mathcal{R}_{m} \subset \mathbb{R}^{2 m+1}$ de modo que $f(X)$ é um subconjunto pré-compacto de $\mathcal{R}_{m}$.

A abordagem é basicamente a mesma (Jan Van Mill [21]) que a usada para o Teorema da Imersão tradicional, considerando os conceitos de independência geométrica e posição geral em $\mathbb{R}^{m}$, e segue o roteiro:

1) dados uma função $f \in C\left(X, \mathbb{R}^{2 m+1}\right)$, um hiperplano $H \subset \mathbb{R}^{2 m+1}$ de dimensão no máximo $m$ e uma cobertura aberta finta $\mathcal{U}$ de $X$, então existe uma $\mathcal{U}$-aplicação $g \in C\left(X, \mathbb{R}^{2 m+1}\right)$ que aproxima $f$ e tal que $\overline{g(X)} \cap H=\varnothing ;$

2) provar que $\operatorname{dim}_{\mathrm{T}}\left(\mathcal{R}_{m}\right)=m$;

3) construir uma família enumerável de coberturas abertas finitas e, a partir dela, definir convenientes conjuntos $C_{n}$ abertos densos em $C\left(X, \mathbb{R}^{2 m+}\right)$;

4) do Teorema da Categoria de Baire (Teorema 1.14), $C=\bigcap_{n=1}^{\infty} C_{n}$ é não vazio e fornece a imersão desejada $f$.

Neste contexto, provar que $2 m+1$ é a dimensão ótima torna-se menos difícil e o autor Jan Van Mill [21] indica as devidas referências para tal demonstração.

\subsection{Projeções de Compactos e o Teorema de Mañé}

Como já mencionamos, gostaríamos de enxergar os atratores de semigrupos como objetos de dimensão finita, mesmo quando o semigrupo está num espaço de Banach de dimensão infinita. Para isso, vamos mostrar que conjuntos compactos que têm dimensão fractal finita podem ser projetados, de maneira injetiva, em um espaço vetorial de dimensão finita. Este resultado é encontrado em Carvalho [8] e Mañé [24], e a ele nos referimos 
como o Teorema de Mañé (Teorema 3.6). É sobre ele que recai a responsabilidade de lincar a Teoria das Dimensões à Teoria dos Semigrupos, e por conta disso podemos tomá-lo como o resultado chave deste texto.

O tratamento por vir é feito sobre espaços de Banach, uma consideração bastante usual em problemas de equações diferenciais e, neste caso, necessária uma vez que precisamos da estrutura de espaço normado.

Encerramos a seção com o corolário do Teorema de Mañé, que usa a dimensão fractal em vez de a Hausdorff. Isto porque queremos evitar trabalhar com o conjunto $K-K$, que pode ser surpreendentemente complicado; com a dimensão fractal, basta considerar o conjunto $K$ por si só. Nestas condições, obtemos o resultado que efetivamente aplica a Teoria das Dimensões à Teoria de Semigrupos.

Se $X$ é um espaço de Banach e $Y$ é um subespaço vetorial fechado de $X$, seja

$$
\mathcal{P}(X, Y):=\left\{P \in \mathcal{L}(X): P^{2}=P \text { e } P(X)=Y\right\}
$$

com a topologia uniforme de operadores, ou seja, a topologia induzida pela norma

$$
\|T\|_{\mathcal{L}(X)}=\sup _{\substack{x \in X \\\|x\|_{X}=1}}\|T x\|_{X}
$$

Nestas condições, $\mathcal{P}(X, Y)$ é um subespaço fechado de $\mathcal{L}(X)$, logo um espaço de Banach. Isto nos permite aplicar o Teorema da Categoria de Baire (Teorema 1.14), se for necessário.

Observação 3.4. Se $Y$ tem dimensão finita, então $\mathcal{P}(X, Y) \neq \varnothing$.

Seja $m=\operatorname{dim} Y$ finito.

Tome $\left\{e_{1}, \ldots, e_{m}\right\}$ uma base de $Y$ e uma correspondente $\left\{e_{1}^{*}, \ldots, e_{m}^{*}\right\}$ base dual:

$$
<e_{i}^{*}, e_{j}>=\delta_{i, j}
$$

Pelo Teorema de Hahn-Banach (Teorema 1.17), seja $P_{i}: X \rightarrow \mathbb{C}$ uma extensão contínua de $e_{i}^{*}$ a todo $X$; defina

$$
\begin{aligned}
P: X & \longrightarrow \\
x & \longmapsto \sum_{i=1}^{m}<P_{i}, x>e_{i},
\end{aligned}
$$


daí $R(P) \subset Y$ e $P x=x$, para todo $x \in Y$; com isso, $R(P)=Y$. É fácil verificar também que $P^{2}=P, \log$ $P \in \mathcal{P}(X, Y)$, de modo que $\mathcal{P}(X, Y) \neq \varnothing$.

Esta observação é essencial pois nos diz que existem projeções de $X$ em $Y$, quando $Y$ tem dimensão finita, de modo que a busca por uma que seja injetora "em algum sentido" não é em vão: há candidatos!

O próximo lema fixa notações e fornece resultados invocados na demonstração do Teorema 3.6 de Mañé.

Lema 3.5. Sejam $X$ um espaço de Banach, $Y$ um subespaço vetorial de $X$ com dimensão finita e $K$ reunião enumerável de compactos $K_{n} \subset X$.

Então, valem as seguintes afirmações:

a) existem funcionais $\phi_{1}, \phi_{2}, \ldots$ em $X^{*}$ tais que se $x \in \operatorname{span}(K)$ e $\phi_{n}(x)=0$, para todo $n$ natural, então $x=0$;

b) o conjunto

$$
A_{n, r}=\left(K_{n}-K_{n}\right) \cap\left\{x \in X:\|x\|_{X} \geqslant r\right\}
$$

é um compacto de $X$, para todo $r>0$ e todo $n$ natural;

c) dado $P \in \mathcal{P}(X, Y)$, tem-se que $\operatorname{ker}(P) \cap A_{n, r}=\varnothing$ se, e somente se, $P \in \mathcal{P}_{n, r}$, com

$$
\mathcal{P}_{n, r}=\left\{P \in \mathcal{P}(X, Y): \operatorname{diam}\left(P^{-1}(\{y\}) \cap K_{n}\right)<r, \forall y \in Y\right\}
$$

d) $\mathcal{P}_{n, r}$ é um aberto de $\mathcal{P}(X, Y)$.

\section{Demonstração:}

Item a): Seja $W$ o fecho do subespaço vetorial de $X$ gerado por $K$. Como $K$ é separável (por ser $\sigma$-compacto), segue que $W$ é um espaço de Banach separável.

Uma vez que $B_{W^{*}}[0,1]$ é compacta e metrizável na topologia fraca* $\sigma\left(W^{*}, W\right)$, graças aos Teoremas 1.18 e 1.19, segue que existe $\left\{\phi_{1}, \phi_{2}, \ldots\right\}$ subconjunto enumerável denso em $\left(B_{W^{*}}[0,1], \sigma\left(W^{*}, W\right)\right)$.

Seja $x \in W$ tal que $\phi_{n}(x)=0$, para todo $n$.

Toda $\phi \in B_{W^{*}}[0,1]$ é $\sigma\left(W^{*}, W\right)$-limite de alguma subsequência de $\left(\phi_{n}\right)_{n}$, de modo que $\phi(x)=0$ e então $x=0$, pelos Lema 1.16 e Teorema 1.17. 
Agora, basta estender tais funcionais $\left(\phi_{n}\right)_{n} \subset W^{*}$ a $X^{*}$ pelo Teorema de Hahn-Banach (Teorema 1.17). Note que podemos tomá-los de modo que $\left\|\phi_{n}\right\|_{X^{*}}=1$.

Item b): Uma vez que a aplicação

$$
X \times X \ni(x, y) \longmapsto x-y \in X
$$

é contínua, segue que $K_{n}-K_{n}$ é um compacto de $X$, logo também $A_{n, r}$ é compacto.

Item c): Mostremos a ida: suponha $\operatorname{ker}(P) \cap A_{n, r}=\varnothing$.

Dado $y \in Y$, sejam $v, w \in P^{-1}(\{y\}) \cap K_{n}$, daí $v-w \in \operatorname{ker}(P)$ e $\|v-w\|_{X}<r$.

A compacidade de $P^{-1}(\{y\}) \cap K_{n}$ impede que o supremo de $\|v-w\|_{X}$ sobre tais $v$ e $w$ atinja o valor $r$, isto é, $\operatorname{diam}\left(P^{-1}(\{y\}) \cap K_{n}\right)<r$.

Quanto à contrapositiva da volta, suponha $y \in \operatorname{ker}(P) \cap A_{n, r}$, então $P y=0$ e existem $v, w \in K_{n}$ tais que $y=v-w$ e $\|v-w\|_{X} \geqslant r$. Pondo $z:=P v=P w \in Y$, segue que $\operatorname{diam}\left(P^{-1}(\{z\}) \cap K_{n}\right) \geqslant\|v-w\|_{X} \geqslant r, \log \mathrm{P} \notin \mathcal{P}_{n, r}$.

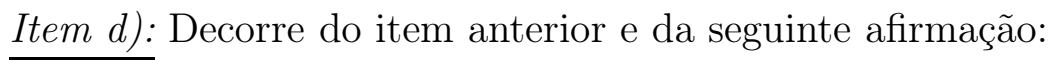

Afirmação: Se $J \subset X$ é um compacto, então o conjunto

$$
\{P \in \mathcal{P}(X, Y): \operatorname{ker}(P) \cap J=\varnothing\}
$$

é um aberto de $\mathcal{P}(X, Y)$.

De fato, sejam $P_{0} \in \mathcal{P}(X, Y)$ tal que $\operatorname{ker}\left(P_{0}\right) \cap J=\varnothing$ e $\varepsilon_{0}=\mathrm{d}\left(\operatorname{ker}\left(P_{0}\right), J\right)$, de modo que $\varepsilon_{0}>0$. Fixemos $s, t \in \mathbb{R}$ tais que $J \subset B_{X}(0, t)$ e $s>2 \varepsilon_{0}+t$.

Se $P \in \mathcal{P}(X, Y)$ é tal que $\left\|P_{0}-P\right\|_{\mathcal{L}(X, Y)}<\varepsilon_{0} / s$, então

$$
\begin{aligned}
\mathrm{d}(\operatorname{ker}(P), J) & =\mathrm{d}\left(\left(I_{X}-P\right) X, J\right) \\
& \stackrel{(1)}{=} \mathrm{d}\left(\left(I_{X}-P\right) B_{X}(0, s), J\right) \\
& \stackrel{(2)}{\geqslant} \mathrm{d}\left(\left(I_{X}-P_{0}\right) B_{X}(0, s), J\right)-\sup _{x \in B_{X}(0, s)}\left\|P x-P_{0} x\right\|_{X} \\
& =\mathrm{d}\left(\operatorname{ker}\left(P_{0}\right), J\right)-s\left\|P-P_{0}\right\|_{\mathcal{L}(X)}>0,
\end{aligned}
$$

consequentemente $\operatorname{ker}(P) \cap J=\varnothing$. 
Justifiquemos as passagens (1) e (2). Quanto à (2), fixados $x \in B_{X}(0, s)$ e $u \in J$, temos que

$$
\left\|\left(I_{X}-P_{0}\right) x-u\right\|_{X} \leqslant\left\|\left(I_{X}-P\right) x-u\right\|_{X}+\left\|\left(P-P_{0}\right) x\right\|_{X}
$$

e então

$$
\left\|\left(I_{X}-P_{0}\right) x-u\right\|_{X} \leqslant\left\|\left(I_{X}-P\right) x-u\right\|_{X}+\sup _{x \in B_{X}(0, s)}\left\|\left(P-P_{0}\right) x\right\|_{X}
$$

de modo que passando o ínfimo sobre todos os $x \in B_{X}(0, s)$ e $u \in J$, obtemos

$$
\mathrm{d}\left(\left(I_{X}-P_{0}\right) B_{X}(0, s), J\right) \leqslant \mathrm{d}\left(\left(I_{X}-P\right) B_{X}(0, s), J\right)+\sup _{x \in B_{X}(0, s)}\left\|\left(P-P_{0}\right) x\right\|_{X}
$$

como queríamos.

Agora, para (1), claro que $\mathrm{d}\left(\left(I_{X}-P\right) X, J\right) \leqslant \mathrm{d}\left(\left(I_{X}-P\right) B_{X}(0, s), J\right)$. Resta verificar que vale a igualdade. Se não fosse assim, encontraríamos $\delta>0$ tal que d $\left(\left(I_{X}-P\right) X, J\right)+$ $\delta<\mathrm{d}\left(\left(I_{X}-P\right) B_{X}(0, s), J\right)$.

Como d $(\operatorname{ker}(P), J)$ é o ínfimo de $\left\|\left(I_{X}-P\right) x-u\right\|_{X}$ sobre todos os $x \in X$ e $u \in J$, tome, para cada $n$, pontos $x_{n} \in X$ e $u_{n} \in J$ tais que

$$
\left\|\left(I_{X}-P\right) x_{n}-u_{n}\right\|_{X}<\mathrm{d}(\operatorname{ker}(P), J)+1 / n
$$

Graças à compacidade de $J \subset B_{X}(0, t)$, temos $\left\|u_{n}\right\|_{X}+1 / n<t$, para todo $n$ suficientemente grande, e daí

$$
\left\|\left(I_{X}-P\right) x_{n}\right\|<t+\mathrm{d}(\operatorname{ker}(P), J) .
$$

Em particular, quando $P=P_{0}$, temos $\left\|\left(I_{X}-P_{0}\right) x_{n}\right\|<t+\varepsilon_{0}<s$ e isto nos conduz ao absurdo de $0<\delta<1 / n$, para todo $n$ suficientemente grande:

$$
\begin{aligned}
\mathrm{d}\left(\operatorname{ker}\left(P_{0}\right), J\right)+\delta & <\left\|\left(I_{X}-P_{0}\right)(\underbrace{\left(I_{X}-P_{0}\right) x_{n}}_{\in B_{X}(0, s)})-u_{n}\right\|_{X} \\
& =\left\|\left(I_{X}-P_{0}\right) x_{n}-u_{n}\right\|_{X} \\
& <\mathrm{d}\left(\operatorname{ker}\left(P_{0}\right), J\right)+1 / n .
\end{aligned}
$$

Ou seja, a igualdade $\mathrm{d}\left(\left(I_{X}-P_{0}\right) X, J\right)=\mathrm{d}\left(\left(I_{X}-P_{0}\right) B_{X}(0, s), J\right)$ de fato ocorre. E note que, para obter o absurdo, foi suficiente que $\left(I_{X}-P_{0}\right) x_{n}$ estivesse na bola $B_{X}(0, s)$ 
para todo $n$ suficientemente grande. O mesmo ocorre para $P$ :

$$
\begin{aligned}
\mathrm{d}(\operatorname{ker}(P), J) & =\inf _{\substack{x \in X \\
u \in J}}\left(\left\|\left(I_{X}-P\right) x-u\right\|_{X}\right) \\
& \leqslant \inf _{\substack{x \in B_{X}(0, s) \\
u \in J}}\left(\left\|\left(I_{X}-P\right) x-u\right\|_{X}\right) \\
& \leqslant \inf _{\substack{x \in B_{X}(0, s) \\
u \in J}}\left(\left\|\left(I_{X}-P_{0}\right) x-u\right\|_{X}\right)+\sup _{\substack{x \in B_{X}(0, s) \\
u \in J}}\left\|\left(P_{0}-P\right) x\right\|_{X} \\
& =\varepsilon_{0}+s\left\|P_{0}-P\right\|_{\mathcal{L}(X, Y)} \\
& <2 \varepsilon_{0},
\end{aligned}
$$

e usando que $s>2 \varepsilon_{0}+t$, temos $\left\|\left(I_{X}-P\right) x_{n}\right\|_{X}<s$, o que nos conduz ao mesmo absurdo de antes.

Portanto, vale a igualdade

$$
\mathrm{d}\left(\left(I_{X}-P\right) X, J\right)=\mathrm{d}\left(\left(I_{X}-P\right) B_{X}(0, s), J\right) .
$$

Vamos mostrar que, dados um $\sigma$-compacto $K$ de um espaço de Banach $X$ e $Y$ um subespaço de dimensão finita, existe uma projeção de $P \in \mathcal{P}(X, Y)$ de tal forma que $\left.P\right|_{K}$ é injetora. Para tanto, o Teorema da Categoria de Baire é invocado mais uma vez e, claro, condições são impostas sobre a dimensão de Hausdorff de $K$ como prometido; mais precisamente, sobre a dimensão de Hausdorff de $K-K$, um conjunto que pode ser bem "bagunçado", mesmo que $K$ seja um compacto "simples".

Precisamos de um roteiro a ser seguido para facilitar o entendimento da demonstração e reconhecer o paralelo com o Teorema da Imersão (Teorema 3.2). Denote por $\mathcal{P}_{\text {inj a }}$ família das projeções $P \in \mathcal{P}(X, Y)$ com a propriedade de que $\left.P\right|_{K}$ é injetora.

1) $\mathcal{P}_{\text {inj }}$ ser intersecção enumerável dos abertos $\mathcal{P}_{n, 1 / m}$ ainda não é suficiente;

2) construir conjuntos $\mathcal{P}_{n, r, i, j} \subset \mathcal{P}(X, Y)$ abertos tais que

$$
\mathcal{P}_{n, r}=\bigcap_{i \in \mathbb{N}} \bigcap_{j \in \mathbb{N}} \mathcal{P}_{n, r, i, j}
$$


3) dados $P_{0} \in \mathcal{P}(X, Y)$ e $\varepsilon>0$, construir uma projeção $P_{\varepsilon} \in \mathcal{P}_{n, r, i, j}$ tal que

$$
\left\|P_{\varepsilon}-P_{0}\right\|_{\mathcal{P}(X, Y)} \leqslant \varepsilon
$$

4) do Teorema da Categoria de Baire,

$$
\mathcal{P}_{\text {inj }}=\bigcap_{n, m, i, j \in \mathbb{N}} \mathcal{P}_{n, 1 / m, i, j}
$$

é denso em $\mathcal{P}(X, Y)$, de onde segue que $K$ pode ser projetado injetivamente em $Y$.

\section{Teorema 3.6. (Teorema de Mañé, [24])}

Sejam $X$ um espaço de Banach sobre $\mathbb{R}, Y$ um subespaço vetorial de $X$ e $K$ reunião enumerável crescente de compactos $K_{n} \subset X$ tais que

$$
\operatorname{dim}_{\mathrm{H}}(K-K)+1<\operatorname{dim}(Y)<\infty \text {. }
$$

Então existe uma projeção $P \in \mathcal{P}(X, Y)$ tal que $\left.P\right|_{K}$ é injetora.

\section{Demonstração:}

Usando a definição de $\mathcal{P}_{n, r}$, não é difícil verificar que

$$
\mathcal{P}_{\text {inj }}=\bigcap_{n \in \mathbb{N}} \bigcap_{m \in \mathbb{N}} \mathcal{P}_{n, 1 / m}
$$

De fato, $\left.P\right|_{K}$ é injetora se, e somente se, $\left.P\right|_{K_{n}}$ é injetora, para cada $n$, se, e somente se, $\operatorname{diam}\left(P^{-1}(\{y\}) \cap K_{n}\right)=0$, para cada $n$ natural e $y \in Y$, se, e somente se, $\operatorname{diam}\left(P^{-1}(\{y\}) \cap K_{n}\right)<1 / m$, para todos os $n, m \in \mathbb{N}$ e $y \in Y$, se, e somente se, $P \in \mathcal{P}_{n, 1 / m}$ para todos os $n, m \in \mathbb{N}$.

Seja $Q$ a aplicação quociente de $X$ sobre $Z:=X / Y$, daí $Q\left(A_{n, r}\right) \backslash\{0\}$ é um conjunto $\sigma$-compacto de $Z$ :

$$
Q\left(A_{n, r}\right) \backslash\{0\}=\bigcup_{m \in \mathbb{N}}\left\{Q(x): x \in A_{n, r},\|Q(x)\|_{Z} \geqslant 1 / m\right\}
$$

em que cada $\left\{Q(x): x \in A_{n, r},\|Q(x)\|_{Z} \geqslant 1 / m\right\}$ é compacto em $Z$, pois $Q$ é um operador contínuo com $\|Q\|_{\mathcal{L}(X, Z)} \leqslant 1$. 
Dado $x \in A_{n, r}$, claro que $Q(x) \in \operatorname{span}\left(Q\left(A_{n, r}\right) \backslash\{0\}\right)$. Pelo Lema 3.5, existem funcionais $\phi_{1}, \phi_{2}, \ldots$ em $Z^{*}$ tais que se $x \in A_{n, r}$ é tal que $\phi_{i}(Q(x))=0$, para todo $i$ natural, então $Q(x)=0$, ou seja, $x \in Y$. Com isso, podemos escrever

$$
A_{n, r}=\left(\bigcup_{i \in \mathbb{N}} \bigcup_{j \in \mathbb{N}}\left\{x \in A_{n, r}:\left|\phi_{i}(Q(x))\right| \geqslant 1 / j\right\}\right) \cup\left(A_{n, r} \cap Y\right)
$$

e, para cada $P \in \mathcal{P}(X, Y)$, como $A_{n, r} \not \ngtr 0$, segue que

$$
\operatorname{ker}(P) \cap A_{n, r}=\bigcup_{i \in \mathbb{N}} \bigcup_{j \in \mathbb{N}}\left(\operatorname{ker}(P) \cap\left\{x \in A_{n, r}:\left|\phi_{i}(Q(x))\right| \geqslant 1 / j\right\}\right)
$$

Sejam $A_{n, r, i, j}:=\left\{x \in A_{n, r}:\left|\phi_{i}(Q(x))\right| \geqslant 1 / j\right\}$, o qual é compacto, pois é subconjunto fechado de $A_{n, r} \subset X$, e

$$
\mathcal{P}_{n, r, i, j}:=\left\{P \in \mathcal{P}(X, Y): \operatorname{ker}(P) \cap A_{n, r, i, j}=\varnothing\right\}
$$

o qual é aberto em $\mathcal{P}(X, Y)$ (basta proceder como no item d) do Lema 3.5) e satisfaz

$$
\mathcal{P}_{n, r}=\bigcap_{i \in \mathbb{N}} \bigcap_{j \in \mathbb{N}} \mathcal{P}_{n, r, i, j}
$$

pois $P \in \mathcal{P}_{n, r}$ se, e somente se, $\operatorname{ker}(P) \cap A_{n, r}=\varnothing$ se, e somente se, $\operatorname{ker}(P) \cap A_{n, r, i, j}=\varnothing$, para todos os naturais $i$ e $j$. Agora, por (3.2.1),

$$
\mathcal{P}_{\text {inj }}=\bigcap_{n, m, i, j \in \mathbb{N}} \mathcal{P}_{n, 1 / m, i, j}
$$

é intersecção enumerável de abertos.

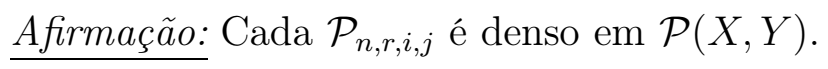

Sejam $P_{0} \in \mathcal{P}(X, Y)$ e $\varepsilon>0$; defina

$$
\begin{aligned}
\Psi: Y \backslash\{0\} & \longrightarrow S_{Y}(0,1) \\
y & \longmapsto \frac{y}{\|y\|_{X}}
\end{aligned}
$$

Primeiro, note que $\Psi$ restrito a $P_{0}\left(A_{n, r}\right) \cap\left(Y \backslash B_{Y}(0,1 / k)\right)$ é $(2 k)$-Lipschitz contínua, 
para cada $k$ natural; daí pelo Teorema 2.28 vale

$$
\operatorname{dim}_{\mathrm{H}}\left(\Psi\left(P_{0}\left(A_{n, r}\right) \cap\left(Y \backslash B_{Y}(0,1 / k)\right)\right)\right) \leqslant \operatorname{dim}_{\mathrm{H}}\left(P_{0}\left(A_{n, r}\right)\right)
$$

e como $P_{0}\left(A_{n, r}\right) \backslash\{0\}$ é reunião enumerável dos conjuntos $P_{0}\left(A_{n, r}\right) \cap\left(Y \backslash B_{Y}(0,1 / k)\right)$, segue do Teorema 2.24, do Teorema 2.28 e da monotonicidade da dimensão de Hausdorff que

$$
\begin{aligned}
\operatorname{dim}_{\mathrm{H}}\left(\Psi\left(P_{0}\left(A_{n, r}\right) \backslash\{0\}\right)\right) & =\sup _{k \in \mathbb{N}} \operatorname{dim}_{\mathrm{H}}\left(\Psi\left(P_{0}\left(A_{n, r}\right) \cap\left(Y \backslash B_{Y}(0,1 / k)\right)\right)\right) \\
& \leqslant \operatorname{dim}_{\mathrm{H}}\left(P_{0}\left(A_{n, r}\right)\right) \\
& \leqslant \operatorname{dim}_{\mathrm{H}}\left(A_{n, r}\right) \\
& \leqslant \operatorname{dim}_{\mathrm{H}}\left(K_{n}-K_{n}\right) \\
& \leqslant \operatorname{dim}_{\mathrm{H}}(K-K) \\
& <\operatorname{dim}(Y)-1 .
\end{aligned}
$$

Sendo assim, deve existir $y_{0} \in S_{Y}(0,1) \backslash\left(\Psi\left(P_{0}\left(A_{n, r}\right) \backslash\{0\}\right)\right)$; pois caso contrário, teríamos a igualdade $S_{Y}(0,1)=\Psi\left(P_{0}\left(A_{n, r}\right) \backslash\{0\}\right)$ e, uma vez que $S_{Y}(0,1)$ é uma hiperfície de $Y$, chegamos ao absurdo de

$$
\operatorname{dim}(Y)-1=\operatorname{dim}_{\mathrm{H}}\left(S_{Y}(0,1)\right)=\operatorname{dim}_{\mathrm{H}}\left(\Psi\left(P_{0}\left(A_{n, r}\right) \backslash\{0\}\right)\right)<\operatorname{dim}(Y)-1
$$

Agora podemos definir, para cada $x \in X$,

$$
P_{\varepsilon}(x):=P_{0}(x)+\varepsilon \phi_{i}(Q(x)) y_{0}
$$

de modo que $P_{\varepsilon} \in \mathcal{P}(X, Y)$ e $\left\|P_{0}-P_{\varepsilon}\right\|_{\mathcal{L}(X)} \leqslant \varepsilon\left\|\phi_{i}\right\|_{Z^{*}}\left\|y_{0}\right\|_{X}\|Q\|_{\mathcal{L}(X, Z)} \leqslant \varepsilon$.

Basta enfim verificar que $P_{\varepsilon} \in \mathcal{P}_{n, r, i, j}$, i.e., que $\operatorname{ker}\left(P_{\varepsilon}\right) \cap A_{n, r, i, j}=\varnothing$.

De fato, se existisse $x \in \operatorname{ker}\left(P_{\varepsilon}\right) \cap A_{n, r, i, j}$, então $P_{0}(x)=-\varepsilon \phi_{i}(Q(x)) y_{0} \neq 0$, pois $\phi_{i}(Q(x)) \geqslant 1 / j$; em outras palavras, $P_{0}(x) \in P_{0}\left(A_{n, r}\right) \backslash\{0\}$.

Como $y_{0}=\Psi\left(y_{0}\right)$, segue que

$$
\pm y_{0}= \pm \Psi\left(y_{0}\right)=\frac{-\varepsilon \phi_{i}(Q(x)) y_{0}}{\left\|-\varepsilon \phi_{i}(Q(x)) y_{0}\right\|_{X}}=\Psi\left(P_{0}(x)\right) \in \Psi\left(P_{0}\left(A_{n, r}\right) \backslash\{0\}\right)
$$


o que contraria a escolha de $y_{0}$.

Portanto, $P_{\varepsilon} \in \mathcal{P}_{n, r, i, j}$.

Finalmente, como

$$
\mathcal{P}_{\text {inj }}=\bigcap_{n, m, i, j \in \mathbb{N}} \mathcal{P}_{n, 1 / m, i, j}
$$

é intersecção enumerável de abertos densos em $\mathcal{P}(X, Y)$, segue do Teorema da Categoria de Baire que $\mathcal{P}_{\text {inj }}$ é denso em $\mathcal{P}(X, Y)$; em particular, existe uma projeção $P: X \rightarrow Y$ que, quando restrita a $K$, é uma aplicação injetora.

Vale observar que todo conjunto $\sigma$-compacto $K \subset X$ pode ser escrito como reunião enumerável crescente de compactos de $X$, de modo que o enunciado do Teorema 3.6 pode ser simplificado ao pedir que $K$ seja $\sigma$-compacto.

Finalmente, o resultado que norteou nossos esforços segue do Teorema 3.6 como corolário.

Corolário 3.7. Seja $\{T(t): t \geqslant 0\}$ um semigrupo em um espaço de Banach $X$ com atrator global $\mathcal{A}$.

Se $\operatorname{dim}_{\mathrm{F}}(\mathcal{A})<\infty$, então $\mathcal{A}$ pode ser projetado injetivamente em $\mathbb{R}^{N}$, para $N$ suficientemente grande.

\section{Demonstração:}

Sabidamente, $\mathcal{A}$ é compacto e a dimensão fractal domina a de Hausdorff.

Tome $N$ natural grande o suficiente para que

$$
\operatorname{dim}_{\mathrm{H}}(\mathcal{A}-\mathcal{A})+1 \leqslant 2 \operatorname{dim}_{\mathrm{F}}(\mathcal{A})+1<N
$$

e seja $Y$ um subespaço de $X$ com dimensão $N$.

Pelo Teorema 3.6, $\mathcal{A}$ pode ser projetado injetivamente em $Y$, o qual é isometricamente isormofo a $\mathbb{R}^{N}$, e isto encerra a demonstração. 


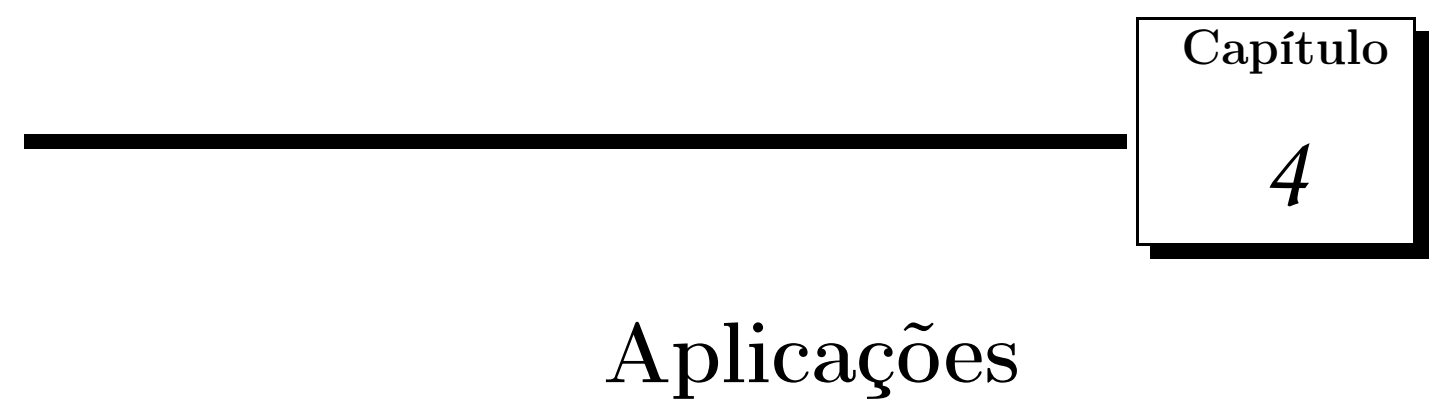

Agora que entendemos de que maneira estudar dimensões é útil para investigar a dinâmica de um atrator, vamos explorar métodos para estimar a dimensão fractal de atratores de semigrupos.

Os semigrupos gradientes reaparecem, muito por conta de suas nobres propriedades dinâmicas. Estudamos também a finitude da dimensão fractal de atratores exponenciais, o que também promove $\operatorname{dim}_{\mathrm{F}}(\mathcal{A})<\infty$. Por fim, o estudo de compactos invariante estabelece critérios para que o atrator de um semigrupo $\{T(t): t \geqslant 0\}$ tenha dimensão finita.

Podemos dizer que os semigrupos gradientes são uma classe seleta de semigrupos. Mas, de modo geral, os atratores têm uma taxa de atração exponencial e este caso é contemplado quando estudamos os atratores exponenciais. Quanto aos que não têm tal taxa, aplicamos a teoria da Seção 4.3. Em todos os três casos, o Corolário 3.7 é invocado, de modo que os respectivos atratores podem ser projetados injetivamente em espaços finito dimensionais.

\subsection{Atratores de Semigrupos Gradientes}

Agora, devemos obter estimativas da dimensão fractal do atrator global dos semigrupos gradientes $(T(\cdot), \Xi)$ em termos da dimensão fractal dos conjuntos instáveis locais dos invariantes isolados de $\Xi$; das propriedades de atração exponencial do semigrupo e das propriedades Lipschitz do semigrupo. Vamos apresentar os resultados pedindo que $X$ seja espaço métrico e só então quando formos aplicar o Teorema [3.7, será exigido que $X$ seja 
um espaço de Banach. As principais referências são [3] e Carvalho [8].

Observação 4.1. Sejam $(T(\cdot), \Xi)$ um semigrupo gradiente com atrator $\mathcal{A}$ e $\left\{S^{n}: n \in \mathbb{Z}_{+}\right\}$ o semigrupo gradiente discreto obtido pondo $S=T(1)$, com atrator $\mathcal{A}^{\prime}$. Acontece que $\mathcal{A}$ e $\mathcal{A}^{\prime}$ coincidem.

De fato, como

$$
\mathcal{A}=\{x \in X: \text { existe uma solução global limitada } \xi: \mathbb{R} \rightarrow X \text { tal que } \xi(0)=x\}
$$

e

$\mathcal{A}^{\prime}=\{x \in X:$ existe uma solução global limitada $\phi: \mathbb{Z} \rightarrow X$ tal que $\phi(0)=x\}$

já obtemos que $\mathcal{A} \subset \mathcal{A}^{\prime}$.

Reciprocamente, dado $z \in \mathcal{A}^{\prime}$, existem uma solução global discreta limitada $\phi: \mathbb{Z} \rightarrow X$ tal que $\phi(0)=z$, e invariantes isolados $E_{i}$ e $E_{j}$ tais que $E_{i} \stackrel{n \rightarrow-\infty}{\longleftarrow} \phi(n) \stackrel{n \rightarrow \infty}{\longrightarrow} E_{j}$.

Defina $\xi: \mathbb{R} \rightarrow X$ por $\xi(t)=T(t) \phi(0)$, para todo $t \geqslant 0$, e

$$
\xi(-t)=T(n-t) \phi(-n)
$$

para todo $t \in[0, n]$.

Note que $\xi$ está bem definida e é contínua. Se $t \in[0, n]$ e também $t \in[0, n+k]$, mostremos que $\xi(-t)$ independe do intervalo escolhido. De fato, pondo $m=n+k$, temos

$$
\begin{aligned}
\xi(-t) & =T(m-t) \phi(-m) \\
& =T(k) T(n-t)(T(-k) \phi(-n)) \\
& =(T(k) T(-k)) T(n-t) \phi(-n) \\
& =T(n-t) \phi(-n) .
\end{aligned}
$$

Não é difícil conferir que $\xi$ é uma solução global para $T(\cdot)$ e que $\left.\xi\right|_{\mathbb{Z}} \equiv \phi$.

Resta verificar que $\{\xi(t): t \in \mathbb{R}\}$ é limitado em $X$. Sabemos que para todo $n$ natural grande o suficiente, temos $\phi(n) \in \mathcal{O}_{1}\left(E_{j}\right)$. Se $\{\xi(t): t \geqslant 0\}$ não fosse limitado, existiriam 
$t_{k} \rightarrow \infty$ e $n_{k} \in \mathbb{N}$ tais que $t_{k}-n_{k} \in[0,1] \mathrm{e}$

$$
\mathrm{d}\left(\xi\left(t_{k}\right), \mathcal{O}_{1}\left(E_{j}\right)\right) \geqslant k
$$

Uma vez que $\left(\phi\left(n_{k}\right)\right)_{k}$ é uma sequência em $E_{j} \subset \mathcal{A}^{\prime}$ e $\left(t_{k}-n_{k}\right)_{k}$ é uma sequência em $[0,1]$, podemos assumir que $\phi\left(n_{k}\right) \rightarrow x_{0} \in E_{j}$ e que $t_{k}-n_{k} \rightarrow t_{0} \in[0,1]$, de modo que

$$
\mathrm{d}\left(\xi\left(t_{k}\right), E_{j}\right)=\mathrm{d}\left(T\left(t_{k}-n_{k}\right) \xi\left(n_{k}\right), E_{j}\right) \rightarrow \mathrm{d}\left(T\left(t_{0}\right) x_{0}, E_{j}\right)=0,
$$

pois $E_{j}$ é invariante. Mas isto contraria (4.1.1).

Analogamente, verifica-se que $\{\xi(t): t \leqslant 0\}$ é limitado.

Note que as propriedades gradientes do semigrupo não foram necessárias para obter $\mathcal{A} \subset \mathcal{A}^{\prime}$, de modo que a inclusão permanece válida para qualquer semigrupo.

A Observação 4.1 nos diz que, a fim de estimar $\operatorname{dim}_{F}(\mathcal{A})$, podemos trabalhar com atratores de semigrupos gradientes discretos.

Para o estudo dos semigrupos gradientes (e do tipo gradiente), o conceito de par atrator-repulsor é grande ajuda, pois além de construir a decomposição de Morse, a qual é essencial para estabelecer a equivalência entre as duas classes de semigrupos, vai nos permitir limitar a dimensão fractal do atrator global.

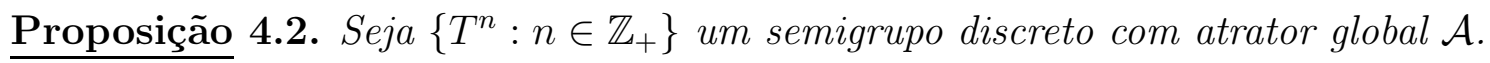

Dado um par atrator-repulsor $\left(A, A^{*}\right)$, suponha que

i) exista uma vizinhança $B$ de $A^{*}$ em $\mathcal{A}$ tal que $S=\left.T\right|_{B}$ é uma aplicação c-Lipschitz, $\operatorname{com} c>1$;

ii) existam constantes $M \geqslant 1$ e $\omega>0$ tais que

$$
\mathrm{d}_{\mathrm{H}}\left(S^{n}(K), A\right) \leqslant M e^{-\omega n}, \forall n \in \mathbb{N},
$$

para todo $K \subset \mathcal{A}$ compacto disjunto de $A^{*}$.

Então

$$
\operatorname{dim}_{\mathrm{F}}(B) \leqslant \operatorname{dim}_{\mathrm{F}}(\mathcal{A}) \leqslant \max \left\{\frac{\omega+\log c}{\omega} \operatorname{dim}_{\mathrm{F}}(B) ; \operatorname{dim}_{\mathrm{F}}(A)\right\}
$$


Demonstração: Como $B \subset \mathcal{A}$ é totalmente limitado, faz sentido calcular sua dimensão fractal e claro que $\operatorname{dim}_{\mathrm{F}}(B) \leqslant \operatorname{dim}_{\mathrm{F}}(\mathcal{A})$.

A fim de provar a segunda desigualdade, dividimos a prova em dois passos:

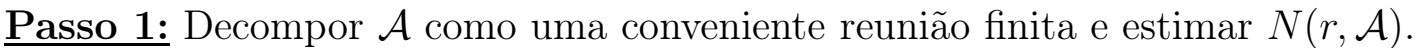

Em primeiro lugar, verifiquemos que $N\left(r, \Omega_{n}\right) \leqslant N\left(r / c^{n}, \Omega_{0}\right)$, em que

$$
\Omega_{n}=S^{n}(\mathcal{A} \backslash B) \backslash S^{n+1}(\mathcal{A} \backslash B),
$$

para cada $n=0,1,2, \ldots$

Fixado $n$, seja $N_{0}=N\left(r / c^{n}, \Omega_{0}\right)$, tome pontos $x_{1}, \ldots, x_{N_{0}}$ em $\Omega_{0}$ tais que

$$
\Omega_{0} \subset \bigcup_{j=1}^{N_{0}} B_{X}\left(x_{j}, r / c^{n}\right)
$$

e considere os pontos $z_{j}=S^{n} x_{j}$, de modo que $z_{j} \in \Omega_{n}$, para cada $j=1, \ldots, N_{0}$, pela definição de $\Omega_{n}$.

Como, alternativamente, podemos escrever

$$
\Omega_{n}=S^{n}((\mathcal{A} \backslash B) \backslash S(\mathcal{A} \backslash B))=S^{n}\left(\Omega_{0}\right),
$$

para cada $z \in \Omega_{n}$, existe $x \in \Omega_{0}$ tal que $z=S^{n} x$ e $x \in B\left(x_{j}, r / c^{n}\right)$, para algum $j=1, \ldots, N_{0}$. Deste modo,

$$
\mathrm{d}\left(z, z_{j}\right)=\mathrm{d}\left(S^{n} x, S^{n} x_{j}\right) \leqslant c^{n} \mathrm{~d}\left(x, x_{j}\right)<r
$$

e então

$$
\Omega_{n} \subset \bigcup_{j=1}^{N_{0}} B_{X}\left(z_{j}, r\right),
$$

de onde obtemos que $N\left(r, \Omega_{n}\right) \leqslant N_{0}=N\left(r / c^{n}, \Omega_{0}\right)$.

Fixado $r>0$, uma vez que $\Omega_{n} \subset S^{n}(\mathcal{A} \backslash B)$ e que $\mathrm{d}_{\mathrm{H}}\left(S^{n}(\mathcal{A} \backslash B), A\right) \leqslant M e^{-\omega n}$, para todo $n$, existe um natural $n_{r}+1>\left(\log \frac{M}{r}\right) / \omega$ tal que

$$
G:=A \cup\left(\bigcup_{n>n_{r}} \Omega_{n}\right) \subset \mathcal{O}_{r}(A) .
$$


Com isso, $N(2 r, G) \leqslant N(r, A)$, pois como podemos cobrir $A$ por $N(r, A)$ bolas centradas em $A$, digamos

$$
A \subset \bigcup_{j=1}^{N(r, A)} B_{X}\left(a_{j}, r\right)
$$

com $a_{j} \in A$, vemos que $\mathcal{O}_{r}(A)$ pode ser coberto pelas $N(r, A)$ bolas $B_{X}\left(a_{j}, 2 r\right)$ :

$$
\mathcal{O}_{r}(A) \subset \bigcup_{j=1}^{N(r, A)} B_{X}\left(a_{j}, 2 r\right)
$$

Agora defina $H:=\bigcup_{n=0}^{n_{r}} \Omega_{n}$, daí

$$
N(r, H) \leqslant\left(n_{r}+1\right) \max _{n=0, \ldots, n_{r}} N\left(r / c^{n}, \Omega_{0}\right)=\left(n_{r}+1\right) N\left(r / c^{n_{r}}, \Omega_{0}\right)
$$

Não é difícil verificar que

$$
\mathcal{A}=B \cup G \cup H \text {. }
$$

De fato, um ponto $x \in \mathcal{A}$ ou pertence a $B$ ou pertence a $\mathcal{A} \backslash B$. No segundo caso, se $x \notin S(\mathcal{A} \backslash B)$, então $x \in \Omega_{0} \subset H$; se $x \in S(\mathcal{A} \backslash B)$, então existe $z_{1} \in \mathcal{A} \backslash B$ tal que $x=S z_{1}$. Neste caso, se $z_{1} \notin S(\mathcal{A} \backslash B)$, então $z_{1} \in \Omega_{0}$ de modo que $x=S z_{1} \in \Omega_{1} \subset H$. Por outro lado, se $z_{1} \in S(\mathcal{A} \backslash B)$, então existe $z_{2} \in \mathcal{A} \backslash B$ tal que $x=S z_{1}=S^{2} z_{2}$. Argumentando como antes, ou $x \in \Omega_{2} \subset H$ ou existe $z_{3} \in \mathcal{A} \backslash B$ tal que $x=S^{3} z_{3}$.

Graças à hipótese $i i)$, este procedimento é finito para todo $x \notin(A \cup B)$, de modo que existe $z_{n} \in \mathcal{A} \backslash B$ tal que $x=S^{n} z_{n} \in \Omega_{n} \subset G \cup H$.

\section{Passo 2: Obter a estimativa}

$$
\operatorname{dim}_{\mathrm{F}}(\mathcal{A}) \leqslant \max \left\{\frac{\omega+\log c}{\omega} \operatorname{dim}_{\mathrm{F}}(B), \operatorname{dim}_{\mathrm{F}}(A)\right\}
$$

Pondo $n:=n_{r}=\left(\log \frac{M}{r}\right) / \omega$ para simplificar a notação, como

$$
\begin{aligned}
N(r, \mathcal{A}) & \leqslant 3 \max \{N(r, B) ; N(r, G) ; N(r, H)\} \\
& \leqslant 3 \max \left\{N(r, B) ; N(r / 2, A) ; n_{r} N\left(r / c^{n_{r}}, \Omega_{0}\right)\right\},
\end{aligned}
$$


segue que

$\frac{\log N(r, \mathcal{A})}{\log 1 / r} \leqslant \frac{\log 3}{\log 1 / r}+\max \left\{\frac{\log N(r, B)}{\log 1 / r} ; \frac{\log N(r / 2, A)}{\log 1 / r} ; \frac{\log n}{\log 1 / r}+\frac{\log N\left(r / c^{n}, \Omega_{0}\right)}{\log 1 / r}\right\}$

Calculemos o limite superior de cada um desses termos.

Os quatro primeiros são simples: $\limsup _{r \rightarrow 0^{+}} \frac{\log 3}{\log 1 / r}=0, \limsup _{r \rightarrow 0^{+}} \frac{\log N(r, B)}{\log 1 / r}=\operatorname{dim}_{\mathrm{F}}(B)$,

$$
\begin{aligned}
\limsup _{r \rightarrow 0^{+}} \frac{\log N(r / 2, A)}{\log 1 / r} & =\limsup _{r \rightarrow 0^{+}} \frac{\log N(r / 2, A)}{\log (2 / 2 r)} \\
& =\limsup _{r \rightarrow 0^{+}} \frac{\log N(r / 2, A)}{\log 2 / r} \frac{1}{1+\frac{\log 1 / 2}{\log 2 / r}} \\
& =\operatorname{dim}_{\mathrm{F}}(A)
\end{aligned}
$$

e

$$
\limsup _{r \rightarrow 0^{+}} \frac{\log n}{\log 1 / r}=\limsup _{r \rightarrow 0^{+}} \frac{\log 1 / \omega}{\log 1 / r}+\limsup _{r \rightarrow 0^{+}} \frac{\log (\log (M / r))}{\log 1 / r}=0 .
$$

Quanto ao último termo, note que $\Omega_{0}=(\mathcal{A} \backslash B) \backslash S(\mathcal{A} \backslash B)$ é subconjunto de $S(B) \backslash B$, de onde segue que

$$
\operatorname{dim}_{\mathrm{F}}\left(\Omega_{0}\right) \leqslant \operatorname{dim}_{\mathrm{F}}(S(B) \backslash B) \leqslant \operatorname{dim}_{\mathrm{F}}(S(B))=\operatorname{dim}_{\mathrm{F}}(B)
$$

E então

$$
\begin{aligned}
\limsup _{r \rightarrow 0^{+}} \frac{\log N\left(r / c^{n}, \Omega_{0}\right)}{\log 1 / r} & =\limsup _{r \rightarrow 0^{+}} \frac{\log N\left(r / c^{n}, \Omega_{0}\right)}{\log c^{n} / r} \frac{1}{1+\frac{\log 1 / c^{n}}{\log c^{n} / r}} \\
& =\operatorname{dim}_{\mathrm{F}}\left(\Omega_{0}\right) \frac{\omega+\log c}{\omega} \\
& \leqslant \operatorname{dim}_{\mathrm{F}}(B) \frac{\omega+\log c}{\omega}
\end{aligned}
$$


pois

$$
\begin{aligned}
\left(1+\frac{\log 1 / c^{n}}{\log c^{n} / r}\right)^{-1} & =\frac{\log c^{n} / r}{\log \left(c^{n} / r\right)+\log 1 / c^{n}} \\
& =\frac{n \log c-\log r}{-\log r} \\
& =1-n \frac{\log c}{\log r} \\
& =1-\frac{\log M / r}{\omega} \frac{\log c}{\log r} \\
& =1-\frac{\log c}{\omega}\left(\frac{\log M-\log r}{\log r}\right) \stackrel{\ln \rightarrow 0^{+}}{\longrightarrow} 1+\frac{\log c}{\omega} .
\end{aligned}
$$

Concluímos que

$$
\operatorname{dim}_{\mathrm{F}}(\mathcal{A}) \leqslant \max \left\{\operatorname{dim}_{\mathrm{F}}(B) ; \operatorname{dim}_{\mathrm{F}}(A) ; \frac{\omega+\log c}{\omega} \operatorname{dim}_{\mathrm{F}}(B)\right\}
$$

como queríamos.

Tendo esta proposição à disposição, podemos estimar a dimensão fractal do atrator de um semigrupo gradiente discreto $\left\{T^{n}: n \in \mathbb{Z}_{+}\right\}$em termos da dimensão fractal dos conjuntos instáveis locais dos invariantes isolados.

A seguir, faremos uso das definições de conjunto instável local (Definição 1.47) e fonte (Definição 1.48), da versão do Lema 1.49 que garante a existência de uma fonte em $\Xi$.

Teorema 4.3. Seja $\left(T^{n}, \Xi\right)$ um semigrupo gradiente discreto em um espaço de Banach $X$ com atrator global $\mathcal{A}$, de modo que cada $W_{\mathrm{loc}}^{u}\left(E_{j}\right)$ é dado como gráfico de uma aplicação Lipschitz; mais precisamente, existe uma decomposição $X=X_{1} \oplus X_{2}, \operatorname{com} \operatorname{dim} X_{1}<\infty$, $U$ uma variedade de $X_{1}$ e uma aplicação Lipschitz $\phi: U \subset X_{1} \rightarrow X_{2}$ de modo que $W_{\mathrm{loc}}^{u}\left(E_{j}\right)=\operatorname{Graf}(\phi)$.

Suponha que $\left.T\right|_{\mathcal{A}}$ seja uma aplicação c-Lipschitz, com $c>1$, e que existam constantes $M \geqslant 1$ e $\omega>0$ tais que

$$
\mathrm{d}_{\mathrm{H}}\left(T^{n}(K), A\right) \leqslant M e^{-\omega n}, \forall n \in \mathbb{Z}_{+},
$$

para todo par atrator-repulsor $\left(A, A^{*}\right)$ e todo compacto $K \subset \mathcal{A}$ disjunto de $A^{*}$. 
Então

$$
\max _{j=1, \ldots, m} \operatorname{dim}_{\mathrm{F}}\left(W_{\mathrm{loc}}^{u}\left(E_{j}\right)\right) \leqslant \operatorname{dim}_{\mathrm{F}}(\mathcal{A}) \leqslant \frac{\omega+\log c}{\omega} \max _{j=1, \ldots, m} \operatorname{dim}_{\mathrm{F}}\left(W_{\mathrm{loc}}^{u}\left(E_{j}\right)\right) .
$$

\section{Demonstração:}

Sejam $E_{j} \in \Xi$ uma fonte e $B_{j}$ uma vizinhança de $E_{j}$ em $\mathcal{A}$ de tal foma que $B_{j} \subset$ $T\left(B_{j}\right) \subset W_{\text {loc }}^{u}\left(E_{j}\right)$. Com isso, $\operatorname{dim}_{\mathrm{F}}\left(B_{j}\right)=\operatorname{dim}_{\mathrm{F}}\left(T\left(B_{j}\right)\right)=\operatorname{dim}_{\mathrm{F}}\left(W_{\text {loc }}^{u}\left(E_{j}\right)\right)$.

De fato, temos a cadeia de desigualdades

$$
\operatorname{dim}_{\mathrm{F}}\left(B_{j}\right) \leqslant \operatorname{dim}_{\mathrm{F}}\left(T\left(B_{j}\right)\right) \leqslant \operatorname{dim}_{\mathrm{F}}\left(B_{j}\right) \leqslant \operatorname{dim}_{\mathrm{F}}\left(W_{\mathrm{loc}}^{u}\left(E_{j}\right)\right)
$$

E também, como $\bigcup_{n=1}^{\infty} T^{n}\left(B_{j}\right)=W^{u}\left(E_{j}\right) \supset W_{\text {loc }}^{u}\left(E_{j}\right)$ é uma reunião crescente (pois $E_{j}$ é fonte), temos que $W_{\text {loc }}^{u}\left(E_{j}\right) \subset \bigcup_{n=1}^{n_{0}} T^{n}\left(B_{j}\right)$, para $n_{0}$ suficientemente grande, então

$$
\operatorname{dim}_{\mathrm{F}}\left(W_{\mathrm{loc}}^{u}\left(E_{j}\right)\right) \leqslant \max _{n=1, \ldots, n_{0}} \operatorname{dim}_{\mathrm{F}}\left(T^{n}\left(B_{j}\right)\right)=\max _{n=1, \ldots, n_{0}} \operatorname{dim}_{\mathrm{F}}\left(B_{j}\right)=\operatorname{dim}_{\mathrm{F}}\left(B_{j}\right),
$$

e isto basta.

Note ainda que $E_{j}=A_{j}^{*}$, sendo $A_{j}=\bigcup_{k \neq j} W_{\text {loc }}^{u}\left(E_{k}\right)$.

Pela proposição acima,

$$
\operatorname{dim}_{\mathrm{F}}\left(B_{j}\right) \leqslant \operatorname{dim}_{\mathrm{F}}(\mathcal{A}) \leqslant \max \left\{\frac{\omega+\log c}{\omega} \operatorname{dim}_{\mathrm{F}}\left(B_{j}\right) ; \operatorname{dim}_{\mathrm{F}}\left(A_{j}\right)\right\}
$$

isto é,

$$
\operatorname{dim}_{\mathrm{F}}\left(W_{\text {loc }}^{u}\left(E_{j}\right)\right) \leqslant \operatorname{dim}_{\mathrm{F}}(\mathcal{A}) \leqslant \max \left\{\frac{\omega+\log c}{\omega} \operatorname{dim}_{\mathrm{F}}\left(W_{\mathrm{loc}}^{u}\left(E_{j}\right)\right) ; \operatorname{dim}_{\mathrm{F}}\left(A_{j}\right)\right\} .
$$

Agora restringimos o operador $T$ ao atrator local $A_{j}$. Portanto temos um semigrupo discreto gradiente com atrator $A$ e $\boldsymbol{\Xi}^{\mathbf{1}}=\boldsymbol{\Xi} \backslash\left\{E_{j}\right\}$, o que tem pelo menos uma fonte $\Xi_{k}$, com $k \neq i$. Podemos usar o mesmo argumento acima para provar que

$$
\operatorname{dim}_{\mathrm{F}}\left(W_{\mathrm{loc}}^{u}\left(\Xi_{k}\right)\right) \leqslant \operatorname{dim}_{\mathrm{F}}\left(A_{i}\right) \leqslant \max \left\{\frac{\omega+\log (c)}{\omega} \operatorname{dim}_{\mathrm{F}}\left(W_{\mathrm{loc}}^{u}\left(\Xi_{k}\right)\right), \operatorname{dim}_{\mathrm{F}}\left(A_{k}\right)\right\} .
$$


E usando estes dois resultados, obtemos que $\operatorname{dim}_{\mathrm{F}}(\mathcal{A})$ é limitada inferiormente por

$$
\max _{j=i, k} \operatorname{dim}_{\mathrm{F}}\left(W_{\mathrm{loc}}^{u}\left(\Xi_{j}\right)\right)
$$

e superiormente por

$$
\max \left\{\frac{\omega+\log (c)}{\omega} \operatorname{dim}_{\mathrm{F}}\left(W_{\mathrm{loc}}^{u}\left(E_{j}\right)\right), \frac{\omega+\log (c)}{\omega} \operatorname{dim}_{\mathrm{F}}\left(W_{\mathrm{loc}}^{u}\left(\Xi_{k}\right)\right), \operatorname{dim}_{\mathrm{F}}\left(A_{k}\right)\right\} .
$$

Este processo deve parar pois existem somente finitos conjuntos invariantes isolados e procedendo indutivamente obtemos o resultado desejado.

A demonstração desse teorema sugere que, após uma permutação na F.F.D.I.I. $\Xi$, podemos assumir $\Xi=\left\{E_{1}, \ldots, E_{m}\right\}$ é ordenada de tal forma que $E_{1}$ é uma fonte em $\mathcal{A}$, $E_{2}$ é uma fonte em $A_{1}$ para a restrição de $T$ e, tendo definido $E_{j-1}$, o invariante $E_{j}$ é uma fonte para a restrição de $T$ a $A_{k}=\bigcup_{j=k}^{m} W^{u}\left(E_{j}\right)$.

Com esta reordenação, formamos uma nova família $\mathcal{N}=\left\{\mathcal{N}_{1}, \ldots, \mathcal{N}_{p}\right\}$, com $p \leqslant m$, que é uma Decomposição de Morse para $\mathcal{A}$ - a chamada decomposição por níveis de energia de $\mathcal{A}$. Para mais detalhes veja, [1].

Por meio desta decomposição, podemos ver que a dimensão fractal dos conjuntos $W_{\text {loc }}^{u}\left(E_{j}\right)$ é uma função decrescente do índice $i$, e obtemos que

$$
\operatorname{dim}_{\mathrm{F}}\left(W_{\mathrm{loc}}^{u}\left(\Xi_{1}\right)\right) \leqslant \operatorname{dim}_{\mathrm{F}}(\mathcal{A}) \leqslant \frac{\omega+\log (c)}{\omega} \operatorname{dim}_{\mathrm{F}}\left(W_{\mathrm{loc}}^{u}\left(\Xi_{1}\right)\right)
$$

Consulte Carvalho [8] para resultados particularizados para semigrupos gradientes com pontos fixos hiperbólicos.

Corolário 4.4. Sejam $\left\{T^{n}: n \in \mathbb{Z}_{+}\right\}$um semigrupo gradiente discreto com atrator global $\mathcal{A}, \mathcal{E}=\left\{e_{1}, \ldots, e_{m}\right\}$ um conjunto de pontos fixos e $A_{i}:=\bigcup_{j \neq i} W_{\mathrm{loc}}^{u}\left(e_{j}\right)$.

Suponha que $\left.T\right|_{\mathcal{A}}$ seja uma aplicação c-Lipschitz, com $c>1$, e que existam constantes $M \geqslant 1$ e $\omega>0$ tais que

$$
\mathrm{d}_{\mathrm{H}}\left(T^{n}(K), A\right) \leqslant M e^{-\omega n}, \forall n \in \mathbb{Z}_{+},
$$


para todo par atrator-repulsor $\left(A, A^{*}\right)$ e todo compacto $K \subset \mathcal{A}$ disjunto de $A^{*}$.

Então,

$$
\max _{i=1, \ldots, m} \operatorname{dim}_{\mathrm{F}}\left(W_{\mathrm{loc}}^{u}\left(e_{i}\right)\right) \leqslant \operatorname{dim}_{\mathrm{F}}(\mathcal{A}) \leqslant \frac{\omega+\log (c)}{\omega} \max _{i=1, \ldots, m} \operatorname{dim}_{\mathrm{F}}\left(W_{\mathrm{loc}}^{u}\left(e_{i}\right)\right) .
$$

Corolário 4.5. Nas condições do Teorema 4.3, o atrator global pode ser projetado injetivamente em $\mathbb{R}^{N}$, para todo natural $N$ suficientemente grande.

\subsection{Atratores Exponenciais}

Sabemos que o estudo da dinâmica futura de um sistema dinâmico pode ser reduzido ao estudo da dinâmica no atrator global, que é o menor compacto invariante que atrai todos os conjuntos limitados do espaço de fase. Acontece que o atrator é, em geral, sensível a perturbações e a taxa de convergência com que os conjuntos limitados são atraídos pode ser arbitrariamente lenta.

Para contornar tais obstáculos, surge o conceito de atrator exponencial, que ainda demanda atração exponencial dos limitados do espaço, mas enfraquece a propriedade de invariância, o que custa a unicidade deste objeto. Os resultados que apresentamos são baseados em [8], [9] e [13], e se propõem a estimar a dimensão fractal dos atratores exponenciais e consequentemente a do atrator global para semigrupos discretos, pois este é, em geral, menor que aqueles. O caso contínuo e outras generalizações podem ser consultados em [9].

Uma vez que a finitude já é suficiente para os nossos propósitos, não existe a preocupação em obter as melhores cotas superiores para a dimensão fractal dos atratores exponenciais. Com isso, tais estimativas dependem das propriedades de regularização do operador e das propriedades de imersões compactas entre espaços de Banach.

A construção de atratores exponenciais apresentada em [13] serve de base para [9], e consequentemente para o que apresentamos aqui. Além disso, contém exemplos de como os resultados abstratos de atratores exponenciais podem ser aplicados a diversos problemas matemáticos.

Definição 4.6. Seja $\left\{T^{n}: n \in \mathbb{Z}_{+}\right\}$um semigrupo discreto em um espaço métrico $X$.

Dizemos que $\mathcal{M} \subset X$ é um atrator exponencial para $\left\{T^{n}: n \in \mathbb{Z}_{+}\right\}$se

i) $\mathcal{M}$ é compacto e positivamente invariante, i.e., $T^{n}(\mathcal{M}) \subset \mathcal{M}$, para todo $n$; 
ii) $\operatorname{dim}_{\mathrm{F}}(\mathcal{M})$ é finita;

iii) existe $\gamma>0$ tal que, para todo conjunto limitado $B \subset X$,

$$
\lim _{n \rightarrow \infty} e^{\gamma n} \operatorname{dist}_{H}\left(T^{n}(B), \mathcal{M}\right)=0
$$

A constante $\gamma$ na Definição 4.6 é chamada de taxa de atração de $\mathcal{M}$ e a ela devemos a propriedade de maior robustez que os atratores exponenciais têm em relação ao atrator global (veja [9]).

Note que se $\mathcal{M}$ é um atrator exponencial, então $\mathcal{M}_{t}:=T(t) \mathcal{M}$ é um atrator exponencial, para todo $t>0$ real.

A seguir enunciamos condições a partir das quais asseguramos a existência de uma família de atratores exponenciais $\mathcal{M}_{\nu}$ de dimensão fractal finita, com $\nu \in(0,1)$. Também segue a existência de um atrator global $\mathcal{A} \subset \mathcal{M}_{\nu}$, o qual possui dimensão fractal finita por consequência.

Considere as hipóteses

H1) sejam $\left(X,\|\cdot\|_{X}\right)$ e $\left(Y,\|\cdot\|_{Y}\right)$ espaços de Banach, em que $X$ está compactamente imerso em $Y$;

H2) $T: X \rightarrow X$ uma aplicação contínua tal que $\left\{T^{n}: n \in \mathbb{Z}_{+}\right\}$é um semigrupo discreto limitado dissipativo, isto é, existe $B_{0} \subset X$ limitado tal que para cada conjunto limitado $B \subset X$, existe $n_{B} \in \mathbb{N}$ para o qual vale

$$
T^{n}(B) \subset B_{0}, \forall n \geq n_{B}
$$

H3) existe $K>0$ tal que

$$
\|T x-T y\|_{X} \leqslant K\|x-y\|_{Y}, \forall x, y \in B_{0} .
$$

Da hipótese $H 1$ ), vemos que os subconjuntos limitados de $X$ são subconjuntos totalmente limitados de $Y$, o que em certo sentido diz que a topologia de $Y$ é mais fraca que a de $X$. Dado $\nu \in(0,1)$, como $B_{0} \subset X$ e $B_{X}(0,1) \subset X$ são pré-compactos em $\left(Y,\|\cdot\|_{Y}\right)$, podemos cobri-los por um número finito de bolas de $Y$ de raios $\nu / K$ e $\nu / 2 K$, 
respectivamente:

$$
N_{0}=N\left(\frac{\nu}{K}, B_{0}\right) \text { e } N_{\nu}=N\left(\frac{\nu}{2 K}, B_{X}(0,1)\right)
$$

A ideia da demonstração do próximo teorema é usar recursivamente o seguinte princípio

$$
B \subset X \text { limitado } \Rightarrow B \subset Y \text { totalmente limitado } \stackrel{\text { por } H 3)}{\Rightarrow} T(B) \subset X \text { limitado. }
$$

Teorema 4.7. Se valem as hipóteses H1),H2) e H3), então o semigrupo $\left\{T^{n}: n \in \mathbb{Z}_{+}\right\}$ possui um atrator global $\mathcal{A}$ e, para cada $\nu \in(0,1)$, possui um atrator exponencial $\mathcal{M}_{\nu}$ com

$$
\operatorname{dim}_{\mathrm{F}}(\mathcal{A}) \leqslant \operatorname{dim}_{\mathrm{F}}\left(\mathcal{M}_{\nu}\right) \leqslant \frac{\log N_{\nu}}{\log 1 / \nu}
$$

Demonstração: A menos de tomar iteradas de $T$, podemos assumir que $n_{B_{0}}=1$, ou seja, vamos assumir que $T\left(B_{0}\right) \subset B_{0}$.

Fixado $\nu \in(0,1)$, podemos escolher $V_{0}=\left\{x_{1}, x_{2}, \ldots, x_{N_{0}}\right\} \subset B_{0}$ tal que

$$
B_{0} \subset \bigcup_{i=1}^{N_{0}} B_{Y}\left(x_{i}, \nu / K\right)
$$

de modo que

$$
T\left(B_{0}\right)=\bigcup_{i=1}^{N_{0}} B_{X}\left(T x_{i}, \nu\right) \cap T\left(B_{0}\right)
$$

pois, dado $x \in B_{0}$, tem-se $\left\|T x-T x_{i_{0}}\right\|_{X} \leqslant K\left\|x-x_{i_{0}}\right\|_{Y}<\nu$, para algum $i_{0}$.

Como a bola unitária $B_{X}(0,1)$ pode ser coberta pela reunião $\bigcup_{j=1}^{N_{\nu}} B_{Y}\left(y_{j}, \nu / 2 K\right)$, para alguma escolha de pontos $y_{j} \in B_{X}(0,1)$.

Por linearidade, obtemos a inclusão $B_{X}\left(T x_{i}, \nu\right) \subset \bigcup_{j=1}^{N_{\nu}} B_{Y}\left(y_{j}+T x_{i}, \nu^{2} / 2 K\right)$ e de (4.2.1) segue que

$$
T\left(B_{0}\right)=\bigcup_{i=1}^{N_{0}} \bigcup_{j=1}^{N_{\nu}} B_{Y}\left(y_{j}+T x_{i}, \nu^{2} / 2 K\right) \cap T\left(B_{0}\right) .
$$

Assim, fixando pontos $x_{i, j} \in B_{Y}\left(y_{j}+T x_{i}, \nu^{2} / 2 K\right) \cap T\left(B_{0}\right)$, com $1 \leqslant i \leqslant N_{0}$ e $1 \leqslant j \leqslant N_{\nu}$, 
obtemos

$$
T\left(B_{0}\right)=\bigcup_{i=1}^{N_{0}} \bigcup_{j=1}^{N_{\nu}} B_{Y}\left(x_{i, j}, \nu^{2} / K\right) \cap T\left(B_{0}\right) .
$$

Seja $V_{1} \subset T\left(B_{0}\right)$ o conjunto de tais pontos $x_{i, j}$.

Com isso, existem pontos $x_{i, j, k} \in T^{2}\left(B_{0}\right)$, com $1 \leqslant i \leqslant N_{0}$ e $1 \leqslant j, k \leqslant N_{\nu}$, de tal forma que

$$
\begin{aligned}
T^{2}\left(B_{0}\right) & =\bigcup_{i=1}^{N_{0}} \bigcup_{j=1}^{N_{\nu}} T\left(B_{Y}\left(x_{i, j}, \nu^{2} / K\right)\right) \cap T^{2}\left(B_{0}\right) \\
& =\bigcup_{i=1}^{N_{0}} \bigcup_{j=1}^{N_{\nu}} B_{X}\left(T x_{i, j}, \nu^{2}\right) \cap T^{2}\left(B_{0}\right) \\
& =\bigcup_{i=1}^{N_{0}} \bigcup_{j=1}^{N_{\nu}} \bigcup_{k=1}^{N_{\nu}} B_{Y}\left(x_{i, j, k}, \nu^{3} / K\right) \cap T^{2}\left(B_{0}\right)
\end{aligned}
$$

e consequentemente

$$
T^{3}\left(B_{0}\right)=\bigcup_{x \in V_{2}} B_{X}\left(T x, \nu^{3}\right) \cap T^{3}\left(B_{0}\right)
$$

em que $V_{2} \subset T^{2}\left(B_{0}\right)$ denota o conjunto dos pontos $x_{i, j, k}$.

Procedendo por indução, obtemos $V_{n} \subset T^{n}\left(B_{0}\right)$, com $\# V_{n}=N_{0} N_{\nu}^{n}$, de modo que

$$
T^{n}\left(B_{0}\right) \subset \bigcup_{x \in V_{n-1}} B_{X}\left(T x, \nu^{n}\right)
$$

Em particular, $\operatorname{dist}_{\mathrm{H}}\left(T^{n}\left(B_{0}\right), T\left(V_{n-1}\right)\right) \leqslant \nu^{n}$ e $T\left(B_{0}\right) \subset X$ é pré-compacto de $X$, consequentemente, $\left\{T^{n}: n \in \mathbb{Z}_{+}\right\}$é um semigrupo assintoticamente compacto. Como já tínhamos a dissipatividade pela hipótese $H 2)$, do Teorema ?? segue que $\left\{T^{n}: n \in \mathbb{Z}_{+}\right\}$ possui um atrator global $\mathcal{A}$.

Uma vez que $\mathcal{A}$ é invariante e é absorvido por $B_{0}$, segue que $\mathcal{A} \subset T^{n}\left(B_{0}\right)$, para todo 
$n ; \operatorname{assim} N\left(\nu^{n}, \mathcal{A}\right) \leqslant N_{0} N_{\nu}^{n-1}$ por (4.2.2), o que implica que

$$
\begin{aligned}
\operatorname{dim}_{\mathrm{F}}(\mathcal{A}) & =\limsup _{n \rightarrow \infty} \frac{\log N\left(\nu^{n}, \mathcal{A}\right)}{\log 1 / \nu^{n}} \\
& \leqslant \limsup _{n \rightarrow \infty}\left(\frac{\log N_{0}}{n \log 1 / \nu}+\frac{n-1}{n} \frac{\log N_{\nu}}{\log 1 / \nu}\right) \\
& =\frac{\log N_{\nu}}{\log 1 / \nu}<\infty .
\end{aligned}
$$

Agora, considere os conjuntos $E_{0}=V_{0}, E_{n+1}=V_{n+1} \cup T\left(E_{n}\right), n=0,1,2, \ldots$ e seja

$$
\mathcal{M}_{\nu}={\overline{\bigcup_{n \geq 0} E_{n}}}^{X}
$$

o que indica que o fecho é tomado considerando a topologia de $\left(X,\|\cdot\|_{X}\right)$.

Da continuidade da aplicação $T^{n}: X \rightarrow X$ e do fato de $E_{n+1} \supset T\left(E_{n}\right)$ segue que $T^{n}\left(\mathcal{M}_{\nu}\right) \subset \mathcal{M}_{\nu}$, para todo $n$; logo, $\mathcal{M}_{\nu}$ é positivamente invariante pela ação de $T$ e vale $T\left(V_{n-1}\right) \subset T\left(\mathcal{M}_{\nu}\right) \subset \mathcal{M}_{\nu}$.

Quanto à atração exponencial, dado $B \subset X$ limitado, temos que $T^{n+n_{B}}(B) \subset T^{n}\left(B_{0}\right)$, para todo $n$, e então

$$
\begin{aligned}
\operatorname{dist}_{\mathrm{H}}\left(T^{n+n_{B}}(B), \mathcal{M}_{\nu}\right) & \leqslant \operatorname{dist}_{\mathrm{H}}\left(T^{n+n_{B}}(B), T\left(V_{n-1}\right)\right) \\
& \leqslant \operatorname{dist}_{\mathrm{H}}\left(T^{n}\left(B_{0}\right), T\left(V_{n-1}\right)\right) \\
& \leqslant \nu^{-n_{B}} \nu^{n+n_{B}} .
\end{aligned}
$$

Em outras palavras, pondo $k=\nu^{-n_{B}}$, vale

$$
\operatorname{dist}_{\mathrm{H}}\left(T^{m}(B), \mathcal{M}_{\nu}\right) \leqslant k \nu^{m}=k e^{m \log \nu}, \forall m>n_{B}
$$

e então basta considerar $\gamma=-(1 / 2) \log \nu$.

Para verificar que $\mathcal{M}_{\nu}$ é compacto, note que, por construção, $E_{0}$ é finito e $E_{n} \subset T\left(B_{0}\right)$ para cada $n$, de modo que $\mathcal{M}_{\nu}$ é um subconjunto fechado de $\overline{T\left(B_{0}\right)} \times \cup{\overline{E_{0}}}^{X}$, o qual é compacto.

Por fim, mostremos a limitação superior $\operatorname{de} \operatorname{dim}_{\mathrm{F}}\left(\mathcal{M}_{\nu}\right)$. 
Não é difícil verificar que $E_{n+j} \subset T^{n}\left(B_{0}\right)$, para todos os $n, j \in \mathbb{N}$; assim

$$
\mathcal{M}_{\nu} \subset E_{0} \cup E_{1} \cup \ldots \cup E_{n} \cup \overline{T^{n}\left(B_{0}\right)}
$$

Agora, \# $\left(E_{0} \cup \ldots \cup E_{n}\right) \leqslant\left((n+1)^{2}-1\right) N_{0} N_{\nu}^{n-1}$ e $N\left(\nu^{n}, T^{n}\left(B_{0}\right)\right) \leqslant N_{0} N_{\nu}^{n-1}$ implicam que $N\left(\nu^{n}, \mathcal{M}_{\nu}\right) \leqslant(n+1)^{2} N_{0} N_{\nu}^{n-1}$ e então

$$
\begin{aligned}
\operatorname{dim}_{\mathrm{F}}\left(\mathcal{M}_{\nu}\right) & =\limsup _{n \rightarrow \infty} \frac{\log N\left(\nu^{n}, \mathcal{M}_{\nu}\right)}{\log 1 / \nu^{n}} \\
& \leqslant \limsup _{n \rightarrow \infty}\left(\frac{2 \log (n+1)}{n \log 1 / \nu}+\frac{\log N_{0}}{n \log 1 / \nu}+\frac{n-1}{n} \frac{\log N_{\nu}}{\log 1 / \nu}\right) \\
& =\frac{\log N_{\nu}}{\log 1 / \nu} .
\end{aligned}
$$

Agora, podemos ver como o Lema 2.33 é fundamental para calcular a dimensão fractal de $\mathcal{M}_{\nu}$ usando diferentes maneiras de fazer o raio das bolas tender a 0 .

Lembrando que um semigrupo $\left\{T^{n}: n \in \mathbb{Z}_{+}\right\}$possui atrator global se, e somente se, é assintoticamente compacto e limitado dissipativo, a partir da demonstração do Teorema 4.7, obtemos o seguinte resultado.

Corolário 4.8. Sejam $X$ e $Y$ espaços de Banach tais que $X$ está compactamente imerso em $Y$. Se $\left\{T^{n}: n \in \mathbb{Z}_{+}\right\}$é um semigrupo com atrator global $\mathcal{A}$ e existe $K>0$ tal que

$$
\|T x-T y\|_{X} \leqslant K\|x-y\|_{Y}
$$

para todos os $x, y \in \mathcal{A}$, então $\operatorname{dim}_{\mathrm{F}}(\mathcal{A})<\infty$.

As hipóteses H2) e H3) do Teorema 4.7 implicitamente impuseram que o semigrupo associado a $T: X \rightarrow X$ possuísse um atrator global, uma vez que $T\left(B_{0}\right)$ é um conjunto absorvente compacto. Agora vamos enfraquecer esta hipótese pedindo que $T$ seja uma decomposição de um operador compacto perturbado por um operador linear contínuo que é bem "controlado". Assumindo sua existência, o atrator global possui dimensão fractal finita.

Considere as hipóteses 
H1) $\operatorname{sejam}\left(X,\|\cdot\|_{X}\right)$ e $\left(Y,\|\cdot\|_{Y}\right)$ espaços de Banach, em que $X$ está compactamente imerso em $Y$;

H2) o semigrupo associado a $T: X \rightarrow X$ possui atrator global $\mathcal{A}$;

H3) $T$ admite a decomposição $T=L+C$, em que $L, C: X \rightarrow X$ são também contínuas, e existem $\lambda \in(0,1 / 2)$ e $K>0$ tais que, para todos os $x, y \in \mathcal{A}$, valem

$$
\|L x-L y\|_{X} \leqslant \lambda\|x-y\|_{X}
$$

$\mathrm{e}$

$$
\|C x-C y\|_{X} \leqslant K\|x-y\|_{Y}
$$

H4) existe um conjunto limitado absorvente $B_{0} \subset X$ tal que $T\left(B_{0}\right) \subset B_{0}$ e valem as estimativas (4.2.3) e (4.2.4) para todos os pontos $x, y \in B_{0}$.

Novamente, a hipótese $H 1$ ) nos diz que os subconjuntos limitados de $X$ são subconjuntos totalmente limitados de $Y$, de modo que, dado $\nu \in(0,1 / 2-\lambda)$, a bola $B_{X}(0,1) \subset X$ requer $N_{\nu}$ bolas de $Y$ de raio $\nu / K$ para ser coberta:

$$
N_{\nu}=N\left(\frac{\nu}{K}, B_{X}(0,1)\right)
$$

Teorema 4.9. Suponha válidas as hipóteses H1), H2) e H3), e fixe $\nu \in(0,1 / 2-\lambda)$. Então vale a estimativa

$$
\operatorname{dim}_{\mathrm{F}}(\mathcal{A}) \leqslant \frac{\log N_{\nu}}{\log \left(\frac{1}{2(\lambda+\nu)}\right)} .
$$

Além disso, se vale $H 4)$, então existe um atrator exponencial $\mathcal{M}_{\nu}$ para $\left\{T^{n}: n \in \mathbb{Z}_{+}\right\}$ tal que

$$
\operatorname{dim}_{\mathrm{F}}\left(\mathcal{M}_{\nu}\right) \leqslant \frac{\log N_{\nu}}{\log \left(\frac{1}{2(\lambda+\nu)}\right)}
$$

Demonstração: Seja $0<\nu<1 / 2-\lambda$.

Da compacidade de $\mathcal{A}$ em $X$, podemos cobri-lo por $N_{1}$ bolas de $X$ de raio $2(\lambda+\nu)$ centradas em pontos de $\mathcal{A}$ :

$$
\mathcal{A}=\bigcup_{i=1}^{N_{1}} B_{X}\left(x_{i}, 2(\lambda+\nu)\right) \cap \mathcal{A},
$$


para alguma escolha de pontos $x_{1}, \ldots, x_{N_{1}} \in \mathcal{A}$; e agora pela invariância de $\mathcal{A}$,

$$
\begin{aligned}
\mathcal{A}=T(\mathcal{A}) & =\bigcup_{i=1}^{N_{1}}\left(L\left(B_{X}\left(x_{i}, 2(\lambda+\nu)\right) \cap \mathcal{A}\right)+C\left(B_{X}\left(x_{i}, 2(\lambda+\nu)\right) \cap \mathcal{A}\right)\right) \\
& =\bigcup_{i=1}^{N_{1}}\left(B_{X}\left(L x_{i}, 2 \lambda(\lambda+\nu)\right)+\bigcup_{j=1}^{N_{\nu}} B_{X}\left(y_{i, j}, 2 \nu(\lambda+\nu)\right)\right) \cap \mathcal{A} \\
& =\bigcup_{i=1}^{N_{1}} \bigcup_{j=1}^{N_{\nu}} B_{X}\left(L x_{i}+y_{i, j}, 2(\lambda+\nu)^{2}\right) \cap \mathcal{A},
\end{aligned}
$$

para alguma escolha de $\left\{y_{i, j}: 1 \leqslant i \leqslant N_{1}, 1 \leqslant j \leqslant N_{\nu}\right\}$ em $X$.

Procedendo como no teorema anterior, existem pontos $x_{i, j}$ em $\mathcal{A}$, com $1 \leqslant i \leqslant N_{1}, 1 \leqslant$ $j \leqslant N_{\nu}$, tais que

$$
\mathcal{A}=\bigcup_{i=1}^{N_{1}} \bigcup_{j=1}^{N_{\nu}} B_{X}\left(x_{i, j}, 2^{2}(\nu+\lambda)^{2}\right) \cap \mathcal{A}
$$

e assim, obtemos para cada $n \in \mathbb{N}$ um conjunto $V_{n} \subset \mathcal{A}$ tal que $\# V_{n}=N_{1} N_{\nu}^{n-1}$ e

$$
\mathcal{A}=\bigcup_{x \in V_{n}} B_{X}\left(x, 2^{n}(\nu+\lambda)^{n}\right) \cap \mathcal{A}
$$

Com isso,

$$
\operatorname{dim}_{\mathrm{F}}(\mathcal{A}) \leqslant \frac{\log N_{\nu}}{\log \left(\frac{1}{2(\nu+\lambda)}\right)}
$$

o que encerra a primeira parte da demonstração.

Agora suponha válida a hipótese $H 4)$. Uma vez que $B_{0} \subset B_{X}(0, R)$, para algum raio $R>0$, segue de (4.2.3) e (4.2.4) que

$$
T\left(B_{0}\right)=\bigcup_{i=1}^{N_{\nu}} B_{X}\left(x_{i}, 2(\lambda+\nu) R\right) \cap T\left(B_{0}\right),
$$

para alguma escolha de pontos $x_{i} \in T\left(B_{0}\right)$. 
Disso, obtemos que

$$
\begin{aligned}
T^{2}\left(B_{0}\right) & \subset \bigcup_{i=1}^{N_{\nu}}(L+C)\left(B_{X}\left(x_{i}, 2(\lambda+\nu) R\right) \cap T\left(B_{0}\right)\right) \\
& \subset \bigcup_{i=1}^{N_{\nu}}\left(B_{X}\left(L x_{i}, 2 \lambda(\lambda+\nu) R\right)+\bigcup_{j=1}^{N_{\nu}} B_{X}\left(y_{i, j}, 2 \nu(\lambda+\nu) R\right)\right) \\
& =\bigcup_{i=1}^{N_{\nu}} \bigcup_{j=1}^{N_{\nu}} B_{X}\left(L x_{i}+y_{i, j}, 2(\lambda+\nu)^{2} R\right)
\end{aligned}
$$

para alguma escolha de pontos $y_{i, j}$ em $X$. Basta então considerar pontos $x_{i, j}$ em $T^{2}\left(B_{0}\right)$, com $1 \leqslant i, j \leqslant N_{\nu}$, de tal modo que

$$
T^{2}\left(B_{0}\right)=\bigcup_{i=1}^{N_{\nu}} \bigcup_{j=1}^{N_{\nu}} B_{X}\left(x_{i, j}, 2^{2}(\lambda+\nu)^{2} R\right) \cap T^{2}\left(B_{0}\right)
$$

Seguindo esta construção, obtemos um conjunto $V_{n} \subset T^{n}\left(B_{0}\right)$ com $\# V_{n}=N_{\nu}^{n}$ e tal que

$$
T^{n}\left(B_{0}\right)=\bigcup_{x \in V_{n}} B_{X}\left(x, 2^{n}(\lambda+\nu)^{n} R\right) \cap T^{n}\left(B_{0}\right) .
$$

Em particular, $\operatorname{dist}_{\mathrm{H}}\left(T^{n}\left(B_{0}\right), V_{n}\right) \leqslant 2^{n}(\lambda+\nu)^{n} R$. Como antes, defina

$$
\mathcal{M}_{\nu}=\varlimsup_{n \geq 0} E_{n}{ }^{X}
$$

em que $E_{0}=V_{0}:=\left\{b_{0}\right\}$ e $E_{n+1}=V_{n+1} \cup T\left(E_{n}\right)$, para $n=0,1,2 \ldots$

Assim, $\mathcal{M}_{\nu}$ é um compacto positivamente invariante e atrai exponencialmente os limitados de $X$ com constante $\gamma=(1 / 2) \log \left(\frac{1}{2(\lambda+\nu)}\right)$.

Finalmente,

$$
\mathcal{M}_{\nu} \subset E_{0} \cup E_{1} \cup \ldots \cup E_{n-1} \cup \overline{T^{n}\left(B_{0}\right)},
$$

$\#\left(E_{0} \cup \ldots \cup E_{n-1}\right) \leqslant\left((n+1)^{2}-1\right) N_{\nu}^{n-1}$ e $N\left((2(\lambda+\nu))^{n} R, T^{n}\left(B_{0}\right)\right) \leqslant N_{\nu}^{n}$ implicam que

$$
\operatorname{dim}_{\mathrm{F}}\left(\mathcal{M}_{\nu}\right) \leqslant-\frac{\log N_{\nu}}{\log 2(\lambda+\nu)}
$$

como queríamos. 
Não há nada de especial em pedir $0<\lambda<1 / 2$, senão por razões técnicas, como foi visto na demonstração acima. Com isso, a menos de trabalhar com iteradas de $L$, podemos pedir $0<\lambda<1$.

Corolário 4.10. Suponha válidas as hipóteses H1), H2) e H3).

Para cada $\nu \in(0,1 / 2-\lambda)$, o atrator global $\mathcal{A}$ do semigrupo discreto $\left\{T^{n}: n \in \mathbb{Z}_{+}\right\}$pode ser projetado injetivamente em $\mathbb{R}^{N}$, para qualquer natural $N \geqslant 2\left(\frac{\log N_{\nu}}{\log \left(\frac{1}{2(\lambda+\nu)}\right)}\right)+1$.

\subsection{Compactos Negativamente Invariantes}

Seguindo Carvalho [8] e [23], esta seção se dedica a corrigir e a melhorar os resultados apresentados em Mañé [24] e em Hale [19] sobre a dimensão fractal de conjuntos compactos negativamente invariantes. Assim, concluímos o estudo sobre os atratores globais ao verificar que eles têm, em geral, dimensão fractal finita e então o Corolário 3.7 cumpre seu papel: tais atratores são objetos de dimensão finita.

Basicamente, aplicações $T: X \rightarrow X$ tais que sua derivada pode ser decomposta como um operador compacto de posto finito perturbado por um contínuo "pequeno" geram semigrupos cujos atratores, se existem, têm dimensão fractal finita. As principais referências são [7], [8], [23] e [28].

Vamos proceder conforme o seguinte roteiro:

1) mostrar que espaços de dimensão $m$ admitem uma base de Auerbach;

2) mostrar que a distância de Banach-Mazur entre um tal espaço e $\mathbb{R}^{m}$ é no máximo $\log m$;

3) estimar $N\left(\rho, B_{X}(0, r)\right)$ em termos de $m=\operatorname{dim}(X), r$ e $\rho$;

4) mostrar que certas aplicações $T$ satisfazem $T\left(B_{X}(0,1)\right) \subset \mathcal{O}_{\lambda}\left(T\left(B_{Y}(0,1)\right)\right)$, para algum subespaço $Y$ finito dimensional;

5) por fim, estabelecer critérios sobre $T: X \rightarrow X$ de modo que um compacto $K$ negativamente invariante satisfaça

$$
\operatorname{dim}_{\mathrm{F}}(K) \leqslant-\frac{\log M}{\log \alpha}
$$

para constantes convenientes $M$ e $\alpha$. 
Feito isto, vamos aplicar o resultado para $K=\mathcal{A}$ e obter a estimativa acima para sua dimensão fractal. Em [7], os autores consideram uma larga classe de equações diferenciais parciais parabólicas (semilineares em espaços de Banach), as quais detêm atratores globais finito dimensionais.

Vamos nos restringir a estudar espaços vetoriais sobre $\mathbb{R}$, uma vez que espaços complexos pouco acrescentam tanto em resultados como em dificuldades para demonstrá-los. Mas vale apontar diferenças quando não forem óbvias.

Lema 4.11. Seja $X=\left(X,\|\cdot\|_{X}\right)$ um espaço vetorial normado sobre $\mathbb{R}$ de dimensão $m$. Então, existem bases $\left\{x_{1}, \cdots, x_{m}\right\}$ de $X$ e $\left\{x_{1}^{*}, \cdots, x_{m}^{*}\right\}$ de $X^{*}$ tais que, para todos os $1 \leqslant i, j \leqslant m$,

$$
\left\|x_{i}\right\|_{X}=\left\|x_{j}^{*}\right\|_{X^{*}}=1 \text { e }<x_{j}^{*}, x_{i}>=\delta_{i, j} .
$$

Neste caso, tal par de bases é chamado uma base de Auerbach para X.

Demonstração: Seja $\mathcal{B}=\left(v_{1}, \ldots, v_{m}\right)$ uma base ordenada de $X$ e, para cada $z \in X$, seja $\hat{z}$ o vetor coluna cujas entradas são as coordenadas de $z$ na base $\mathcal{B}$.

Considere a aplicação contínua

$$
\begin{aligned}
& f: \quad X^{m} \quad \longrightarrow \mathbb{R} \\
& \left(x_{1}, \ldots, x_{m}\right) \longmapsto f\left(x_{1}, \ldots, x_{m}\right):=\operatorname{det}\left[\hat{x}_{1}, \cdots, \hat{x}_{m}\right]
\end{aligned}
$$

e denote por $B$ a bola fechada unitária de $X$, de modo que $B^{m} \subset X^{m}$ é um compacto. Com isso, existe $\left(x_{1}, \ldots, x_{m}\right) \in B^{m}$ em que $|f|$ assume seu máximo $a>0$, uma vez que $f\left(\frac{v_{1}}{\left\|v_{1}\right\|_{X}}, \ldots, \frac{v_{m}}{\left\|v_{m}\right\|_{X}}\right) \neq 0$

Afirmo que $\left\|x_{i}\right\|_{X}=1$, para todo $i=1, \ldots, m$. Caso contrário, $\left\|x_{i_{0}}\right\|_{X}<1$, para algum $i_{0}$; pondo $z_{i_{0}}:=\frac{1}{\left\|x_{i_{0}}\right\|_{X}} x_{i_{0}}$, temos

$$
\left|f\left(x_{1}, \ldots, x_{i_{0}-1}, z_{i_{0}}, x_{i_{0}+1}, \ldots, x_{m}\right)\right|=\frac{1}{\left\|x_{i_{0}}\right\|_{X}}\left|f\left(x_{1}, \ldots, x_{m}\right)\right|>a
$$

o que é uma contradição.

Em particular, $x_{1}, \ldots, x_{m}$ são linearmente independentes, de modo que formam uma base unitária de $X$. 
Por outro lado, para cada $j=1, \ldots, m$, seja $x_{j}^{*}: X \rightarrow \mathbb{R}$ dado por

$$
<x_{j}^{*}, z>=\frac{1}{a} \operatorname{det}\left[\hat{x}_{1}, \cdots, \hat{x}_{j-1}, \hat{z}, \hat{x}_{j+1} \cdots \hat{x}_{m}\right]
$$

Com isso, $x_{j}^{*} \in X^{*},<x_{j}^{*}, x_{i}>=\delta_{i, j}$ e $\left\|x_{j}^{*}\right\|_{X^{*}}=1$, pois, fixado $j \in\{1, \ldots, m\}$, para todo $z \in B$, temos $\left|f\left(x_{1}, \ldots, x_{j-1}, z, x_{j+1}, \ldots, x_{m}\right)\right| \leqslant a, \operatorname{logo}\left|<x_{j}^{*}, z>\right| \leqslant 1$ e o supremo é alcançado em $x_{j}:\left|<x_{j}^{*}, x_{j}>\right|=1$.

Que $x_{1}^{*}, \ldots, x_{m}^{*}$ formam uma base de $X^{*}$ é claro.

Agora, com a existência da base de Auerbach para espaços de dimensão finita, podemos prosseguir com um importante resultado sobre a distância de Banach-Mazur, que é uma melhoria (veja [7]) na estimativa feita por Mañé, segundo a qual todo espaço de Banach $X m$-dimensional sobre $\mathbb{R}$ satisfaz

$$
\mathrm{d}_{\mathrm{BM}}\left(X, \mathbb{R}_{\infty}^{m}\right) \leqslant \log \left(m 2^{m}\right)
$$

A propósito, definimos a distância de Banach-Mazur entre dois espaços normados isomorfos $X$ e $Y$ como sendo

$$
\mathrm{d}_{\mathrm{BM}}(X, Y)=\log \left(\inf \left\{\|T\|_{\mathcal{L}(X, Y)}\left\|T^{-1}\right\|_{\mathcal{L}(Y, X)}: T \in \mathcal{L}(X, Y), T^{-1} \in \mathcal{L}(Y, X)\right\}\right)
$$

Conforme o roteiro proposto, vamos provar a estimativa $\mathrm{d}_{\mathrm{BM}}\left(X, \mathbb{K}_{\infty}^{m}\right) \leqslant \log m$, usando a base de Auerbach de $X$ que obtivemos do lema anterior. Antes, porém, vale observar que

- $\mathrm{d}_{\mathrm{BM}}(X, Y) \geqslant 0$.

Dado um isomorfismo $T \in \mathcal{L}(X, Y)$, temos

$$
1=\left\|I_{Y}\right\|_{\mathcal{L}(Y)}=\left\|T T^{-1}\right\|_{\mathcal{L}(Y)} \leqslant\|T\|_{\mathcal{L}(X, Y)}\left\|T^{-1}\right\|_{\mathcal{L}(Y, X)}
$$

de modo que $\log 1 \leqslant \mathrm{~d}_{\mathrm{BM}}(X, Y)$.

- vale a desigualdade triangular para $\mathrm{d}_{\mathrm{BM}}$, ou seja,

$$
\mathrm{d}_{\mathrm{BM}}(X, Z) \leqslant \mathrm{d}_{\mathrm{BM}}(X, Y)+\mathrm{d}_{\mathrm{BM}}(Y, Z) .
$$


Dados isomorfismos $S: X \rightarrow Y$ e $T: Y \rightarrow Z$ (contínuos), determinamos um isomorfismo $T \circ S: X \rightarrow Z$ e temos

$$
\|(T \circ S)\|\left\|(T \circ S)^{-1}\right\| \leqslant\left(\|T\|\left\|T^{-1}\right\|\right)\left(\|S\|\left\|S^{-1}\right\|\right)
$$

de modo que

$$
\log \left(\|(T \circ S)\|\left\|(T \circ S)^{-1}\right\|\right) \leqslant \log \left(\|T\|\left\|T^{-1}\right\|\right)+\log \left(\|S\|\left\|S^{-1}\right\|\right),
$$

e isto basta.

- se $\operatorname{dim} X=\operatorname{dim} Y<\infty$, então $\mathrm{d}_{\mathrm{BM}}(X, Y)=0$ se, e somente se, $X$ e $Y$ são isometricamente isomorfos.

Claro que se existe um isomorfismo isométrico $T: X \rightarrow Y$, então $\mathrm{d}_{\mathrm{BM}}(X, Y)=0$. Por outro lado, note que $\mathcal{L}(X, Y)$ tem dimensão finita e que podemos definir $\mathrm{d}_{\mathrm{BM}}$ alternativamente como sendo

$$
\log \left(\inf \left\{\left\|T^{-1}\right\|_{\mathcal{L}(Y, X)}: T: X \rightarrow Y \text { é um isomorfismo com }\|T\|=1\right\}\right)
$$

Sejam $T_{n} \in \mathcal{L}(X, Y)$ tais que $\left\|T_{n}\right\|=1$ e $\left\|T_{n}^{-1}\right\| \rightarrow 1$. Uma vez que cada $T_{n}$ é um operador que pertence a bola fechada unitária de $\mathcal{L}(X, Y)$, a qual é compacta, extraímos uma subsequência $T_{n_{k}}$ tal que $\left\|T_{n_{k}}-T\right\| \rightarrow 0$ e $\|T\|=1$.

Mostremos que $T$ é injetora. Defina

$$
m(S):=\inf \left\{\|S x\|_{Y}:\|x\|_{X}=1\right\}
$$

e note que

i) $m(S)$ coincide com $\left(\left\|S^{-1}\right\|\right)^{-1}$ quando $S$ é invertível;

ii) $m(S)=0$ se, e somente se, $S$ é injetora;

iii) $\left|m\left(S_{1}\right)-m\left(S_{2}\right)\right| \leqslant\left\|S_{1}-S_{2}\right\|$.

Como $m\left(T_{n_{k}}\right)=\left\|T_{n_{k}}^{-1}\right\| \rightarrow 1$ e $\left\|T_{n_{k}}-T\right\| \rightarrow 0$, vemos que $m(T)=\lim _{k \rightarrow \infty} m\left(T_{n_{k}}\right)=1$; em particular, $T: X \rightarrow Y$ é injetora, logo um isomorfismo.

E pelo item $i$ ) acima, $1=m(T)=\left(\left\|T^{-1}\right\|\right)^{-1}$, implica que $\left\|T^{-1}\right\|=1$; ou seja, $T: X \rightarrow Y$ é um isomorfismo isométrico, como queríamos. 
Proposição 4.12. Seja $X$ um espaço de Banach $m$-dimensional sobre $\mathbb{R}$, então

$$
\mathrm{d}_{\mathrm{BM}}\left(X, \mathbb{R}_{\infty}^{m}\right) \leqslant \log m
$$

Demonstração: Seja $\left(\left(x_{1}, \cdots, x_{m}\right),\left(x_{1}^{*} \cdots, x_{m}^{*}\right)\right)$ uma base de Auerbach para $X$ e defina a aplicação

$$
\begin{aligned}
T: \quad \begin{array}{l}
\mathbb{R}_{\infty}^{m} \\
\lambda=\left(\lambda_{1}, \ldots, \lambda_{m}\right)
\end{array} & \longmapsto T \lambda:=\sum_{j=1}^{m} \lambda_{j} x_{j}, \\
& \longmapsto T
\end{aligned}
$$

daí, para cada $\lambda \in \mathbb{R}_{\infty}^{m}$,

$$
\|T \lambda\|_{X} \leqslant \sum_{j=1}^{m}\left|\lambda_{j}\right|\left\|x_{j}\right\|_{X}=\sum_{j=1}^{m}\left|\lambda_{j}\right| \leqslant m\|\lambda\|_{\infty}
$$

$\operatorname{logo}\|T\|_{\mathcal{L}\left(\mathbb{R}_{\infty}^{m}, X\right)} \leqslant m$. E por outro lado, dado $x=\sum_{j=1}^{m} \lambda_{j} x_{j} \in X$, temos que $T^{-1}(x)=$ $\left(\lambda_{1}, \ldots, \lambda_{m}\right) \in \mathbb{R}_{\infty}^{m}$ e então

$$
\left\|T^{-1} x\right\|_{\infty}=\max _{1 \leqslant j \leqslant m}\left|\lambda_{j}\right|=\max _{1 \leqslant j \leqslant m}\left|<x_{j}^{*}, x>\right| \leqslant\left(\max _{1 \leqslant j \leqslant m}\left\|x_{j}^{*}\right\|_{X^{*}}\right)\|x\|_{X}=\|x\|_{X},
$$

de onde obtemos que $\|T\|_{\mathcal{L}\left(\mathbb{R}_{\infty}^{m}, X\right)}\left\|T^{-1}\right\|_{\mathcal{L}\left(X, \mathbb{R}_{\infty}^{m}\right)} \leqslant m$, o que conclui a demonstração.

Da proposição segue que $d_{B M}(X, Y) \leqslant 2 \log m$, sempre que $X$ e $Y$ são espaços $m$ dimensionais.

Lema 4.13. Sejam $X$ um espaço de Banach sobre $\mathbb{R}$ e $Y$ um subespaço de dimensão $m$, então

$$
N\left(\rho, B_{Y}(0, r)\right) \leqslant\left(\frac{(m+1) r}{\rho}\right)^{m}
$$

sempre que $0<\rho \leqslant r$.

Além disso, as bolas na cobertura podem ser tomadas com centro em $Y$.

Demonstração: Considere o isomorfismo $T: \mathbb{R}_{\infty}^{m} \rightarrow Y$ da Proposição 4.12 tal que $\|T\|\left\|T^{-1}\right\| \leqslant m$ e note que

$$
B_{Y}(0, r)=T\left(T^{-1}\left(B_{Y}(0, r)\right)\right) \subset T\left(B_{\mathbb{R}_{\infty}^{m}}\left(0, r\left\|T^{-1}\right\|_{\mathcal{L}\left(Y, \mathbb{R}_{\infty}^{m}\right)}\right)\right)
$$


Tome $N$ o menor natural tal que

$$
N \frac{\rho}{\|T\|}>r\left\|T^{-1}\right\|
$$

de modo que o intervalo $\left(-r\left\|T^{-1}\right\|, r\left\|T^{-1}\right\|\right)$ pode ser coberto por $N$ intervalos de raio $\rho /\|T\|$ e, como $B_{\mathbb{R}_{\infty}^{m}}\left(0, r\left\|T^{-1}\right\|\right)$ é o cartesiano $\left(-r\left\|T^{-1}\right\|, r\left\|T^{-1}\right\|\right)^{m}$, vemos que ela pode ser coberta por $N^{m}$ bolas de raio $\rho /\|T\|$ centradas em pontos seus.

Com isso,

$$
N\left(\frac{\rho}{\|T\|}, B\left(0, r\left\|T^{-1}\right\|\right)\right) \leqslant N^{m} \leqslant\left(1+\frac{r\|T\|\left\|T^{-1}\right\|}{\rho}\right)^{m} \leqslant\left(\frac{r}{\rho}+m \frac{r}{\rho}\right)^{m} .
$$

Logo, por (4.3.1), podemos cobrir $B_{Y}(0, r)$ por este mesmo número de bolas de $Y$ de raio $\rho$ centradas em pontos de $Y$.

A estimativa de $N\left(\rho, B_{Y}(0, r)\right)$, no caso complexo, é por $(m+1)^{2 m}\left(\frac{\sqrt{2} r}{\rho}\right)^{2 m}$.

Agora vamos definir uma classe de aplicações que, a sua semelhança ao Teorema 4.9, admitem uma decomposição como soma de uma aplicação compacta e uma outra bem "controlada".

Dados $\lambda>0$ e espaços de Banach $X$ e $Z$, definimos

$$
\mathcal{L}_{\lambda}(X, Z)=\left\{T \in \mathcal{L}(X, Z): T=C+L \text { com } C \text { compacto e }\|L\|_{\mathcal{L}(X, Z)}<\lambda\right\}
$$

e usando $\mathcal{L}_{\lambda}(X)$ para abreviadamente denotar $\mathcal{L}_{\lambda}(X, X)$.

Como prometido, a classe $\mathcal{L}_{\lambda}(X, Z)$, a menos de um pequeno ajuste, é a que detém as propriedades necessárias para que a estimativa

$$
\operatorname{dist}_{H}\left(T\left(B_{X}(0,1)\right), T\left(B_{Y}(0,1)\right)\right)<\lambda
$$

seja verificada, para algum subespaço $Y$ de $X$.

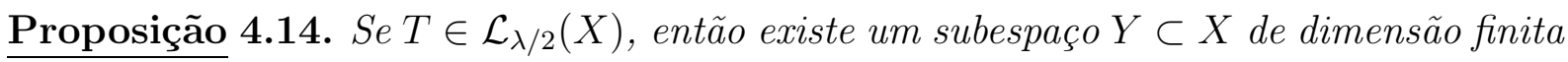
tal que

$$
T\left(B_{X}(0,1)\right) \subset \mathcal{O}_{\lambda}\left(T\left(B_{Y}(0,1)\right)\right)
$$

Demonstração: Escreva $T=C+L$, onde $C \in \mathcal{K}(X)$ e $L \in \mathcal{L}(X) \operatorname{com}\|L\|_{\mathcal{L}(X)}<\lambda / 2$. 
Afirmo que, para todo $\varepsilon>0$, existe um subespaço $Y^{\varepsilon} \subset X$ de dimensão finita tal que

$$
\operatorname{dist}_{\mathrm{H}}\left(C\left(B_{X}(0,1)\right), C\left(B_{Y^{\varepsilon}}(0,1)\right)\right)<\varepsilon
$$

Suponha que este não é o caso; isto é, suponha $\varepsilon_{0}>0$ tal que

$$
\operatorname{dist}_{\mathrm{H}}\left(C\left(B_{X}(0,1)\right), C\left(B_{Y}(0,1)\right)\right) \geqslant \varepsilon_{0}
$$

para todo subespaço $Y$ de $X$ com dimensão finita. Fixado $x_{1} \neq 0$ em $B_{X}(0,1)$, segue que

$$
\operatorname{dist}_{\mathrm{H}}\left(C\left(B_{X}(0,1)\right), C\left(B_{Y_{1}}(0,1)\right)\right) \geqslant \varepsilon_{0},
$$

em que $Y_{1}=\operatorname{span}\left\{x_{1}\right\}$.

Com isso, existe $x_{2} \neq 0$ em $B_{X}(0,1)$ tal que $d\left(C x_{2}, C\left(B_{Y_{1}}(0,1)\right)\right) \geqslant \varepsilon_{0} / 2$; em particular, $\left\|C x_{2}-C x_{1}\right\|_{X} \geqslant \varepsilon_{0} / 2$.

Aplicando o mesmo princípio para $Y_{2}=\operatorname{span}\left\{x_{1}, x_{2}\right\}$, obtemos da desigualdade

$$
\operatorname{dist}_{\mathrm{H}}\left(C\left(B_{X}(0,1)\right), C\left(B_{Y_{2}}(0,1)\right)\right) \geqslant \varepsilon_{0}
$$

um ponto $x_{3} \neq 0$ em $B_{X}(0,1)$ tal que $\left\|C x_{3}-C x_{1}\right\|_{X} \geqslant \varepsilon_{0} / 2$ e $\left\|C x_{3}-C x_{2}\right\|_{X} \geqslant \varepsilon_{0} / 2$.

Procedendo assim, construímos uma sequência $\left(x_{j}\right)_{j} \in B_{X}(0,1)$ não nula tal que

$$
\left\|C x_{j}-C x_{i}\right\|_{X} \geqslant \varepsilon_{0} / 2
$$

sempre que $i \neq j$, o que impede $\left(C x_{j}\right)_{j}$ de ter uma subsequência de Cauchy e viola a compacidade de $C$.

Seja $\mu$ tal que $2\|L\|_{\mathcal{L}(X)}<\mu<\lambda$ e tome $Y \subset X$ um subespaço de dimensão finita tal que

$$
\operatorname{dist}_{\mathrm{H}}\left(C\left(B_{X}(0,1)\right), C\left(B_{Y}(0,1)\right)\right)<\varepsilon:=\lambda-\mu .
$$

$\operatorname{Dados} x \in B_{X}(0,1)$ e $y \in B_{Y}(0,1)$, temos

$$
\begin{aligned}
\|T x-T y\|_{X} & \leqslant\|C x-C y\|_{X}+\|L x-L y\|_{X} \\
& <\|C x-C y\|_{X}+2\|L\| \\
& <\|C x-C y\|_{X}+\mu
\end{aligned}
$$


de modo que tomando o ínfimo em $B_{Y}(0,1)$ e então o supremo em $B_{X}(0,1)$, obtemos

$$
\operatorname{dist}_{H}\left(T\left(B_{X}(0,1)\right), T\left(B_{Y}(0,1)\right)\right) \leqslant \operatorname{dist}_{H}\left(C\left(B_{X}(0,1)\right), C\left(B_{Y}(0,1)\right)\right)+\mu<\lambda,
$$

como queríamos.

Agora, estamos em condições estabelecer requisitos sobre $T: X \rightarrow X$ e um compacto negativamente invariante $K$ de modo que

$$
\operatorname{dim}_{\mathrm{F}}(K) \leqslant-\frac{\log M}{\log \alpha}
$$

para constantes convenientes $M$ natural e $0<\alpha<1$.

Uma das condições é que $T$ seja diferenciável, no seguinte sentido: $T: U \subset X \rightarrow X$ é diferenciável (no sentido de Frechét) em $U$, quando existe $T^{\prime}(a) \in \mathcal{L}(X)$, para cada $a \in U$, de tal forma que

$$
\lim _{x \rightarrow a} \frac{\rho(x)}{\|x-a\|_{X}}=0
$$

em que $\rho(x):=T(x)-T(a)-T^{\prime}(a)(x-a)$.

Lema 4.15. Sejam $X$ um espaço de Banach real e $K \subset X$ um conjunto compacto. Suponha que

H1) $T: X \rightarrow X$ é uma aplicação continuamente diferenciável em uma vizinhança de $K$;

H2) $K$ é negativamente invariante: $K \subset T(K)$;

H3) existem $0<\alpha<1$ e $M$ natural tais que $N\left(\alpha, T^{\prime}(y) B_{X}(0,1)\right) \leqslant M$, para todo $y \in K$.

Então,

$$
\operatorname{dim}_{\mathrm{F}}(K) \leqslant-\frac{\log M}{\log \alpha}
$$

\section{Demonstração:}

Como $T$ é $C^{1}$ numa vizinhança de $K$, tem-se que $T$ é uniformemente diferenciável nesta vizinhança: pondo $\rho(x):=T(x)-T(y)-T^{\prime}(y)(x-y)$, dado $\eta>0$, existe $r_{0}=r_{0}(\eta)$ 
tal que, para todo $r \in\left(0, r_{0}\right]$,

$$
\begin{aligned}
x \in X, y \in K \text { tais que }\|x-y\|_{X}<r & \Rightarrow \frac{\|\rho(x)\|_{X}}{\|x-y\|_{X}}<\eta \\
& \Rightarrow \rho(x) \in B_{X}(0, r \eta) \\
& \Rightarrow T(x) \in T(y)+T^{\prime}(y) B_{X}(0, r)+B_{X}(0, r \eta) .
\end{aligned}
$$

Disso, para todos os $r \in\left(0, r_{0}\right]$ e $y \in K$, obtemos que

$$
T\left(B_{X}(y, r) \cap K\right) \subset T(y)+T^{\prime}(y) B_{X}(0, r)+B_{X}(0, r \eta)
$$

e graças a $H 3)$, podemos estimar

$$
N\left((\alpha+\eta) r, T\left(B_{X}(y, r) \cap K\right)\right) \leqslant M
$$

Agora, dado $\eta$ tal que $0<\eta<1-\alpha$, sejam $r_{0}=r_{0}(\eta)$ e $N_{0}:=N\left(r_{0}, K\right)$ :

$$
K=\bigcup_{i=1}^{N_{0}} B_{X}\left(y_{i}, r_{0}\right) \cap K
$$

para alguma escolha de pontos $y_{1}, \ldots, y_{N_{0}}$ em $K$.

Com isso,

$$
K \subset T(K) \subset \bigcup_{i=1}^{N_{0}} T\left(B_{X}\left(y_{i}, r_{0}\right) \cap K\right) \subset \bigcup_{i=1}^{N_{0}} \bigcup_{j=1}^{M} B_{X}\left(z_{i, j},(\alpha+\eta) r_{0}\right)
$$

de modo que $z_{i, j}$ são pontos de $K$ e $N\left((\alpha+\eta) r_{0}, K\right) \leqslant M N_{0}$. Mas como $\alpha+\eta<1$, temos $0<r=(\alpha+\eta) r_{0} \leqslant r_{0}$, e então por (4.3.4) e (4.3.5), segue que

$$
\begin{aligned}
K \subset T(K) & \subset \bigcup_{i=1}^{N_{0}} \bigcup_{j=1}^{M} T\left(B_{X}\left(z_{i, j},(\alpha+\eta) r_{0}\right) \cap K\right) \\
& \subset \bigcup_{i=1}^{N_{0}} \bigcup_{j=1}^{M} \bigcup_{l=1}^{M} B_{X}\left(z_{i, j, l},(\alpha+\eta)\left((\alpha+\eta) r_{0}\right)\right)
\end{aligned}
$$


daí $N\left((\alpha+\eta)^{2} r_{0}, K\right) \leqslant M^{2} N_{0}$, e procedendo assim, obtemos

$$
N\left((\alpha+\eta)^{m} r_{0}, K\right) \leqslant M^{m} N_{0}
$$

para todo natural $m$.

Da definição de $\operatorname{dim}_{\mathrm{F}}(K)$, concluimos que

$$
\operatorname{dim}_{\mathrm{F}}(K)=\limsup _{m \rightarrow \infty} \frac{\log N\left((\alpha+\eta)^{m} r_{0}, K\right)}{-\log \left((\alpha+\eta)^{m} r_{0}\right)} \leqslant \limsup _{m \rightarrow \infty} \frac{\log M^{m} N_{0}}{-\log \left((\alpha+\eta)^{m} r_{0}\right)}=-\frac{\log M}{\log (\alpha+\eta)}
$$

Uma vez que $0<\eta<1-\alpha$ é arbitrário, obtemos (4.3.3).

Os próximos dois teoremas intermedeiam o que fizemos até aqui com as aplicações a semigrupos. A fim de simplificar a notação do enunciado a seguir, definimos $\nu_{\lambda}(T)$ o menor natural $m$ para o qual existe um subespaço $Y \subset X$ com dimensão $m$ tal que

$$
\operatorname{dist}_{\mathrm{H}}\left(T\left(B_{X}(0,1)\right), T\left(B_{Y}(0,1)\right)\right)<\lambda
$$

com a convenção de que min $\varnothing=\infty$. Ou seja, $\nu_{\lambda}(T)$ é o menor natural $m$ para o qual existe um subespaço $Y \subset X$ com dimensão $m$ que satisfaz (4.3.2) da Proposição 4.14. Note que por tal resultado, se $T \in \mathcal{L}_{\lambda / 2}(X)$, então $\nu_{\lambda}(T)$ é finito.

Teorema 4.16. Sejam $\left(X,\|\cdot\|_{X}\right)$ um espaço de Banach real, $K \subset X$ um compacto $e$ $U \subset X$ um aberto tal que $U \supset K$.

Suponha $T: U \subset X \rightarrow X$ uma aplicação continuamente diferenciável em $U$ e uma constante $0<\lambda<1 / 2$ tal que $T^{\prime}(x) \in \mathcal{L}_{\lambda / 2}(X)$, para todo $x \in K$.

Então,

T1) $n_{\lambda}:=\sup _{x \in K} \nu_{\lambda}\left(T^{\prime}(x)\right)$ e $D_{0}:=\sup _{x \in K}\left\|T^{\prime}(x)\right\|_{\mathcal{L}(X)}$ são finitos;

T2) para todo $x \in K$, vale a estimativa

$$
N\left(2 \lambda, T^{\prime}(x) B_{X}(0,1)\right) \leqslant\left(\frac{\left(n_{\lambda}+1\right) D}{\lambda}\right)^{n_{\lambda}},
$$

em que $D$ denota o máximo entre $1 / 2$ e $D_{0}$. 
Além disso, se $K \subset T(K)$, então $\operatorname{dim}_{\mathrm{F}}(K)$ é finita. Mais precisamente,

$$
\operatorname{dim}_{F}(K) \leqslant-n_{\lambda} \frac{\log \left(\left(n_{\lambda}+1\right) D / \lambda\right)}{\log 2 \lambda}
$$

\section{Demonstração:}

Fixe $x \in K$.

Pela Proposição 4.14, existe um subespaço $Y_{x}$ de $X$ de dimensão finita tal que

$$
\operatorname{dist}_{\mathrm{H}}\left(T^{\prime}(x) B_{X}(0,1), T^{\prime}(x) B_{Y_{x}}(0,1)\right)<\mu<\lambda,
$$

em que $\mu=\mu(x)$.

Nestas condições, afirmo que existe $\delta=\delta(x)>0$ tal que $\nu_{\lambda}\left(T^{\prime}(y)\right) \leqslant \operatorname{dim} Y_{x}$, para todo $y \in B_{X}(x, \delta)$.

De fato, seja $\varepsilon=(\lambda-\mu) / 2>0$, daí existe $\delta>0$, que depende de $x$, tal que

$$
y \in U \operatorname{com}\|y-x\|_{X}<\delta \Rightarrow\left\|T^{\prime}(y)-T^{\prime}(x)\right\|_{\mathcal{L}(X)}<\varepsilon
$$

Nestas condições, dados $u \in B_{X}(0,1)$ e $v \in B_{Y_{x}}(0,1)$, vale

$\left\|T^{\prime}(y) u-T^{\prime}(y) v\right\|_{X} \leqslant\left\|T^{\prime}(y) u-T^{\prime}(x) u\right\|_{X}+\left\|T^{\prime}(x) u-T^{\prime}(x) v\right\|_{X}+\left\|T^{\prime}(x) v-T^{\prime}(y) v\right\|_{X}$,

e usando a estimativa por $\varepsilon$, depois tomando o ínfimo sobre $v$ e então o supremo sobre $u$, obtemos

$$
\begin{aligned}
\operatorname{dist}_{\mathrm{H}}\left(T^{\prime}(y) B_{X}(0,1), T^{\prime}(y) B_{Y_{x}}(0,1)\right) & \leqslant 2 \varepsilon+\operatorname{dist}_{\mathrm{H}}\left(T^{\prime}(x) B_{X}(0,1), T^{\prime}(x) B_{Y_{x}}(0,1)\right) \\
& <2 \varepsilon+\mu=\lambda
\end{aligned}
$$

como afirmado.

Da compacidade de $K$, existem $x_{1}, \ldots, x_{m} \in K$ tais que

$$
K \subset \bigcup_{i=1}^{m} B_{X}\left(x_{i}, \delta\left(x_{i}\right)\right) .
$$

Agora, pondo $p=\max _{1 \leqslant i \leqslant m} \operatorname{dim} Y_{x_{i}}$, o qual é finito, temos que $\nu_{\lambda}\left(T^{\prime}(x)\right) \leqslant p$, para todo 
$x \in K$, ou seja, $n_{\lambda}:=\sup _{x \in K} \nu_{\lambda}\left(T^{\prime}(x)\right)<\infty$.

Fixado $x \in K$, existe um subespaço $Y_{x}$ de $X \operatorname{com} \operatorname{dim} Y_{x} \leqslant n_{\lambda}$ tal que

$$
\operatorname{dist}_{\mathrm{H}}\left(T^{\prime}(x) B_{X}(0,1), T^{\prime}(x) B_{Y_{x}}(0,1)\right)<\lambda
$$

Uma vez que $T^{\prime}(x) Y_{x}$ é um subespaço de $X$ com dimensão no máximo $n_{\lambda}$, do Lema 4.13, sabemos que

$$
J:=N\left(\lambda, B_{T^{\prime}(x) Y_{x}}(0, D)\right) \leqslant\left(\frac{\left(n_{\lambda}+1\right) D}{\lambda}\right)^{n_{\lambda}}
$$

pois $0<\lambda<1 / 2 \leqslant D$.

Por (4.3.7), dado $u \in B_{X}(0,1)$, existe $v \in B_{Y_{x}}(0,1)$ tal que $\left\|T^{\prime}(x) u-T^{\prime}(x) v\right\|_{X}<\lambda$, em que

$$
T^{\prime}(x) v \in T^{\prime}(x) B_{Y_{x}}(0,1) \subset B_{T^{\prime}(x) Y_{x}}(0, D) \subset \bigcup_{j=1}^{J} B_{X}\left(x_{j}, \lambda\right),
$$

para alguma escolha de pontos $x_{1}, \ldots, x_{J}$ em $B_{T^{\prime}(x) Y_{x}}(0, D)$.

Com isso, $T^{\prime}(x) u \in B_{X}\left(x_{j}, 2 \lambda\right)$, para algum $j$, por desigualdade triangular. E então,

$$
T^{\prime}(x) B_{X}(0,1) \subset \bigcup_{j=1}^{J} B_{X}\left(x_{j}, 2 \lambda\right),
$$

de onde segue $T 2$ ).

Quanto à estimativa (4.3.6), é consequência do Lema 4.15,

As citadas estimativas, no caso em que $X$ é um espaço de Banach complexo, são

$$
N\left(2 \lambda, T^{\prime}(x) B_{X}(0,1)\right) \leqslant\left(\frac{\left(n_{\lambda}+1\right) D \sqrt{2}}{\lambda}\right)^{2 n_{\lambda}}
$$

e

$$
\operatorname{dim}_{\mathrm{F}}(K) \leqslant-2 n_{\lambda} \frac{\log \left(\left(n_{\lambda}+1\right) D \sqrt{2} / \lambda\right)}{\log 2 \lambda}
$$

respectivamente.

Teorema 4.17. Sejam $X$ um espaço de Banach real, $K \subset X$ um compacto e $U \subset X$ um 
aberto tal que $K \subset U$.

Suponha $T: U \rightarrow U$ uma aplicação continuamente diferenciável tal que $T(K)=K$ e uma constante $0<\varepsilon<1$ tal que $T^{\prime}(x) \in \mathcal{L}_{1-\varepsilon}(X)$, para todo $x \in K$, então

$$
\operatorname{dim}_{\mathrm{F}}(K)<\infty
$$

\section{Demonstração:}

Para cada $x \in K$, temos que $C_{x} \in \mathcal{K}(X)$ e $L_{x} \in \mathcal{L}(X)$ com $\left\|L_{x}\right\|_{\mathcal{L}(X)}<1-\varepsilon$ tais que

$$
T^{\prime}(x)=C_{x}+L_{x}
$$

Note que $T^{n}: U \rightarrow U$ está bem definida e é continuamente diferenciável em $U$, para todo $n$; daí pela Regra da Cadeia, obtemos

$$
\left(T^{n}\right)^{\prime}(x)=T^{\prime}\left(T^{n-1}(x)\right) \circ T^{\prime}\left(T^{n-2}(x)\right) \circ \ldots \circ T^{\prime}(T(x)) \circ T^{\prime}(x) .
$$

Uma vez que $x \in K$ implica $T(x), \ldots, T^{n-2}(x), T^{n-1}(x) \in K$, segue que, para cada $x \in K$ e cada $j=1, \ldots, n$,

$$
T^{\prime}\left(T^{n-j}(x)\right)=C_{j}+L_{j},
$$

em que $C_{j} \in \mathcal{K}(X)$ e $\left\|L_{j}\right\|_{\mathcal{L}(X)}<1-\varepsilon$, uma decomposição que depende de $x \in K$.

Assim, $\left(T^{n}\right)^{\prime}(x)=C+L$, sendo $C \in \mathcal{K}(X)$ (por ser soma de composições de operadores compactos e contínuos) e $L=L_{1} \circ L_{2} \circ \cdots \circ L_{n}$, com

$$
\|L\|_{\mathcal{L}(X)} \leqslant\left\|L_{1}\right\|\left\|L_{2}\right\| \cdots\left\|L_{n}\right\|<(1-\varepsilon)^{n}
$$

cota esta que independe de $x \in K$.

Dado $0<\lambda<1 / 2$, tome $n_{0} \in \mathbb{N}$ suficientemente grande tal que $(1-\varepsilon)^{n_{0}}<\lambda / 2$. Como a aplicação $S:=T^{n_{0}}: U \rightarrow U$ é $C^{1}(U), K \subset S(K)$ e $S^{\prime}(x) \in \mathcal{L}_{\lambda / 2}(X)$, para todo $x \in K$, segue do Teorema 4.16 que $\operatorname{dim}_{\mathrm{F}}(K)<\infty$.

Em resumo, garantimos a finitude da dimensão fractal de compactos $K$ invariantes por $T$ pedindo que $T^{\prime}(x) \in \mathcal{L}_{\lambda / 2}$, para cada $x \in K$. As estimativas de $\operatorname{dim}_{\mathrm{F}}(K)$, porém, carregam essa dependência do parâmetro $\lambda$. 
A seguir, verificamos que quando o posto de $T^{\prime}(x)$ é uniformemente limitado, digamos por $\nu$, a dimensão fractal do atrator do semigrupo associado a $T$ é dominada por $\nu$, de forma independente do parâmetro $\lambda$.

Corolário 4.18. Sejam $X$ um espaço de Banach real e $T \in \mathcal{C}^{1}(X)$.

Se o semigrupo $\left\{T^{n}: n \in \mathbb{Z}_{+}\right\}$possui um atrator global $\mathcal{A}$ e $\nu:=\sup _{x \in \mathcal{A}} \operatorname{rank}\left(T^{\prime}(x)\right)<\infty$, então

$$
\operatorname{dim}_{\mathrm{F}}(\mathcal{A}) \leqslant \nu
$$

\section{Demonstração:}

Uma vez que, dado $x \in \mathcal{A}$, a aplicação $T^{\prime}(x): X \rightarrow X$ é compacta, segue que $T^{\prime}(x) \in \mathcal{L}_{\lambda / 2}(X)$, qualquer que seja $\lambda>0$.

Assim, dado $0<\lambda<1 / 2$, do Teorema 4.16, segue que

$$
\operatorname{dim}_{\mathrm{F}}(\mathcal{A}) \leqslant-\nu \frac{\log ((\nu+1) D / \lambda)}{\log 2 \lambda}
$$

e tomando o limite quando $\lambda \rightarrow 0$, obtemos que $\operatorname{dim}_{\mathrm{F}}(\mathcal{A}) \leqslant \nu$.

Por fim, obtemos $\operatorname{dim}_{\mathrm{F}}(\mathcal{A})<\infty$ enfraquecendo as hipóteses ao pedir (novamente) que $T$ se decomponha como soma de um operador compacto $C$ e um contínuo "pequeno" $L$, e pedindo que o posto de $C^{\prime}(x)$ seja uniformemente limitado.

Corolário 4.19. Sejam $X$ um espaço de Banach real e $T: X \rightarrow X$ tal que $T=C+L$ e $C, L \in \mathcal{C}^{1}(X)$.

\section{Suponha que}

H1) o semigrupo discreto $\left\{T^{n}: n \in \mathbb{Z}_{+}\right\}$possui um atrator global $\mathcal{A}$;

H2) C tem posto finito em $\mathcal{A}$ :

$$
\nu:=\sup _{x \in \mathcal{A}} \operatorname{rank}\left(C^{\prime}(x)\right)<\infty
$$

H3) existe $\left(\lambda_{n}\right)_{n}$ positiva tal que $\lambda_{n} \rightarrow 0 e$

$$
\sup _{x \in \mathcal{A}}\left\|L^{\prime}\left(T^{n-1}(x)\right) \circ L^{\prime}\left(T^{n-2}(x)\right) \circ \cdots \circ L^{\prime}(x)\right\|_{\mathcal{L}(X)} \leqslant \lambda_{n} .
$$


Então,

$$
\operatorname{dim}_{\mathrm{F}}(\mathcal{A}) \leqslant \nu
$$

\section{Demonstração:}

Pelo teorema anterior, basta mostrar que $\operatorname{rank}\left(T^{\prime}(x)\right)$ é uniformemente limitado em $\mathcal{A}$.

Dado $x \in \mathcal{A}$, temos

$$
\begin{aligned}
\left(T^{n}\right)^{\prime}(x) & =T^{\prime}\left(T^{n-1}(x)\right) \circ T^{\prime}\left(T^{n-2}(x)\right) \circ \cdots \circ T^{\prime}(T(x)) \circ T^{\prime}(x) \\
& =\left(C^{\prime}\left(T^{n-1}(x)\right)+L^{\prime}\left(T^{n-1}(x)\right)\right) \circ \cdots \circ\left(C^{\prime}(T(x))+L^{\prime}(T(x))\right) \circ\left(C^{\prime}(x)+L^{\prime}(x)\right) \\
& =C_{n}+L^{\prime}\left(T^{n-1}(x)\right) \circ \cdots \circ L^{\prime}(T(x)) \circ L^{\prime}(x) \\
& =C_{n}+L_{n},
\end{aligned}
$$

de modo que $C_{n}$ é um operador compacto com posto no máximo $\nu$, e por $\left.H 3\right), L_{n}$ é um operador tal que $\left\|L_{n}\right\|_{\mathcal{L}(X)} \leqslant \lambda_{n}$. Em outras palavras, $\left(T^{n}\right)^{\prime}(x) \in \mathcal{L}_{\lambda_{n}}$.

Claramente, existe $Y_{n}$ subespaço de $X$ tal que $\operatorname{dim} Y_{n} \leqslant \nu$ e

$$
\operatorname{dist}_{\mathrm{H}}\left(\left(T^{n}\right)^{\prime}(x)\left(B_{X}(0,1)\right),\left(T^{n}\right)^{\prime}(x)\left(B_{Y_{n}}(0,1)\right)\right) \leqslant\left\|L^{\prime}\left(T^{n-1}(x)\right) \circ \cdots \circ L^{\prime}(x)\right\|_{\mathcal{L}(X)} \leqslant \lambda_{n} .
$$

Assim, dado $\lambda>0$, tome $n$ suficientemente grande de tal forma que $\left(T^{n}\right)^{\prime}(x) \in \mathcal{L}_{\lambda} \mathrm{e}$ $\nu_{\lambda}\left(\left(T^{n}\right)^{\prime}(x)\right) \leqslant \nu$. E uma vez que $T^{n}(\mathcal{A})=\mathcal{A}$, temos que

$$
\operatorname{dim}_{\mathrm{F}}(\mathcal{A}) \leqslant-\nu \frac{\log ((\nu+1) D / \lambda)}{\log 2 \lambda}
$$

para cada $0<\lambda<\frac{1}{2}$. Basta então fazer $\lambda \rightarrow 0$ para concluir que

$$
\operatorname{dim}_{\mathrm{F}}(\mathcal{A}) \leqslant \nu
$$

o que completa a prova.

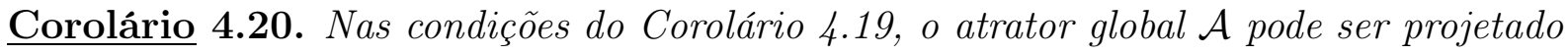
injetivamente em $\mathbb{R}^{N}$, para qualquer natural $N \geqslant 2 \nu+1$. 


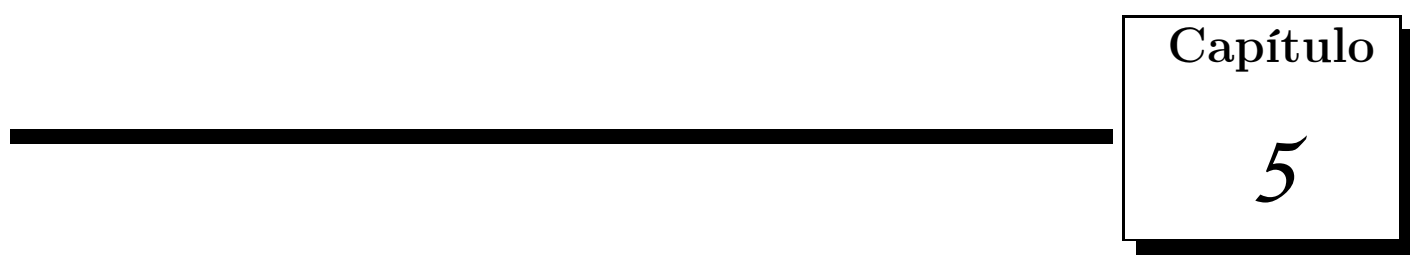

\section{Conclusões e Problemas em Aberto}

Já dizia Aristóteles,

"O começo de todas as ciências é o espanto de as coisas serem o que são."

Da curiosidade pelos fenômenos que nos cercam e da contagem de sacas produzidas e trocadas no início dos tempos, nasceu a ciência que hoje investiga a lógica sob os mais variados aspectos: a matemática. A íntima relação entre modelagem matemática, equações diferenciais e sistemas dinâmicos é uma herança que se mantém e que serve de alicerce para muito do que é proposto nas áreas econômica, física, química e biológica.

Entender o mundo por meio de modelos pressupõe entender tais modelos, que pressupõe entender a dinâmica do semigrupo associado a eles, ou seja, entender de que forma um ponto ou um conjunto evoluem com o tempo pela ação do semigrupo. Alguns problemas físicos e econômicos podem nos conduzir a uma modelagem em dimensão finita, situação para qual existe uma teoria clássica a nossa disposição. Porém, do ponto de vista matemático, somos frequentemente levados a problemas em dimensão infinita.

Como proceder então?

No início deste trabalho, definimos o que é um semigrupo e vimos que um objeto particular detém toda a informação da dinâmica assintótica no espaço de fase $X$ sob a ação deste semigrupo; é o chamado atrator global, que denotamos por $\mathcal{A}$ e que admite a caracterização

$$
\mathcal{A}=\bigcup_{B \in \mathcal{B}} \omega(B),
$$


sendo $\mathcal{B}$ a coleção dos subconjuntos limitados de $X$. É, de fato, o menor compacto invariante que atrai cada um dos subconjuntos limitados do espaço de fase pela ação do semigrupo.

Abordamos a Teoria das Dimensões em três etapas. De início, exploramos a mais largamente usada noção de dimensão em Topologia Geral, a dimensão topológica, reconhecendo quão difícil é checar que $\operatorname{dim}_{\mathrm{T}}\left(\mathbb{R}^{m}\right)=m$. A sua importância, porém, é devida ao Teorema da Imersão, um profundo resultado, tanto do ponto de vista topológico quanto do nosso ponto de vista. Como vimos, a essência do Teorema de Mañé advém do Teorema da Imersão, ambos como aplicações do Teorema da Categoria de Baire.

Em um segundo momento, consideramos a dimensão de Hausdorff que, além de ser convenientemente uma dimensão de caráter analítico em vez de topológico, detém importantes propriedades envolvendo funções Lipschitz e é a dimensão explorada por Mañé [24]. Por fim, a dimensão fractal facilita a aplicabilidade do Teorema de Mañé para semigrupos e, com isso, encerra o que buscamos compreender da Teoria das Dimensões.

Conhecendo a relação entre estas três dimensões em espaços métricos compactos,

$$
\operatorname{dim}_{\mathrm{T}}(X) \leqslant \operatorname{dim}_{\mathrm{H}}(X) \leqslant \operatorname{dim}_{\mathrm{F}}(X)
$$

a caracterização dos semigrupos que possuem atrator global e tendo o Teorema de Mañé à disposição, enunciamos o resultado que justifica a abordagem proposta.

Corolário 5.1. Seja $\{T(t): t \geqslant 0\}$ um semigrupo em um espaço de Banach $X$ com atrator global $\mathcal{A}$.

Se $\operatorname{dim}_{\mathrm{F}}(\mathcal{A})<\infty$, então $\mathcal{A}$ pode ser projetado injetivamente em $\mathbb{R}^{N}$, para $N$ suficientemente grande.

Com este resultado em mãos, o estudo dos atratores globais (e consequentemente, do comportamento assintótico do semigrupo) pode ser efetivamente reduzido a um estudo em dimensão finita, independente de o espaço de fase ser um espaço infinito dimensional.

Naturalmente nos perguntamos:

1) em que condições podemos garantir que o atrator global tem dimensão fractal finita?

2) quão abrangente é a classe dos semigrupos que satisfazem tal condição? 
Os semigrupos gradientes respondem satisfatoriamente a primeira pergunta, dadas as suas nobres propriedades dinâmicas, mas não a segunda, pois são admitidamente incomum na prática. Em [9], os autores justificam que os atratores exponenciais surgiram sob a proposta de considerar um compacto maior que o atrator global mas que detivesse ainda dimensão fractal finita e que mantivesse uma taxa de atração exponencial. A construção destes atratores exponenciais sai como um subproduto das hipóteses impostas; hipóteses estas que nos permitiram limitar a dimensão fractal do atrator global. Os semigrupos contemplados neste caso cobrem uma vasta classe dos semigrupos conhecidos. Por fim, tratamos também as estimativas para a dimensão fractal de compactos negativamente invariantes, segundo [23].

Vimos que, dado um conjunto compacto $K$ de um espaço de Banach $X$ com $\operatorname{dim}_{\mathrm{F}}(K)$ finita, existe uma projeção $P: X \rightarrow \mathbb{R}^{N}$, para $N>2 \operatorname{dim}_{\mathrm{F}}(K)+1$, de modo que

$$
\left.P\right|_{K}: K \subset X \rightarrow \mathbb{R}^{N}
$$

é uma aplicação injetora. Mais do que isso, a coleção de tais projeções forma um conjunto denso no espaço das projeções $\mathcal{P}\left(X, \mathbb{R}^{N}\right)$.

Em [6] e [27], vemos que a inversa $\left(\left.P\right|_{K}\right)^{-1}: P\left(\mathbb{R}^{N}\right) \rightarrow K$ é uma aplicação Hölder contínua com expoente $\theta$, o qual é dependente da dimensão $N$ da seguinte forma

$$
0<\theta<\frac{N-2 \operatorname{dim}_{\mathrm{F}}(K)}{N(1+\tau)}
$$

em que $\tau$ denota a dual thickness de $X$; quando $\tau=0$, o expoente de Hölder $\theta$ pode ser tomado arbitrariamente próximo de 1 , basta considerar $N$ suficientemente grande.

Além disso, fazendo $N \rightarrow \infty$, o expoente de Hölder limite é dado por $\frac{1}{1+\tau}$, o qual é o ótimo, em termos de thickness.

Um outro ponto a ser investigado é o quanto a exigência de $P: X \rightarrow \mathbb{R}^{N}$ ser linear restringe a regularidade que a sua inversa pode alcançar. Em particular, podemos nos perguntar se é possível encontrar, em geral, uma imersão (não necessariamente linear) de $K \mathrm{em} \mathbb{R}^{N}$ que seja uma aplicação bi-Lipschitz. A finitude da dimensão de Assouad, uma generalização da dimensão fractal, emerge como uma condição necessária, mas não suficiente. 
Denotando por $\operatorname{dim}_{\mathrm{A}}(K)$ a dimensão de Assouad de $K$, obtemos o seguinte encadeamento de desigualdades:

$$
\operatorname{dim}_{\mathrm{T}}(K) \leqslant \operatorname{dim}_{\mathrm{H}}(K) \leqslant \operatorname{dim}_{\mathrm{F}}(K) \leqslant \operatorname{dim}_{\mathrm{A}}(K)
$$

O Lema 9.9 em [27] fornece um exemplo de um compacto enumerável $K_{\alpha}$ em um espaço de Hilbert $H$ tal que

$$
0=\operatorname{dim}_{\mathrm{T}}\left(K_{\alpha}\right)=\operatorname{dim}_{\mathrm{H}}\left(K_{\alpha}\right) \leqslant \operatorname{dim}_{\mathrm{F}}\left(K_{\alpha}\right)=1 / \alpha \leqslant \operatorname{dim}_{\mathrm{A}}\left(K_{\alpha}\right)=\infty
$$

de modo que $K_{\alpha}$ não admite uma imersão bi-Lipschitz sobre um espaço finito dimensional.

O interesse por resultados de imersão está atrelado à questão de ser ou não possível reproduzir a dinâmica de um atrator global finito dimensional por meio de um apropriado sistema finito dimensional de equações diferenciais ordinárias. Noutras palavras, levanta-se a seguinte pergunta:

"Se for possível estudar a dinâmica do atrator em dimensão finita, será também possível encontrar um sistema em algum $\mathbb{R}^{m}$ segundo o qual a dinâmica representa a dinâmica do semigrupo original?"

Por ora, dispomos apenas de resultados parciais neste sentido, consulte [27] para referências. 
Apêndice

$\mathcal{A}$

\section{Espaços de Dimensão Topológica Nula}

Este apêndice reúne resultados sobre espaços métricos separáveis que têm dimensão topológica nula, com o intuito de complementar a Seção 2.1, bem como torná-la mais sucinta em seu objetivo de mostrar que $\mathbb{R}^{m}$ tem dimensão topológica $m$. A referência básica novamente é o texto de Jan Van Mill [21].

Fixe $X$ um espaço métrico separável não vazio. Também vamos trabalhar apenas com subespaços $Y$ de $X$ não vazios.

Relembre que, pela Definição 2.7,

$$
\operatorname{dim}_{\mathrm{T}}(X)=0
$$

se, e somente se, dados $F, G \subset X$ fechados disjuntos, existe uma $(F, G)$-partição $C$ tal que $C=\varnothing$, se, e somente se, dados $F, G \subset X$ fechados disjuntos, existem abertos disjuntos $U, V \subset X$ tais que

$$
F \subset U, G \subset V \text { e } X \backslash \varnothing=U \cup V
$$

se, e somente se, dados $F, G \subset X$ fechados disjuntos, $X$ é a reunião de dois conjuntos abertos $U$ e $V$ (logo, também fechados) disjuntos tais que

$$
F \subset U \text { e } G \subset V
$$

se, e somente se, dados $F, G \subset X$ fechados disjuntos, existe um aberto fechado $U$ que contém $F$ e não intercepta $G$. 
Acontece que os espaços $X$ de dimensão topológica nula são, em um certo sentido, os blocos com os quais se constrói os outros espaços (consulte o Apêndice B), o que até justifica seu estudo ser feito à parte com mais detalhes.

Proposição A.1. São equivalentes:

a) $\operatorname{dim}_{\mathrm{T}}(X)=0$;

b) para cada $x \in X$ e cada vizinhança $U$ de $x$, existe um conjunto $B \subset X$ aberto fechado tal que $x \in B \subset U$;

c) os conjuntos abertos fechados de $X$ formam uma base (aberta) de $X$;

d) X tem uma base enumerável formada por abertos fechados;

e) toda cobertura aberta de $X$ admite um refinamento formado por abertos dois a dois disjuntos (consequentemente, abertos fechados).

\section{Demonstração:}

A implicação $a) \Rightarrow b$ ) é consequência imediata da observação feita anteriormente; enquanto que $b) \Rightarrow c$ ) é trivial.

$c) \Rightarrow d)$

Seja $\mathcal{B}=\left\{B_{j}: j \in \mathbb{N}\right\}$ uma base enumerável de $X$ formada por abertos. Por $c$ ), para cada $j$, existe $\mathcal{U}_{j}$ uma família de abertos fechados de $X$ tais que $B_{j}$ é escrito como reunião deles.

Uma vez que $B_{j}$ é um espaço de Lindelöf (pois ser métrico separável), podemos já assumir que $\mathcal{U}_{j}$ é enumerável, e assim, $\mathcal{U}=\bigcup_{j=1}^{\infty} \mathcal{U}_{j}$ é uma base enumerável de $X$ formada por abertos fechados de $X$.

$$
d) \Rightarrow e)
$$

Sejam $\mathcal{B}=\left\{B_{j}: j \in \mathbb{N}\right\}$ uma base enumerável de $X$ formada por abertos fechados e $\mathcal{U}$ uma cobertura aberta de $X$.

Dado $x \in X$, existe um $j(x) \in \mathbb{N}$ tal que $B_{j(x)} \subset U$, para algum $U \in \mathcal{U}$. Sendo assim, a coleção $\left\{B_{j(x)}: x \in X\right\} \subset \mathcal{B}$ é uma cobertura enumerável de $X$ e um refinamento de $\mathcal{U}$; e podemos denotá-la por $\left\{B_{n}: n \in \mathbb{N}\right\}$. 
Para cada $n$ natural, defina $V_{n} \subset X$ por

$$
V_{n}=B_{n} \backslash \bigcup_{k=1}^{n} B_{k}
$$

de modo que $\mathcal{V}=\left\{V_{n}: n \in \mathbb{N}\right\}$ consiste de conjuntos abertos dois a dois disjuntos, em que cada um deles está contido nalgum aberto de $\mathcal{U}$.

É fácil verificar que $\mathcal{V}$ é uma cobertura de $X$ : de fato, dado $x \in X$, seja $n(x)$ o menor natural $n$ tal que $x \in B_{n}$. Logo, $x \in V_{n}$.

$e) \Rightarrow a)$

Sejam $F, G \subset X$ fechados disjuntos.

Para cada $x \in X$, existe uma vizinhança $U_{x}$ de $x$ tal que $U_{x} \cap F=\varnothing$ ou $U_{x} \cap G=\varnothing$. Por $e$ ), a cobertura aberta $\mathcal{U}=\left\{U_{x}: x \in X\right\}$ de $X$ tem refinamento aberto $\mathcal{V}$ formados por conjuntos dois a dois disjuntos.

Agora, seja $U$ a reunião de todos os $V \in \mathcal{V}$ tais que $V \cap F \neq \varnothing$. Com isso,

i) $U$ é um aberto fechado, uma vez que cada $V \in \mathcal{V}$ é um aberto fechado de $X$, e

ii) $U$ contém $F$ e não intercepta $G$.

O Teorema A.6 sumariza importantes informações sobre espaços de dimensão topológica nula, o qual é invocado na Seção 2.1. Sua demonstração, porém, exige uma reflexão sobre como podemos separar abertos disjuntos em $X$. Para tanto, consideraremos três lemas: A.2, A.4 e A.5.

Denote por $\mathcal{F}_{X}$ a coleção dos conjuntos fechados de $X$ e considere a convenção $d(x, \varnothing)=$ $\infty$, para todo $x \in X$.

Lema A.2. Sejam $Y$ um subespaço de $X$ e $\kappa: \mathcal{F}_{Y} \rightarrow \mathcal{F}_{X}$ definida por

$$
\kappa(F)=\{x \in X: d(x, F) \leqslant d(x, Y \backslash F)\}
$$

então $\kappa$ tem as seguintes propriedades:

1) $\kappa(\varnothing)=\varnothing$ e $\kappa(Y)=X$; 
2) $\kappa(F) \cap Y=F$, para todo $F \in \mathcal{F}_{Y}$;

3) se $F, G \in \mathcal{F}_{Y}$ e $F \subset G$, então $\kappa(F) \subset \kappa(G)$, e

4) se $F, G \in \mathcal{F}_{Y}$, então $\kappa(F \cup G)=\kappa(F) \cup \kappa(G)$.

\section{Demonstração:}

Não é difícil verificar que $\kappa$ está bem definida e que valem os ítens 1), 2) e 3).

Sejam $F, G \in \mathcal{F}_{Y}$. Por 3), vemos que

$$
\kappa(F) \cup \kappa(G) \subset \kappa(F \cup G) .
$$

Dado $x \in \kappa(F \cup G)$, como $d(x, F \cup G) \in\{d(x, F), d(x, G)\}$, podemos assumir sem perda de generalidade que $d(x, F \cup G)=d(x, F)$, daí

$$
d(x, F)=d(x, F \cup G) \leqslant d(x, Y \backslash(F \cup G))
$$

Afirmo que $x \in \kappa(F)$. Uma vez que $d(x, F)=d(x, F \cup G) \leqslant d(x, G)$, segue que

$$
d(x, F) \leqslant d(x,(Y \backslash(F \cup G)) \cup G) \leqslant d(x, Y \backslash F),
$$

pois $Y \backslash F \subset(Y \backslash(F \cup G)) \cup G$.

Definição A.3. Subconjuntos $F, G \subset X$ são ditos separados se

$$
\bar{F} \cap G=\varnothing \quad \text { e } F \cap \bar{G}=\varnothing .
$$

Note que se $F$ e $G$ são disjuntos e são ou ambos abertos ou ambos fechados, então $A$ e $B$ são separados.

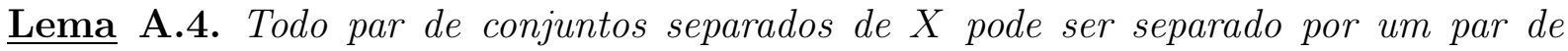
abertos disjuntos.

\section{Demonstração:}

Sejam $F, G \subset X$ subconjuntos separados e ponha $Y=F \cup G$ no Lema A.2, Claro que $F, G \in \mathcal{F}_{F \cup G}$, logo existem $F^{\prime}, G^{\prime} \subset X$ fechados de $X$ tais que $F \subset F^{\prime}, G \subset G^{\prime}$ e $F^{\prime} \cup G^{\prime}=X$. 
Assim, $U=X \backslash G^{\prime}$ e $V=X \backslash F^{\prime}$ são vizinhanças de $F$ e $G$, respectivamente, e além disso,

$$
U \cap V=\varnothing .
$$

O próximo resultado é simples mas fundamental para a Teoria das Dimensões. Diz que se tivermos uma partição de certos fechados relativos a um subespaço $Y$, então esta partição é a "restrição" de uma partição em $X$.

Lema A.5. Sejam $Y$ um subespaço de $X$ e $F, G \subset X$ fechados disjuntos.

Suponha que

i) existem $U$ e $V$ vizinhanças de $F$ e $G$, respectivamente, tais que $\bar{U} \cap \bar{V}=\varnothing$;

ii) S é uma $(\bar{U} \cap Y, \bar{V} \cap Y)$-partição em $Y$.

Então, existe uma $(F, G)$-partição $T$ em $X$ tal que $T \cap Y \subset S$.

\section{Demonstração:}

Escreva $Y \backslash S$ como a reunião disjunta de dois abertos em $Y$, digamos $A$ e $B$, tais que

$$
\bar{U} \cap Y \subset A \text { e } \bar{V} \cap Y \subset B .
$$

Uma vez que $A \cap V=\varnothing$, obtemos $\bar{A} \cap G=\varnothing$; e similarmente $\bar{B} \cap F=\varnothing$. Disto, vemos que os conjuntos $F \cup A$ e $G \cup B$ são separados e então, pelo Lema A.4, $F \cup A$ e $G \cup B$ possuem vizinhanças disjuntas $U^{\prime}$ e $V^{\prime}$, respectivamente.

Agora, basta considerar $T=X \backslash\left(U^{\prime} \cup V^{\prime}\right)$ :

$$
T \cap Y=\left(Y \backslash U^{\prime}\right) \cap\left(Y \backslash V^{\prime}\right) \subset(Y \backslash A) \cap(Y \backslash B)=S .
$$

Teorema A.6. Seja $Y$ um subespaço de $X$. Valem as seguintes afirmações:

1) se $\operatorname{dim}_{\mathrm{T}}(X)=0$, então $\operatorname{dim}_{\mathrm{T}}(Y)=0$;

2) se $\operatorname{dim}_{\mathrm{T}}\left(X_{n}\right)=0$, para todo $n$ natural, então $\operatorname{dim}_{\mathrm{T}}\left(\prod_{n=1}^{\infty} X_{n}\right)=0$; 
3) se $\operatorname{dim}_{\mathrm{T}}(X)=0$, então $X$ pode ser imerso em $\{0,1\}^{\infty}$;

4) se $\operatorname{dim}_{\mathrm{T}}(X)=0$, então $X$ admite uma compactificação de dimensão topológica nula;

5) se $\operatorname{dim}_{\mathrm{T}}(Y)=0$, então, dados $F, G \subset X$ fechados disjuntos, existe uma $(F, G)$ partição $T$ em $X$ tal que $T \cap Y=\varnothing$;

6) se $X$ pode ser escrito como uma reunião de até $m+1$ subespaços de dimensão topológica nula, então

$$
\operatorname{dim}_{\mathrm{T}}(X) \leqslant m
$$

\section{Demonstração:}

Vale 1)

Segue da Proposição A.1, item c).

Vale 2)

Para cada $n$, seja $\mathcal{B}_{n}$ uma base de $X_{n}$ formada por conjuntos abertos fechados, e note que a família dos conjuntos da forma

$$
E_{1} \times \cdots \times E_{k} \times X_{k+1} \times X_{k+2} \times \cdots,
$$

em que $E_{1} \in \mathcal{B}_{1}, \ldots, E_{k} \in \mathcal{B}_{k}$, é uma base enumerável de $\prod_{n=1}^{\infty} X_{n}$ formada por abertos fechados. Pela Proposição A.1, item $d$ ), vemos que

$$
\operatorname{dim}_{\mathrm{T}}\left(\prod_{n=1}^{\infty} X_{n}\right)=0
$$

Vale 3)

Seja $\mathcal{B}=\left\{B_{n}: n \in \mathbb{N}\right\}$ uma base enumerável de $X$ formada por abertos fechados de $X$ (pela Proposição A.1, item $d)$ ). Para cada $n$ natural, defina $f_{n}: X \rightarrow\{0,1\}$ por

$$
f_{n}(x)= \begin{cases}0, & \text { se } x \in B_{n} \\ 1, & \text { se } x \notin B_{n}\end{cases}
$$


de modo que $f_{n}$ é contínua. Não é difícil conferir que $F: X \rightarrow\{0,1\}^{\infty}$ definido por

$$
F(x)=\left(f_{n}(x)\right)_{n}
$$

é uma imersão.

Vale 4)

Por 3), podemos assumir que $X$ é um subespaço de $K=\{0,1\}^{\infty}$, o qual é compacto. Como $\operatorname{dim}_{\mathrm{T}}(K)=0$, por 2), basta tomar a desejada compactificação de $X$ como sendo o fecho de $X$ em $K$.

Vale 5)

Dados $F, G \subset X$ fechados disjuntos, tome $U$ e $V$ vizinhanças de $F$ e $G$, respectivamente, tais que $\bar{U} \cap \bar{V}=\varnothing$, pelo Corolário 1.8.

Como $\operatorname{dim}_{\mathrm{T}}(Y)=0$, sabemos que $\varnothing$ é uma $(\bar{U} \cap Y, \bar{V} \cap Y)$-partição em $Y$. Agora, basta aplicar o Lema A.5 para obter uma $(F, G)$-partição $T$ em $X$ tal que $T \cap Y=\varnothing$.

Vale 6)

Suponha $X_{1}, \ldots, X_{m+1} \subset X$ tais que

$$
X=\bigcup_{j=1}^{m+1} X_{j}
$$

e $\operatorname{dim}_{\mathrm{T}}\left(X_{j}\right) \leqslant-1$, pois alguns podem eventualmente ser vazios.

Dada uma família $\left\{\left(F_{j}, G_{j}\right): j=1, \ldots, m+1\right\}$ de $m+1$ pares de fechados disjuntos, sabemos por 5$)$ que, para cada $j=1, \ldots, m+1$, existe uma $\left(F_{j}, G_{j}\right)$-partição $T_{j}$ em $X$ tal que $T_{j} \cap X_{j}=\varnothing$.

Com isso,

$$
\bigcap_{j=1}^{m+1} T_{j}=\varnothing,
$$

de modo que $\left\{\left(F_{j}, G_{j}\right): j=1, \ldots, m+1\right\}$ é não essencial, $\operatorname{logog}_{\operatorname{dim}}(X) \leqslant m$.

O item 6) do Teorema A.6 é de fundamental importância para a Teoria da Dimensão, ainda mais que a recíproca é verdadeira; uma consequência do Teorema da Imersão para Espaços Métricos Separáveis (Teorema 3.3). 


\section{Corolário A.7. (Jan van Mill [21], Corollary 4.4.8)}

São equivalentes:

a) $\operatorname{dim}_{\mathrm{T}}(X) \leqslant m ; e$

b) $X$ pode ser escrito como uma reunião de até $m+1$ subespaços de dimensão topológica nula.

Do Lema A.5, obtemos uma expressiva consequência, que será necessária no Apêndice B para garantir que as dimensões topológica e pequena indutiva coincidem.

\section{Corolário A.8. (Jan van Mill [21], Corollary 4.1.8)}

Se $\operatorname{dim}_{\mathrm{T}}(X) \geqslant m$, então existem $F$ e $G$ fechados disjuntos tais que

$$
\operatorname{dim}_{\mathrm{T}}(L) \geqslant m-1
$$

para toda $(F, G)$-partição L.

Isto encerra nosso estudo sobre os espaços de dimensão topológica nula, mas muito mais pode ser dito.

\section{Proposição A.9. (Jan van Mill [21], Proposition 4.2.3)}

Um subespaço $Y$ de $\mathbb{R}$ não contém intervalos se, e somente se, $\operatorname{dim}_{\mathrm{T}}(Y)=0$.

Da Proposição A.9, vemos que

$$
\operatorname{dim}_{\mathrm{T}}(C)=0,
$$

em que $C$ denote o conjunto de Cantor dos terços médios. De fato, é possível mostrar que $C$ é (topologicamente) o único espaço métrico separável compacto (não vazio) sem pontos isolados que tem dimensão topológica nula. Além disso, todo espaço (métrico separável) de dimensão topológica nula pode ser imerso em $C$.

Para mais informações, consulte Jan Van Mill [21]. 


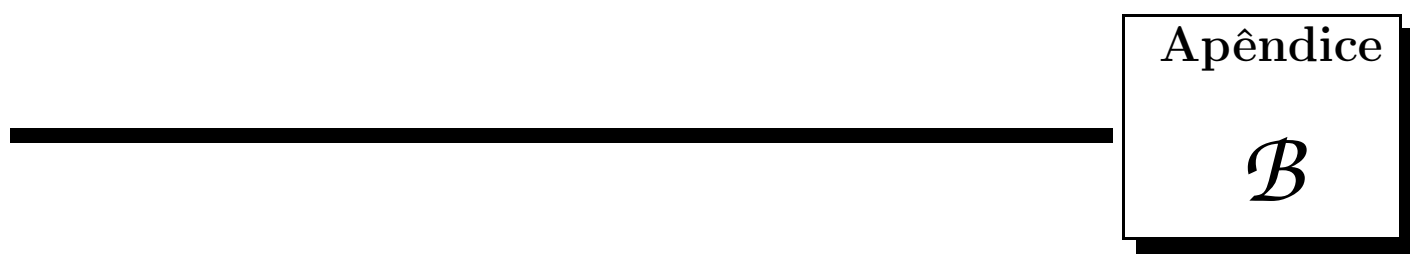

\section{Dimensões Indutivas}

Fixemos $X=(X, \mathrm{~d})$ um espaço métrico separável.

Deixando de lado a dimensão de Hausdorff, podemos associar a $X$, três "distintas" dimensões:

1) a dimensão topológica, $\operatorname{dim}_{\mathrm{T}}(X)$, a qual foi discutida na Seção 2.1 e preserva a intuição que temos sobre dimensão;

2) ind $(X)$, a pequena dimensão indutiva, que introduzimos na Seção 2.4:

3) Ind $(X)$, a grande dimensão indutiva, a qual será definida a seguir.

Como prometido na Seção 2.1, estas três são de fato iguais. As dimensões ind e Ind são importantes pois em certas situações é mais fácil lidar com elas em vez de com $\operatorname{dim}_{\mathrm{T}}$, como quando se tem que mostrar que

$$
\operatorname{ind}(X \times Y) \leqslant \operatorname{ind}(X)+\operatorname{ind}(Y),
$$

para dois espaços métricos separáveis quaisquer, de modo que a $\operatorname{dim}_{\mathrm{T}}$ satisfaz a mesma relação; no entanto, verificar isto diretamente para $\operatorname{dim}_{\mathrm{T}}$ é uma tarefa desagradável. Consulte Jan van Mill [21].

De fato, a referência principal é Jan van Mill [21], mas há muito conteúdo em comum com Engelking [15], Hurewicz \& Wallman [20] e Edgar [12].

Para organizar as ideias, definimos as três noções desde já. 
Definição B.1. Definimos a dimensão topológica de $X, \operatorname{com}$ a notação $\operatorname{dim}_{\mathrm{T}}(X)$, como sendo

1) $\operatorname{dim}_{\mathrm{T}}(X)=-1$, se $X$ é vazio;

2) $X$ não vazio tem dimensão topológica finita menor ou igual a $m \in \mathbb{Z}_{+}$, indicando por $\operatorname{dim}_{\mathrm{T}}(X) \leqslant m$, quando toda família de $m+1$ pares de subconjuntos fechados disjuntos de $X$ for não essencial;

3) X tem dimensão topológica $m$, indicando por $\operatorname{dim}_{\mathrm{T}}(X)=m$, se $m$ é o menor inteiro positivo tal que $\operatorname{dim}_{\mathrm{T}}(X) \leqslant m$;

4) $X$ tem dimensão topológica infinita, e escrevemos $\operatorname{dim}_{\mathrm{T}}(X)=\infty$, se não existe $m$ tal que $\operatorname{dim}_{\mathrm{T}}(X) \leqslant m$.

Tenha sempre em mente o Teorema 2.17, o qual fornece uma lista de definições equivalentes para a dimensão topológica.

Definição B.2. Definimos a pequena dimensão indutiva de $X$, com a notação ind $(X)$, como sendo

1) $\operatorname{ind}(X)=-1$, se $X$ é vazio;

2) $X$ não vazio tem pequena dimensão indutiva finita menor ou igual a $m \in \mathbb{Z}_{+}$, indicando por $\operatorname{ind}(X) \leqslant m$, quando, dados $x \in X$ e $F \subset X$ um fechado disjunto de $\{x\}$, existe uma $(\{x\}, F)$-partição $C$ tal que ind $(C) \leqslant m-1$;

3) $X$ tem pequena dimensão indutiva igual a $m$, indicando por $\operatorname{ind}(X)=m$, se $m$ é o menor inteiro positivo tal que ind $(X) \leqslant m$;

4) $X$ tem pequena dimensão indutiva infinita, e escrevemos ind $(X)=\infty$, se não existe $m$ tal que $\operatorname{ind}(X) \leqslant m$.

É fácil verificar que as Definições 2.42 e B.2 de pequena dimensão indutiva são equivalentes. Em todo caso, a argumentação está disponível para conferência na Proposição 1.1.4 em Engelking [15]. 
Definição B.3. Definimos a grande dimensão indutiva de $X$, com a notação $\operatorname{Ind}(X)$, como sendo

1) $\operatorname{Ind}(X)=-1$, se $X$ é vazio;

2) $X$ não vazio tem grande dimensão indutiva finita menor ou igual a $m \in \mathbb{Z}_{+}$, indicando por $\operatorname{Ind}(X) \leqslant m$, quando, dados $F, G \subset X$ fechados disjuntos, existe uma $(F, G)$-partição $C$ tal que $\operatorname{Ind}(C) \leqslant m-1$;

3) $X$ tem grande dimensão indutiva igual a $m$, indicando por $\operatorname{Ind}(X)=m$, se $m$ é o menor inteiro positivo tal que $\operatorname{Ind}(X) \leqslant m$;

4) $X$ tem grande dimensão indutiva infinita, e escrevemos $\operatorname{Ind}(X)=\infty$, se não existe $m$ tal que $\operatorname{Ind}(X) \leqslant m$.

Que fique claro, porém, que a grande dimensão indutiva só será utilizada nos últimos resultados do apêndice. O tratamento proposto em Jan Van Mill [21] nos induz a ressaltar a maior aplicabilidade da pequena dimensão indutiva; o que justifica termos nos referido a ela simplesmente como dimensão indutiva na Seção 2.4 .

Proposição B.4. Vale

$$
\operatorname{dim}_{\mathrm{T}}(X)=0 \Leftrightarrow \operatorname{ind}(X)=0
$$

\section{Demonstração:}

Da Definição B.2, vemos que ind $(X)=0$ se, e somente se, a coleção dos abertos fechados de $X$ formam um base. Da Proposição A.1, isto equivale a $\operatorname{dim}_{\mathrm{T}}(X)=0$.

Vamos trabalhar com algumas propriedades da pequena dimensão indutiva. É útil ter em mente (pois será diversas vezes usado) que, se $Y$ é um subespaço de $X, y \in Y$ e $F \subset Y$ é um fechado de $Y$ que não contém $y$, então $y \notin \bar{F}^{X}$, i.e., $y$ não pertence ao fecho de $F$ em $X$.

Lema B.5. Se $Y$ é subespaço de $X$, então ind $(Y) \leqslant \operatorname{ind}(X)$.

\section{Demonstração:}

Claro que vale se ind $(X)=-1$. 
Por indução, assuma que o lema seja verdadeiro para todos os espaços $Z$ tais que $\operatorname{ind}(Z) \leqslant m-1$, com $m \in \mathbb{Z}_{+}$, e assuma que ind $(X) \leqslant m$.

Sejam $x \in Y$ e $F \cap Y$ um fechado de $Y$ que não contém $x$, com $F \subset X$ fechado.

Uma vez que ind $(X) \leqslant m$, existe uma $(\{x\}, F)$-partição $L$ em $X$ tal que ind $(L) \leqslant$ $m-1$, daí $L \cap Y$ é uma $(\{x\}, F \cap Y)$-partição em $Y$. Agora, como $L \cap Y$ é subespaço de $L$ e $\operatorname{ind}(L) \leqslant m-1$, segue da hipótese de indução, que ind $(L \cap Y) \leqslant m-1$.

Com isso, mostramos que ind $(Y) \leqslant m$, o que completa a demonstração.

Teorema B.6. Se $Y_{1}$ e $Y_{2}$ são subespaços de $X$, então

$$
\operatorname{ind}\left(Y_{1} \cup Y_{2}\right) \leqslant \operatorname{ind}\left(Y_{1}\right)+\operatorname{ind}\left(Y_{2}\right)+1
$$

\section{Demonstração:}

Podemos assumir que ind $\left(Y_{1}\right)$ e ind $\left(Y_{2}\right)$ são finitas, caso contrário não há o que provar.

Se ind $\left(Y_{1}\right)=\operatorname{ind}\left(Y_{2}\right)=-1$, o resultado é imediato.

Agora, por hipótese de indução, assuma que o teorema é válido para todos os subespaços $Z_{1}$ e $Z_{2}$ de $X$ tais que $\operatorname{ind}\left(Z_{1}\right)+\operatorname{ind}\left(Z_{2}\right)=k$, para todo $k=-1,0, \ldots, m-1$. Mostremos que vale para $k=m$, ou seja, mostremos que, dados $Y_{1}$ e $Y_{2}$ subespaços de $X$ tais $\operatorname{ind}\left(Y_{1}\right)+\operatorname{ind}\left(Y_{2}\right)=m$, vale

$$
\operatorname{ind}\left(Y_{1} \cup Y_{2}\right) \leqslant m+1
$$

Para isso, precisamos mostrar que, dados $x \in Y_{1} \cup Y_{2}$ e $F$ um subconjunto fechado de $Y_{1} \cup Y_{2}$ que não contém $x$, existe uma $(\{x\}, F)$-partição $C$ em $Y_{1} \cup Y_{2}$ tal que ind $(C) \leqslant m$.

Sejam $x \in Y_{1} \cup Y_{2}$ e $F$ um subconjunto fechado de $Y_{1} \cup Y_{2}$ que não contém $x$. Assuma que $x \in Y_{1}$ e tome $E$ o fecho de uma vizinhança de $F$ tal que $x \notin E$. Como $\operatorname{ind}\left(Y_{1}\right)<\infty$, existe uma $\left(\{x\}, E \cap Y_{1}\right)$-partição $S$ em $Y_{1}$ tal que ind $(S) \leqslant \operatorname{ind}\left(Y_{1}\right)-1$.

Escreva $Y_{1} \backslash S=U \cup V$, em que $U$ e $V$ são abertos de $Y_{1}$ disjuntos tais que $x \in U$ e $E \cap Y_{1} \subset V$. Tome $W \subset X$ uma vizinhança (aberta) de $x$ tal que $\bar{W} \cap Y_{1} \subset U$.

Note que, em particular, $S$ é uma $\left(\bar{W} \cap Y_{1}, E \cap Y_{1}\right)$-partição em $Y_{1}$ e podemos reduzir $W$ de forma que $\bar{W} \cap E=\varnothing$. Logo, pelo Lema A.5, existe uma $(\{x\}, F)$-partição $T$ em $X$ tal que $T \cap Y_{1} \subset S$. 
Agora, do Lema B.5, obtemos que ind $\left(T \cap Y_{2}\right) \leqslant \operatorname{ind}\left(Y_{2}\right)$ e também

$$
\operatorname{ind}\left(T \cap Y_{1}\right) \leqslant \operatorname{ind}(S) \leqslant \operatorname{ind}\left(Y_{1}\right)-1
$$

de modo que, pela hipótese de indução,

$$
\operatorname{ind}\left(\left(T \cap Y_{1}\right) \cup\left(T \cap Y_{2}\right)\right) \leqslant\left(\operatorname{ind}\left(Y_{1}\right)-1\right)+\left(\operatorname{ind}\left(Y_{2}\right)\right)+1=m .
$$

Finalmente, basta ver que $C:=T \cap\left(Y_{1} \cup Y_{2}\right)$ é uma $(\{x\}, F)$-partição em $Y_{1} \cup Y_{2}$ com $\operatorname{ind}(C) \leqslant m$.

Corolário B.7. Se $X$ pode ser escrito como uma reunião de $m+1$ supespaços de dimensão topológica nula, então ind $(X) \leqslant m$.

\section{Demonstração:}

Basta combinar a Proposição B.4 e o Teorema B.6.

Lema B.8. São equivalentes:

a) para todo $x \in X$ e toda vizinhança $U$ de $x$, existe uma $(\{x\}, X \backslash U)$-partição $C$ tal que $\operatorname{dim}_{\mathrm{T}}(C) \leqslant m-1$;

b) para todo par de fechados disjuntos $F, G$ de $X$, existe uma $(F, G)$-partição $C$ tal que $\operatorname{dim}_{\mathrm{T}}(C) \leqslant m-1$.

\section{Demonstração:}

Que $b) \Rightarrow a$ ), é imediato.

$$
a) \Rightarrow b)
$$

Sejam $F, G \subset X$ fechados disjuntos. Precisamos construir uma $(F, G)$-partição $C$ tal que $\operatorname{dim}_{\mathrm{T}}(C) \leqslant m-1$.

Para cada $x \in X$, existem abertos disjuntos $U(x)$ e $V(x)$ tais que

i) $x \in U(x)$;

ii) pondo $L(x)=X \backslash(U(x) \cup V(x))$, tem-se $\operatorname{dim}_{\mathrm{T}}(L(x)) \leqslant m-1$; e 
iii) $\overline{U(x)} \cap F=\varnothing$ ou $\overline{U(x)} \cap G=\varnothing$.

De fato, basta proceder da seguinte maneira: se $x \in F$, tome uma vizinhança $U_{0}(x)$ de $x$ tal que $\overline{U_{0}(x)} \cap G=\varnothing$ e então aplique o item $a$ ) para $x$ e $U_{0}(x)$, de onde obtemos abertos disjuntos $U(x)$ e $V(x)$ tais que $x \in U(x), \operatorname{dim}_{\mathrm{T}}(X \backslash(U(x) \cup V(x))) \leqslant m-1 \mathrm{e}$ $\overline{U(x)} \cap G \subset \overline{U_{0}(x)} \cap G=\varnothing$. Analogamente, quando $x \in G$, obtemos $U(x)$ e $V(x)$ como antes, mas com $\overline{U(x)} \cap F=\varnothing$. Para $x \in X \backslash(F \cup G)$, basta proceder como no primeiro ou no segundo caso.

Uma vez que $X$ é separável, existem pontos $x_{1}, x_{2}, \ldots$ tais que $\left\{U\left(x_{n}\right): n \in \mathbb{N}\right\}$ é uma cobertura aberta enumerável, a qual pode ser descrita como reunião disjunta das coleções

$$
\mathcal{U}=\left\{U\left(x_{n}\right): n \in \mathbb{N}, \overline{U\left(x_{n}\right)} \cap F \neq \varnothing\right\}=\left\{U_{j}\right\}
$$

$\mathrm{e}$

$$
\mathcal{V}=\left\{U\left(x_{n}\right): n \in \mathbb{N}, \overline{U\left(x_{n}\right)} \cap F=\varnothing\right\}=\left\{V_{j}\right\}
$$

Considere os abertos disjuntos

$$
U=\bigcup_{i=1}^{\infty}\left(U_{i} \backslash \bigcup_{j<i} \overline{V_{j}}\right) \text { e } V=\bigcup_{i=1}^{\infty}\left(V_{i} \backslash \bigcup_{j \leqslant i} \overline{U_{j}}\right)
$$

e note que $F \subset U$ e $G \subset V$. Assim, $C:=X \backslash(U \cup V)$ é uma $(F, G)$-partição.

De fato, $U$ e $V$ são disjuntos, pois se existisse $x \in U \cap V$, então existiriam índices $i$ e $k$ tais que

$$
x \in\left(U_{i} \backslash \bigcup_{j<i} \overline{V_{j}}\right) \cap\left(V_{k} \backslash \bigcup_{j \leqslant k} \overline{U_{j}}\right) .
$$

Se $i \leqslant k$, então $x \in U_{i}$ e também $x \notin \overline{U_{i}}$, um absurdo. E analogamente para $i>k$.

Defina

$$
L=\bigcup_{n=1}^{\infty} L\left(x_{n}\right)
$$

de modo que, pelo Teorema 2.18 , temos $\operatorname{dim}_{\mathrm{T}}(L) \leqslant m-1$.

Mostremos que $C \subset L$.

Dado $y \in C$, seja $i$ o primeiro natural tal que $y \in \overline{U_{i}} \cup \overline{V_{i}}$.

Se $y \in \overline{U_{i}}$, então $y \notin U_{i}$, pois caso contrário, teríamos $x \in U_{i} \backslash \bigcup_{j<i} \overline{V_{j}}$, o que não pode ocorrer, já que $y \in C=X \backslash(U \cup V)$. Logo, $y \in L$. 
E se $y \notin \overline{U_{i}}$, então $y \notin V_{i}$, pois caso contrário, teríamos $y \in V_{i} \backslash \bigcup_{j \leqslant i} \overline{U_{j}} \subset V$ e isto contraria novamente a escolha de $y$ em $C$. Logo, $y \in L$.

Portanto, $\operatorname{dim}_{\mathrm{T}}(C) \leqslant \operatorname{dim}_{\mathrm{T}}(L) \leqslant m-1$, e isto encerra a demonstração.

Teorema B.9. Vale a igualdade

$$
\operatorname{dim}_{\mathrm{T}}(X)=\operatorname{ind}(X) .
$$

\section{Demonstração:}

Primeiro, mostremos que $\operatorname{dim}_{\mathrm{T}}(X) \leqslant \operatorname{ind}(X)$, assumindo que ind $(X)<\infty$.

Vamos proceder por indução, sem grandes surpresas. Se ind $(X)=0$, então aplicamos a Proposição B.4 e obtemos $\operatorname{dim}_{\mathrm{T}}(X)=0$.

Suponha por indução que a desigualdade seja válida para todos os espaços $Z$ com $\operatorname{ind}(Z) \leqslant k, \operatorname{com} k=1,2, \ldots, m-1$; e assuma que ind $(X)=m$.

Para cada $x \in X$ e cada vizinhança $U$ de $x$, existe uma $(\{x\}, X \backslash U)$-partição $L$ tal que $\operatorname{ind}(L) \leqslant m-1$. Da hipótese de indução, vemos que todas tais partições têm dimensão topológica no máximo $m-1$. Recorrendo ao Lema B.8 e ao Corolário A.8, concluímos que $\operatorname{dim}_{\mathrm{T}}(X) \leqslant m$.

Agora mostremos que $\operatorname{ind}(X) \leqslant \operatorname{dim}_{\mathrm{T}}(X) . \operatorname{Se} \operatorname{dim}_{\mathrm{T}}(X)=-1$ ou $\operatorname{dim}_{\mathrm{T}}(X)=\infty$, então não há o que provar; com isso, assuma que $\operatorname{dim}_{\mathrm{T}}(X)=m$, com $m \in \mathbb{Z}_{+}$.

Pelo Corolário A.7, $X$ é a reunião de até $m+1$ subespaços de dimensão topológica nula. Consequentemente, $\operatorname{ind}(X) \leqslant m=\operatorname{dim}_{\mathrm{T}}(X)$, graças ao Corolário B.7.

Lema B.10. Vale a desigualdade

$$
\operatorname{ind}(X) \leqslant \operatorname{Ind}(X)
$$

\section{Demonstração:}

Se $\operatorname{Ind}(X)=\infty$ ou se $\operatorname{Ind}(X)=-1$, não há o que provar. Assuma então que o lema seja válido para todos os espaços $Z \operatorname{com}-1 \leqslant \operatorname{Ind}(Z) \leqslant m-1, m \in \mathbb{Z}_{+}$; e assuma que $\operatorname{Ind}(X)=m$.

Sejam $x \in X$ e $U$ uma vizinhança de $x$. Uma vez que $\{x\}$ e $X \backslash U$ são subconjuntos de $X$ fechados disjuntos, existe uma $(\{x\}, X \backslash U)$-partição tal que $\operatorname{Ind}(L) \leqslant m-1$. A 
hipótese de indução então garante que ind $(L) \leqslant \operatorname{Ind}(L) \leqslant m-1$, de onde concluímos que $\operatorname{ind}(X) \leqslant m=\operatorname{Ind}(X)$.

Finalmente, podemos estabelecer o principal resultado deste Apêndice: The Coincidence Theorem.

Teorema B.11. Vale a igualdade

$$
\operatorname{ind}(X)=\operatorname{Ind}(X)=\operatorname{dim}_{\mathrm{T}}(X)
$$

\section{Demonstração:}

Combinando o Teorema B.9 e o Lema B.10, já sabemos que

$$
\operatorname{dim}_{\mathrm{T}}(X)=\operatorname{ind}(X) \leqslant \operatorname{Ind}(X)
$$

Basta então mostrar que $\operatorname{Ind}(X) \leqslant \operatorname{dim}_{\mathrm{T}}(X)$.

Não há o que provar se $\operatorname{dim}_{\mathrm{T}}(X)=\infty$ ou $\operatorname{dim}_{\mathrm{T}}(X)=-1$. Assuma então que a afirmação seja válida para todos os espaços $Z \operatorname{com} \operatorname{dim}_{\mathrm{T}}(Z) \leqslant k, \operatorname{com} k=-1, \ldots, m-1$; e assuma que $\operatorname{dim}_{\mathrm{T}}(X)=m$.

Uma vez que ind $(X)=\operatorname{dim}_{\mathrm{T}}(X)=m$, para cada $x \in X$ e cada fechado $F$ de $X$ que não contém $x$, existe uma $(\{x\}, F)$-partição $L$ tal que ind $(L) \leqslant m-1$. Novamente, pelo Teorema B.9, vemos que $\operatorname{dim}_{\mathrm{T}}(L) \leqslant m-1$.

Agora, pelo Lema B.8, para todo par de fechados disjuntos $F$ e $G$ em $X$, existe uma $(F, G)$-partição $L$ com $\operatorname{dim}_{\mathrm{T}}(L) \leqslant m-1$. Da hipótese de indução, vemos que todas estas tais partições têm grande dimensão indutiva no máximo $m-1$. Concluímos daí que

$$
\operatorname{Ind}(X) \leqslant m=\operatorname{dim}_{\mathrm{T}}(X)
$$

como queríamos. 
Apêndice

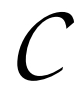

\section{Integração de Funções Não Mensuráveis}

Vamos apresentar um conceito abstrato de integração que generaliza a integração usual por se aplicar a funções não mensuráveis e ainda preservar importantes resultados como o Teorema da Convergência Monótona. As duas principais referências são Edgar [11] e Folland [18].

Fixemos $(X, \mathcal{M}, \mu)$ um espaço medida e $(\mathbb{R}, \mathcal{L}, m)$ o espaço de medida usual de Lebesgue da reta. Considere a família $\mathcal{A}$ das reuniões finitas disjuntas de conjuntos da forma

$$
E \times F
$$

em que $E \in \mathcal{M}$ e $F \in \mathcal{L}$; sabemos que $\mathcal{A}$ é uma álgebra em $X \times \mathbb{R}$.

Sendo assim, pomos em $X \times \mathbb{R}$ a $\sigma$-álgebra gerada por $\mathcal{A}$, a qual é chamada $\sigma$-álgebra produto e denotada, sem surpresas, por $\mathcal{M} \otimes \mathcal{L}$.

Os conjuntos $E \times F$ da álgebra $\mathcal{A}$, ditos retângulos (mensuráveis), satisfazem

$$
(A \times B) \cap(E \times F)=(A \cap E) \times(B \cap F)
$$

e também

$$
(E \times F)^{C}=\left(X \times F^{C}\right) \cup\left(E^{C} \times \mathbb{R}\right) .
$$

Temos um espaço produto $X \times \mathbb{R}$ e uma $\sigma$-álgebra produto $\mathcal{M} \otimes \mathcal{L}$; é de se esperar definir uma medida produto para tal par. 
Teorema C.1. Se $\left(f_{n}\right)_{n}$ é uma sequência finita ou infinita de funções mensuráveis de $X$ em $[0, \infty]$ e $f=\sum_{n} f_{n}$, então

$$
\int f=\sum_{n} \int f_{n}
$$

Consulte Folland [18].

Seja $E \times F$ um retângulo mensurável e suponha que possa ser escrito como uma reunião (finita ou apenas enumerável) disjunta de retângulos mensuráveis $E_{j} \times F_{j}$.

$\operatorname{Dados} x \in X$ e $t \in \mathbb{R}$

$$
\chi_{E}(x) \chi_{F}(t)=\chi_{E \times F}(x, t)=\sum_{j} \chi_{E_{j} \times F_{j}}(x, t)=\sum_{j} \chi_{E_{j}}(x) \chi_{F_{j}}(t) .
$$

Se integrarmos com respeito a $x$ e usar o Teorema C.1, obtemos

$$
\begin{aligned}
\mu(E) \chi_{F}(t) & =\int \chi_{E}(x) \chi_{F}(t) d \mu(x) \\
& =\sum_{j} \int \chi_{E_{j}}(x) \chi_{F_{j}}(t) d \mu(x) \\
& =\sum_{j} \mu\left(E_{j}\right) \chi_{F_{j}}(t),
\end{aligned}
$$

e agora, integrando em $t$

$$
\mu(E) m(F)=\sum_{j} \mu\left(E_{j}\right) m\left(F_{j}\right)
$$

Convencionando que $0 \cdot \infty=0$, defina

$$
\pi(G)=\sum_{j=1}^{n} \mu\left(E_{j}\right) m\left(F_{j}\right)
$$

para todo $G \in \mathcal{A}$ tal que $G$ é reunião disjunta de retângulos mensuráveis $E_{1} \times F_{1}, \ldots, E_{n} \times$ $F_{n}$. Desta forma, $\pi: \mathcal{A} \rightarrow[0, \infty]$ está bem definida em $\mathcal{A}$ (pois duas representações quaisquer de $G$ como reunião finita de retângulos mensuráveis têm um refinamento em comum) e é uma pré-medida em $\mathcal{A}$. 
Assim, dado $G \in \mathcal{A}$,

$$
\pi^{*}(G):=\inf \left\{\sum_{j=1}^{\infty} \pi\left(G_{j}\right): G_{j} \in \mathcal{A} \text { tais que } G \subset \bigcup_{j=1}^{\infty} G_{j}\right\}
$$

define uma medida exterior em $X \times \mathbb{R}$, dita a medida exterior produto de $\mu$ e $m$, cuja restrição à $\sigma$-álgebra produto $\mathcal{M} \otimes \mathcal{L}$ é uma medida que estende $\pi$, dita a medida produto de $\mu$ e $m$.

Vamos denotar por $\overline{\mu \times m}$ e $\mu \times m$, respectivamente, a medida exterior $\pi^{*}$ e a medida $\left.\pi^{*}\right|_{\mathcal{M} \otimes \mathcal{L}^{*}}$

Para mais detalhes sobre a construção de medidas exteriores, $\sigma$-álgebra produto e de medidas a partir de medidas exteriores, consulte Folland [18].

A noção de integração que vamos definir é a mais intuitiva possível: a área sob o gráfico de uma função $f: X \rightarrow[0, \infty]$. Se a função não for mensurável, teremos a integral superior de $f$.

Definição C.2. A integral superior de uma função $f: X \rightarrow[0, \infty]$ com respeito a $\mu$ é definida por

$$
\overline{\int f} \mu=\overline{\mu \times m}\left(\operatorname{Graf}_{f}\right)
$$

em que

$$
\operatorname{Graf}_{f}=\{(x, t) \in X \times \mathbb{R}: 0<t<f(x)\}
$$

é dito o subgráfico de $f$.

Se f é uma função mensurável, então $\operatorname{Graf}_{f}$ está em $\mathcal{M} \times \mathcal{B}_{\mathbb{R}}$ e então é $\overline{\mu \times m}$ mensurável. Neste caso, a integral superior é chamada simplesmente de integral de $f$ com respeito a $\mu$ e escrita como

$$
\int f \mu=(\mu \times m)\left(\operatorname{Graf}_{f}\right)
$$

Assim como nos textos tradicionais de integração, dada $f: X \rightarrow[-\infty, \infty]$ mensurável, podemos definir as partes positiva $f^{+}$e negativa $f^{-}$de $f$, de modo que $f=f^{+}-f^{-}$, e a integral de $f$ é definida por

$$
\int f \mu=\int f^{+} \mu-\int f^{-} \mu
$$


sempre que não tivermos $\infty-\infty$.

Se $\int f \mu$ existe como número real, dizemos que $f$ é integrável; e dado $E \in \mathcal{M}$, a integral de $f$ sobre $E$ é definida por

$$
\int_{E} f \mu=\int f \chi_{E} \mu
$$

Dizemos que $\phi: X \rightarrow[0, \infty]$ é uma função simples se é mensurável e é uma combinação linear finita das funções $\chi_{E_{j}}$, para alguma coleção finita de conjuntos $E_{j} \in \mathcal{M}$ :

$$
\phi=\sum a_{j} \chi_{E_{j}}
$$

Sua integral de Lebesgue é dada por

$$
\int \phi=\sum a_{j} \mu\left(E_{j}\right)
$$

em que omitimos a medida $\mu$ na notação para indicar que se trata da integral de Lebesgue, não da integral definida em C.2.

Para mostrar que esta noção de integral de fato generaliza a integral de Lebesgue, precisamos do Teorema de Fubini-Tonelli tradicional (Teorema 1.22).

Teorema C.3. Se $(X, \mathcal{M}, \mu)$ é um espaço de medida $\sigma$-finito e $f: X \rightarrow[0, \infty]$ é mensurável, então a noção de integral em C.2 coincide com a integral tradicional de Lebesgue.

Mais precisamente,

$$
(\mu \times m)\left(\operatorname{Graf}_{f}\right)=\sup \int \phi
$$

com o supremo sendo tomado sobre todas as funções simples $\phi$ tais que $0 \leqslant \phi \leqslant f$.

\section{Demonstração:}

Como $\operatorname{Graf}_{f}$ é definido como sendo o conjunto dos $(x, t) \in X \times \mathbb{R}$ tais que $0<t<f(x)$, note que

$$
\operatorname{Graf}_{f}=\left(F_{1} \circ F_{2}\right)^{-1}((0, \infty))
$$

em que $F_{1}(u, v)=u-v$ é Borel mensurável (por ser contínua) e $F_{2}(x, t)=(f(x), t)$ é Lebesgue mensurável. 
Assim, Graf $_{f}$ é mensurável e aplicando o Teorema 1.22, obtemos

$$
\begin{aligned}
(\mu \times m)\left(\operatorname{Graf}_{f}\right) & =\iint \chi_{\mathrm{Graf}_{f}} d(\mu \times m) \\
& =\iint \chi_{\mathrm{Graf}_{f}} d m d \mu \\
& =\int\left(\int \chi_{\operatorname{Graf}_{f}}(x, t) d m(t)\right) d \mu(x) \\
& =\int m(\{t \in[0, \infty): 0 \leqslant t<f(x)\}) d \mu(x) \\
& =\int f(x) d \mu(x) \\
& =\int f d \mu,
\end{aligned}
$$

no sentido de Lebesgue.

Naturalmente, poderíamos trocar $<$ por $\leqslant$ na definição de $\operatorname{Graf}_{f}$,

$$
\operatorname{Graf}_{f}=\{(x, t) \in X \times \mathbb{R}: 0<t \leqslant f(x)\},
$$

de modo que $\operatorname{Graf}_{f}=\left(F_{1} \circ F_{2}\right)^{-1}([0, \infty))$.

Com isso, sempre que estivermos nestas condições, os resultados coincidem os resultados usuais de integração de Lebesgue. No entanto, apresentamos generalizações que não exigem que o espaço seja $\sigma$-finito.

Teorema C.4. Sejam $f, g: X \rightarrow[0, \infty]$ funções.

1) se $f$ é da forma $\sum_{j=1}^{\infty} a_{j} \chi_{E_{j}}$, em que $E_{j}$ são disjuntos, então

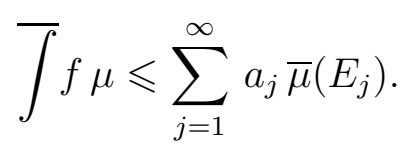

Se $E_{j} \in \mathcal{M}$, para todo $j$, de modo que $f$ é mensurável, então

$$
\int f \mu=\sum_{j=1}^{\infty} a_{j} \mu\left(E_{j}\right)
$$


2) se $f$ e g são não negativas, então

$$
\bar{\int}(f+g) \mu \leqslant \bar{\int} f \mu+\bar{\int} g \mu .
$$

3) se $f$ e $g$ são mensuráveis não negativas, então $f+g$ é mensurável e

$$
\int(f+g) \mu=\int f \mu+\int g \mu \text {. }
$$

4) se $f$ e g são integráveis, então $f+g$ é integrável $e$

$$
\int(f+g) \mu=\int f \mu+\int g \mu \text {. }
$$

5) se f é não negativa e $\lambda \geqslant 0$ é dado, então

$$
\bar{\int} \lambda f \mu=\lambda \bar{\int} f \mu .
$$

6) se $f$ é integrável e $\lambda \in \mathbb{R}$ é dado, então $\lambda f$ é mensurável $e$

$$
\int \lambda f \mu=\lambda \int f \mu
$$

\section{Demonstração:}

\section{Item 1)}

Por definição, sabemos que

$$
\bar{\mu}(E)=\inf \{\mu(F): F \in \mathcal{M} \operatorname{com} E \subset F\}
$$

Se $f=\chi_{E}$, então $\operatorname{Graf}_{f}=E \times(0,1)$; dado $F \supset E$ mensurável, temos que

$$
\overline{\mu \times m}\left(\operatorname{Graf}_{f}\right) \leqslant \overline{\mu \times m}(F \times(0,1))=\mu(F) m((0,1))=\mu(F)
$$


e tomando o ínfimo sobre todos conjuntos $F$ desta forma, obtemos

$$
\overline{\mu \times m}\left(\operatorname{Graf}_{f}\right) \leqslant \bar{\mu}(E) .
$$

Neste caso, se $E$ é mensurável e

$$
\operatorname{Graf}_{f}=E \times(0,1) \subset \bigcup_{j} A_{j} \times B_{j}
$$

em que $A_{j} \times B_{j}$ são retângulos mensuráveis, então

$$
\bar{\mu}(E)<\mu(E) m((0,1))=(\mu \times m)(E \times(0,1)) \leqslant \sum_{j}(\mu \times m)\left(A_{j} \times B_{j}\right),
$$

de modo que $\bar{\mu}(E) \leqslant \overline{\mu \times m}\left(\operatorname{Graf}_{f}\right)$.

Agora, assuma que

$$
f=\sum_{j=1}^{\infty} a_{j} \chi_{E_{j}}
$$

em que $E_{j}$ são disjuntos, então $\operatorname{Graf}_{f}=\{(x, t): 0<t<f(x)\}$ é a reunião disjunta dos retângulos $A_{j} \times\left(0, a_{j}\right)$, cuja medida exterior produto é $a_{j} \bar{\mu}\left(A_{j}\right)$; daí, por subaditividade, concluímos que

$$
\overline{\int f} \mu=\overline{\mu \times m}\left(\operatorname{Graf}_{f}\right)=\overline{\mu \times m}\left(\bigcup_{j} A_{j} \times\left(0, a_{j}\right)\right) \leqslant \sum_{j} a_{j} \bar{\mu}\left(A_{j}\right) .
$$

E se os conjuntos $A_{j}$ são mensuráveis, então os retângulos $A_{j} \times\left(0, a_{j}\right)$ são mensuráveis; agora a $\sigma$-aditividade da medida $\mu \times m$ garante que

$$
\overline{\int f \mu}=\sum_{j} a_{j} \bar{\mu}\left(A_{j}\right)
$$

Item 2)

Mostremos que $\overline{\mu \times m}\left(\operatorname{Graf}_{f+g}\right) \leqslant \overline{\mu \times m}\left(\operatorname{Graf}_{f}\right)+\overline{\mu \times m}\left(\operatorname{Graf}_{g}\right)$. 
Dados $A_{j} \times B_{j}$ e $C_{j} \times D_{j}$ retângulos mensuráveis tais que

$$
\operatorname{Graf}_{f} \subset \bigcup_{j} A_{j} \times B_{j} \text { e } \operatorname{Graf}_{g} \subset \bigcup_{j} C_{j} \times D_{j}
$$

basta mostrar que

$$
\overline{\mu \times m}\left(\operatorname{Graf}_{f+g}\right) \leqslant \sum_{j}(\mu \times m)\left(A_{j} \times B_{j}\right)+\sum_{j}(\mu \times m)\left(C_{j} \times D_{j}\right) .
$$

Para cada $n$, considere a união finita $\bigcup_{j=1}^{n} A_{j} \times B_{j}$, e então defina $f_{n}(x)$, para cada $x \in X$, como sendo

$$
f_{n}(x):=m\left\{t:(x, t) \in \bigcup_{j=1}^{n} A_{j} \times B_{j}\right\}
$$

daí $f_{n}: X \rightarrow[0, \infty]$ é uma função que assume finitos valores, cada qual é tomado em um conjunto mensurável de $\mathcal{M}$. Note que $\operatorname{Graf}_{f_{n}}$ é uma reunião finita de retângulos mensuráveis e $(\mu \times m)\left(\operatorname{Graf}_{f_{n}}\right) \leqslant \sum_{j=1}^{n}(\mu \times m)\left(A_{j} \times B_{j}\right)$.

Uma vez que $\bigcup_{j=1}^{n} A_{j} \times B_{j}$ formam uma sequência crescente de conjuntos encaixados e $\bigcup_{j=1}^{\infty} A_{j} \times B_{j}$ cobre $\operatorname{Graf}_{f}$, vemos que $\lim _{n \rightarrow \infty} f_{n}(x) \geqslant f(x)$.

Similarmente, definimos $g_{n}: X \rightarrow[0, \infty]$ por

$$
g_{n}(x):=m\left\{t:(x, t) \in \bigcup_{j=1}^{n} C_{j} \times D_{j}\right\}
$$

A soma $h_{n}=f_{n}+g_{n}$ também assume finitos valores e seu subgráfico $\operatorname{Graf}_{f_{n}+g_{n}}$ é uma reunião finita de retângulos mensuráveis, logo

$$
(\mu \times m)\left(\operatorname{Graf}_{f_{n}+g_{n}}\right)=(\mu \times m)\left(\operatorname{Graf}_{f_{n}}\right)+(\mu \times m)\left(\operatorname{Graf}_{g_{n}}\right) .
$$

Sendo assim, $\bigcup_{n} \operatorname{Graf}_{f_{n}+g_{n}}$ é uma reunião enumerável de retângulos mensuráveis que 
contêm $\operatorname{Graf}_{f+g}$, de modo que

$$
\begin{aligned}
\overline{\mu \times m}\left(\operatorname{Graf}_{f+g}\right) & \leqslant(\mu \times m)\left(\bigcup_{n} \operatorname{Graf}_{f_{n}+g_{n}}\right) \\
& =\lim _{n \rightarrow \infty}(\mu \times m)\left(\operatorname{Graf}_{f_{n}+g_{n}}\right) \\
& =\lim _{n \rightarrow \infty}\left((\mu \times m)\left(\operatorname{Graf}_{f_{n}}\right)+(\mu \times m)\left(\operatorname{Graf}_{g_{n}}\right)\right) \\
& \leqslant \sum_{j=1}^{n}(\mu \times m)\left(A_{j} \times B_{j}\right)+\sum_{j=1}^{n}(\mu \times m)\left(C_{j} \times D_{j}\right),
\end{aligned}
$$

como queríamos.

\section{Item 3)}

Suponha que $f$ e $g$ são funções mensuráveis não negativas, daí existem sequências crescentes de funções simples não negativas $\left(f_{n}\right)_{n}$ e $\left(g_{n}\right)_{n}$ tais que $f_{n} \rightarrow f$ e $g_{n} \rightarrow g$ pontualmente. Com isso, os subgráficos $\operatorname{Graf}_{f_{n}+g_{n}}$ formam uma sequência crescente de conjuntos encaixados rumo ao subgráfico de $f+g$, logo

$$
\begin{aligned}
\int(f+g) \mu & =(\mu \times m)\left(\operatorname{Graf}_{f+g}\right) \\
& =\lim _{n \rightarrow \infty}(\mu \times m)\left(\operatorname{Graf}_{f_{n}+g_{n}}\right) \\
& =\lim _{n \rightarrow \infty} \int\left(f_{n}+g_{n}\right) \mu \\
& =\lim _{n \rightarrow \infty}\left(\int f_{n} \mu+\int g_{n} \mu\right), \text { pois } f_{n}+g_{n} \text { são funções simples, } \\
& =\lim _{n \rightarrow \infty}\left((\mu \times m)\left(\operatorname{Graf}_{f_{n}}\right)+(\mu \times m)\left(\operatorname{Graf}_{g_{n}}\right)\right) \\
& =\int f \mu+\int g \mu .
\end{aligned}
$$

Item 4)

Agora, suponha que $f$ e $g$ são funções integráveis. Seja $h=f+g$, daí

$$
h^{+}+f^{-}+g^{-}=h^{-}+f^{+}+g^{+},
$$


e pelo item 3), sabemos que

$$
\int h^{+} \mu+\int f^{-} \mu+\int g^{-} \mu=\int h^{-} \mu+\int f^{+} \mu+\int g^{+} \mu
$$

e da integrabilidade de $f$ e $g$, obtemos

$$
\int h^{+} \mu-\int h^{-} \mu=\int f^{+} \mu-\int f^{-} \mu+\int g^{+} \mu-\int g^{-} \mu
$$

ou equivalentemente,

$$
\int h \mu=\int f \mu+\int g \mu
$$

Ítens 5) e 6)

Dados $\lambda>0$ e $A_{j} \times B_{j}$ um retângulo mensurável de $X \times \mathbb{R}$, sabemos que $A_{j} \times\left(\lambda B_{j}\right)$ é também um retângulo mensurável e que $(\mu \times m)\left(A_{j} \times\left(\lambda B_{j}\right)\right)=\lambda\left((\mu \times m)\left(A_{j} \times B_{j}\right)\right)$.

Se $\operatorname{Graf}_{f} \subset \bigcup_{j} A_{j} \times B_{j}$, então $\operatorname{Graf}_{\lambda f} \subset \bigcup_{j} A_{j} \times\left(\lambda B_{j}\right)$, o que encerra a demonstração usando os ítens anteriores.

Teorema C.5. (Teorema da Convergência Monótona para Integrais Superiores)

Se $f_{n}: X \rightarrow[0, \infty]$ formam uma sequência de funções que crescem pontualmente, i.e., $f_{1}(x) \leqslant f_{2}(x) \leqslant \ldots$, para todo $x \in X$, então

$$
\bar{\int} \lim _{n \rightarrow \infty} f_{n} \mu=\lim _{n \rightarrow \infty} \bar{\int} f_{n} \mu .
$$

\section{Demonstração:}

Uma vez que

$$
G_{n}=\left\{(x, t) \in X \times \mathbb{R}: 0<t<f_{n}(x)\right\}
$$

formam uma sequência crescente de conjuntos encaixados cuja reunião é

$$
G=\left\{(x, t) \in X \times \mathbb{R}: 0<t<\lim _{n \rightarrow \infty} f_{n}(x)\right\}
$$

a continuidade inferior de $\overline{\mu \times m}$ implica que

$$
\lim _{n \rightarrow \infty} \overline{\mu \times m}\left(G_{n}\right)=\overline{\mu \times m}(G) .
$$


Corolário C.6. Dadas funções $f_{n}: X \rightarrow[0, \infty]$, vale

$$
\bar{\int} \sum_{n=1}^{\infty} f_{n} \mu \leqslant \sum_{n=1}^{\infty} \bar{\int} f_{n} \mu .
$$

E se $f_{n}$ são todas mensuráveis, então

$$
\int \sum_{n=1}^{\infty} f_{n} \mu=\sum_{n=1}^{\infty} \int f_{n} \mu
$$

\section{Demonstração:}

Basta aplicar o Teorema C.5 à sequência de somas parciais $\sum_{n=1}^{N} f_{n}(x)$ e usar subaditividade da integral superior (Teorema C.4).

E quando $f_{n}$ são mensuráveis, também pelo Teorema C.4 temos a igualdade.

Os resultados apresentados são o ferramental necessário para justificar a argumentação na Seção 2.4. No entanto, vale apontar que toda uma nova teoria de integração segue daqui por meio desta noção de integral superior. Profundos resultados de integração se mantém: Teorema da Mudança de Variáveis, Lema de Fatou, Teorema da Convergência Dominada, Teorema da Média Quadrática Limitada, Teorema de Fubini-Tonelli, Teorema de Lebesgue-Radon-Nikodym, Teorema da Representação de Riesz, e outros.

Para consultá-los, veja Edgar [11]. 
Apêndice

\section{Medidas de Haar}

Neste apêndice, nos concentramos em mostrar que existe uma constante $c_{m}$ real positiva tal que

$$
\mathcal{L}^{m}(A)=c_{m} \mu^{(m)}(A)
$$

para todo $A \subset \mathbb{R}^{m}$ mensurável. Em outras palavras, em $\mathbb{R}^{m}$, as medidas de Hausdorff e de Lebesgue são iguais, a menos de um ajuste por uma constante $c_{m}$, a qual de fato vem a ser o volume de uma bola de diâmetro unitário.

A abordagem que apresentamos é baseada em Folland [18], porém particularizada a $\mathbb{R}^{m}$, por praticidade. Em sua maior generalidade, trataríamos abstratamente de grupos topológicos e, neste contexto, definiríamos medidas de Haar e veríamos que a relação acima de fato vale. Consulte Folland [18] para conferir tal abordagem.

Vamos usar a notação $C_{\mathrm{c}}\left(\mathbb{R}^{m}\right)$ para indicar o conjunto das funções $f: \mathbb{R}^{m} \rightarrow \mathbb{R}$ contínuas de suporte compacto.

Definição D.1. Uma medida de Radon $\mu$ em $\mathbb{R}^{m}$ é uma medida de Borel que é finita em compactos e é regular, i.e., satisfaz

$$
\mu(E)=\inf \{\mu(U): U \supset E \text { é aberto }\}
$$

$e$

$$
\mu(E)=\sup \{\mu(K): K \subset E \text { é compacto }\}
$$


para todo boreliano $E \subset \mathbb{R}^{m}$.

Se uma medida $\mu \mathrm{em} \mathbb{R}^{m}$ satisfaz (D.0.2), é dita uma medida exteriormente regular, bem como se satisfaz (D.0.3), é dita uma medida interiormente regular.

Além de ser instrumento para a construção de medidas, o Teorema da Representação de Riesz para medidas de Radon fornece uma importante conexão entre a Teoria da Medida e a Análise Funcional, pois caracteriza certos funcionais lineares contínuos em termos de integração com respeito a uma medida de Radon, de maneira biunívoca.

\section{Proposição D.2. (Folland [18], Proposition 4.41)}

Se $K \subset \mathbb{R}^{m}$ é um compacto e $U_{1}, \ldots, U_{n}$ é uma cobertura aberta (finita) de $K$, então existe uma partição da unidade $\left\{g_{1}, \ldots, g_{n}\right\}$ em $K$ subordinada à cobertura $\left\{U_{1}, \ldots, U_{n}\right\}$ tal que $\operatorname{supp}\left(g_{j}\right) \subset U_{j}$ é compacto, para todo $j=1, \ldots, n$.

\section{Demonstração:}

Para cada $x \in K$, tome $r_{x}>0$ tal que $B_{\mathbb{R}^{m}}\left(x, r_{x}\right) \subset B_{\mathbb{R}^{m}}\left[x, r_{x}\right] \subset U_{j}$, para algum $j$, de modo que tais bolas formam uma cobertura aberta para $K$.

Sejam $x_{1}, \ldots, x_{n} \in K$ tais que

$$
K \subset \bigcup_{j=1}^{n} B_{\mathbb{R}^{m}}\left[x_{j}, r_{x_{j}}\right]
$$

Defina $F_{j} \subset U_{j}$ como sendo a reunião das bolas fechadas $B_{\mathbb{R}^{m}}\left[x_{j}, r_{x_{j}}\right]$ contidas em $U_{j}$, logo um compacto. Pelo Lema de Urysohn (Lema 1.7), existem $g_{1}, \ldots, g_{n} \in C_{\mathrm{c}}\left(\mathbb{R}^{m},[0,1]\right)$ $\left.\operatorname{com} g_{j}\right|_{F_{j}} \equiv 1 \operatorname{esupp}\left(g_{j}\right) \subset U_{j}$.

Como os conjuntos $F_{j}$ cobrem $K$, temos que $\sum_{j=1}^{n} g_{j}(x) \geqslant 1$ em $K$. Novamente, pelo Lema de Urysohn (Lema 1.7), podemos escolher $f \in C_{\mathrm{c}}\left(\mathbb{R}^{m},[0,1]\right)$ tal que $\left.f\right|_{K} \equiv 1$ e $\operatorname{supp}(f) \subset\left\{x \in \mathbb{R}^{m}: \sum_{j=1}^{n} g_{j}(x)>0\right\}$.

Defina $g_{n+1}=1-f$, de modo que $h:=\sum_{j=1}^{n+1} g_{j}>0$ em $\mathbb{R}^{m}$, e também

$$
h_{j}(x)=\frac{g_{j}(x)}{h(x)}
$$


para cada $j=1, \ldots, n$. Com isso, $\operatorname{supp}\left(h_{j}\right)=\operatorname{supp}\left(g_{j}\right) \subset U_{j}$ e $\sum_{j=1}^{n} h_{j} \equiv 1$ em $K$.

\section{Teorema D.3. (Representação de Riesz para medidas de Radon)}

Seja $T: C_{\mathrm{c}}\left(\mathbb{R}^{m}\right) \rightarrow \mathbb{R}$ é um funcional linear positivo, i.e., $T(f) \geqslant 0$ sempre que $f \geqslant 0$.

Então, existe uma única medida de Radon $\mu$ em $\mathbb{R}^{m}$ tal que

$$
T(f)=\int f d \mu
$$

para toda $f \in C_{\mathrm{c}}\left(\mathbb{R}^{m}\right)$.

Além disso, dados $U \subset \mathbb{R}^{m}$ aberto e $K \subset \mathbb{R}^{m}$ compacto, valem

$$
\mu(U)=\sup \left\{T(f): f \in C_{\mathrm{c}}\left(\mathbb{R}^{m}\right) \operatorname{com} 0 \leqslant f \leqslant 1 e \operatorname{supp}(f) \subset U\right\}
$$

$e$

$$
\mu(K)=\inf \left\{T(f): f \in C_{\mathrm{c}}\left(\mathbb{R}^{m}\right) \text { com } f \geqslant \chi_{K}\right\}
$$

\section{Demonstração:}

Unicidade:

Seja $\mu$ uma medida de Radon tal que

$$
T(f)=\int f d \mu
$$

para toda $f \in C_{\mathrm{c}}\left(\mathbb{R}^{m}\right)$.

Dado um aberto $U \subset \mathbb{R}^{m}$, claro que $T(f) \leqslant \mu(U)$, sempre que $f \in C_{\mathrm{c}}\left(\mathbb{R}^{m}\right)$ satisfaz $0 \leqslant f \leqslant 1$ e $\operatorname{supp}(f) \subset U$.

Por outro lado, se $K \subset U$ é um compacto, do Lema de Urysohn (Lema 1.7), existe $f \in C_{\mathrm{c}}\left(\mathbb{R}^{m}\right)$ tal que $0 \leqslant f \leqslant 1, \operatorname{supp}(f) \subset U$ e $\left.f\right|_{K} \equiv 1$, daí $\mu(K) \leqslant \int f d \mu=T(f)$. 
Sendo $\mu$ uma medida regular, temos

$$
\begin{aligned}
\mu(U) & =\sup \{\mu(K): K \subset U \text { é compacto }\} \\
& \leqslant \sup \left\{T(f): f \in C_{\mathrm{c}}\left(\mathbb{R}^{m}\right), 0 \leqslant f \leqslant 1, \operatorname{supp}(f) \subset U \text { e }\left.f\right|_{K} \equiv 1\right\} \\
& \leqslant \sup \left\{T(f): f \in C_{\mathrm{c}}\left(\mathbb{R}^{m}\right), 0 \leqslant f \leqslant 1 \operatorname{esupp}(f) \subset U\right\} \\
& \leqslant \mu(U),
\end{aligned}
$$

ou seja, vale (D.0.4) . Assim, $\mu$ é determinada pelo funcional $T$ em todos os abertos de $\mathbb{R}^{m}$, consequentemente, em todos os conjuntos de Borel de $\mathbb{R}^{m}$, pois $\mu$ é regular.

Se $\nu$ é uma outra medida de Radon que satisfaz $T(f)=\int f d \nu$, para toda $f \in C_{\mathrm{c}}\left(\mathbb{R}^{m}\right)$, então também satisfaz (D.0.4), $\operatorname{logo} \mu$ e $\nu$ coincidem.

Quanto à existência, o argumento acima sugere definirmos

$$
\mu(U)=\sup \left\{T(f): f \in C_{\mathrm{c}}\left(\mathbb{R}^{m}\right) \operatorname{com} 0 \leqslant f \leqslant 1 \operatorname{esupp}(f) \subset U\right\}
$$

para os abertos $U \subset \mathbb{R}^{m}$, e

$$
\mu^{*}(E)=\inf \{\mu(U): U \supset E \text { é aberto }\}
$$

para subconjuntos $E \subset \mathbb{R}^{m}$ quaisquer. E note que $\mu^{*}(U)=\mu(U)$, com $U$ aberto, pois $\mu^{*}(U) \leqslant \mu(U) \leqslant \mu(W)$, para todo aberto $W \supset U$.

$\mu^{*}$ é uma medida exterior

Seja $\left\{U_{j}\right\}_{j \in \mathbb{N}}$ uma coleção enumerável de abertos. Se mostrarmos que

$$
\mu\left(\bigcup_{j=1}^{\infty} U_{j}\right) \leqslant \sum_{j=1}^{\infty} \mu\left(U_{j}\right)
$$

então, dado $E \subset \mathbb{R}^{m}$ qualquer, temos

$$
\mu^{*}(E)=\inf \left\{\sum_{j=1}^{\infty} \mu\left(U_{j}\right): U_{j} \text { é aberto e } E \subset \bigcup_{j=1}^{\infty} U_{j}\right\}
$$

a qual define uma medida exterior. 
Sejam $U=\bigcup_{j=1}^{\infty} U_{j}$ e $f \in C_{\mathrm{c}}\left(\mathbb{R}^{m}\right)$ tal que $0 \leqslant f \leqslant 1$ e $K:=\operatorname{supp}(f) \subset U$, de modo que, a menos de reordenação dos abertos $U_{j}$,

$$
K \subset \bigcup_{j=1}^{n} U_{j}
$$

para algum $n$ natural.

Da Proposição D.2, existem $g_{1}, \ldots, g_{n} \in C_{\mathrm{c}}\left(\mathbb{R}^{m}\right)$ tais que $0 \leqslant g_{j} \leqslant 1, \operatorname{supp}\left(g_{j}\right) \subset U_{j}$ e $\sum_{j=1}^{n} g_{j} \equiv 1$ em $K$. Como $f=\sum_{j=1}^{n} f g_{j}, 0 \leqslant f g_{j} \leqslant 1$ e $\operatorname{supp}\left(f g_{j}\right) \subset U_{j}$, vemos que

$$
T(f)=\sum_{j=1}^{n} T\left(f g_{j}\right) \leqslant \sum_{j=1}^{n} \mu\left(U_{j}\right) \leqslant \sum_{j=1}^{\infty} \mu\left(U_{j}\right)
$$

Sendo arbitrária a função $f \in C_{\mathrm{c}}\left(\mathbb{R}^{m}\right)$ tal que $0 \leqslant f \leqslant 1$ e $\operatorname{supp}(f) \subset U$, segue que

$$
\mu(U) \leqslant \sum_{j=1}^{\infty} \mu\left(U_{j}\right)
$$

como queríamos.

Todo aberto é $\mu^{*}$-mensurável

Mostremos que, dados $U$ aberto e $E$ qualquer, vale

$$
\mu^{*}(E) \geqslant \mu^{*}(E \cap U)+\mu^{*}(E \backslash U)
$$

De início, suponha que $E$ é aberto e tome $\varepsilon>0$.

Como $E \cap U$ é aberto, existe $f \in C_{\mathrm{c}}\left(\mathbb{R}^{m}\right)$ tal que $0 \leqslant f \leqslant 1$, $\operatorname{supp}(f) \subset E \cap U$ e

$$
T(f)>\mu(E \cap U)-\varepsilon \text {. }
$$

Agora, como também $E \backslash \operatorname{supp}(f)$ é aberto, existe $g \in C_{\mathrm{c}}\left(\mathbb{R}^{m}\right)$ tal que $0 \leqslant g \leqslant 1$, $\operatorname{supp}(g) \subset E \backslash \operatorname{supp}(f)$ e

$$
T(g)>\mu(E \backslash \operatorname{supp}(f))-\varepsilon .
$$


Com isso, $0 \leqslant f+g \leqslant 1$ e $\operatorname{supp}(f+g) \subset E, \operatorname{logo}$

$$
\begin{aligned}
\mu(E) & \geqslant T(f)+T(g) \\
& >\mu(E \cap U)+\mu(E \backslash \operatorname{supp}(f))-2 \varepsilon \\
& \geqslant \mu^{*}(E \cap U)+\mu^{*}(E \backslash U)-2 \varepsilon .
\end{aligned}
$$

Fazendo $\varepsilon \rightarrow 0$, obtemos a desigualdade desejada. Para o caso geral, se $\mu^{*}(E)<\infty$, existe um aberto $V \supset E$ tal que $\mu(V)<\mu^{*}(E)+\varepsilon$, daí

$$
\begin{aligned}
\mu^{*}(E)+\varepsilon & >\mu(V) \\
& \geqslant \mu^{*}(V \cap U)+\mu^{*}(V \backslash U) \\
& \geqslant \mu^{*}(E \cap U)+\mu^{*}(E \backslash U)
\end{aligned}
$$

e novamente basta fazer $\varepsilon \rightarrow 0$.

Pelo Teorema de Carathéodory (Teorema 1.20), todo conjunto de Borel é $\mu^{*}$-mensurável e $\mu=\left.\mu^{*}\right|_{\mathcal{B}_{\mathbb{R}}}$ é uma medida de Borel. Além disso, $\mu$ é exteriormente regular e, por definição, satisfaz (D.0.4).

$\mu$ satisfaz (D.0.5) e é de Radon

Seja $K$ um compacto de $\mathbb{R}^{m}$.

Dada $f \in C_{\mathrm{c}}\left(\mathbb{R}^{m}\right)$ tal que $f \geqslant \chi_{K}$, considere o aberto $U_{\varepsilon}=\left\{x \in \mathbb{R}^{m}: f(x)>1-\varepsilon\right\}$. Se $g \in C_{\mathrm{c}}\left(\mathbb{R}^{m}\right)$ é tal que $0 \leqslant g \leqslant 1$ e $\operatorname{supp}(g) \subset U_{\varepsilon}$, então $(1-\varepsilon)^{-1} f-g \geqslant 0$ e

$$
T(g) \leqslant(1-\varepsilon)^{-1} T(f),
$$

$\log 0 \mu(K) \leqslant \mu\left(U_{\varepsilon}\right) \leqslant(1-\varepsilon)^{-1} T(f)$. Fazendo $\varepsilon \rightarrow 0$, obtemos $\mu(K) \leqslant T(f)$.

Por outro lado, dado $U \supset K$, do Lema de Urysohn (Lema 1.7), existe $f \in C_{\mathrm{c}}\left(\mathbb{R}^{m}\right.$ ) tal que $f \geqslant \chi_{K}, 0 \leqslant f \leqslant 1$ e $\operatorname{supp}(f) \subset U$, daí $T(f) \leqslant \mu(U)$. Como $\mu$ é uma medida exteriormente regular em $K$, vale (D.0.5), i.e.,

$$
\mu(K)=\inf \left\{T(f): f \in C_{\mathrm{c}}\left(\mathbb{R}^{m}\right) \operatorname{com} f \geqslant \chi_{K}\right\} .
$$

Em particular, $\mu$ é finita em compactos de $\mathbb{R}^{m}$.

Além disso, $\mu$ é também interiormente regular. 
De fato, dados $U \subset \mathbb{R}^{m}$ aberto e $\alpha<\mu(U)$, tome $g \in C_{\mathrm{c}}\left(\mathbb{R}^{m}\right)$ tal que $0 \leqslant g \leqslant 1$, $K:=\operatorname{supp}(g) \subset U$ e $T(g)>\alpha$.

Para toda $f \in C_{\mathrm{c}}\left(\mathbb{R}^{m}\right)$ tal que $f \geqslant \chi_{K}$, temos $f-g \geqslant 0$, daí $T(f) \geqslant T(g)>\alpha$. Agora, aplicando (D.0.5), obtemos $\mu(K)>\alpha$.

Em resumo, mostramos que, para todo $\alpha<\mu(U)$, existe um compacto $K \subset U$ tal que $\alpha<\mu(K) \leqslant \mu(U)$.

Portanto, $\mu$ é uma medida regular.

$T(f)=\int f d \mu$, para toda $f \in C_{\mathrm{c}}\left(\mathbb{R}^{m}\right)$

Uma vez que $C_{\mathrm{c}}\left(\mathbb{R}^{m}\right)$ é um subespaço linear gerado por $C_{\mathrm{c}}\left(\mathbb{R}^{m},[0,1]\right)$, basta mostrarmos que $T(f)=\int f d \mu$, para toda $f \in C_{\mathrm{c}}\left(\mathbb{R}^{m},[0,1]\right)$.

Fixado $N$ natural, defina os conjuntos $K_{0}=\operatorname{supp}(f)$ e $K_{j}=\left\{x \in \mathbb{R}^{m}: f(x) \geqslant j / N\right\}$, para cada $j=1, \ldots, N$, de modo que $K_{0} \supset K_{1} \supset K_{2} \supset \cdots \supset K_{N-1} \supset K_{N}$.

Defina também as funções $f_{1}, \ldots, f_{N} \in C_{\mathrm{c}}\left(\mathbb{R}^{m}\right)$ por

$$
f_{j}(x)=\left\{\begin{aligned}
0, & \text { se } x \notin K_{j-1} \\
f(x)-(j-1) / N, & \text { se } x \in K_{j-1} \backslash K_{j} \\
1 / N, & \text { se } x \in K_{j}
\end{aligned}\right.
$$

ou equivalentemente,

$$
f_{j}=\min \left\{\max \left\{f-\frac{j-1}{N}, 0\right\}, \frac{1}{N}\right\},
$$

de modo que $N^{-1} \chi_{K_{j}} \leqslant f_{j} \leqslant N^{-1} \chi_{K_{j-1}}$, daí

$$
\frac{1}{N} \mu\left(K_{j}\right) \leqslant \int f_{j} d \mu \leqslant \frac{1}{N} \mu\left(K_{j-1}\right)
$$

Note que, se $U$ é um aberto que contém $K_{j-1}$, então $N f_{j} \in C_{\mathrm{c}}\left(\mathbb{R}^{m}\right), 0 \leqslant N f_{j} \leqslant 1$, $\operatorname{supp}\left(N f_{j}\right) \subset U$ e $T\left(f_{j}\right) \leqslant N^{-1} \mu(U)$. Por (D.0.5) e por $\mu$ ser exteriormente regular, obtemos

$$
\frac{1}{N} \mu\left(K_{j}\right) \leqslant T\left(f_{j}\right) \leqslant \frac{1}{N} \mu\left(K_{j-1}\right)
$$


Além disso, como $f=\sum_{j=1}^{N} f_{j}$, segue que

$$
\frac{1}{N} \sum_{j=1}^{N} \mu\left(K_{j}\right) \leqslant \int f d \mu \leqslant \frac{1}{N} \sum_{j=0}^{N-1} \mu\left(K_{j}\right)
$$

e também

$$
\frac{1}{N} \sum_{j=1}^{N} \mu\left(K_{j}\right) \leqslant T(f) \leqslant \frac{1}{N} \sum_{j=0}^{N-1} \mu\left(K_{j}\right)
$$

Sendo assim,

$$
\left|T(f)-\int f \mu\right| \leqslant \frac{\mu\left(K_{0}\right)-\mu\left(K_{N}\right)}{N} \leqslant \frac{\mu(\operatorname{supp}(f))}{N} .
$$

Uma vez que $\mu(\operatorname{supp}(f))<\infty$ e $N$ é arbitrário, concluímos que

$$
T(f)=\int f d \mu
$$

Estamos reunindo resultados a fim de estabelecer a relação (D.0.1) entre a medida de Lebesgue em $\mathbb{R}^{m}$ e a $m$-medida de Hausdorff em $\mathbb{R}^{m}$. A abordagem que seguimos se faz valer do fato de ambas serem medidas de Radon. Os próximos resultados estabelecem critérios para que uma medida de Borel seja uma medida de Radon.

Proposição D.4. Sejam $\mu$ uma medida de Radon em $\mathbb{R}^{m}$ e $E$ um boreliano de $\mathbb{R}^{m}$, então

a) para cada $\varepsilon>0$, existem um aberto $U$ e um fechado $F$ tais que $F \subset E \subset U$ e $\mu(U \backslash F)<\varepsilon$.

b) existem um conjunto $F_{\sigma}, A$, e um conjunto $G_{\delta}, B$, de tal modo que $A \subset E \subset B$ e $\mu(B \backslash A)=0$.

\section{Demonstração:}

Sejam $E_{j} \subset \mathbb{R}^{m}$ conjuntos disjuntos tais que $\mu\left(E_{j}\right)<\infty$ e

$$
E=\bigcup_{j=1}^{\infty} E_{j}
$$


Para cada $j$, tome um aberto $U_{j} \supset E_{j}$ tal que $\mu\left(U_{j}\right)<\mu\left(E_{j}\right)+\varepsilon 2^{-j-1}$, e defina $U=\bigcup_{j=1}^{\infty} U_{j}$, de modo que $U \supset E$ é aberto e

$$
\mu(U \backslash E) \leqslant \mu\left(\bigcup_{j=1}^{\infty}\left(U_{j} \backslash E_{j}\right)\right) \leqslant \sum_{j=1}^{\infty} \mu\left(U_{j} \backslash E_{j}\right)<\varepsilon / 2
$$

Repetindo o argumento para $E^{C}$, obtemos um aberto $V \supset E^{C}$ tal que $\mu\left(V \backslash E^{C}\right)<\varepsilon / 2$. Com isso, $F:=V^{C} \subset E$ é um fechado e vale

$$
\mu(U \backslash F)=\mu(U \backslash E)+\mu(E \backslash F)=\mu(U \backslash E)+\mu\left(V \backslash E^{C}\right)<\varepsilon .
$$

Quanto ao item $b$ ), para cada $n$ natural, tome um aberto $U_{n}$ e um fechado $F_{n}$ tais que $F_{n} \subset E \subset U_{n}$ e $\mu\left(U_{n} \backslash F_{n}\right)<1 / n$. Defina

$$
A=\bigcup_{k=1}^{\infty} F_{n} \text { e } B=\bigcap_{n=1}^{\infty} U_{n}
$$

Assim,

$$
B \backslash A=\left(\bigcap_{n=1}^{\infty} U_{n}\right) \backslash\left(\bigcup_{k=1}^{\infty} F_{k}\right)=\bigcap_{n=1}^{\infty}\left\{U_{n} \cap\left(\bigcap_{k=1}^{\infty} F_{k}^{C}\right)\right\} \subset \bigcap_{n=1}^{\infty}\left(U_{n} \backslash F_{n}\right) \subset U_{n} \backslash F_{n},
$$

para todo $n$ natural. Consequentemente,

$$
\mu(B \backslash A) \leqslant \mu\left(U_{n} \backslash F_{n}\right)<1 / n, \forall n .
$$

Teorema D.5. Toda medida de Borel em $\mathbb{R}^{m}$ que é finita em compactos é uma medida de Radon em $\mathbb{R}^{m}$.

\section{Demonstração:}

Seja $\mu$ uma medida de Borel em $\mathbb{R}^{m}$ que é finita em compactos e note que $C_{\mathrm{c}}\left(\mathbb{R}^{m}\right) \subset$ $L^{1}(\mu)$. Com isso, a aplicação $T: C_{\mathrm{c}}\left(\mathbb{R}^{m}\right) \rightarrow \mathbb{R}$, definida por

$$
T(f):=\int f d \mu
$$


é um funcional linear positivo.

Pelo Teorema da Representação de Riesz (Teorema D.3), seja $\nu$ a medida de Radon associada a $T$.

Mostremos que $\mu$ é uma medida regular, consequentemente uma medida de Radon. Novamente do Teorema D.3, concluímos que $\mu=\nu$.

Dado um aberto $U$, tome compactos $K_{j}$ tais que $U=\bigcup_{j=1}^{\infty} K_{j}$. Seja $f_{1} \in C_{\mathrm{c}}\left(\mathbb{R}^{m}\right)$ tal que $0 \leqslant f \leqslant 1, \operatorname{supp}\left(f_{1}\right) \subset U$ e $f \equiv 1$ em $K_{1}$. Procedendo indutivamente, para cada $n>1$, seja $f_{n} \in C_{\mathrm{c}}\left(\mathbb{R}^{m}\right)$ tal que $0 \leqslant f_{n} \leqslant 1, \operatorname{supp}\left(f_{n}\right) \subset U$ e $f_{n} \equiv 1$ em $K_{1} \cup \cdots \cup K_{n}$ e $\operatorname{em} \operatorname{supp}\left(f_{1}\right) \cup \cdots \cup \operatorname{supp}\left(f_{n-1}\right)$.

Sendo assim, $\left(f_{n}\right)_{n}$ é uma sequência de funções que crescem pontualmente para $\chi_{U}$, daí pelo Teorema da Convergência Monótona, segue que

$$
\mu(U)=\lim _{n \rightarrow \infty} \int f_{n} d \mu=\lim _{n \rightarrow \infty} T\left(f_{n}\right)=\lim _{n \rightarrow \infty} \int f_{n} d \nu=\nu(U)
$$

Agora, dados $\varepsilon>0$ e um boreliano $E \subset \mathbb{R}^{m}$, pela Proposição D.4, existem um aberto $V \supset E$ e um fechado $F \subset E$ tais que $\nu(V \backslash F)<\varepsilon$.

Uma vez que $V \backslash F$ é um aberto, temos que $\mu(V \backslash F)=\nu(V \backslash F)<\varepsilon$, daí

$$
\mu(V)<\mu(F)+\varepsilon \leqslant \mu(E)+\varepsilon,
$$

$\operatorname{logo} \mu$ é uma medida exteriormente regular.

E como $F$ é reunião crescente de certos compactos $K_{j}$, temos que

$$
\lim _{j \rightarrow \infty} \mu\left(K_{j}\right)=\mu(F) \geqslant \mu(E)-\varepsilon
$$

de modo que $\mu$ é também uma medida interiormente regular.

Portanto, $\mu$ é uma medida regular, e isto encerra a demonstração.

Imediatamente segue a medida de Lebesgue é uma medida de Radon. Graças à Proposição 2.23, ao Teorema 2.24 e ao Teorema 2.30, sabemos que a $m$-medida de Hausdorff é uma medida de Borel finita nos compactos de $\mathbb{R}^{m}$, logo é uma medida de Radon.

Mais do que isso, ambas são medidas de Haar em $\mathbb{R}^{m}$ e, como veremos, $\mathbb{R}^{m}$ admite uma única medida de Haar. E unicidade, neste caso, quer dizer que duas medidas de Haar são iguais, a menos de um ajuste por uma constante positiva. 
Definição D.6. Uma medida de Haar em $\mathbb{R}^{m}$ é uma medida de Radon em $\mathbb{R}^{m}$ invariante não nula.

Corolário D.7. A medida de Lebesbegue em $\mathbb{R}^{m}$ e a m-medida de Hausdorff em $\mathbb{R}^{m}$ são medidas de Haar.

Para continuarmos, precisamos definir a norma uniforme de uma função $f \in C_{\mathrm{c}}\left(\mathbb{R}^{m}\right)$, denotada por $\|f\|_{u}$,

$$
\|f\|_{u}:=\sup _{x \in \mathbb{R}^{m}}|f(x)|
$$

e também

$$
C_{\mathrm{c}}^{+}\left(\mathbb{R}^{m}\right):=\left\{f \in C_{\mathrm{c}}\left(\mathbb{R}^{m}\right): f \geqslant 0 \text { e }\|f\|_{u}>0\right\}
$$

Proposição D.8. Seja $\mu$ uma medida de Haar em $\mathbb{R}^{m}$.

Se $U \subset \mathbb{R}^{m}$ é um aberto não vazio e $f \in C_{\mathrm{c}}^{+}\left(\mathbb{R}^{m}\right)$, então $\mu(U)>0$ e

$$
\int f d \mu>0
$$

\section{Demonstração:}

Uma vez que $\mu$ é regular não nula, existe um compacto $K \subset \mathbb{R}^{m} \operatorname{com} \mu(K)>0$. Dado $U \subset \mathbb{R}^{m}$ aberto não vazio, sabemos que $K$ pode ser coberto por uma quantidade finita de conjuntos da forma $x+U$, com $x \in K$, e então $\mu(U)>0$.

Agora, defina $U=\left\{x \in \mathbb{R}^{m}: f(x)>\|f\|_{u} / 2\right\}$, daí

$$
\int f d \mu \geqslant \frac{\|f\|_{u}}{2} \mu(U)>0
$$

Teorema D.9. Se $\mu$ e $\nu$ são medidas de Haar em $\mathbb{R}^{m}$, então existe uma constante $c>0$ tal que $\mu=c \nu$.

\section{Demonstração:}

Fixada $g \in C_{\mathrm{c}}^{+}\left(\mathbb{R}^{m}\right)$ qualquer, defina $h \in C_{\mathrm{c}}^{+}\left(\mathbb{R}^{m}\right)$ por $h(x)=g(x)+g(-x)$, de modo que $h(x)=h(-x)$. 
Dada $f \in C_{\mathrm{c}}\left(\mathbb{R}^{m}\right)$, recorremos ao Teorema de Fubini (Teorema 1.22) para obter

$$
\begin{aligned}
\int h d \nu \int f d \mu & =\iint h(y) f(x) d \mu(x) d \nu(y) \\
& =\iint h(y) f(x+y) d \mu(x) d \nu(y) \\
& =\iint h(y) f(x+y) d \nu(y) d \mu(x) \\
& =\iint h(-x+y) f(y) d \nu(y) d \mu(x) \\
& =\iint h(-y+x) f(y) d \nu(y) d \mu(x) \\
& =\iint h(-y+x) f(y) d \mu(x) d \nu(y) \\
& =\iint h(x) f(y) d \mu(x) d \nu(y) \\
& =\int h d \mu \int f d \nu .
\end{aligned}
$$

Note que o Teorema de Fubini é aplicável neste caso, pois as funções envolvidas têm suporte compacto, o qual tem medida finita. Pela Proposição D.8, sabemos que $\int h d \nu \neq$ 0 , de modo que pondo $c=\frac{\int h d \mu}{\int h d \nu}$, obtemos

$$
\int f d \mu=c \int f d \nu
$$

Agora, considere $T: C_{\mathrm{c}}\left(\mathbb{R}^{m}\right) \rightarrow \mathbb{R}$ e $S: C_{\mathrm{c}}\left(\mathbb{R}^{m}\right) \rightarrow \mathbb{R}$ no Teorema D.3 como sendo

$$
T(f)=\int f d \mu
$$

e

$$
S(f)=\int f d \nu .
$$


Dado um aberto $U \subset \mathbb{R}^{m}$,

$$
\begin{aligned}
\mu(U) & =\sup \left\{T(f): f \in C_{\mathrm{c}}\left(\mathbb{R}^{m}\right) \operatorname{com} 0 \leqslant f \leqslant 1 \mathrm{e} \operatorname{supp}(f) \subset U\right\} \\
& =\sup \left\{c \int f d \nu: f \in C_{\mathrm{c}}\left(\mathbb{R}^{m}\right) \operatorname{com} 0 \leqslant f \leqslant 1 \mathrm{e} \operatorname{supp}(f) \subset U\right\} \\
& =c \sup \left\{\int f d \nu: f \in C_{\mathrm{c}}\left(\mathbb{R}^{m}\right) \operatorname{com} 0 \leqslant f \leqslant 1 \operatorname{esupp}(f) \subset U\right\} \\
& =c \sup \left\{S(f): f \in C_{\mathrm{c}}\left(\mathbb{R}^{m}\right) \operatorname{com} 0 \leqslant f \leqslant 1 \operatorname{esupp}(f) \subset U\right\} \\
& =c \nu(U) .
\end{aligned}
$$

Novamente, usando que $\mu$ e $\nu$ são exteriormente regulares, concluímos que

$$
\mu=c \nu
$$

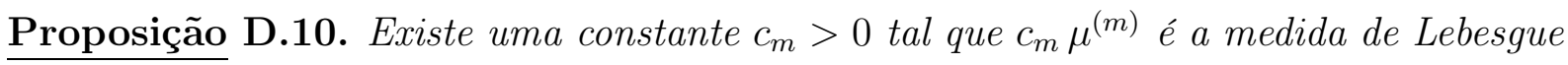
em $\mathbb{R}^{m}$.

\section{Demonstração:}

Sabidamente, $\mu^{(m)}$ e $\mathcal{L}^{m}$ são medidas de Haar (Corolário D.7), e então pelo Teorema D.9 concluímos que existe tal constante $c_{m}$ de forma que

$$
\mathcal{L}^{m}(E)=c_{m} \mu^{(m)}(E)
$$

para todo boreliano $E \subset \mathbb{R}^{m}$.

Mais pode ser dito sobre a constante $c_{m}$ : ela é de fato o volume de uma bola de diâmetro unitário, i.e.,

$$
c_{m}=\frac{\sqrt{\pi^{m}}}{2^{m} \Gamma\left(\frac{m}{2}+1\right)} .
$$

E isto advém de um resultado bem conhecido de geometria: a Desigualdade Isodiamétrica (Diestel [10], Theorem 1.8), segundo a qual

$$
\frac{\mathcal{L}^{m}(E)}{\mathcal{L}^{m}\left(B_{\mathbb{R}^{m}}(0, r / 2)\right)} \leqslant \frac{\operatorname{diam}(E)^{m}}{\operatorname{diam}\left(B_{\mathbb{R}^{m}}(0, r / 2)\right)^{m}}
$$


para todo boreliano $E \subset \mathbb{R}^{m}$. Confira a Proposição 2.29. Acontece que ela é uma consequência da Desigualdade de Brunn-Minkowski (Diestel [10], Theorem 1.7),

$$
\sqrt[m]{\mathcal{L}^{m}\left(E_{1}+E_{2}\right)} \geqslant \sqrt[m]{\mathcal{L}^{m}\left(E_{1}\right)}+\sqrt[m]{\mathcal{L}^{m}\left(E_{2}\right)}
$$

e de um fato muito intuitivo:

\section{Teorema D.11. (Eggleston [14], Remark on pg. 106)}

Dentre todos os conjuntos de $\mathbb{R}^{m}$ de diâmetro unitário, a bola é o de volume máximo.

Em Falconer [17], prova-se o Teorema D.10 sucintamente invocando a Proposição D.11 e o Teorema da Cobertura de Vitali (Falconer [17], Theorem 1.10); com isso, evita-se o tratamento por medidas de Haar e, de imediato, explica o porquê de não o seguirmos. 


\section{Índice Remissivo}

$\alpha$-limite de uma solução global, 34

$\omega$-limite

de uma solução global, 34

definição, 30

Órbita

definição, 23

periódica, 24

Afinamento (shrinking), 58

Atrator

exponencial, 128

global, 28

local, 43

Base de Auerbach, 138

Conjunto

de primeira categoria, $\mathbf{1 6}$

de segunda categoria, 16

em posição geral em $\mathbb{R}^{m}, 102$

estável local, 45

geometricamente independente, 102

instável, 41

instável local, 45

invariante, 23

invariante fonte, 45 invariante isolado, 38

invariante maximal, 38

invariante sela, 45

invariante sumidouro, 45

que absorve, 34

que atrai, 27

repulsor (complementar), 43

separado, 160

Dimensão

de Hausdorff, 72

fractal, 87

grande indutiva, 167

pequena indutiva, 95, 166

topológica, 49, 55, 166

Distância

de Banach-Mazur, 139

simétrica de Hausdorff, 27

Engrossamento (swelling), $\mathbf{5 6}$

Espaço

compacto, 12

de Baire, 16

dual, 18

paracompacto, 13 
separável, 13

Estrutura homoclínica, 39

Família

essencial, 51

localmente finita, 12

Família finita disjunta de invariantes isolados, $\mathbf{3 8}$

Função

C-Lipschitz, 82

bi-Lipschitz, 82

de Lyapunov, 39

de Urysohn, 14

Integral superior, 175

Lema de Urysohn, 13

Medida

de Haar, 195

de Hausdorff, 70

de Radon, 185

exterior métrica, 74

exterior produto, 175

produto, 175

regular, 185

Número de Lebesgue, 18

Ordem (de uma cobertura), 49

Par atrator-repulsor, 43

Partição

da unidade, 14

entre dois conjuntos fechados

disjuntos, 51

Ponto

de equilíbrio, $\mathbf{2 4}$

fixo, 15
Refinamento, 56

Semi-órbita positiva, 29

Semidistância de Hausdorff, 26

Semigrupo, 22

(limitado) dissipativo, 34

assintoticamente compacto, $\mathbf{3 1}$

eventualmente compacto, 36

eventualmente limitado, 36

gradiente, 39

limitado, 36

ponto dissipativo, 36

Solução global

definição, 23

periódica, 24

Taxa de atração, 129

Teorema

da Categoria de Baire, 17

da Convergência Monótona, 20, 182

da existência de partições da

unidade, 14

da Imersão, 104

da Imersão para Espaços Métricos

Separáveis, 108

da Representação de Riesz para

medidas de Radon, 187

de Banach-Alaoglu, 19

de Carathéodory, 20

de Fubini-Tonelli, 21

de Hahn-Banach, 19

de Mañé, 114

de Weierstrass, 16

do Ponto Fixo de Brouwer, 16

Topologia

fraca, 18

fraca*, 18 


\section{Referências Bibliográficas}

[1] Aragão-Costa, E.R.; Caraballo, T.; Carvalho, A.N.; Langa, J.A. Stability of gradient semigroups under perturbations, Nonlinearity 24 2099-2117 2012.

[2] Bartle, R.G. The Elements of Integration and Lebesgue Measure. New York: John Wiley \& Sons, 1995.

[3] Bortolan, M.C; Caraballo, T.; Carvalho, A.N. and Langa, J.A., An estimate on the fractal dimension of attractors of gradient-like dynamical systems. Nonlinear Analysis: Theory, Methods \& Applications, Vol. 75 (2012) 5702-5722.

[4] Brezis, H. Functional Analysis, Sobolov Spaces and Partial Differential Equations. New York: Springer, 2011.

[5] Cabrelli, C.; Molter, U.; Paulauskas, V.; Shonkwiler, R. Hausdorff measure of pCantor sets. Real Analysis Exchange. v.30, n.2 (2004), p.413 - 434.

[6] Carvalho, A.N., Langa, J. A. and Robinson, J. C., Attractors for infinite dimensional non-autonomous dynamical systems, Applied Mathematical Sciences 182 SpringerVerlag 2012.

[7] Carvalho, A.N., Langa, J. A., and Robinson, J. C. Finite-dimensional global attractors in Banach spaces. Journal of Differential Equations, 249 (12) 3099-3109 (2010).

[8] Carvalho, A.N. Sistemas dinâmicos não lineares. São Carlos: ICMC-USP 2012. http://www.icmc.usp.br/ andcarva/SDNL2012.pdf

[9] Carvalho, A.N.; Sonner, S. Pullback Exponential Attractors for evolution processes in Banach Spaces: properties and applications. Communications on Pure and Applied Analysis 3 (2014), 1141-1165. 
[10] Diestel, J.; Spalsbury, A. The Joys of Haar Measure. American Mathematical Society: Graduate Studies in Mathematics, vol.150, 2013.

[11] Edgar, G.A. Integral, Probability and Fractal Measures. New York: Springer-Verlag, 1998.

[12] Edgar, G.A. Measure, Topology and Fractal Geometry. 2. ed. New York: Springer, 2008.

[13] Efendiev, M.; Miranville, A.; Zelik, S. Exponential attractors and finite-dimensional reduction for nonautonomous dynamical systems. Proceedings of the Royal Society of Edinburgh, v. 135A, p. 703-730, 2005.

[14] Eggleston, H.G. Convexity. London: Cambridge University Press, 1958.

[15] Engelking, R. Dimension Theory. Amsterdam: North-Holland, 1978.

[16] Falconer, K.J. Fractal Geometry. Mathematical Foundations and Applications. 2. ed. St Andrews: John Wiley \& Sons, 2003.

[17] Falconer, K.J. The Geometry of Fractal Sets. New York: Cambridge University Press, 1985.

[18] Folland, G.B. Real Analysis: Modern Techniques and Their Applications. 2. ed. New York: John Wiley \& Sons, 1999.

[19] Hale, J.K. Asymptotic Behavior of Dissipative Systems, Mathematical Surveys and Monographs 25. American Mathematical Society, Providence, RI, 1988.

[20] Hurewicz, W.; Wallman, H. Dimension Theory. Princeton University Press, 1941.

[21] Mill, J.V. Infinite-Dimensional Topology. Prerequisites and Introduction. New York: Eslsevier, 1989.

[22] Lima, E.L. Espaços Métricos. Rio de Janeiro: Projeto Euclides, IMPA, 1977.

[23] J. Mallet-Paret, Negatively invariant sets of compact maps and an extension of a theorem of Cartwright, J. Differential Equations, 22, (1976) $331 ? 348$.

[24] Mañé, R. On the dimension of the compact invariant sets of certain non-linear maps. Lecture Notes in Mathematics 898, Springer-Verlag, New York, pp. 230-242, 1981. 
[25] Munkres, J.R. Topology. 2. ed. Bergen County: Prentice Hall, 2000.

[26] Pesin, Y.B. Dimension Theory in Dynamical Systems: Contemporary Views and Applications. Chicago: The University of Chicago Press, 1997.

[27] Robinson, J.C. Dimensions, Embeddings, and Attractors. Cambridge Tracts in Mathematics 186, 2010.

[28] Temam, R. Infinite dimensional dynamical systems in mechanics and physics. Applied Mathematical Sciences 68, Springer-Verlag, 1997. 